\title{
II EMPIRISChe Resultate
}

\section{SoZIODEMOGRAPHISCHE GrUNDSTRUKTUR}

Abb. 1.1: Altersstruktur (gesamt und nach Herkunft)

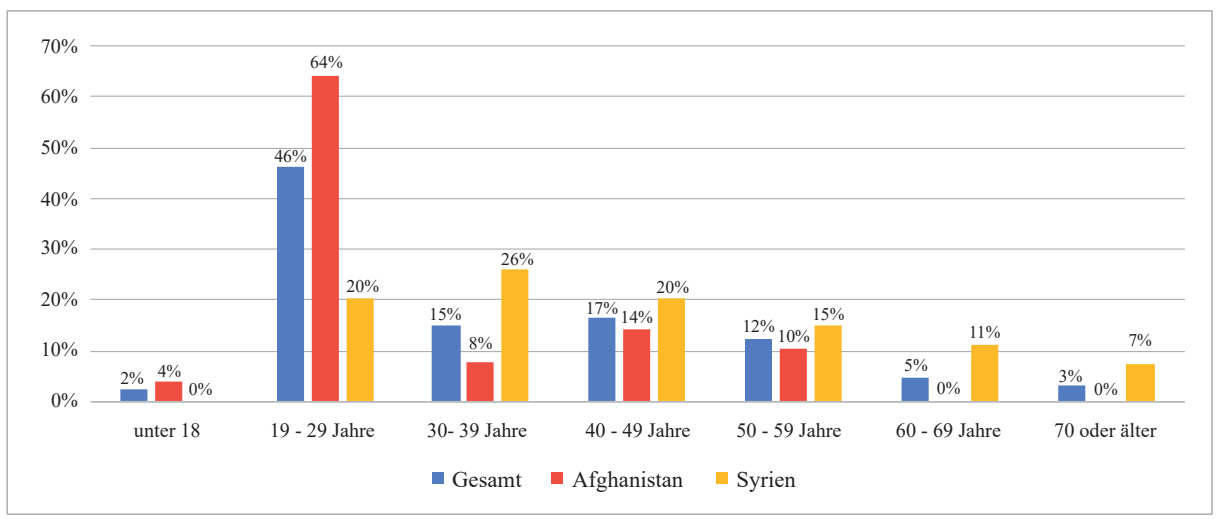

Quelle: eigene Erhebung 2020.

Der Gesamtrücklauf der Online-Befragung umfasste 132 beantwortete Fragebögen. Von diesen entfielen 54 in arabischer Sprache befüllte auf die syrischen Communities, von denen 10 in analoger Form komplettiert und nachträglich eingegeben wurden. In den afghanischen Communities konnten 78 Fragebögen gesammelt werden. Davon wurden 57 in deutscher Sprache und 21 auf Farsi-Dari befüllt. Alle Fragebogen des afghanischen Subsamples wurden im Rahmen der Online-Version beantwortet.

Im Sample dominieren die männlichen Befragten mit 56\%. Bei den Afghan/inn/en haben 40 Männer und 38 Frauen den Online-Fragebogen ausgefüllt (vgl. Abb. 1.2). Die Geschlechterverteilung spiegelt also im Fall der Afghan/inn/en mit 51\% männlichen Interviewees die für die meisten Fluchtmigrationsbewegungen typische anteilsmäßige Dominanz der Männer nicht wider. Unter den Syrer/inne/n ist das Geschlechterverhältnis mit 34 zu 20 erheblich unausgewogener, d.h., der Männerüberhang tritt mit 63\% deutlich zutage. Der hohe Frauenanteil in der Subgruppe aus Afghanistan ist auf eine Oversampling-Strategie zurückzuführen. Da das Forscherteam vor allem auch Aufschluss über die genderspezifischen Herausforderungen der COVID-19-Pandemie 
für geflüchtete Frauen gewinnen wollte, wurden die partizipierenden Vereine gebeten, besonders afghanische Frauen für die Teilnahme an der Befragung zu motivieren.

Abb. 1.2: Geschlechterverteilung (gesamt und nach Herkunft)

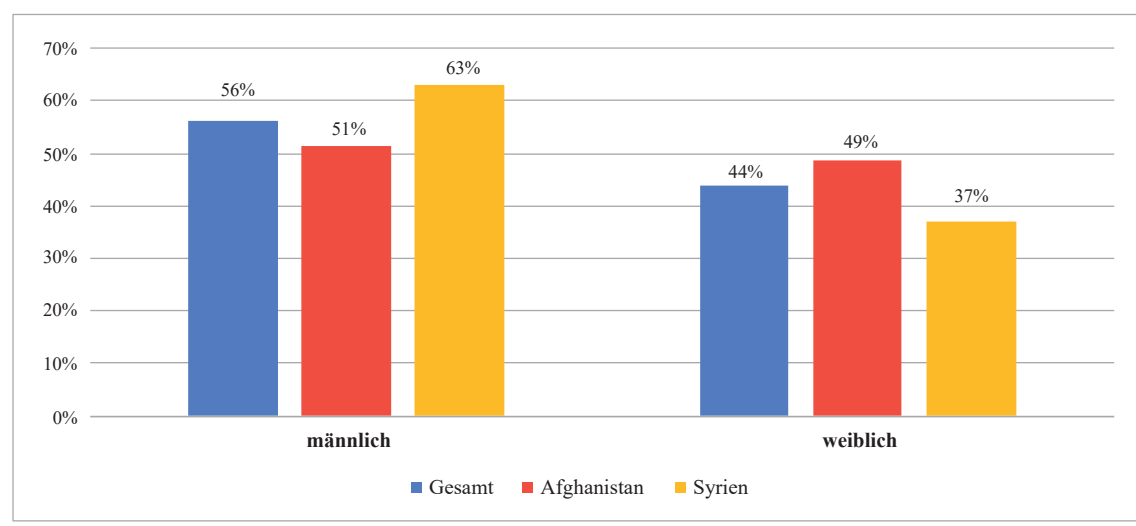

Quelle: eigene Erhebung 2020.

Die Altersstruktur des Samples (vgl. Abb. 1.1) spiegelt jene in der Grundgesamtheit der Personen aus den beiden Herkunftsstaaten Afghanistan und Syrien weitgehend wider, denn in beiden Gruppen dominieren die jüngeren Alterskohorten zwischen 19 und 29 Jahren (46\% aller Befragten). Bei den Respondent/inn/en aus Afghanistan sind sogar 64\% der Respondent/inn/en dieser Alterskategorie zuzuordnen (vgl. KOHLBACHER et al. 2020; BAUER et al. 2017). Geflüchtete aus Syrien befinden sich etwas öfter auch bereits in einem höheren Alter. Darüber hinaus verteilen sich die Afghan/inn/en auch bereits

Abb. 1.3: Familienstand (gesamt und nach Herkunft)

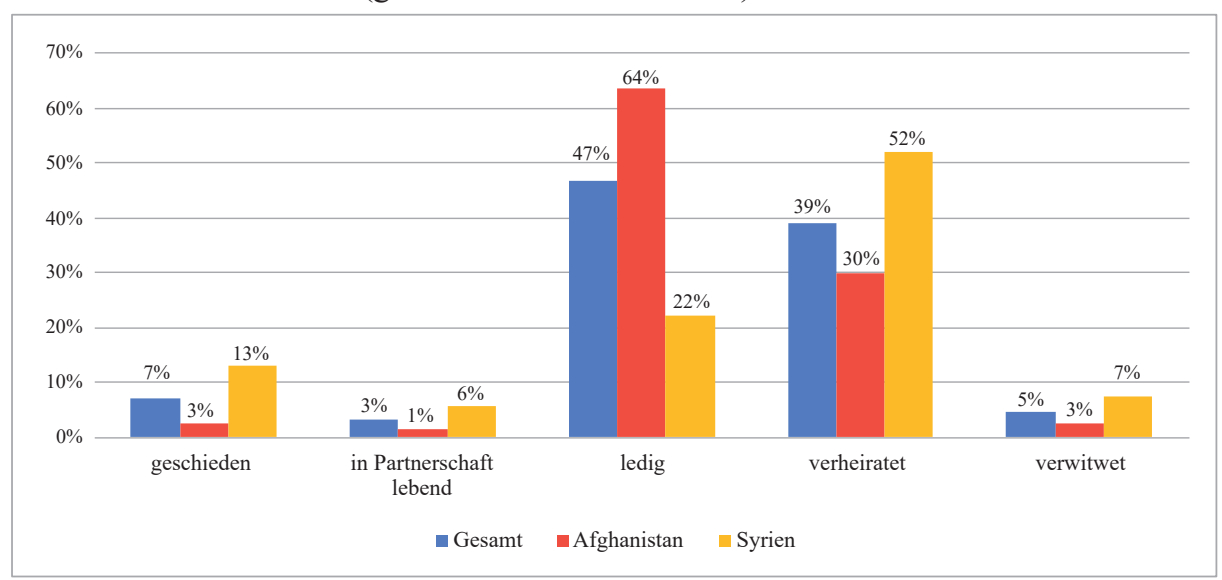

Quelle: eigene Erhebung 2020. 
in einem höheren Alter. Darüber hinaus verteilen sich die Afghan/inn/en auch auf die Altersgruppen von 30 bis 39 (8\%), 40 bis 49 (14\%) und 50 bis 59 Jahren (10\%). Allerdings ist kein einziger afghanischer Befragter älter als 59 Jahre. Im Vergleich dazu verteilen sich die interviewten Syrer/innen gleichmäßiger auf die gesamte Bandbreite an Altersgruppen. So ist ihr Anteil unter den jungen Befragten (bis 29 Jahre) zwar beträchtlich geringer (20\%) als unter den Afghan/inn/en, dafür finden sich darunter zahlreiche Personen im mittleren Erwerbsalter (also zwischen 30 und 59 Jahren mit 26\%) und auch Menschen im Pensionsalter ( $\mathrm{ab} 60$ und sogar 70 Jahre und älter mit insgesamt 18\%).

Die Abb. 1.3 zeigt, dass hinsichtlich des Familienstandes das Schwergewicht im Sample bei ledigen Personen (47\%) liegt. In erster Linie in der afghanischen Herkunftsgruppe bilden diese das Gros der Befragten (64\%), was auch mit deren junger Altersstruktur zusammenhängt. Deutlich weniger Afghan/inn/en sind bereits verheiratet (30\%). In der Subgruppe aus Syrien findet sich ein beträchtlich höherer Anteil von verheirateten Befragten (52\%) und erheblich weniger ledige Personen (22\%), dafür haben aber mehr geschiedene (13\%), verwitwete (7\%) und in Partnerschaften lebende (6\%) Respondent/inn/en an der Online-Befragung teilgenommen.

Abb. 1.4: Staatsbürgerschaft (nach Herkunft)

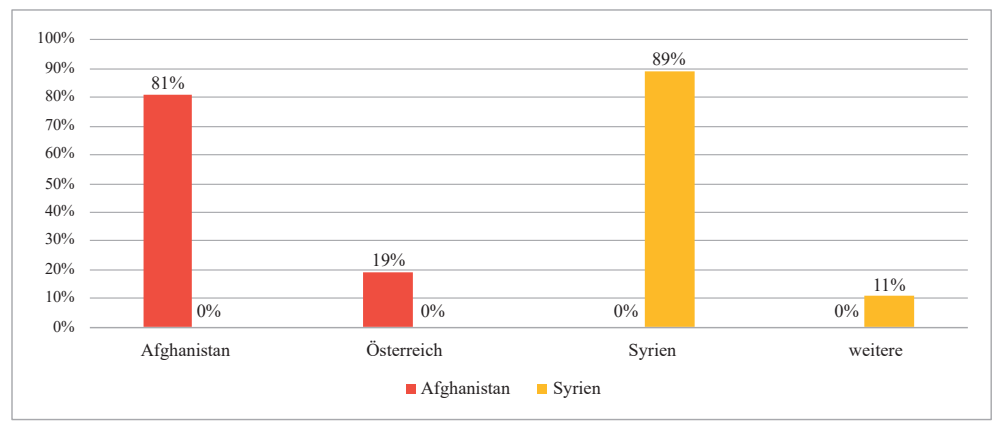

Quelle: eigene Erhebung 2020.

Wie aus Abb. 1.4 hervorgeht, waren 63 Befragte aus Afghanistan (81\%) zum Befragungszeitpunkt noch ehemalige afghanische Staatsbürger/innen, d.h. seit Stellung des Asylantrags eigentlich staatenlos, und 15 (19\%) bereits eingebürgert. Letzteres erklärt sich daraus, dass an der Onlinebefragung auch ein größerer Anteil von bereits länger in Österreich aufhältigen Personen mit afghanischem Migrationshintergrund partizipierten. Anders die Verteilung in der Subgruppe aus Syrien: In dieser hatten 48 $(89 \%)$ noch keine neue Staatsbürgerschaft bzw. die syrische Staatsbürgerschaft aufgegeben, sechs Befragte (11\%) waren Bürger/innen anderer Staaten, aber kein/e/r der Syrer/innen war österreichische/r Staatsbürger/in.

In Bezug auf das Jahr der Zuwanderung und somit die Aufenthaltsdauer in Österreich manifestieren sich deutliche Divergenzen zwischen den beiden Gruppen (vgl. Abb. 1.5). Bei beiden gelangte zwar die Mehrzahl (insgesamt 37\%, davon $50 \%$ bei den 
Abb. 1.5: Ankunftsjahr (gesamt und nach Herkunft)

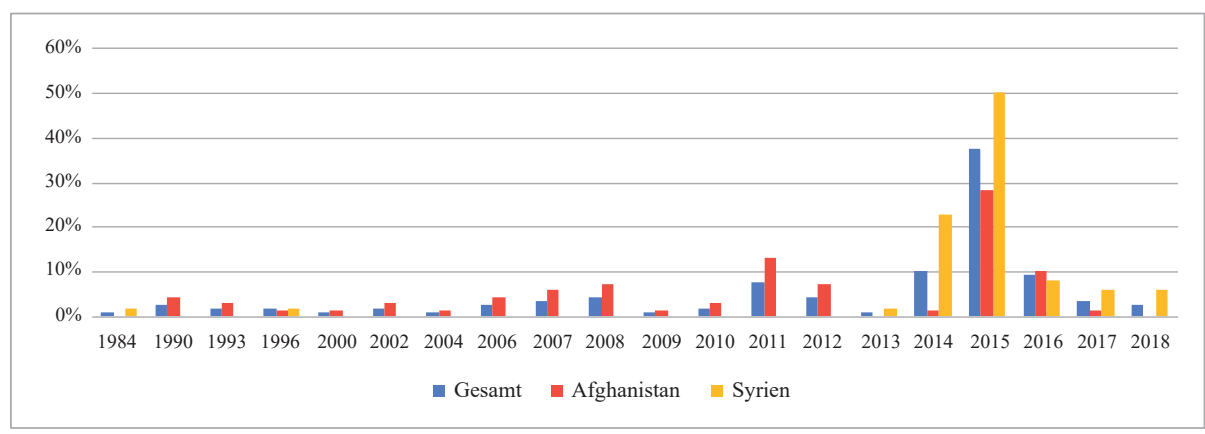

Quelle: eigene Erhebung 2020.

Syrer/inne/n und 28\% bei den Afghan/inn/en) im Jahr 2015 nach Österreich. Bei den Syrer/inne/n floh ein erheblicher Anteil (23\%) auch bereits im Jahr unmittelbar davor und in summa 20\% in den Jahren 2016 (8\%), 2017 (6\%) und $2018(6 \%)$. Unter den Afghan/inn/en finden sich sieben Personen, die 2016 nach Österreich migrierten, eine größere Zahl von ihnen (29) ist aber bereits erheblich früher, nämlich im Zeitraum von 2006 bis 2012, in Österreich eingetroffen. Diese Unterschiede lassen sich zum Teil auf die im Kontext des partizipativen Forschungsansatzes in die Befragung involvierten Vereine und deren Mitgliederstruktur zurückführen. Vor allem im afghanischen Kulturverein AKIS-EU sind viele Mitglieder bereits längere Zeit in Österreich ansässig, einige inzwischen auch eingebürgert.

Abb. 1.6: Berufsgruppen vor der Coronakrise und zum Befragungszeitpunkt (gesamt)

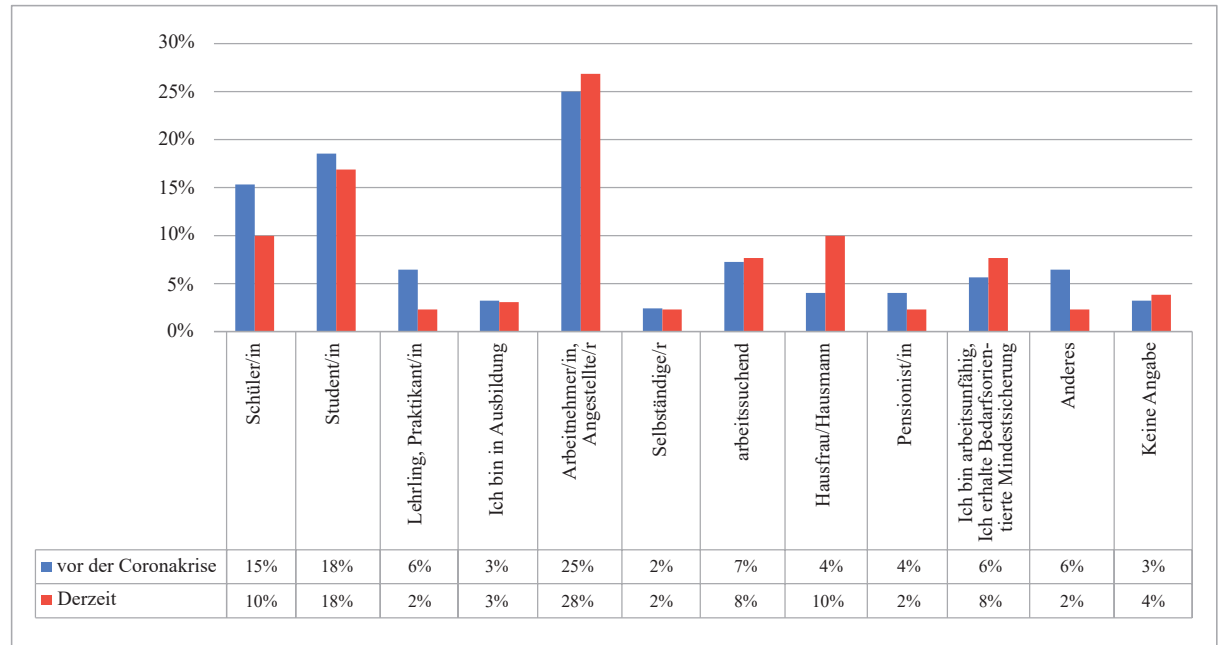

Quelle: eigene Erhebung 2020. 
Hinsichtlich der beruflichen Tätigkeit dominieren im gesamten Sample die Personen in Angestelltenpositionen, weitere größere Gruppen bilden aufgrund der jungen Altersstruktur die Studierenden sowie auch die Schüler/innen (vgl. Abb. 1.6). Die Zahl und der Anteil der Arbeitsuchenden sind relativ niedrig. Sehr klein ist unter den Angehörigen der rezenten Fluchtmigrationsbewegungen aus Afghanistan und Syrien die Präsenz selbständig Erwerbstätiger. Arbeitssuchende und Bezieher/innen der bedarfsorientierten Mindestsicherung sowie im Haushalt Tätige bilden ebenfalls nennenswerte Gruppen.

Die Veränderungen hinsichtlich der beruflichen Tätigkeiten vor der Coronakrise und zum Zeitpunkt der Erhebung treten in den Berufsgruppen sehr unterschiedlich zutage. So war der Anteil der Arbeitnehmer/innen bzw. Angestellten vor der Coronakrise (25\%) geringer als zum Erhebungszeitpunkt (28\%). Höher waren vor Corona die Zahlen der Schüler/innen (Rückgang von 15\% auf 10\%) und in einem Lehrverhältnis (von 6\% auf 2\%) stehenden Personen, während der Anteil in Ausbildung stabil geblieben ist. Der Rückgang in der Kategorie „Lehrlinge“ ist darauf zurückzuführen, dass diese Gruppe auch Praktikant/inn/en inkludiert und deren Praktika ausgelaufen sind und nicht verlängert wurden. Etwas verringert hat sich auch der Anteil der Pensionist/inn/en, was aber - nach entsprechender Prüfung - auf eine Fehlinterpretation der Befragten zurückzuführen war. Absolut und relativ am stärksten zugenommen hat die Kategorie der im Haushalt tätigen Befragten (von 4\% auf 10\%), aber auch bei arbeitsunfähigen Personen zeigt sich im Gefolge der Krise ein Anstieg von 6\% auf 8\%. Gar nicht verändert (jeweils $2 \%$ ) hat sich infolge der Krise der geringe Anteil der unternehmerisch tätigen Personen im Sample. Wenig überraschend ist, dass der Anteil der arbeitssuchenden Respondent/ inn/en von $7 \%$ auf $8 \%$ angestiegen ist.

Abb. 1.6.1: Berufsgruppen (Herkunft Afghanistan)

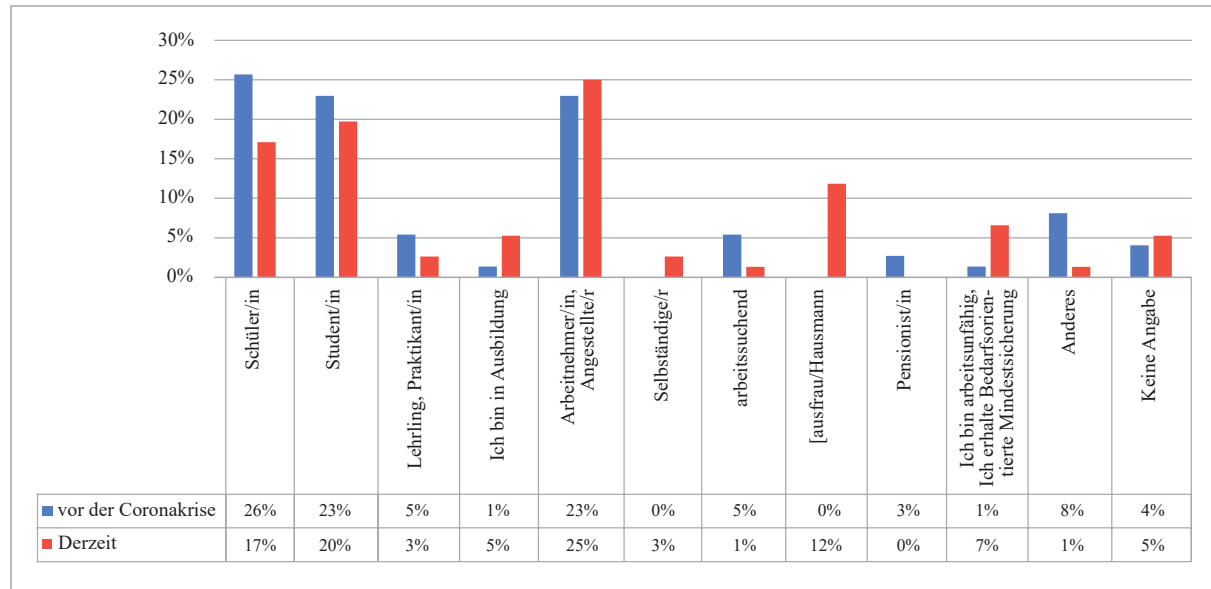

Quelle: eigene Erhebung 2020. 
Die Struktur nach Berufsgruppen (vgl. Abb. 1.6.1 Afghanistan) zeigt im Vergleich der Zahlen vor der Coronakrise und zum Zeitpunkt der Erhebung in den afghanischen Communities Zuwächse bei den in Ausbildungsverhältnissen stehenden Personen (von $1 \%$ auf 5\%) sowie in der Gruppe der Arbeitsunfähigen (von 1\% auf 7\%). Reduziert haben sich die Kategorien der Schüler/innen (von 26\% auf 17\%), der Studierenden (von 23 auf 20\%) und der Lehrlinge/Praktikant/inn/en (5\% auf 3\%). Der Anteil der Arbeitnehmer/innen ist um 2\% gestiegen, bei 3\% lag der Zuwachs bei den Selbständigen, noch stärker aber war der Anstieg bei den im Haushalt Tätigen (12\%).

Abb. 1.6.2: Berufsgruppen (Herkunft Syrien)

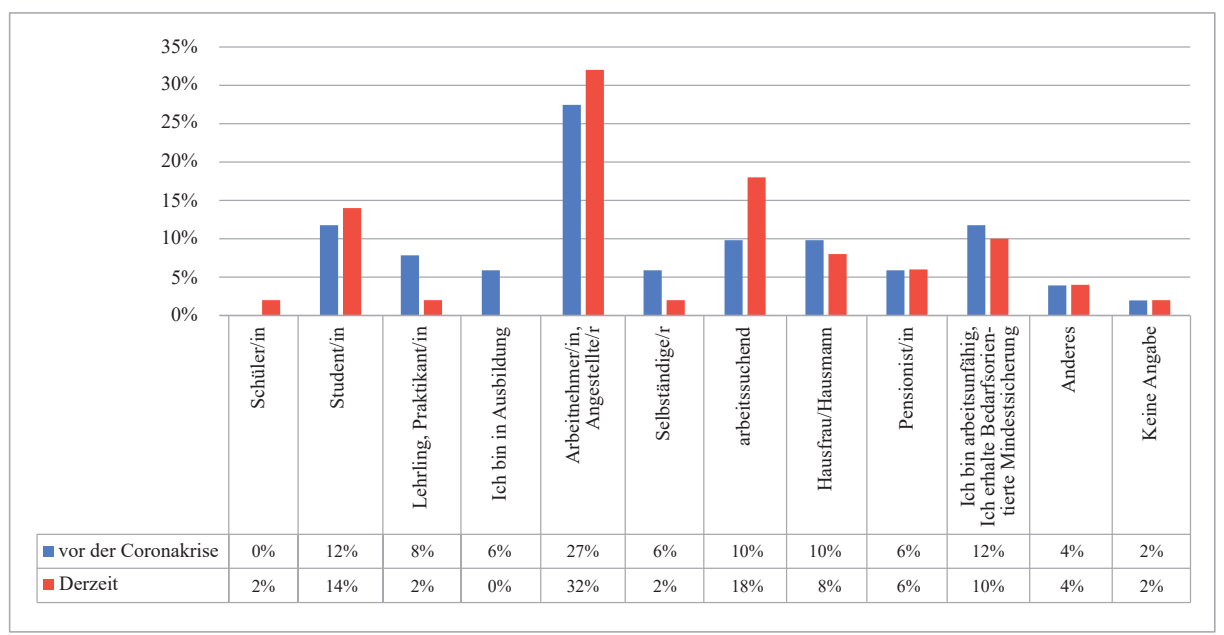

Quelle: eigene Erhebung 2020.

In der Herkunftsgruppe Syrien (vgl. Abb. 1.6.2 Syrien) sind im Vergleich des Status vor der Coronakrise und zum Erhebungszeitpunkt vor allem die Anteile der Arbeitsuchenden (von 10\% auf 18\%) sowie in geringerem Ausmaß jene der Studierenden (von $12 \%$ auf 14\%) und der Arbeitnehmer/innen (von 27\% auf 32\%) angestiegen. Leicht reduziert hat sich der Anteil der im Haushalt Tätigen (von 10\% auf 8\%) sowie der Personen, die arbeitsunfähig waren (von 12\% auf 10\%). Stabil geblieben ist der Anteil der Pensionist/inn/en. Extrem ausgeprägt war der Rückgang bei den Lehrlingen bzw. Praktikant/inn/en (von 8\% auf 2\%), bei den in Ausbildung stehenden Befragten (von 6\% auf 0 ) sowie bei den Selbständigen (von 6\% auf $2 \%$ ).

Auch hinsichtlich des Berufes vor und nach der Coronakrise zeigen sich interessante Unterschiede auf Basis der Analyse nach der Gendervariable (vgl. Tab. 1). Zwar war der Angestelltenanteil bei beiden Geschlechtern nahezu gleich hoch, Arbeitnehmer/ innen waren aber mehr als 26\% der Männer gegenüber rund $17 \%$ der weiblichen Befragten. Die bedarfsorientierte Mindestsicherung erhielten wesentlich mehr Frauen 
$(8,6 \%)$ als Männer (6,9\%). Die Anteile der in Ausbildung befindlichen Personen bzw. der Lehrlinge variierten ebenfalls stark nach dem Geschlecht. Fast 7\% der Männer und nur 1,7\% der Frauen machten ein Praktikum oder eine Lehre, bei den Befragten in Ausbildung war der Unterschied geringer. Hingegen waren wesentlich mehr Frauen (fast 14\%) noch Schülerinnen (Männer zu 8,3\%) und Analoges galt für die Studierenden (17,2\% Frauen und 8,3\% Männer). Auch Studierende mit Teilzeitjobs waren viel häufiger Frauen (10,3\% gegenüber 2,8\% der Männer).

Tab. 1: Beruf vor der Coronakrise nach Geschlecht (gesamt)

\begin{tabular}{|l|r|r|r|}
\hline \multicolumn{1}{|c|}{ Beruf vor der Coronakrise } & männlich & weiblich & Gesamtergebnis \\
\hline Ungültige & $11,11 \%$ & $10,34 \%$ & $10,77 \%$ \\
\hline Anderes & $5,56 \%$ & $5,17 \%$ & $5,38 \%$ \\
\hline Arbeitnehmer/in, Angestellte/r & $26,39 \%$ & $17,24 \%$ & $22,31 \%$ \\
\hline arbeitssuchend & $6,94 \%$ & $1,72 \%$ & $4,62 \%$ \\
\hline Hausfrau/Hausmann & $2,78 \%$ & $6,90 \%$ & $4,62 \%$ \\
\hline $\begin{array}{l}\text { Ich bin arbeitsunfähig, Ich erhalte } \\
\text { Bedarfsorientierte Mindestsicherung }\end{array}$ & $6,94 \%$ & $8,62 \%$ & $7,69 \%$ \\
\hline Ich bin in Ausbildung & $6,94 \%$ & $5,17 \%$ & $6,15 \%$ \\
\hline Lehrling, Praktikant/in & $6,94 \%$ & $1,72 \%$ & $4,62 \%$ \\
\hline Pensionist/in & $2,78 \%$ & $1,72 \%$ & $2,31 \%$ \\
\hline Schüler/in & $8,33 \%$ & $13,79 \%$ & $10,77 \%$ \\
\hline Selbständige/r & $2,78 \%$ & $0,00 \%$ & $1,54 \%$ \\
\hline Student/in & $8,33 \%$ & $17,24 \%$ & $12,31 \%$ \\
\hline Student/in mit Teilzeitjob & $2,78 \%$ & $10,34 \%$ & $6,15 \%$ \\
\hline Gesamtergebnis & $\mathbf{1 0 0 , 0 0 \%}$ & $\mathbf{1 0 0 , 0 0 \%}$ & $\mathbf{1 0 0 , 0 0 \%}$ \\
\hline
\end{tabular}

Quelle: eigene Erhebung 2020.

Zum Befragungszeitpunkt hat sich die Struktur nach Berufsgruppen erheblich verändert (vgl. Tab. 2). Vor allem fällt der starke Zuwachs in der Kategorie ,anderes“ auf. Die Anteile der arbeitssuchenden Personen sind bei beiden Geschlechtern erheblich gestiegen. Dies ist darauf zurückzuführen, dass viele in der Krise ihren Job verloren haben. Stark reduziert hatten sich vor allem bei den Männern (Rückgang von rund 8\% auf 4\%) die Anteile der Studierenden. Bei den befragten Frauen fiel die Reduktion von $17 \%$ auf $15,5 \%$ insgesamt moderater aus. Unter den Männern gab es nach der ersten Krise 2020 auch viel weniger in Lehrausbildung/Praktikum stehende Personen (Reduktion von 6,9\% auf 1,4\%), ausgeprägt war auch die Reduktion der in einer Ausbildung stehenden Respondent/inn/en (von 6,9\% auf 2,8\%). Reduziert hat sich auch der Anteil der Schülerinnen, angestiegen ist dieser jedoch bei den Schülern. 
Tab. 2: Beruf zum Befragungszeitpunkt nach Geschlecht (gesamt)

\begin{tabular}{|l|r|r|r|}
\hline \multicolumn{1}{|c|}{ Beruf derzeit } & männlich & weiblich & Gesamtergebnis \\
\hline Ungültige & $22,22 \%$ & $27,59 \%$ & $24,62 \%$ \\
\hline Anderes & $2,78 \%$ & $1,72 \%$ & $2,31 \%$ \\
\hline Arbeitnehmer/in, Angestellte/r & $22,22 \%$ & $13,79 \%$ & $18,46 \%$ \\
\hline arbeitssuchend & $11,11 \%$ & $3,45 \%$ & $7,69 \%$ \\
\hline Hausfrau/Hausmann & $6,94 \%$ & $8,62 \%$ & $7,69 \%$ \\
\hline Hausfrau/Hausmann und arbeitsunfähig & $1,39 \%$ & $0,00 \%$ & $0,77 \%$ \\
\hline Ich bin arbeitsunfähig / Ich erhalte & & & \\
bedarfsorientierte Mindestsicherung & $4,17 \%$ & $6,90 \%$ & $5,38 \%$ \\
\hline Ich bin in Ausbildung & $2,78 \%$ & $0,00 \%$ & $1,54 \%$ \\
\hline Lehrling, Praktikant/in & $1,39 \%$ & $3,45 \%$ & $2,31 \%$ \\
\hline Pensionist/in & $2,78 \%$ & $1,72 \%$ & $2,31 \%$ \\
\hline Schüler/in & $11,11 \%$ & $10,34 \%$ & $10,77 \%$ \\
\hline Selbständige/r & $1,39 \%$ & $0,00 \%$ & $0,77 \%$ \\
\hline Student/in & $4,17 \%$ & $15,52 \%$ & $9,23 \%$ \\
\hline Student/in mit Teilzeitjob & $5,56 \%$ & $6,90 \%$ & $6,15 \%$ \\
\hline Gesamtergebnis & $\mathbf{1 0 0 , 0 0 \%}$ & $\mathbf{1 0 0 , 0 0 \%}$ & $\mathbf{1 0 0 , 0 0 \%}$ \\
\hline
\end{tabular}

Quelle: eigene Erhebung 2020.

Das Bildungsniveau im Sample war im Durchschnitt sehr hoch (vgl. Abb 1.7), was die Selektivität der Fluchtmigration aus den beiden Herkunftsländern widerspiegelte (vgl. BuBER-EnNSER et al. 2016). In Bezug auf das Bildungsniveau dominierten im Sample Befragte mit Abschlüssen höherer Schulen bzw. Matura (31\%). Sehr hoch war mit 25\% auch der Anteil der Universitäts- oder FH-Absolvent/inn/en, weitere $4 \%$ haben zwar eine Universität besucht, diese Ausbildung aber vor der Flucht nicht abgeschlossen. Hingegen hatten im unteren Segment des Bildungsspektrums 13\% nur die Grundschule abgeschlossen bzw. konnten 4\% keinerlei Schulbildung vorweisen und $2 \%$ bloß einen Pflichtschulabschluss. Lehrabschlüsse waren mit $10 \%$ vertreten und Diplome auf mittleren Bildungsniveaus mit 7\%.

Markante Unterschiede zeigen sich im gruppenübergreifenden Vergleich hinsichtlich des Bildungsniveaus. So ist der Anteil der Befragten mit Universitäts- oder Hochschulstudium bei den Syrer/inne/n mit 50\% beträchtlich höher als bei den Afghan/ inn/en (7\%). Letztere haben dafür öfter (38\% gegenüber 20\% bei den Syrer/inne/n) eine höhere Schule abgeschlossen. Insgesamt bewegt sich das Bildungslevel der Geflüchteten aus Afghanistan eher auf mittleren bzw. auch auf niedrigeren Levels. Unter den Letztgenannten befinden sich auch 4\% Personen ohne Schulbildung, 18\% mit Grund schule und 4\% mit Pflichtschulabschluss. Einige haben im Rahmen der Befragung die 
Abb. 1.7: Schulbildung (gesamt und nach Herkunft)

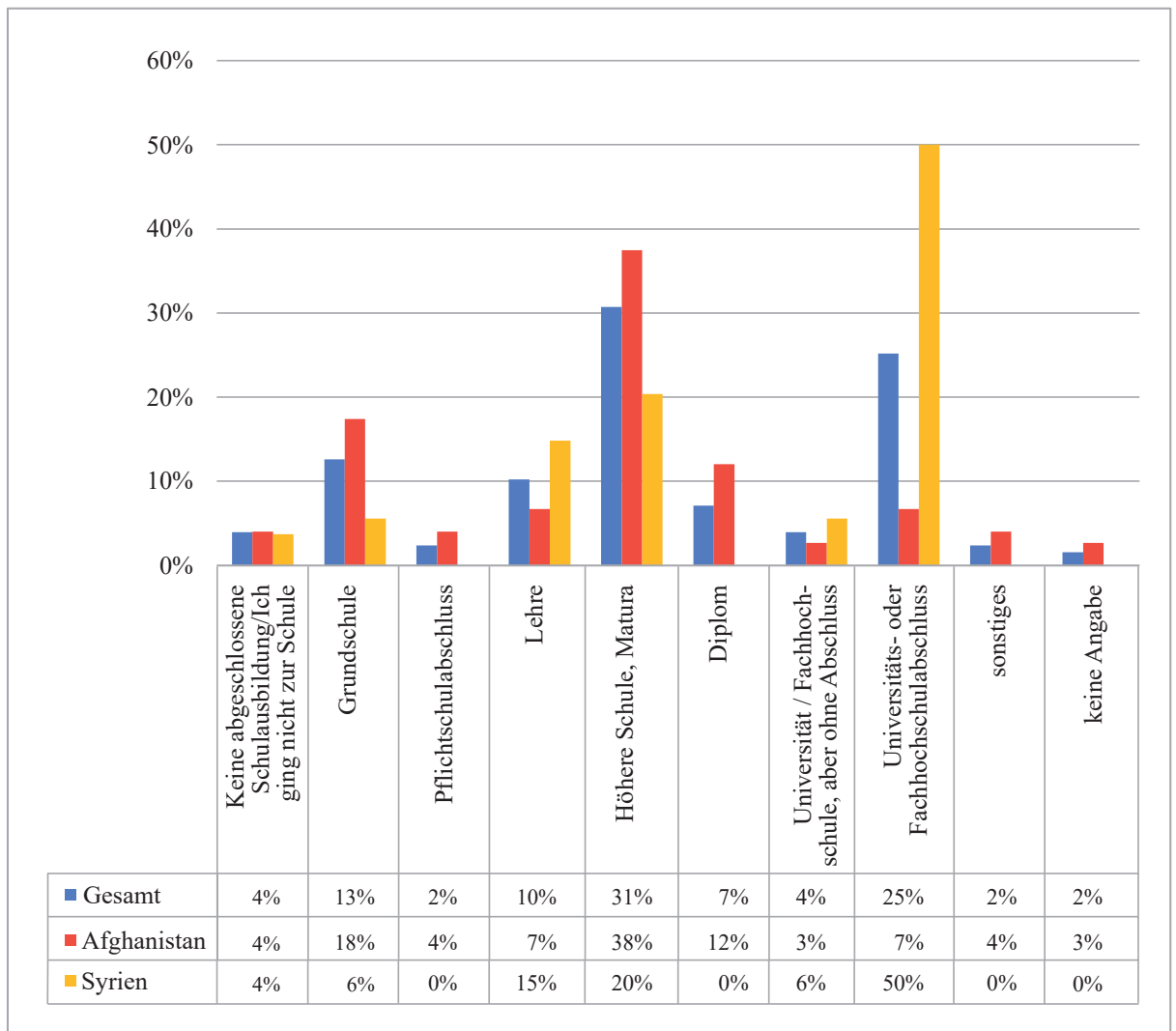

Quelle: eigene Erhebung 2020.

Koranschule als einzige Form der formalen Bildung angegeben. Der Bildungsstandard des Teilsamples aus Afghanistan ist somit signifikant geringer als jenes der Befragten aus Syrien. Damit entspricht die Struktur des Samples aber zu einem hohen Grad der Grundgesamtheit der Geflüchteten aus den beiden Herkunftsländern in Österreich (vgl. BUBER-ENNSER et al. 2016).

Unter den Syrer/inne/n liegt die Präsenz der Universitätsabsolvent/inn/en sogar bei $50 \%$ und somit beträchtlich höher als in den afghanischen Communities. Weitere $6 \%$ haben eine Hochschule zwar besucht, aber noch nicht abgeschlossen. Auch mittlere Bildungslevels sind bei den Personen aus Syrien stark vertreten, wenngleich etwas schwächer als bei den Afghan/inn/en. 20\% haben die Matura einer höheren Schule und $15 \%$ einen Lehrabschluss. Mit 10\% ist die Präsenz von Personen ohne oder nur mit abgeschlossener Grundschule erheblich geringer als bei den Afghan/inn/en. 
Tab. 3: Genderspezifische Unterschiede der Schulbildung (gesamt)

\begin{tabular}{|l|r|r|r|}
\hline & männlich & weiblich & Gesamtergebnis \\
\hline kein Bildungsabschluss & $9,72 \%$ & $5,45 \%$ & $7,87 \%$ \\
\hline primärer Bildungsabschluss & $25,00 \%$ & $25,45 \%$ & $25,20 \%$ \\
\hline sekundärer Bildungsabschluss & $33,33 \%$ & $43,64 \%$ & $37,80 \%$ \\
\hline tertiärer Bildungsabschluss & $31,94 \%$ & $25,45 \%$ & $29,13 \%$ \\
\hline Gesamtergebnis & $\mathbf{1 0 0 , 0 0 \%}$ & $\mathbf{1 0 0 , 0 0 \%}$ & $\mathbf{1 0 0 , 0 0 \%}$ \\
\hline
\end{tabular}

Quelle: eigene Erhebung 2020.

In Bezug auf die Bildungsabschlüsse ist der Männeranteil bei jenen, die keine Schule abgeschlossen haben mit fast $10 \%$ rund doppelt so hoch wie bei den Frauen im Sample (vgl. Tab. 3). Frauen verfügen in einem höheren Ausmaß über sekundäre Abschlüsse (43,6\% gegenüber 33,3\% bei den Männern), wohingegen die Männer eher eine Universitätsbildung (32\%) vorweisen können (Frauen nur zu einem Viertel).

Die Deutschkompetenz (vgl. Abb. 1.8.1) wurde auf Basis der Selbsteinschätzung abgefragt und wies keine weiteren Kontrollabfragen auf. In summa schätzten $32 \%$ der Respondent/inn/en ihre Deutschkenntnisse als sehr gut ein, weitere 27\% als gut. Nur 3\% bekundeten eine sehr schlechte und 13\% eine eher schlechte Deutschkompetenz.

Besonders die Afghan/inn/en haben ihre Kenntnisse zu 50\% als sehr gut, weitere $21 \%$ als gut eingeschätzt, wofür einerseits deren im Durchschnitt längere Aufenthaltsdauer in Österreich spricht und auch das Faktum, dass immerhin 57 (73\%) von ihnen

Abb. 1.8.1: Deutschkenntnisse (gesamt und nach Herkunft)

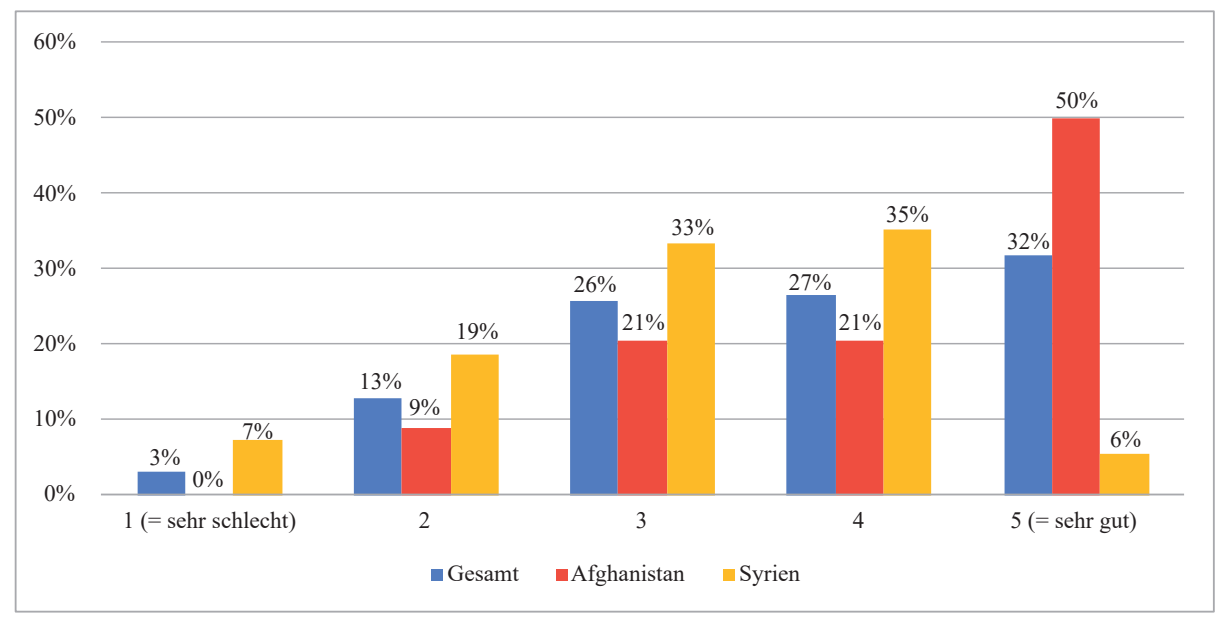

Quelle: eigene Erhebung 2020. 
im Rahmen der Online-Befragung die deutsche Variante des Fragebogens beantworteten. Vergleichsweise schwächer repräsentiert sind in der Kategorie der exzellenten Deutschkenntnisse (6\%) die Befragten aus Syrien. Diese geben häufiger eine eher gute (35\%) bis mittelgute (33\%) Deutschkompetenz an, in höherer Zahl aber auch geringe (19\%) bis sehr geringe (7\%) Kenntnisse der deutschen Sprache.Mit insgesamt 26\% sehr schlechter und eher schlechter Beherrschung der deutschen Sprache ist das Sprachlevel in der syrischen Subgruppe wesentlich schwächer als jenes der Afghan/inn/en.

Tab. 4: Geschlechtsspezifische Unterschiede in den Sprachkenntnissen (gesamt)

\begin{tabular}{|l|r|r|r|}
\hline & männlich & weiblich & $\begin{array}{c}\text { Gesamt- } \\
\text { ergebnis }\end{array}$ \\
\hline 1 (= sehr schlechte Deutschkenntnisse) & $1,17 \%$ & $0,43 \%$ & $0,82 \%$ \\
\hline 2 & $11,72 \%$ & $1,71 \%$ & $6,94 \%$ \\
\hline 3 & $19,92 \%$ & $21,79 \%$ & $20,82 \%$ \\
\hline 4 & $35,94 \%$ & $20,51 \%$ & $28,57 \%$ \\
\hline 5 (= sehr gute Deutschkenntnisse) & $31,25 \%$ & $55,56 \%$ & $42,86 \%$ \\
\hline Gesamtergebnis & $\mathbf{1 0 0 , 0 0 \%}$ & $\mathbf{1 0 0 , 0 0 \%}$ & $\mathbf{1 0 0 , 0 0 \%}$ \\
\hline
\end{tabular}

Darstellung als \% des Spaltengesamtergebnisses

Quelle: eigene Erhebung 2020.

Die Frauen im Sample weisen die besseren Deutschkenntnisse auf, wie Tab. 4 belegt. So ist der Anteil der Männer mit schlechter Deutschkompetenz (2) nahezu zehnmal so hoch wie bei den Frauen. Demgegenüber verfügen fast 56\% der weiblichen Befragten über exzellente Deutschkenntnisse gegenüber nur $31 \%$ bei den Männern.

Der Sprachkompetenz im Deutschen weitgehend entsprechend war die Einschätzung der Befragten bezüglich der eigenen Fähigkeit zur Rezeption von Nachrichtensendungen im TV bzw. Radio in deutscher Sprache (vgl. Abb. 1.8.2). Diese Kompetenz wurde von 37\% des gesamten Samples als sehr gut eingeschätzt, von 17\% als gut und von $26 \%$ als mittelmäßig. Das Gros (55\%) der Befragten aus Afghanistan beurteilte das Verstehen von Nachrichten in deutscher Sprache als sehr gut bzw. gut (17\%). Weitere $21 \%$ der Afghan/inn/en gaben eine mittlere Bewertung ab. Unter den Respondent/inn/ en aus Syrien wurden eher mittlere (35\%), eher schlechte $(20 \%)$ oder aber gute $(18 \%)$ Einschätzungen abgegeben. Sehr gut konnten nur 10\% der befragten Syrer/innen den deutschsprachigen TV-Nachrichten folgen, dafür waren 38\% nur sehr schlecht bzw. eher schlecht imstande deutsche Nachrichten verstehend zu rezipieren. 
Abb. 1.8.2: Verstehen von deutschsprachigen Rundfunknachrichten (gesamt und nach Herkunft)

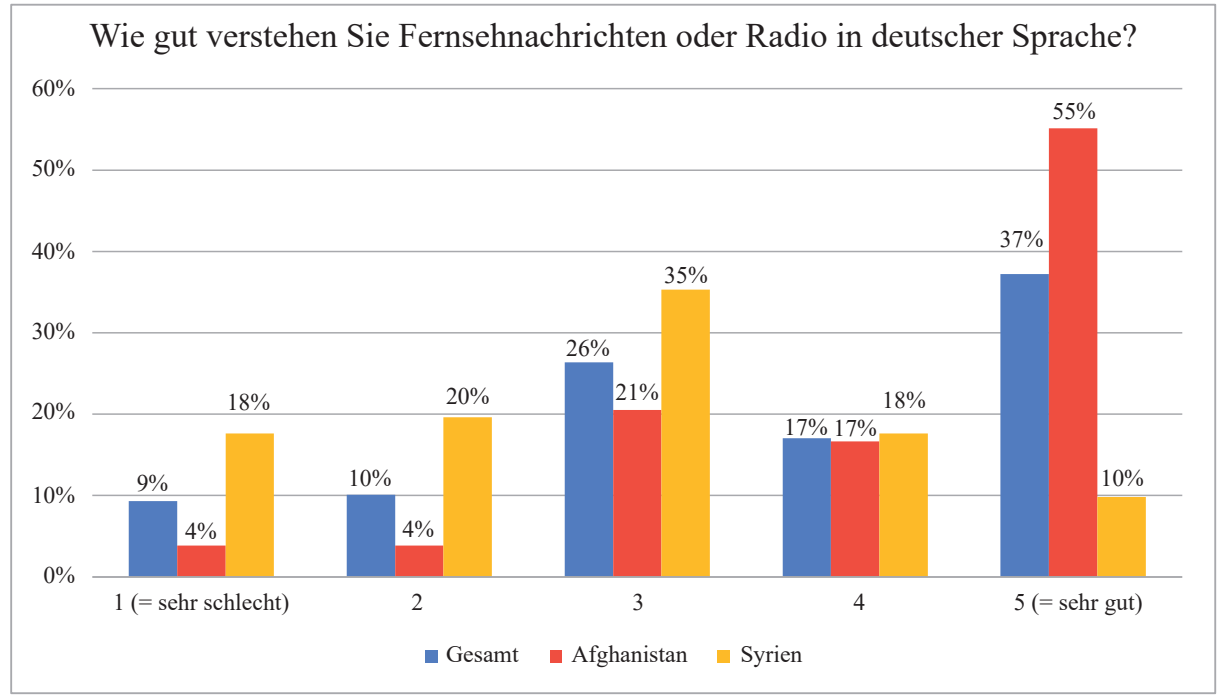

Quelle: eigene Erhebung 2020.

In Bezug auf den Asylstatus in Österreich (vgl. Abb. 1.9) dominierten in der Grundgesamtheit die Asylberechtigten (63\%) vor den subsidiär Schutzberechtigten (15\%) und den Asylwerber/inne/n (10\%). Nach Subgruppen aufgeschlüsselt waren bei den Afghan/inn/en (47\%) und vor allem bei den Syrer/inne/n (83\%) die Asylberechtigten vorherrschend. Unter den Afghan/inn/en befand sich auch ein beträchtlicher Anteil (24\%) subsidiär schutzberechtigter Personen, ein Status, der Afghan/inn/en viel öfter zugestanden wird als geflüchteten Syrer/innen, welchen zumeist Asylstatus gewährt

Abb. 1.9: Asylstatus (gesamt und nach Herkunft)

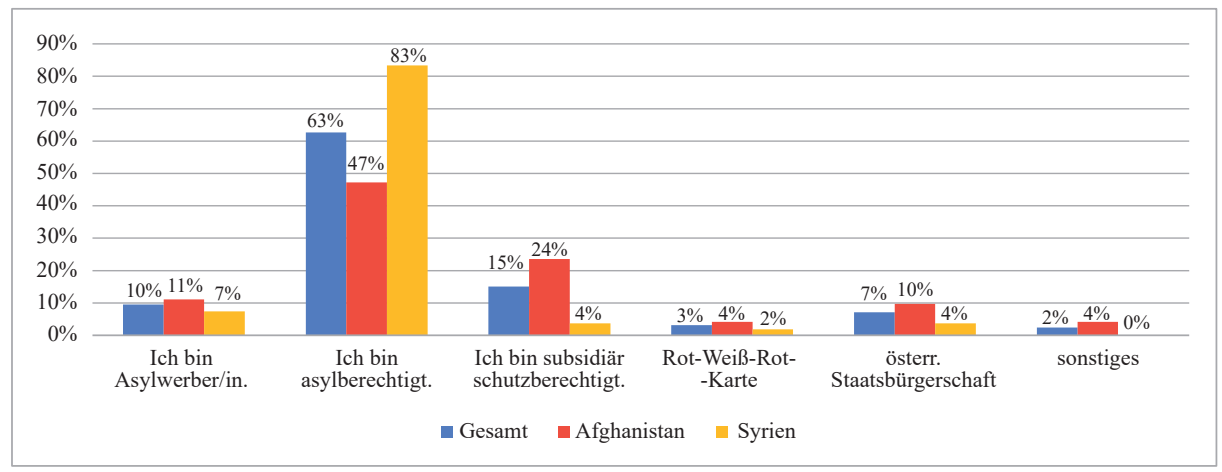

Quelle: eigene Erhebung 2020. 
wird. Acht Afghan/inn/en und vier Befragte aus Syrien hatten noch den Status von Asylwerber/inne/n inne. Wenige (v.a. Afghan/inn/en) verfügten über die Rot-Weiß-RotKarte und vor allem unter den Befragten aus Afghanistan waren 10\% bereits eingebürgert, bei den Syrer/inne/n hingegen nur 4\%.

Die Haushaltsstrukturen (vgl. Abb. 1.10.1) spiegelten eine erhebliche Bandbreite wider und unterscheiden sich zwischen den beiden Subgruppen deutlich. In summa dominierten Personen, die noch im Haushalt mit den Eltern wohnten (22\%) vor den alleinlebenden (19\%) Befragten. 17\% wohnten mit dem/r Ehepartner/in und 15\% mit Ehepartner/in und Kindern in einem Haushalt. Wohngemeinschaften waren mit 13\% vertreten und in Asylunterkünften lebten nur wenige.

Abb. 1.10.1: Haushaltsstrukturen (gesamt und nach Herkunft)

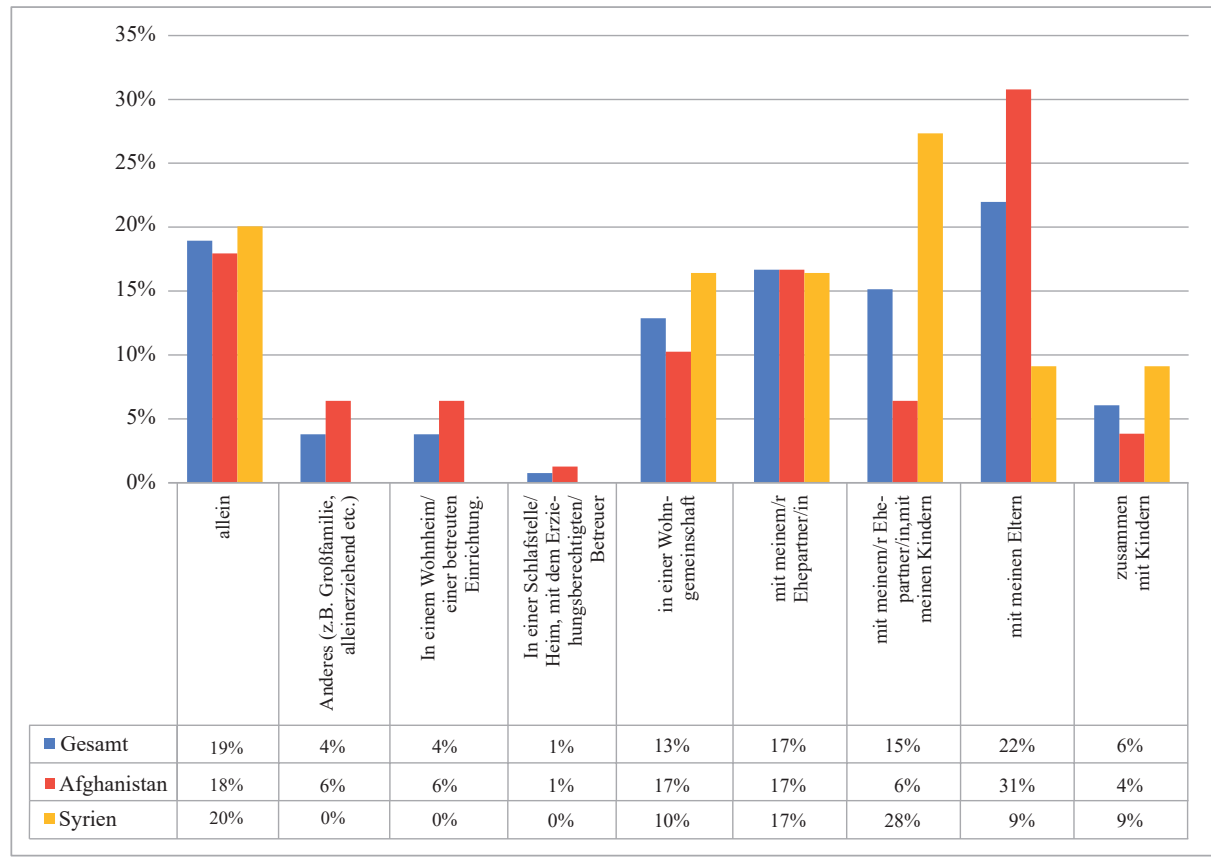

Quelle: eigene Erhebung 2020.

Bei den Geflüchteten aus Afghanistan wohnten besonders viele (31\%) noch im Haushalt der Eltern und nahezu gleich hoch war der Anteil jener Befragten, die mit $\mathrm{dem} / \mathrm{r}$ Ehepartner/in (17\%) oder alleine (18\%) lebten. Weitere 10\% wohnten in einer Wohngemeinschaft mit anderen Geflüchteten, eine gerade bei den jungen Personen, die aus Afghanistan geflüchtet sind, sehr verbreitete Wohnform (vgl. KoHLBACHER 2020a, b). Bei den Afghan/inn/en waren des Weiteren auch Großfamilien (6\%), Alleinerziehende oder Personen in einer betreuten Einrichtung (6\%) in geringeren Anteilen vertreten. 
Bei den Syrer/inne/n dominierten Familienhaushalte mit Kindern (28\%) und Alleinlebende (20\%), darüber hinaus waren Ehepaare (17\%) und noch häufiger als in der Subgruppe aus Afghanistan Wohngemeinschaften (17\%) vertreten. Nur ein vergleichsweise geringer Anteil der syrischen Respondent/inn/en lebte mit den Eltern im selben Haushalt (9\%). In betreuten Einrichtungen, also v.a. Asylquartieren, waren insgesamt nur wenige wohnhaft und darunter befanden sich fast ausschließlich Afghan/ inn/en (6\%).

Abb. 1.10.2: Haushaltsgrößen (gesamt und nach Herkunft)

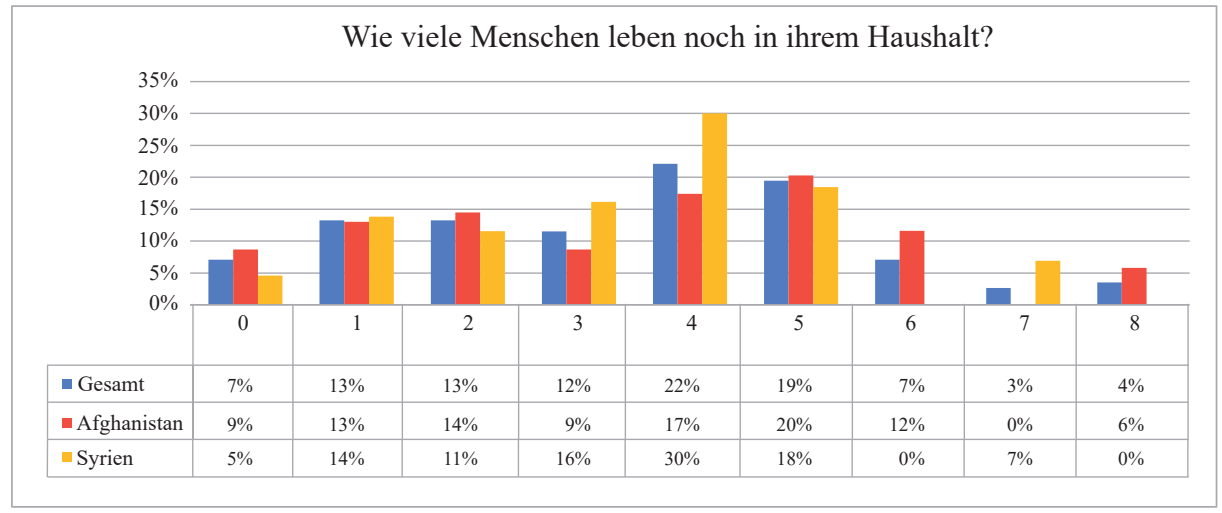

Quelle: eigene Erhebung 2020.

Aufschlussreiche Unterschiede ließen sich auch hinsichtlich der Größenstruktur der Haushalte feststellen (vgl. Abb. 1.10.2), wobei diese eine beträchtliche Bandbreite widerspiegelte. Bezogen auf alle Befragten dominierten größere Haushalte mit weiteren vier $(22 \%)$ oder fünf Personen (19\%). Bei den afghanischen Respondent/inn/en vor allem Haushalte mit weiteren fünf (20\%) bzw. vier (17\%) Personen. Bei den Syrer/ inne/n lebten vor allem vier (30\%) oder fünf weitere Personen (18\%) im selben Haushalt wie der/die jeweilige Befragte. Vierpersonenhaushalte traten bei den Syrer/inn/en mit $16 \%$ auf, bei weiteren $11 \%$ lebte noch eine weitere Person im selben Haushalt. Dreipersonenhaushalte (meist Ehepaare mit Kind, aber auch Wohngemeinschaften) waren in der afghanischen Subgruppe etwas häufiger als in der syrischen, unter ersteren war auch die Zahl der Singlehaushalte mit 9\% höher. In geringen Zahlen waren auch Großfamilienhaushalte bzw. größere Wohngemeinschaften in beiden Gruppen repräsentiert. Bei den Afghan/inn/en vor allem Haushalte mit 6 oder 8 weiteren Personen, in der syrischen Subgruppe Einheiten mit sieben weiteren Haushaltsmitgliedern.

Aufgrund der spezifischen Herausforderungen der Corona-Pandemie interessierte auch der wichtige Aspekt, inwieweit seitens der Befragten Betreuungsverpflichtungen für außerhalb des eigenen Haushalts lebende Angehörige bestanden (vgl. Abb. 1.11). In diesem Punkt haben 24\% mit ,ja“ geantwortet. Dabei manifestierte sich ein deutlicher 
Unterschied zwischen den beiden Herkunftsgruppen. So gab in beiden Communities die Mehrheit zwar an, keinen Betreuungspflichten nachkommen zu müssen, der Anteil jener mit solchen Pflichten war bei den Afghan/inn/en mit 27\% jedoch höher als bei den Befragten aus Syrien (20\%).

Abb. 1.11: Betreuungsverpflichtungen (gesamt und nach Herkunft)

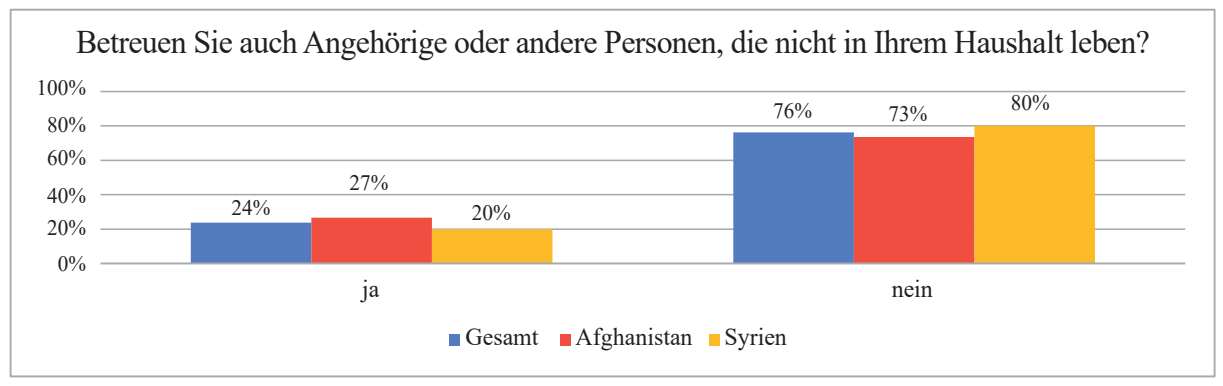

Quelle: eigene Erhebung 2020. 


\section{INFORMATIONSKANÄLE}

\subsection{Einholung von Informationen über COVID-19}

Abb. 2.1: Informationsstand über die Coronamaßnahmen (gesamt und nach Herkunft)

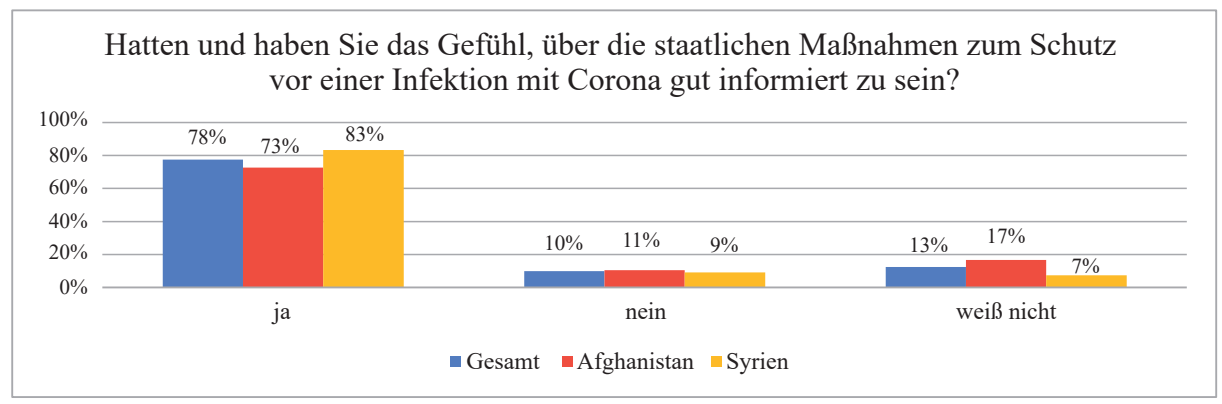

Quelle: eigene Erhebung 2020.

Betreffend die Compliance bezüglich der politischen Maßnahmen zur Minimierung von Infektionsrisiken und Übertragungswegen und $\mathrm{zu}$ den individuellen Schutzmaßnahmen waren die Informationssuche und -rezeption sowie die kritische Reflexion von Informationen über die Pandemie von besonderer Wichtigkeit (vgl. Abb. 2.1). Die Selbsteinschätzung des eigenen Informationsstandes über die auf COVID-19 bezogenen Maßnahmen der Regierung in der Vergangenheit sowie auch die Situation zum Erhebungszeitpunkt betreffend war durchwegs positiv. Nur insgesamt $10 \%$ der Befragten hatten nicht das Gefühl gut informiert zu sein, davon war der Anteil bei den Afghan/inn/en mit 11\% etwas höher als in den syrischen Communities (9\%). Ein vor allem bei den Afghan/inn/en deutlich höherer Anteil von 17\% war sich diesbezüglich nicht sicher. Von den Syrer/inn/en hatten nur 7\% die Kategorie ,weiß nicht““ angekreuzt.

Die überwiegende Mehrheit in beiden Gruppen hatte also das Gefühl durchwegs gut informiert zu sein. Jener geringe Teil der Respondent/inn/en, auf die das nicht zutraf, wurde einer tiefergehenden Analyse unterzogen (vgl. Abb. 2.1.1). Von ihnen klassifizierten $31 \%$ die offiziellen Informationen vor allem als lückenhaft (immerhin 36\% bei den Afghan/inn/en und nur 20\% bei den Syrer/inn/en) bzw. führten ihre Probleme hinsichtlich des Informationszugangs primär auf ihre geringen Deutschkenntnisse zurück (hier besonders die Syrer/inn/en mit 60\% gegenüber nur 9\% in den Communities aus Afghanistan). Unter den Befragten aus Afghanistan spielte mit 27\% auch mangelndes Vertrauen in offizielle, staatliche Informationen eine Rolle. Dies spiegelt die Situation im Herkunftsland Afghanistan sowie auch im benachbarten Iran wider, wo staatlichen Informationskanälen wenig Glauben geschenkt werden kann, da diese oftmals propagandistisch im Interesse des Regimes agieren. Mangelndes Wissen darüber, wo verlässliche Informationen zu erhalten gewesen waren, wurde von immerhin 27\% der Afghan/ inn/en als Begründung angegeben. Kein/e einzige/r Befragte/r aus Syrien hat dies 
angekreuzt. Nur wenige Respondent/inn/en aus Syrien und kein/e Afghan/e/in bekundeten eine gewisse Indifferenz. In summa belegen die Resultate ein hohes Ausmaß an Interesse an den auf COVID-19 bezogenen Maßnahmen.

Der Zugang zu verlässlichen Informationen, das Verstehen derselben und der Umgang mit den gebotenen Inhalten waren auch wichtige Punkte, die im Rahmen der qualitativen Interviews sowohl von den Expert/inn/en als auch den Respondent/inn/en aus den beiden Communities häufig angesprochen wurden. Jedenfalls bestand in der frühen Phase des Lockdowns ein beträchtliches Defizit an mehrsprachigen Informationsangeboten zu den Regierungsmaßnahmen und der an die Organisationen herangetragene Informationsbedarf musste unter großem Zeitdruck zu decken versucht werden. Mit längerer Dauer des Lockdowns verblieb der Informationsbedarf auf einem hohen Niveau und viele Menschen gingen zunehmend kritisch mit unterschiedlichen Informationsquellen um. Dies kann als ein positiver Nebeneffekt dieser herausfordernden Zeit bewertet werden. Der Erwerb einer höheren Sicherheit und Routine im Umgang mit digitalen Medien, die kritische Reflexion der (im deutschen Original wie auch in Übersetzung) verfügbaren Meldungen und Inhalte sowie die Auseinandersetzung mit den Auswirkungen der Krise für das persönliche Leben in den unterschiedlichen Sphären der sozialen und der Systemintegration zählten dazu. Kritisch wurde von vielen das anfängliche Manko beziehungsweise die lückenhafte Darstellung der Maßnahmen für Geflüchtete aufgeworfen. Bei ihnen hatte diese Vorgangsweise den Eindruck entstehen lassen, dass sie sich nicht angesprochen und wahrgenommen gefühlt haben.

Sogar Mitarbeiter/inne/n der NGOs fiel es mitunter schwer, den Überblick über die sich rasch ändernden Verordnungen und Maßnahmen zu bewahren. Wie auch bei den Österreicher/inne/n kam es auch bei nicht wenigen Befragten zu einer Art Informationsüberforderung bezüglich der schnell wechselnden Maßnahmen.

Auch für im medizinschen Bereich Tätige war die Informationsbeschaffung während der ersten Phase des ersten Lockdowns sehr schwierig. Da viele medizinische Einrichtungen geschlossen waren, mussten sich einzelne Akteur/inn/e/n erst einen Überblick verschaffen, wohin sie ihre Klient/inn/en verweisen konnten.

Der Informationsstand der meisten Angehörigen der Communities wurde von Expert/inn/en aus den NGOs unterschiedlich eingeschätzt. Im Laufe der Pandemie hat sich der Informationszugang für die einzelnen Personen wie auch seitens der Communities durch aufbereitete/übersetzte Informationen und aufsuchende (meist telefonische) Beratungstätigkeit sukzessive verbessert. Durch die Communities, vertreten durch Vereine, reale oder virtuelle/soziale Netzwerke, durch sogenannte Gatekeeper oder Multiplikator/inn/en wurde hier rasch ein alternatives Beratungs- bzw. Betreuungsangebot aufgebaut. Das Defizit an Informationen, welches seitens der befragten Personen zu Beginn des ersten Lockdowns bestand, war nicht primär abhängig vom Bildungsgrad, sondern lag an der Informationsvermittlung, die durch die herrschende Asylpolitik und populistische Maßnahmen gekennzeichnet war. Insofern waren die Aktionen, die im weitesten Sinne communitybasiert waren, sehr bemerkenswerte Beispiele für die Entwicklung einer Agency und den Aufbau von Selbstorganisationsstrukturen. 
Eine sehr bemerkenswerte Initiative war beispielsweise jene einer palästinensischen Ärztin in Wien, die aus eigenem Impuls eine „Taskforce“ gegründet hatte, die in Österreich tätig und auch in ganz Europa vernetzt war. Es waren Ärzt/inn/e/n, Sozialarbeiter/innen und auch Politiker/innen dabei. In etwa 200 Personen haben sich regelmäßig ausgetauscht, Fortbildungen online organisiert und sich vor allem, aber nicht ausschließlich für arabischsprachige Patient/inn/en eingesetzt. Wo notwendig hat man einander gegenseitig mit Informationsmaterial wie auch mit Medikamenten unterstützt, vor allem mit Ärztemustern. Die Ärztin hat Informationen der WHO, die auf Englisch vorhanden waren, weitergeleitet und in weiterer Folge auch für die Ärztekammer wichtige Informationen übersetzt. Diese Übersetzungen bezogen sich vor allem auf die Verhaltensregeln (Maskentragen, Arztbesuche), Hygienevorschriften, wie man Rezepte für benötigte Medikamente bekam etc. Diese hat sie über soziale Medien gepostet, die dann von Multiplikator/inn/en weitergeleitet wurden. Bereits sehr früh, Ende Februar 2020, hat sie mit solchen Übersetzungs- und Vernetzungstätigkeiten begonnen. Die Sprachbarriere(n) in der Informationsvermittlung waren allerdings nicht alleine bei den Geflüchteten festzustellen. Da diese Ärztin auch als Arbeitsmedizinerin für ein internationales Unternehmen tätig war, musste sie auch in diesem Feld mit Schwierigkeiten in der Informationsbeschaffung kämpfen. Sie wies darauf hin, dass auch die offiziell eingerichteten staatlichen Hotlines kein mehrsprachiges Personal zu Verfügung hatten und Informationen oder Beratung anfangs nicht einmal auf Englisch angeboten hatten.

Es haben sich keineswegs alle Befragten der afghanischen Community gut informiert gefühlt und gerade am Beginn der Pandemie wurden Meldungen über COVID-19 oftmals nicht ernst genommen. Dies resultierte vor allem aus den Erfahrungen mit der mangelnden Verlässlichkeit und der politischen Propaganda der Medienberichterstattung in den Herkunftsstaaten.

Die Syrer/innen konnten sukzessive auf mehrere übersetzte Quellen (ORFMeldungen, staatliche Maßnahmen) zurückgreifen, die über unterschiedliche soziale Medien geteilt wurden. Auch haben bereits Kinder, die bessere Sprachkenntnisse als die Eltern und einen selbstverständlicheren Zugang zu sozialen Medien haben, Übersetzungstätigkeiten für die ältere Generation übernommen. Des Weiteren konnte man auf die Erläuterungen und Erklärungen der Maßnahmen durch Verwandte oder Bekannte, die schon länger in Wien lebten, zurückgreifen. Herausfordernd war es - wie für alle den sich schnell ändernden Maßnahmen zu folgen. Durch die Übersetzungstätigkeiten konnte vor allem in den syrischen Communities wieder ein Vertrauen in staatliche Maßnahmen und eine Compliance geschaffen werden.

Die unterschiedlichen arabischen Communities und Vereine haben zusätzlich zu den übersetzten Informationen auch eigene Beiträge gestaltet (u.a. Videos). Durch das zunehmende Angebot konnte ein kritischer Medienkonsum erzeugt werden, wobei von Expert/inn/en wie auch Respondent/inn/en auf die unterschiedlich politisch motivierten arabischen Medienberichte verwiesen wurde, die es nun kritisch zu hinterfragen und zu vergleichen galt. 
Abb. 2.1.1: Begründungen für mangelnde Informationen (gesamt und nach Herkunft)

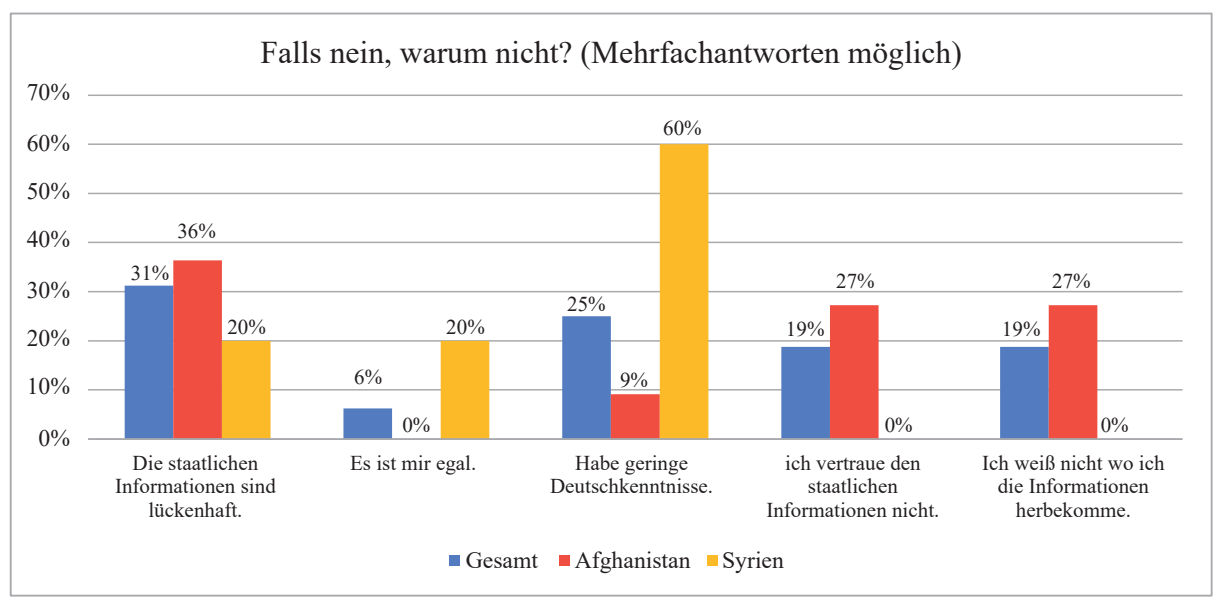

Quelle: eigene Erhebung 2020.

Es war für einige Personen in den afghanischen Communities, vor allem bedingt durch Bildungsdefizite und die ungewohnte Auseinandersetzung mit medialer Berichterstattung, sehr schwierig, sich mit Informationen kritisch und selektiv auseinanderzusetzen. Falschinformationen und abstruse Fake News spielten also ebenfalls eine nicht unwesentliche Rolle.

Von entscheidender Wichtigkeit war vor allem für jene Geflüchteten mit geringen Deutschkenntnissen die Übersetzung der Informationen in die Muttersprachen FarsiDari, Paschto und Arabisch. Die Verfügbarkeit von Informationen in vielen Herkunftssprachen wurde sowohl von den Expert/inn/en als auch den Befragten aus den Communities als positiver Nebeneffekt der Pandemie gewertet.

Die Übersetzungen ${ }^{9}$ wurden von den verschiedenen Communities schriftlich wie auch mündlich in Videoform produziert; auch österreichische Organisationen, die Unterkünfte für Geflüchtete betrieben (z.B. Rotes Kreuz) haben in Folge Übersetzungen und Videoproduktionen in Auftrag gegeben. Auch die Stadt Wien hatte eine mehrsprachige Hotline eingerichtet, die dann von manchen sehr positiv aufgenommen wurde.

Seitens einzelner Expert/inn/en herrschte eine gewisse Uneinigkeit darüber, ob österreichische Medien über TV generell wirklich in größerem Ausmaß konsumiert wurden. Nicht alle konnten und können österreichisches Fernsehen in dem Ausmaß empfangen und für macne waren Apps und Newsletter von Organisationen scheinbar wichtiger. Für religiöse Vertrauenspersonen war allerdings vorwiegend die telefonische Kontaktaufnahme am wichtigsten, um Betreuung in Glaubensfragen etc. anzubieten.

$9 \quad$ siehe beispielsweise eine mit arabischen Untertiteln gepostete Pressekonferenz der Regierung $<$ https://www.youtube.com/watch?v=E44iZg9aNyU $>$. 
Einer der wenigen positiven Nebeneffekte der Krise bestand darin, dass in bestimmten Branchen (v.a. Lebensmittelhandel, Lagerbereich, Agrarsektor) kurzfristig viele zusätzliche Arbeitskräfte benötigt wurden. Auch diese Informationen wurden über die NGOs an die afghanischen und syrischen Communities weitergegeben. Den Aussagen der Befragten gemäß waren die meisten Bewerbungen in diesen Sektoren jedoch letztlich nicht erfolgreich. Gerade in diesem Bereich sehen Organisationen auch eine Möglichkeit sich in Zukunft vermehrt einzubringen und Hilfestellungen bei Informationsweitergabe, bei Bewerbungen etc. zu leisten.

Dass das Misstrauen gegenüber amtlichen Informationen nicht immer ganz ungerechtfertigt war, bekundeten sowohl Geflüchtete als auch Expert/inn/en, denn leider wurden sogar von einzelnen offiziellen Stellen und Flüchtlingseinrichtungen nur bedingt richtige oder sogar falsche Informationen verbreitet, was seitens der Communities sehr emotional kritisiert wurde. Manche haben dahinter eine Taktik der Angstverbreitung gesehen. Dadurch wurde das Vertrauen in die Maßnahmen beschädigt. Als in der Folge Privatpersonen versuchten Übersetzungen richtigzustellen oder diese vollständig anzubieten, waren unbeabsichtigt auch verschiedene Fehlinformationen verbreitet worden. Diese und die rigiden Erteilungen von Verwaltungsstrafen aufgrund von Übertretungen des Ausgehverbots (s.u.) ließen so manche die Willkür staatlicher Machtstrukturen erfahren. Manche Befragte waren davon sehr überrascht, da sie dies in einer solchen Form in Österreich bis dahin nicht erwartet hatten.

Die palästinensische Ärztin, die eine Hotline für arabischsprachige Personen eingerichtet hatte, erwähnte auch die Problematik des Informationsflusses, wenn es zu heiklen Situationen kam, wenn etwa Kinder erkrankten oder wenn Personen stationäre Krankenhausaufenthalte oder fachärztliche Betreuung benötigten. Viele Institutionen (Ordinationen, Spezialeinrichtungen, psychosoziale Dienste) waren geschlossen und die Klient/inn/en hatten keine Ahnung, an wen sie sich wenden konnten. Auch die sogenannten Gatekeeper im System, die Betreuungseinrichtungen im Fluchtbereich, hatten während des ersten Lockdowns geschlossen. Menschen, die das Gesundheits-, Rechts- und Sozialwesen in Österreich noch nicht so kannten, wussten also nicht, wo sie entsprechende Hilfe finden konnten. Auch die Ärzt/inn/e/n der Hotline waren überfragt, wenn es beispielsweise um medizinische Spezialfälle ging. Vor allem war auch die Intersektionalität von medizinischen und rechtlichen Fragen in manchen Krisenfällen schwierig und erzeugte bei den Betroffenen Angst.

\subsection{Informationen über COVID-19 und Deutschkenntnisse}

Ein Konnex der Nutzung von bestimmten Informationsquellen mit den Deutschkenntnissen ist klar erkennbar. Österreichische Printmedien wurden von keinem einzigen Befragten mir sehr schlechten Deutschkenntnissen genutzt, dafür von jenen mit den besten Deutschkompetenzen zu jeweils 12,5\% täglich oder mehrmals wöchentlich und zu fast 22\% alle paar Wochen. Auch Befragte mit guten Deutschkenntnissen griffen 
häufig zu österreichischen Zeitungen (11,5\% täglich, 26,9\% mehrmals wöchentlich und $11,5 \%$ einmal wöchentlich). Simultan zu schlechteren Deutschkenntnissen nimmt auch die Häufigkeit der Nutzung von deutschsprachigen Printmedien deutlich ab.

Ganz ähnlich wie im Fall der Zeitungen fiel die Nutzung des Nachrichtenangebotes des ORF aus. Wer über sehr gute bzw. gute Deutschkompetenz verfügte, nutzte den ORF zu 17,1 bzw. 13,6\% täglich bzw. sogar mehrmals täglich (11,4 und 13,6\%). Nur 20\% der Geflüchteten mit sehr guten Deutschkenntnissen haben den ORF gar nicht genutzt sowie 18,2\% jener mit guter Deutschkompetenz. Wer Deutsch schlecht oder eher schlecht verstand, nutzte direkte ORF-Beiträge entweder gar nicht oder nur alle paar Wochen. Anders war die Verteilung bei den Social Media. Diese wurden auch von Geflüchteten mit eher geringen Deutschkompetenzen zu mehr als 30\% mehrmals täglich sowie von 15,4\% mehrmals wöchentlich genutzt. Auch jene mit besseren Deutschkenntnissen bezogen Coronainformationen gerne aus den Social Media. Jene mit den besten Deutschkompetenzen zu 28,6\% mehrmals täglich, zu 17,1\% mehrmals wöchentlich und zu jeweils 14,3\% einmal pro Woche oder alle paar Wochen. Auch bei Personen mit mittleren Deutschkenntnissen ragten Social Media als wichtige Informationsquellen hervor. Am geringsten war ihre Nutzung bei jenen mit der geringsten Deutschkompetenz, von denen zwei Drittel keine Informationen zu Corona über diese Quelle einholten. In den sozialen Medien wurden aber übersetzte ORF-Beiträge geteilt; dies ist hinsichtlich der Einschätzung der Fragestellungen über Informationsfluss und Vertrauen in die Medien zu berücksichtigen. Die Informationen über den Konsum und das Vertrauen schienen anfangs widersprüchlich. Aus den qualitativen Interviews ging jedoch die Bedeutung der Übersetzungen von ORF-Beiträgen, die über unterschiedliche Kanäle geteilt wurden, hervor.

Satellitenkanäle wurden ebenfalls von Befragten mit den geringsten Deutschkenntnissen gar nicht gesehen, aber auch 69,2\% der Befragten mit sehr guter Deutschkompetenz nutzten diese gar nicht. In den Kategorien mit mittleren Deutschkenntnissen wurden Satellitenkanäle am häufigsten genutzt, nämlich von 33\% bzw. 25\% mehrmals wöchentlich. Verbreitet war auch die Nutzung alle paar Wochen, nämlich von $25 \%$ mit eher schlechten Deutschkenntnissen, aber auch von rund einem Fünftel jener mit den besten Deutschkompetenzen.

Die Internetseiten von österreichischen Vereinen und NGOs wurden eher von Respondent/inn/en mit mittlerer Deutschbeherrschung (Kategorien 3-4) als Informationsquellen herangezogen. Fast $61 \%$ nutzten diese mehrmals wöchentlich und $36,7 \%$ einmal pro Woche. Wer Deutsch exzellent beherrscht, hat die Informationen zur Pandemie ebenfalls häufig über NGOs bezogen. Jeweils 16,7\% mehrmals wöchentlich oder alle paar Wochen sowie zu 14\% einmal pro Woche.

Mit besseren Deutschkenntnissen sinkt die Bedeutung der Nutzung von Medien aus dem Herkunftsland. Wer exzellent Deutsch spricht, nutzt diese zu jeweils 43,3\% nur mehr alle paar Wochen oder auch gar nicht, wer eher geringe Deutschkompetenz aufweist aber zu 30\% täglich, zu 10\% mehrmals täglich und zu 20\% mehrmals wöchentlich. 
Etwas seltener ist bereits die Nutzung in der Gruppe mit mittlerer Deutschbeherrschung: Jeweils 5,9\% haben TV und andere Medien aus dem Herkunftsland täglich bzw. mehrmals täglich genutzt, 35,5\% mehrmals pro Woche und 11,8\% einmal wöchentlich.

Am seltensten wurden private Webseiten und Blogs der eigenen Communities von jenen in Anspruch genommen, die über die besten Deutschkenntnisse verfügen, nämlich von fast $60 \%$ gar nicht und von nahezu $30 \%$ alle paar Wochen. Personen mit sehr geringer Deutschkompetenz nutzten diese jedoch zu 50\% täglich und zu weiteren $50 \%$ alle paar Wochen. Auch Befragte mit mittlerer Deutschbeherrschung haben Blogs in Farsi-Dari, Paschto oder Arabisch zu 40\% täglich genutzt und zu 26,7\% zumindest alle paar Wochen.

Familienangehörige, die noch im Ausland leben, zählen vor allem für jene Befragten mit geringeren Deutschkenntnissen zu den häufig kontaktierten Informant/ inn/en. Jeweils 37,5\% der Respondent/inn/en kontaktierten Verwandte täglich oder mehrmals pro Woche, 12,5\% alle paar Wochen. Jene mit der besten Deutschbeherrschung in keinem Fall täglich und nur zu 3,6\% mehrmals wöchentlich, dafür aber zu $57,1 \%$ nur in großen Intervallen von einigen Wochen. Etwas höher waren die Anteile der täglichen Kontaktierung bei Personen mit eher guten (19,5\%) bzw. mittleren Deutschkenntnissen $(15,4 \%)$.

Freunde oder Bekannte aus den eigenen Communities spielten naheliegenderweise vor allem bei den Respondent/inn/en mit geringer Deutschkompetenz die größte Rolle. Personen mit eher geringer Deutschbeherrschung haben diese zu 55,6\% mehrmals wöchentlich um Informationen gebeten, dies taten nur 7,4\% derjenigen mit den besten Deutschkenntnissen sowie 5,3\% der Befragten mit eher guter Beherrschung der deutschen Sprache. Aufschlussreich war auch die Analyse der Kategorie ,gar nicht“. Mehr als die Hälfte $(51,9 \%)$ der Befragten mit den besten Deutschkenntnissen und $36,8 \%$ jener mit eher guter Deutschbeherrschung haben Freunde oder Bekannte aus den eigenen Communities zwecks Informationen über COVID-19 gar nicht kontaktiert.

Die Deutschbeherrschung war die wesentliche Determinante der Informationseinholung über österreichische Freunde und Bekannte. Wer am wenigsten des Deutschen mächtig war, hat nie Informationen über österreichische Netzwerke eingeholt, dies galt auch für 50\% jener mit eher geringer Deutschbeherrschung. Dies entspricht auch der Problematik, wie sie von Expert/inn/en, die in NGOs arbeiten, dargestellt wurde, dass sich ihre Klient/inn/en nämlich sukzessive zurückzogen, da anfangs nur Telefonkontakte und wenig Dolmetschdienste angeboten wurden.

Exzellente Deutschkenntnisse führten dazu, dass zu jeweils 13,3\% Coronainformationen entweder einmal oder mehrmals wöchentlich erfragt wurden, 30\% haben dies alle paar Wochen getan.

Die Mitarbeiter von NGOs spielten auch bei den beiden Subgruppen mit der besten Deutschbeherrschung als Informationsquellen zu COVID-19 eine eher untergeordnete Rolle. Am wichtigsten waren sie für jene mit eher schwachen oder mittleren Deutschkompetenzen. Von diesen Befragten haben 35\% mehrmals täglich oder wöchentlich 
die NGO-Mitarbeiter/innen kontaktiert, 33,8\% jener Respondent/inn/en mit mittlerer Deutschbeherrschung machten dies alle paar Wochen.

Interessant ist, dass der Zusammenhang zwischen der Deutschbeherrschung und der Informationseinholung über die Organisationen der jeweiligen Communities nicht sehr markant ausgeprägt ist. Auch die Befragten mit den schlechtesten Deutschkenntnissen gaben zu 100\% an, die Vereine nicht um Informationen gebeten zu haben. Jene mit der besten Deutschkompetenz haben die Vereine zu 42,9\% alle paar Wochen sowie zu 14,3\% mehrmals wöchentlich kontaktiert. Eine sehr geringe Rolle spielten die Vereine für jene mit eher guter Deutschbeherrschung (Level 4), die zu 70\% die Vereine nicht kontaktiert haben.

Abb. 2.2: Einschätzung des Informationsstandes über staatliche Maßnahmen für Arbeitnehmer/innen und Selbständige (gesamt und nach Herkunft)

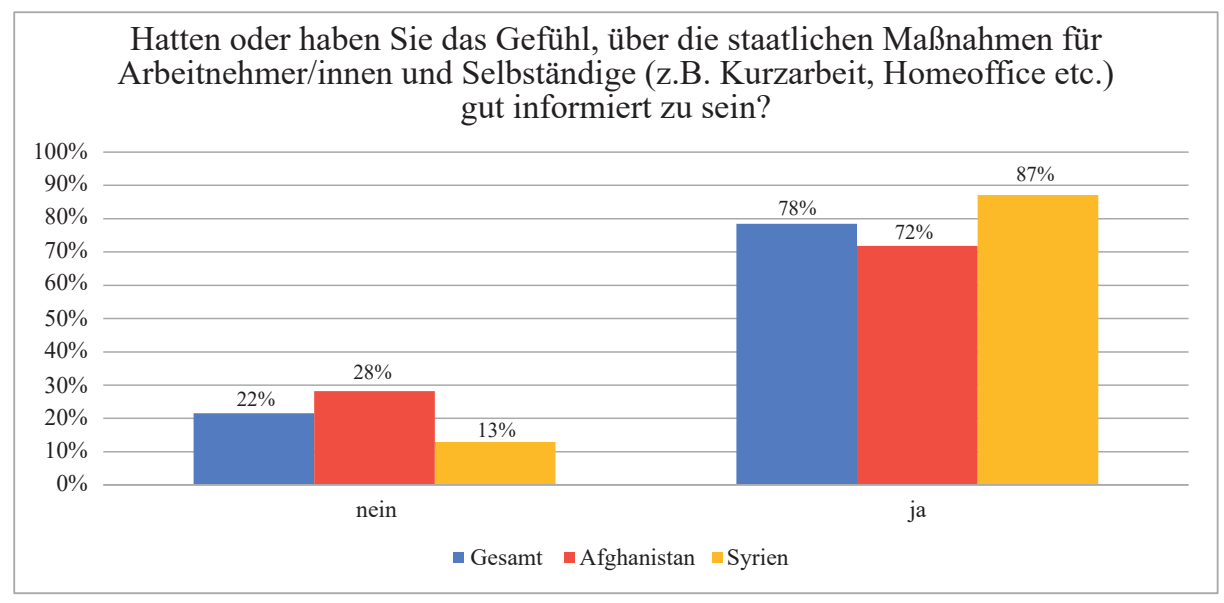

Quelle: eigene Erhebung 2020.

Eine spezielle Kategorie bilden die Informationen über vielfältige staatliche Hilfsmaßnahmen für Arbeitnehmer/innen und Selbstständige zur Bewältigung der Pandemiekrise (vgl. Abb. 2.2). Auch in dieser Hinsicht schätzte sich jeweils das Gros der Befragten in beiden Communities (72\% der Afghan/inn/en und $87 \%$ der syrischen Befragten) als gut informiert ein. Unter den Afghan/inn/en war der Anteil jener Befragten, welche dies verneinten $(28 \%)$, aber beträchtlich höher als in der Vergleichsgruppe aus Syrien (13\%). 
Abb. 2.2.1: Kausalfaktoren unzureichender Informationen über staatliche Maßnahmen für Arbeitnehmer/innen und Selbständige (gesamt und nach Herkunft)

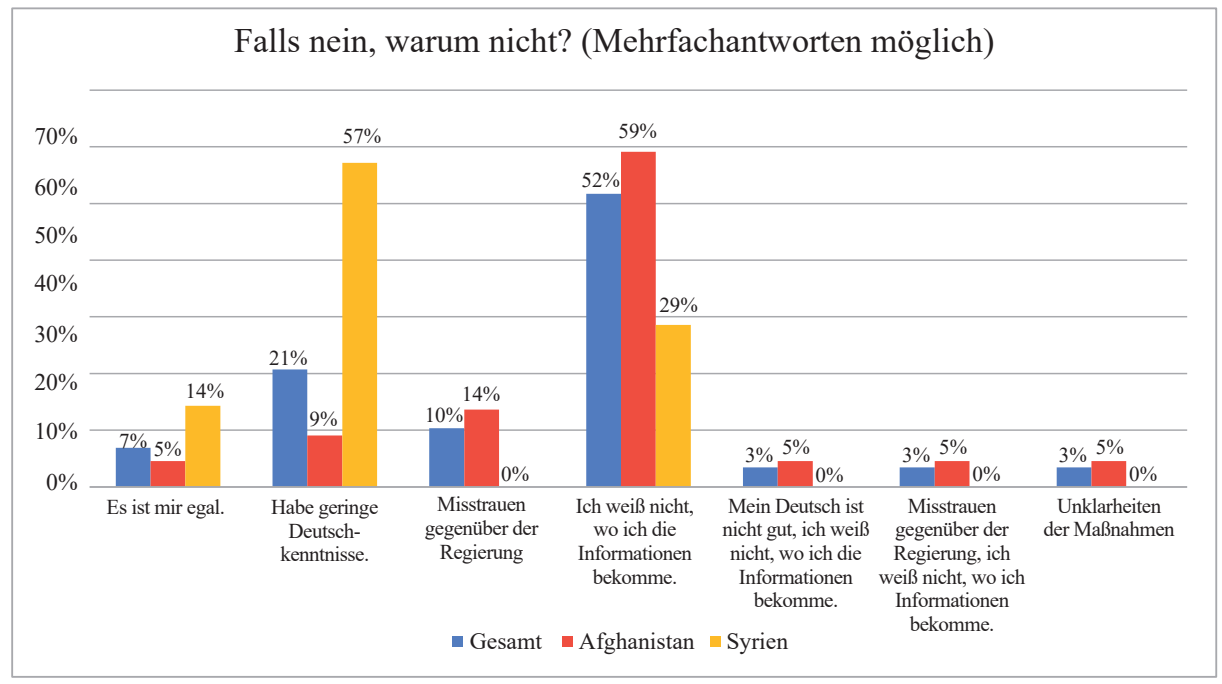

Quelle: eigene Erhebung 2020.

Zur vertiefenden Analyse dieser Probleme des Informationszugangs für einen Teil der Befragten wurden daher auch die Kausalfaktoren abgefragt (vgl. Abb. 2.2.1). Dabei zeigten sich beträchtliche Unterschiede zwischen den beiden Communities. Im Fall der Befragten aus Syrien stellten in erster Linie die geringen Deutschkenntnisse die größte Hürde dar (57\%), in zweiter Hinsicht auch das mangelnde Wissen darüber, woher Informationen zu beziehen gewesen wären (29\%), sowie seltener (14\%) ein gewisses Desinteresse. Unklarheiten hinsichtlich der Maßnahmen oder auch ein Misstrauen gegenüber der Regierung wurden seitens der syrischen Befragten gar nicht angeführt. Bei afghanischen Respondent/inn/en wurde eine größere Bandbreite an Gründen verbalisiert. Die Ursachen für das Gefühl mangelnder Information lagen in erster Linie am Unwissen über Informationsquellen (59\%), des Weiteren an einem generellen Misstrauen gegenüber der Regierung (14\%) sowie an geringen Deutschkenntnissen (9\%). Des Weiteren wurden in wenigen Fällen auch Unklarheiten in Bezug auf die Maßnahmen angeführt.

Abbildung 2.2.1 dokumentiert die Begründungen jener Respondent/inn/en, die angegeben hatten, über die Maßnahmen zur Unterstützung von Arbeitnehmer/inn/en nicht gut informiert gewesen zu sein. Mit 52\% dominiert die Begründung, dass man nicht gewusst hätte, woher die Informationen zu beziehen gewesen wären, danach folgt die Hürde geringer Deutschkenntnisse mit 21\%. 10\% begründeten ihren mangelnden Zugang zu Informationen mit einem Misstrauen der Regierung gegenüber und 7\% war es gleichgültig. 
Seitens der Befragten aus Afghanistan dominierte mit 59\% das Nichtwissen über Informationsquellen vor dem Misstrauen gegenüber der Regierung mit 14\%. Unter syrischen Befragten ragen die geringen Deutschkenntnisse mit 57\% als wichtigster Faktor hervor, danach folgen mit $29 \%$ die Unkenntnis bezüglich der Informationsquellen sowie die Begründung ,es ist mir egal“" mit 14\%.

\subsection{Informationsquellen}

Abb. 2.3: Informationsquellen und deren Nutzungshäufigkeiten (gesamt)

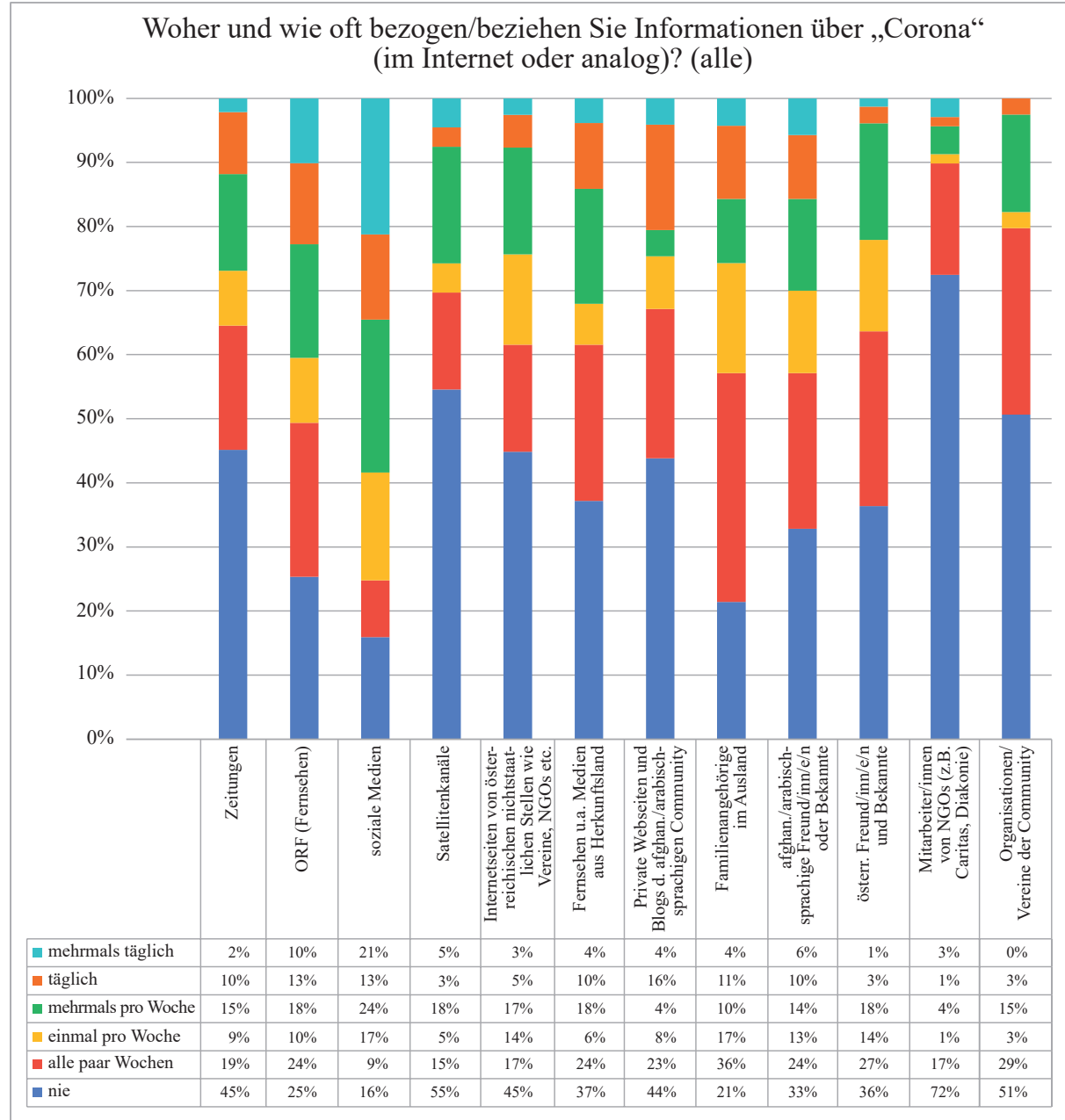

Quelle: eigene Erhebung 2020. 
Abb. 2.3 dokumentiert, dass die Befragten aus beiden Gruppen ihre Informationen über COVID-19 aus zahlreichen unterschiedlichen Informationsquellen bezogen, wobei bezüglich der Häufigkeiten der Inanspruchnahme vor allem Social Media, Printmedien und ORF-Programme im Vordergrund standen. Soziale Medien wurden von 21\% sogar mehrmals täglich bzw. von $13 \%$ täglich zur Informationsbeschaffung herangezogen. Ebenfalls beträchtlich waren die Anteile jener, die sich einmal (17\%) oder mehrmals wöchentlich (24\%) über Soziale Medien informierten. Häufig war weiters die Informationsbeschaffung über Printmedien, die täglich (10\%), mehrmals wöchentlich (15\%), einmal pro Woche $(9 \%)$ oder auch nur aber alle paar Wochen $(19 \%)$ zu Rate gezogen wurden. Ein weiteres wichtiges Informationsmedium waren Sendungen des ORF, die mehrmals täglich (10\%), täglich (13\%), mehrmals in der Woche $(18 \%)$ oder einmal pro Woche $(10 \%)$ gesehen wurden.

Weniger häufig (55\% der Befragten antworteten hier mit ,gar nie“) wurden Informationen aus den diversen Satellitenkanälen der Herkunftsländer oder über die Vereine der ethnischen Communities (51\% gar nie) bezogen. Bezüglich der Nutzung der Satellitenkanäle haben 5\% mehrmals täglich, 3\% einmal täglich, 18\% mehrmals die Woche und $5 \%$ einmal pro Woche angegeben. Private Webseiten und Blogs der eigenen Community sowie Familienangehörige im Ausland waren ebenfalls wichtige Quellen für Informationen mit Bezug zu Corona. Erstere wurden von $20 \%$ ein- oder mehrmals täglich genutzt, zweitgenannte zu 15\% in derselben Häufigkeit. Sehr relevant war auch die Informationsbeschaffung über Freund/inn/e/n aus der eigenen Community. Täglich oder sogar mehrmals täglich wurden von diesen Informationen von 16\% der Befragten bezogen. Mehrmals wöchentlich wandten sich $14 \%$ an ihre Freund/inn/e/n und 13\% einmal pro Woche. Wichtige Informationen wurden auch von österreichischen Bekannten oder Freund/inn/en bezogen. Diese wurden von 7\% täglich kontaktiert, von 18\% mehrmals wöchentlich bzw. von 14\% einmal pro Woche. Nur 33\% haben ihre Freund/inn/e/n aus der eigenen Community nie bezüglich Informationen gefragt und 36\% haben niemals österreichische Kontaktpersonen um Informationen bezüglich Corona gebeten. Vor allem in größeren Intervallen, also alle paar Wochen oder einmal pro Woche, haben viele Befragte Informationen von Familienangehörigen im Herkunftsland oder Freund/inn/en in der eigenen Community oder auch österreichischen Freund/inn/e/n eingeholt.

Aufschlussreich ist die spezielle Betrachtung der Antwortoption „nie“, also in welchem Ausmaß die jeweiligen Quellen gar nie zur Informationsgewinnung herangezogen worden waren. Diese zeigt einen Spitzenwert von $72 \%$ hinsichtlich der Mitarbeiter/inne/n von NGOs, was angesichts des laut Expert/inn/en sehr breiten Angebots (vor allem auch muttersprachlich) erstaunlich ist. Da wir keine Informationen über die Situation vor den Lockdowns haben, kann auch angenommen werden, dass dies mit der erzwungenen Schließung von Beratungseinrichtungen zusammenhängen kann.

Jeweils $45 \%$ der Befragten haben niemals Zeitungen bzw. Internetseiten nichtstaatlicher Stellen herangezogen. Von 55\% wurden Satellitenkanäle als Informationsquellen nie genutzt, TV-Kanäle oder andere Medien aus dem Herkunftsland haben 37\% nicht in Anspruch genommen. Dasselbe galt für private Webseiten und Blogs der afgha- 
Abb. 2.3.1: Informationsquellen und deren Nutzungshäufigkeiten (Herkunft Afghanistan)

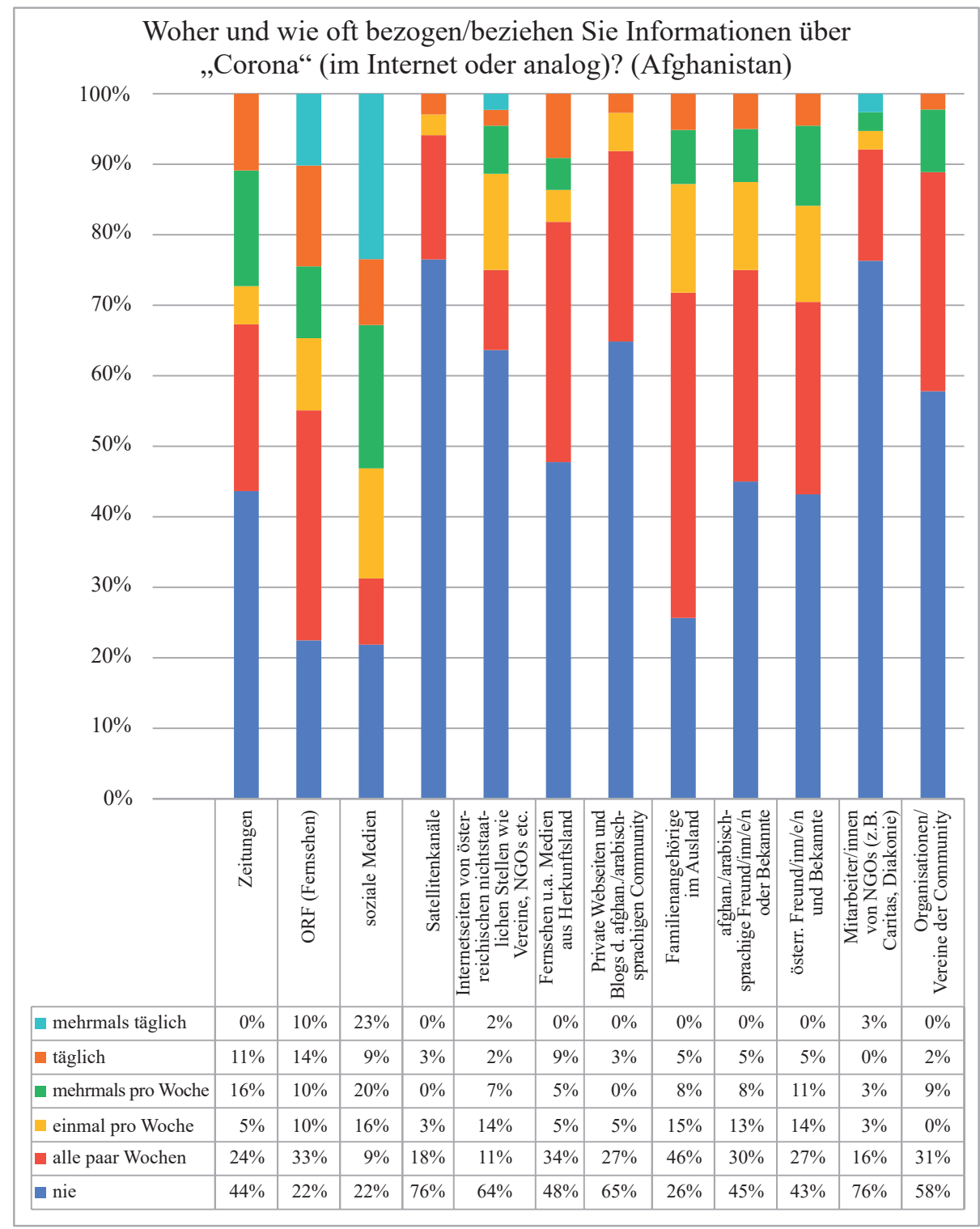

Quelle: eigene Erhebung 2020.

nischen bzw. arabischsprachigen Communities für 44\%. Im Vergleich dazu haben nur $25 \%$ nie Informationen über den ORF oder $16 \%$ nie über soziale Medien gewonnen. 
Aufschlussreich ist zunächst, welche Informationsquellen in der afghanischen Herkunftsgruppe am wenigsten genutzt wurden (vgl. Abb. 2.3.1). Mitarbeiter/innen von NGOs sowie Satellitenkanäle wurden zu 76\% niemals zur Informationsbeschaffung herangezogen. $\mathrm{Zu}$ 65\% bzw. 64\% wurde private Webseiten und Blogs der Communities sowie Internetseiten von österreichischen nichtstaatlichen Stellen nie genutzt. Einen hohen Wert bezüglich der Nichtnutzung vorhandener Angebote erreichten auch die Vereine der Communities mit 58\%. Mit Werten zwischen 43 und 45\% haben die Befragten aus Afghanistan niemals Zeitungen, Freund/inn/e/n aus der Community sowie österreichische Freunde zu Corona-Informationen befragt. Wichtige Optionen der Informationsrezeption waren ORF-Sendungen und Printmedien im Allgemeinen. Die geringsten Werte hinsichtlich der Nichtnutzung entfielen mit jeweils $22 \%$ auf den ORF und Social Media sowie mit 26\% auf Familienangehörige im Ausland. Diesem Bild entspricht auch die Analyse jener Quellen, aus denen die Informationen in erster Linie bezogen wurden. Von 23\% wurden mehrmals täglich soziale Medien und von 10\% der ORF konsultiert. 19 bzw. 14\% haben sich aus diese beiden Quellen täglich informiert. Mehrmals in der Woche haben 20\% in den Sozialen Medien und 10\% über den ORF Informationen eingeholt. $11 \%$ haben täglich Printmedien konsumiert und $18 \%$ taten dies mehrmals in der Woche bzw. 5\% zumindest einmal wöchentlich. Einen nennenswerten Anteil von 9\% der täglichen Nutzung erreichten daneben noch Fernsehen und Medien aus dem Herkunftsland. Deutlich seltener wurden die Angebote der Vereine und Organisationen der afghanischen Communities genutzt. 31\% haben nur alle paar Wochen Informationen über diese bezogen.

Dies überrascht, da doch vor allem im Bereich der Informationszusammenstellung, der -koordination und -diffusion die Mitarbeiter/innen und Repräsentant/inn/ en der Vereine und NGOs der Flüchtlingsbetreuung sowie die Vereine der afghanischen Communities während und auch noch nach dem Lockdown ungeheuer viel an Arbeit geleistet haben. Eventuell könnte diese überraschende Zahl auch damit zusammenhängen, dass sehr viele Informationen über Soziale Medien geteilt wurden und man oft nicht über die Ursprungsquelle Bescheid wusste oder Informationen diesen zuordenbar waren. Eine besondere Herausforderung bestand in den kurzen „Halbwertszeiten“ der meisten Informationen und auch der geltenden Maßnahmen, die sich sehr rasch änderten und mitunter auch nicht klar formuliert bzw. kommuniziert wurden. War es schon für Österreicher/innen ohne Migrationshintergrund schwierig, einen Überblick zu bewahren, waren für Afghan/inn/en mit oft geringen Deutschkenntnissen die damit verbundenen Herausforderungen noch erheblich größer. Relevant für diese Informationen war auch der Erhebungszeitraum. Die Respondent/inn/en beriefen sich auf die Situation während des ersten Lockdowns, während die qualitativen Interviews und Befragungen der Vereine dann ein wenig zeitversetzt im Sommer stattgefunden haben. Relativ kurzfristig sind eine Reihe von Initiativen umgesetzt worden, um Informationen $\mathrm{zu}$ verbreiten und alternative Unterstützungsangebote $\mathrm{zu}$ entwickeln.

Vertrauensbildende Maßnahmen waren dann direkte (telefonische) Kontaktaufnahmen mit den Klient/inn/en. Manche NGOs haben dies auch mit Dolmetscher/ 
inne/n bewerkstelligt. Wichtig waren auch Informationen wie Notfallsnummern, die über soziale Medien geteilt wurden. Schließlich wurden dann auch bereits während des ersten Lockdowns psychologische Informationen übersetzt und geteilt.

Im Rahmen der Informationsweitergabe spielten die Vernetzungen der NGOs untereinander sowie mit Institutionen des Gesundheitswesens eine entscheidende Rolle. Die Vereine haben die Informationen dann auch speziell auf ihre Klient/inn/en zugeschnitten und aufbereitet, wie zum Beispiel für afghanische Jugendliche. Die in Österreich verzweigten Vereine der afghanischen Communities konnten die relevanten Informationen über ein Netz an Zweigvereinen im gesamten Bundesgebiet verbreiten.

Die Informationen wurden zwar primär digital verbreitet, wichtig waren aber auch die Weitergabe von relevanten Telefonnummern und die privaten Informationsflüsse. Es gab wenige Alternativen zu den Online-Formaten. Seitens der NGOs und Vereine wurden als Mutiplikator/inn/en meist auch zahlreiche der schon zuvor in vielen Bereichen engagiert tätigen Ehrenamtlichen in die Informationsverbreitung involviert. So herausfordernd die Situation auch war, dennoch positiv gesehen wurden von manchen Expert/inn/en die Auswirkungen auf den Integrationsbereich. Hierbei wurden vor allem die Informationserstellung und -beschaffung, die Sensibilisierung und kritische Bewertung, das Aufkommen einer Agency insbesondere im Bereich der online zur Verfügung gestellten (Weiter-)Bildungsmöglichkeiten und Maßnahmen auf dem Arbeitsmarkt erwähnt.

Hierbei relevant war einerseits, wie und ob es eine Vernetzung (Vereine, soziale Medien) bereits vor der Pandemie gab. Für eine Betreuung seitens der österreichischen NGOs waren der Beratungs- bzw. Betreuungsrahmen maßgeblich und die hier geltenden Datenschutzbestimmungen, die in Coronazeiten dann eine detailliertere Betreuung oft verunmöglichten. What's App beispielsweise durfte von den offiziellen Einrichtungen nicht verwendet werden, da es den Datenschutzvorgaben nicht entspricht. Die Klient/ inn/en konnten aber aufgrund ihrere eigenen technischen Möglichkeiten nicht auf die offizellen Programme der Betreuungseinrichtungen zugreifen. Dies wurde von manchen als ein Grund angegeben, weshalb der Kontakt seitens der Klient/inn/en abgebrochen und kaum wiederaufgebaut werden konnte. Dabei ging es beispielsweise um konkrete Hilfestellungen wie die Übermittlung relevanter Unterlagen für den Erhalt von Lebensmittelpaketen oder Informationen über die Zugänge zu Sozialmärkten.

Innerhalb der afghanischen Communities hatten aber keineswegs alle Kontakte zu Vereinen und manche bestehenden Vereine konnten den zusätzlichen Aufgaben von Informationsbeschaffung oder Beratungsleistungen nicht nachkommen.

Es soll auch nicht verschwiegen werden, dass wie in der Mehrheitsbevölkerung auch nicht alle Befragten jeweils alle Vorschriften lückenlos befolgt haben. Dies lag zum einen an Unklarheiten bezüglich der Maßnahmen, an sprachlichen Hürden und mangelnder Information, aber auch an persönlichen Faktoren. Manche Familien haben versucht, im kleinen Kreis Kontakte aufrechtzuerhalten, einander regelmäßig zu sehen 
Abb. 2.3.2: Informationsquellen und deren Nutzungshäufigkeiten (Herkunft Syrien)

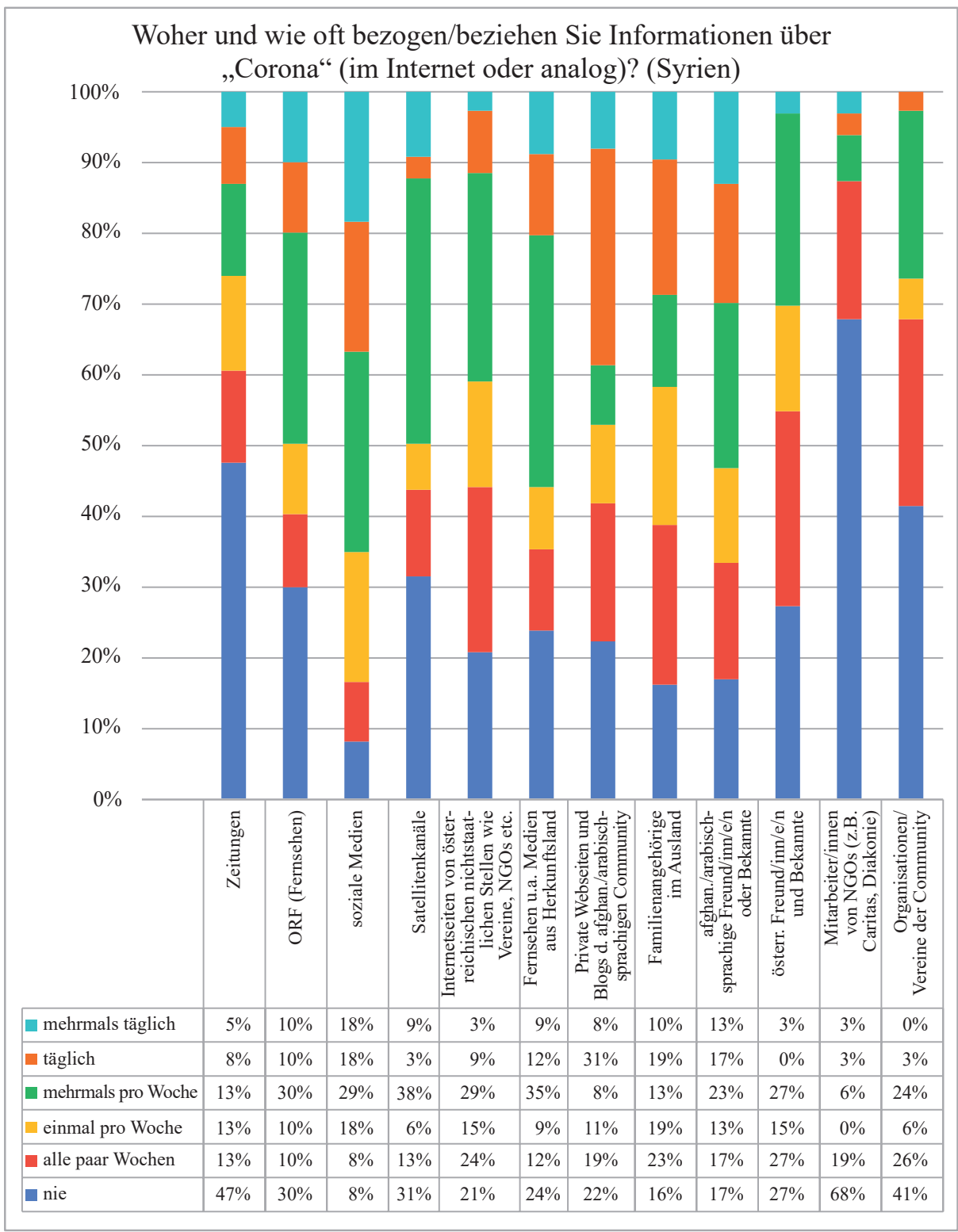

Quelle: eigene Erhebung 2020.

oder zu ermöglichen, dass die Kinder Kontakte untereinander halten konnten oder dass man einander weiterhin materiell und ideell unterstützen konnte. 
Etwas anders als bei den Afghan/inn/en stellt sich die Struktur der Informationsquellen in der syrischen Communities dar (vgl. Abb. 2.3.2). Die höchsten Werte der täglichen oder sogar mehrmals täglichen Nutzung erzielten private Websiten und Blogs der Communities (39\%), Social Media (36\%), arabischsprachige Freund/inn/e/n bzw. Bekannte (30\%) und Familienangehörige im Ausland (29\%) sowie auch der ORF (20\%). Die genannten Informationsquellen wurden von besonders vielen syrischen Befragten auch mehrmals wöchentlich oder zumindest einmal pro Woche zur Beschaffung aktueller Informationen herangezogen. Den höchsten Wert der Nichtnutzung erreichten hier mit 68\% die Mitarbeiter/innen von NGOs, an zweiter Stelle lagen Zeitungen (47\%) und mit $41 \%$ die Vereine der Communities. Am wenigsten bzw. gar nicht genutzt (8\%) wurden Social Media in jeglicher Form sowie mit 16 bzw. 17\% Familienangehörige im Ausland sowie arabischsprachige Freund/inn/e/n in Österreich. Deutlich häufiger als im Fall der afghanischen Communities haben aus Syrien stammende Personen die Angebote der Organisationen und Vereine in Anspruch genommen. 24\% haben sich über diese mehrmals pro Woche zu COVID-19 informiert, 6\% zumindest einmal pro Woche und 26\% unregelmäßig, aber alle paar Wochen. 27\% haben alle paar Wochen auch Informationen von österreichischen Freund/inn/en und Bekannten eingeholt. In derselben Häufigkeit taten dies 24\% über die Internetseiten von österreichischen nichtstaatlichen Stellen.

Wie bereits erwähnt gab es eine Reihe von Initiativen in den arabischen Communities, die sich auf die Übersetzungen von österreichischen Medien fokussiert haben. Ein ägyptischer Privatmann hat täglich die Informationen und Maßnahmen der Regierung übersetzt, wie auch der Nationalratsabgeordnete Omar al-Rawi. Die verschiedenen Ärzt/inn/e/ninitiativen, die Hotlines gründeten, oder etablierte Hilfsvereine wie „Amal“, die bereits eine differenzierte Struktur haben und mehrere hundert Klient/inn/en betreuten, konnten vor allem über soziale Medien relativ rasch unterschiedliche Informationskanäle anbieten. Diese Initiativen waren entweder aus den Communities selbst entstanden oder sie sind gemeinsam mit oder durch Unterstützung von Angehörigen der jeweiligen Community (aus Syrien stammende palästinensische Community, Christ/ inn/en aus Syrien und dem Irak etc.) entstanden. So konnten relativ rasch und auch effizient unterschiedliche Informationsschienen aufgebaut werden. Diese Angebote sind dann sukzessive auch auf direkte Unterstützungsleistungen wie beispielsweise Beschaffung von Medikamenten in Apotheken und die Organisation von Einkäufen ausgedehnt worden.

\subsection{Der Zusammenhang von Informationsbeschaffung und Bildungsstand}

Im Fragenkomplex 2 zur Informationsbeschaffung zeigten sich markante Bildungsunterschiede hinsichtlich des Zugangs zu verschiedenen Informationskanälen und 
-medien. So steigt die Frequenz der Einholung von Informationen über COVID-19 aus den Printmedien mit dem Level des Bildungsabschlusses deutlich an. Von jenen mit sekundärer Schulbildung haben sich mehr als 18\% täglich informiert, ein Wert der sogar höher ist als bei den Universitätsabsolvent/inn/en mit 11,5\%. Zwar sind die Anteile jener, die sich gar nicht für auf Corona bezogene Informationen interessierten, durchwegs relativ hoch, aber mit Abstand am höchsten in der Subkategorie derjenigen mit Primarschulbildung $(68,2 \%)$ und sogar etwas höher bei Uni-Absolvent/inn/en $(42,3 \%)$ als bei Befragten ohne Bildungsabschluss (40\%). Nur alle paar Wochen haben sich $50 \%$ der Befragten ohne Schulabschluss aus Zeitungen informiert, gegenüber jeweils rund einem Fünftel bei den Geflüchteten mit sekundärem oder tertiärem Abschluss. Einmal pro Woche haben sich vor allem Personen mit tertiärer Bildung (15,4\%) über Zeitungen informiert.

Obwohl unser Sample eine nach Alter, Geschlecht und Bildungsstand breit gefasst bzw. divers ist, können wir anhand der begrenzten Anzahl an Respondent/inn/en noch nicht von repräsentativen Informationen sprechen. Die Resultate gewähren vielmehr einen Einblick in Dynamiken und Tendenzen, die die Orientierungen der Befragten während des ersten Lockdowns nachzeichnen.

Die Nutzung des ORF stieg mit dem Bildungslevel stark an. Mehrmals täglich wurden ORF-Nachrichten von 20,8\% der Befragten mit Tertiärbildung, aber nur von $4,5 \%$ jener mit Primärbildung genutzt. Letztere haben aber immerhin zu $18 \%$ täglich den ORF gesehen, gegenüber einem Zehntel der Sekundärgebildeten und 12,5\% der Akademiker/innen. Befragte ohne Bildungsabschlüsse haben ORF-Nachrichten entweder gar nicht oder bestenfalls alle paar Wochen rezipiert, eine Tendenz, die sich ähnlich auch bei den Geflüchteten mit primären Abschlüssen zeigt. Eine wesentliche Erkenntnis aus der Befragung war, dass vor allem die ORF-Nachrichten aufgrund von Übersetzungen, die von Privatpersonen initiiert wurden, wichtige Bezugsquelle waren. Auf diesen Punkt ist in der Befragung noch nicht Bezug genommen worden und auch nicht darauf, ob spezielle Informationsquellen im ORF (wie Nachrichten in einfacher Sprache, Teletext) konsumiert wurden.

Die Nutzung von Social Media war in allen Bildungsgruppen sehr stark ausgeprägt. Zwar haben 37,5\% der Personen ohne Bildungsabschluss auch keine sozialen Medien genutzt, aber immerhin jeweils $25 \%$ taten dies täglich oder sogar mehrmals täglich. Anteile von jeweils fast $22 \%$ auch bei den Akademiker/inn/en informierten sich täglich oder mehrmals täglich aus diesen digitalen Quellen. Geflüchtete mit Primarschulbildung haben zu fast 30\% mehrmals die Woche Informationen über Social Media eingeholt, in derselben Frequenz machten dies fast 17\% der Sekundarschulabsolvent/ inn/en sowie 25\% der Akademiker/innen. Die Nutzung einmal pro Woche verteilt sich relativ gleichmäßig über die Bildungsgruppen von der Primarschule bis hinauf zu den tertiären Abschlüssen. Die seltene Nutzung (alle paar Wochen) wurde vor allem von fast einem Fünftel der Absolvent/inn/en sekundärer Abschlüsse erwähnt, bei den anderen Gruppen waren diese Anteile gering. 
Satellitenkanäle wurden am wenigsten von den Personen mit primären sowie sekundären Abschlüssen genutzt (zu je zwei Drittel gar nicht). Die stärkste Nutzung fand sich unter Akademiker/inne/n, bei denen 36,4\% mehrmals wöchentlich Informationen einholten, sowie unter den Befragten ohne Schulabschluss (33,3\%). Alle paar Wochen haben sich mehr als ein Fünftel der Befragten mit primären Abschlüssen und rund 18\% der Sekundarschulabsolvent/inn/en Satellitenkanäle für die auf Corona bezogenen Informationen angesehen. Unter den Akademiker/inne/n belief sich der Anteil auf nur 9\%.

Für die Internetseiten von österreichischen NGOs haben sich in erster Linie Befragte mit höheren Bildungslevels interessiert. 83\% der Respondent/inn/en ohne Bildungsabschluss haben diese gar nicht besucht, dafür 21,7\% der Hochschulabsolvent/ inn/en mehrmals pro Woche und 8,7\% sogar täglich. Mehr als die Hälfte der Letztgenannten holten die Pandemieinformationen einmal pro Woche oder zumindest alle paar Wochen über die Websites der NGOs ein.

Fernsehen und Medien aus dem Herkunftsland wurden etwas häufiger von Personen mit geringerer Schulbildung konsumiert. In der Kategorie ohne Schulabschluss von der Hälfte der Befragten täglich und von einem Drittel alle paar Wochen, von rund $17 \%$ mehrmals wöchentlich. Zwar war auch bei den Geflüchteten mit Tertiärbildung die Nutzung der Medien aus der Heimat im Durchschnitt recht hoch, fand jedoch in geringeren Frequenzen statt: Rund 18\% nutzten diese täglich oder mehrmals pro Tag, 22,7\% mehrmals pro Woche, dafür aber fast 32\% gar nicht. In den Bildungskategorien Primarsowie Sekundarschule waren jeweils die Häufigkeiten der Kategorie ,gar nicht“ am höchsten, darüber hinaus auch die Nutzung nur alle paar Wochen. Websites der eigenen Communities wurden von Geflüchteten mit tertiären Bildungslevels zu fast ähnlich hohen Anteilen (20\%) täglich genutzt wie von Befragten ohne Schulbildung oder mit primärer Bildung (jeweils 25\%). Auch hinsichtlich der Nutzung nur alle paar Wochen zeigten sich eher geringe Unterschiede nach dem Bildungslevel (jeweils ein Viertel der Hochschulabsolvent/inn/en sowie ein gleicher Anteil bei den Respondent/inn/en ohne Schulabschluss und auch bei jenen mit Primarschulbildung).

Familienangehörige im Ausland haben vor allem weniger Gebildete häufig zwecks der Einholung der auf Corona bezogenen Informationen kontaktiert. Jeweils ein Fünftel der Geflüchteten ohne Schulabschluss sowie jener mit Primarschulbildung, aber nur 5,3\% der tertiär Gebildeten taten dies täglich, erstgenannte zu $60 \%$ mehrmals wöchentlich (gegenüber nur 5,3\% der Universitätsabsolvent/inn/en). Zu hohen Anteilen wurden im Ausland lebende Familienmitglieder alle paar Wochen als Informationsquelle zu Corona kontaktiert. Jeweils $40 \%$ der Respondent/inn/en mit primärer bzw. sekundärer Bildung taten dies sowie fast 32\% der Akademiker/inn/en. Im Fragebogen wurde nicht näher spezifiziert, ob es sich um Familienangehörige im Herkunftsland handelte, oder um Personen, die in anderen Ländern um Asyl angesucht hatten oder um Familienmitglieder, die sich noch in Transitländern aufhielten.

Freund/inn/e/n aus den eigenen ethnischen Communities spielten für die tägliche Informationsbeschaffung in erster Linie bei einem Fünftel der Geflüchteten ohne Schul- 
abschluss eine Rolle, aber nur bei 5,6\% der tertiär Gebildeten. Auch bei den selteneren Nutzungen (einmal pro Woche bzw. alle paar Wochen) dominierten die Personen ohne Schulabschlüsse, während ein Drittel der tertiär Gebildeten und sogar 41,7\% der Sekundarabsolvent/inn/en Freund/inn/e/n aus den eigenen Communities gar nicht um Informationen zu Corona kontaktierten. Alle paar Wochen haben vor allem Befragte mit sekundären Abschlüssen (29,2\%) sowie Universitätsabsolvent/inn/en (27,8\%) ihre Freund/inn/e/n oder Bekannten zu Corona befragt.

Österreichische Freund/inn/e/n spielten ebenfalls primär bei den Befragten auf den mittleren und höheren Bildungsstufen eine wichtigere Rolle als Informationsquellen. Hierbei war wohl der Faktor der besseren Deutschkompetenz wichtig. Informationen durch Freund/inn/e/n/Bekannte aus Österreich haben nur 22,7\% der Geflüchteten in der höchsten Bildungsstufe gar nicht eingeholt, dafür aber $60 \%$ der Personen ohne jeglichen Schulabschluss. Mehrmals pro Woche wurden österreichische Freund/inn/e/n/Bekannte von einem Viertel der Sekundarschulabsolvent/inn/en und einem Fünftel der Primarschulgebildeten kontaktiert, weiters von 13,6\% der tertiär gebildeten Befragten.

Die Kontaktierung von NGO-Mitarbeiter/inne/n war in der höchsten Bildungskategorie am häufigsten. Jeweils 5\% der Geflüchteten mit tertiärer Bildung holten mehrmals täglich, täglich oder mehrmals pro Woche Informationen über diese Quelle ein, 25\% zumindest alle paar Wochen. Die geringste Rolle spielten NGO-Mitarbeiter/ innen bei Geflüchteten mit Sekundarbildung, aber auch bei drei Vierteln der Befragten mit Primarschulbildung sowie jenen ohne Bildungsabschlüsse. Die Gründe hierfür sind noch nicht eruiert und müssten in einer Nachfolgebefragung genauer thematisiert werden. Gründe, die indirekt aus den Expert/inn/eninterviews hervorgingen, könnten in der Notwendigkeit der Umstellung von direkten persönlichen Kontakten zu verschriftlichten Informationen sein bzw. der Wegfall der Face-to-Face-Kontakte während des ersten Lockdowns.

Organisationen der eigenen Communities wurden in sehr unterschiedlichem Ausmaß zum Zweck der Coronainformation herangezogen. Die Häufigkeiten sind vor allem in den Kategorien mehrmals pro Woche sowie alle paar Wochen am höchsten. Mehrmals wöchentlich holten vor allem Geflüchtete mit primärer Schulbildung $(23,8 \%)$ sowie jene ohne Schulabschluss (20\%) Informationen ein. Akademiker/innen nur zu 9,5\%. Von letzteren kontaktierten aber 42,9\% Vereine der Community alle paar Wochen und jeweils rund 23\% der Sekundarschul- und Primarschulgebildeten taten dies ebenfalls. Unter den Befragten ohne Schulabschluss lag der Anteil bei 20\%. 


\subsection{Vertrauen in Informationsquellen zu COVID-19}

Abb. 2.4: Ausmaß des Vertrauens in die verschiedenen Informationsquellen (gesamt)

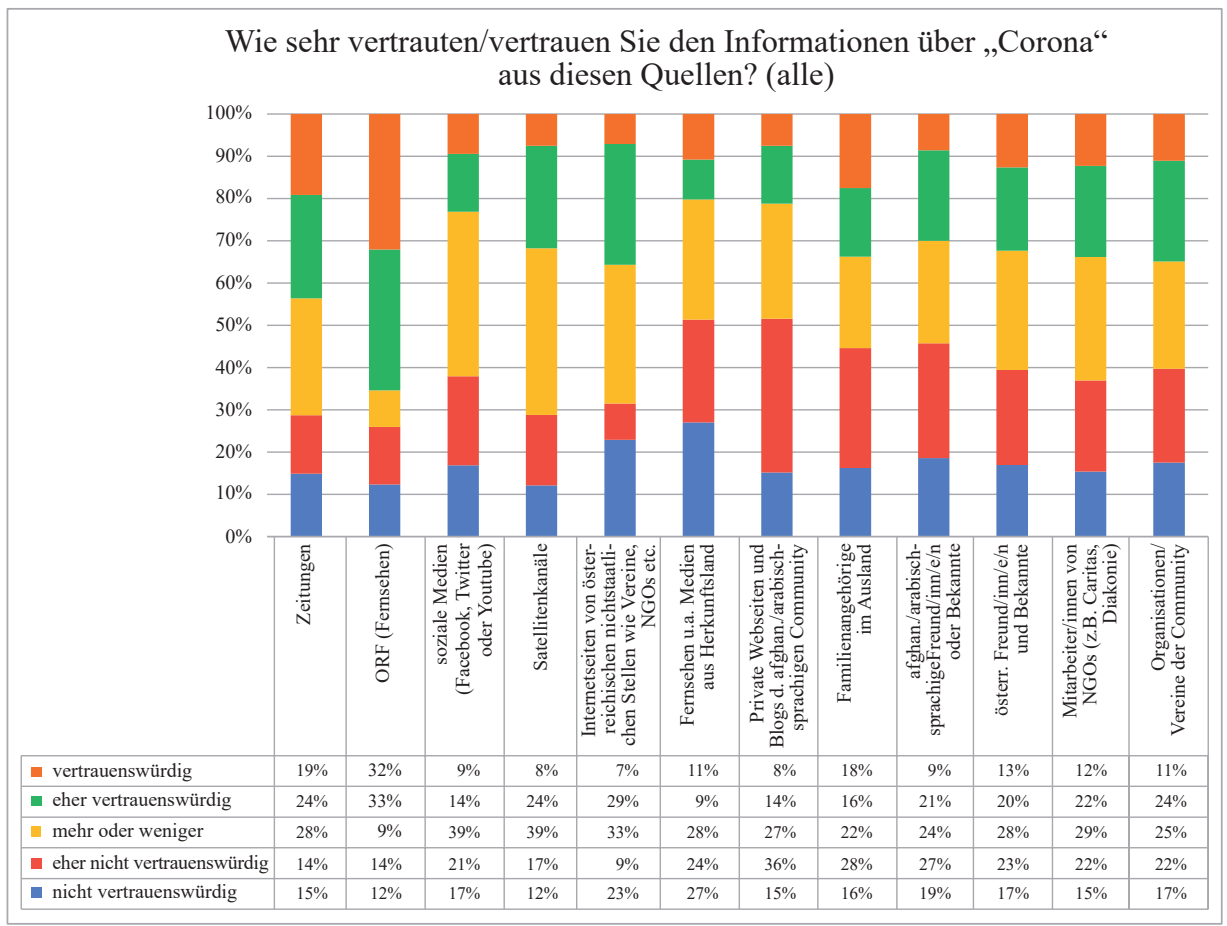

Quelle: eigene Erhebung 2020.

Hinsichtlich der den aufgezählten Informationsquellen zugeschriebenen Vertrauenswürdigkeit zeigt sich bezogen auf das gesamte Sample ein differenziertes Bild (vgl. Abb. 2.4). Die höchsten Werte an Nichtvertrauenswürdigkeit entfallen auf TV bzw. Medien der Herkunftsländer (27\%) sowie die Internetseiten von österreichischen nichtstaatlichen Stellen (23\%), aber auch arabischsprachige Freund/inn/e/n (19\%), Vereine der Communities (17\%) und Social Media (17\%) erreichen hier analoge Werte. Den Spitzenwert an Vertrauenswürdigkeit erreicht mit 32\% der ORF, darauf folgen Printmedien (19\%) und Familienangehörige im Ausland (18\%). Den ORF klassifizierten weitere 33\% als eher vertrauenswürdig, jeweils 24\% schrieben dies Zeitungen und Satellitenkanälen zu und 29\% den Internetseiten österreichischer nichtstaatlicher Organisationen. 51\% klassifizierten private Internetseiten und Blogs der arabischsprachigen Communities als wenig bzw. gar nicht vertrauenswürdig. Mit dem gleichen Prozentsatz wurden Fernsehen und Medien aus dem Herkunftsland als wenig bzw. gar nicht vertrau- 
enswürdig bewertet sowie von $48 \%$ der Befragten Freund/inn/e/n aus der eigenen Community. 44\% schrieben sogar Familienangehörigen, welche im Ausland lebten, wenig bis keine Vertrauenswürdigkeit hinsichtlich ihrer COVID-19-Informationen zu.

Auch in den qualitativen Interviews mit Geflüchteten aus Syrien und mit Expert/ inn/en wurde betont, dass eine Reihe von Einzelinitiativen, die über soziale Medien spezifische Informationen gepostet haben, vorhanden waren. Diesen privaten Seiten in den sozialen Medien hat man weniger Vertrauen entgegengebracht, manchmal auch weil politische Überlegungen in der Informationsverbreitung damit verbunden waren. Eine Expertin meinte auch, dass nicht alle Initiativen in den sozialen Medien adäquat waren, da sie in dem einen oder anderen Bereich Persönlichkeitsrechte der Betroffenen bzw. die Datenschutzvorgaben nicht ernst genug nahmen und so auch vertrauliche Informationen über Patient/inn/en im Internet aufgetaucht wären und an Hand solcher Beispiele bestimmte medizinische oder verwandte Fragen diskutiert wurden.

Abb. 2.4.1: Ausmaß des Vertrauens in die verschiedenen Informationsquellen (Herkunft Afghanistan)

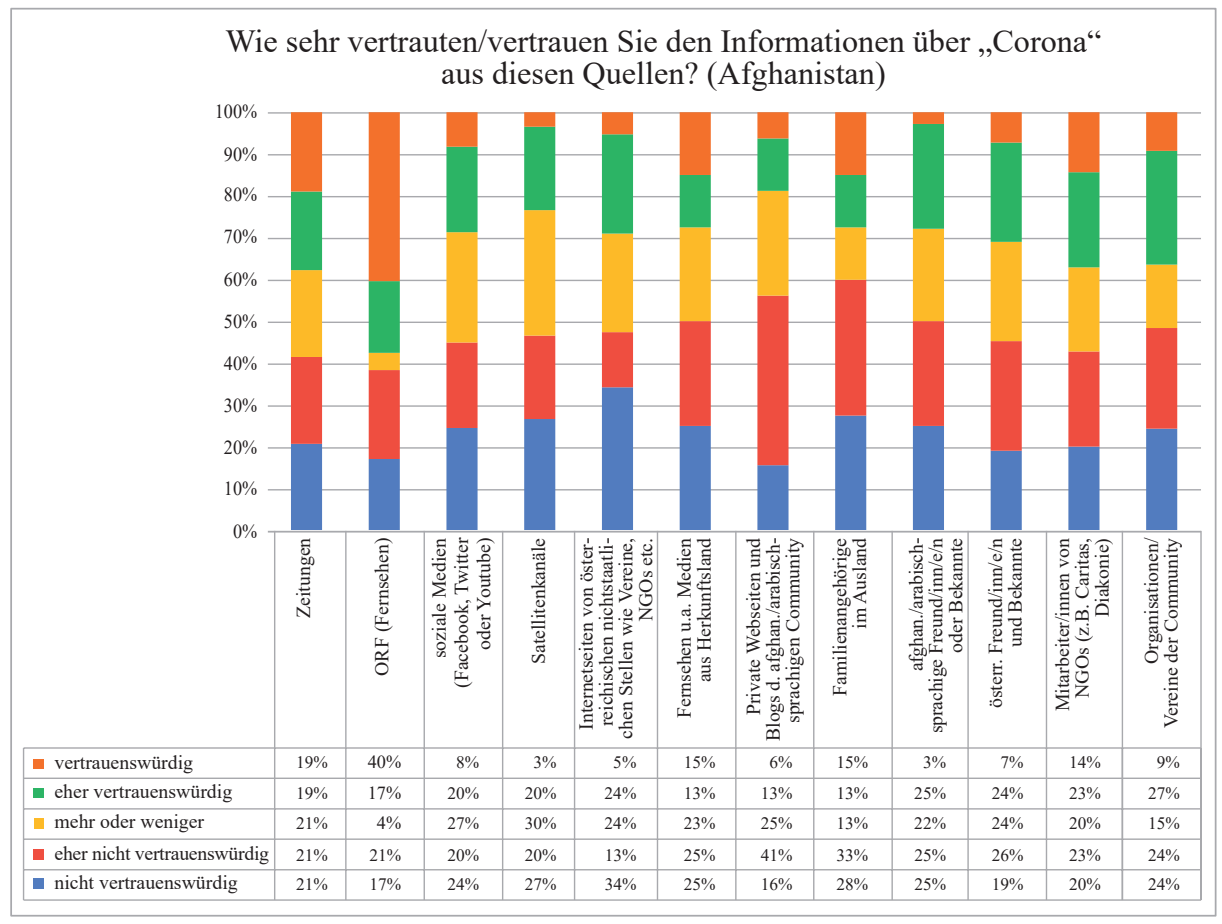

Quelle: eigene Erhebung 2020. 
Afghanische Befragte (vgl. Abb. 2.4.1) vertrauten im Ausland lebenden Familienmitgliedern in Bezug auf deren Coronakompetenz am wenigsten (61\% gaben die Bewertung wenig bis gar nicht vertrauenswürdig ab), auch private Webseiten und Blogs der afghanischen Communities wurden zu 57\% mit derselben negativen Einschätzung belegt. Relativ gleich eingeschätzt wurden diesbezüglich afghanische Freund/inn/e/n oder Bekannte sowie Medien aus dem Herkunftsland. Etwas bessere Werte hinsichtlich ihrer Vertrauenswürdigkeit erzielten österreichische Freund/inn/e/n bzw. Bekannte, Mitarbeiter/innen von NGOs, aber auch Informationen seitens der Vereine der eigenen Community. Den höchsten Wert an Vertrauenswürdigkeit verbuchte der ORF, dessen Informationen von 57\% als (eher) vertrauenswürdig bewertet wurden sowie Satellitenkanäle (53\%). Mit deutlichem Abstand folgten Printmedien (38\%), Mitarbeiter/innen von NGOs (37\%), die Vereine der Communities (36\%) und österreichische Freund/ inn/e/n (31\%). Die Vertrauenswürdigkeit der Informationen wurde auch in den qualitativen Interviews sehr unterschiedlich beurteilt.

Abb. 2.4.2: Ausmaß des Vertrauens in die verschiedenen Informationsquellen (Herkunft Syrien)

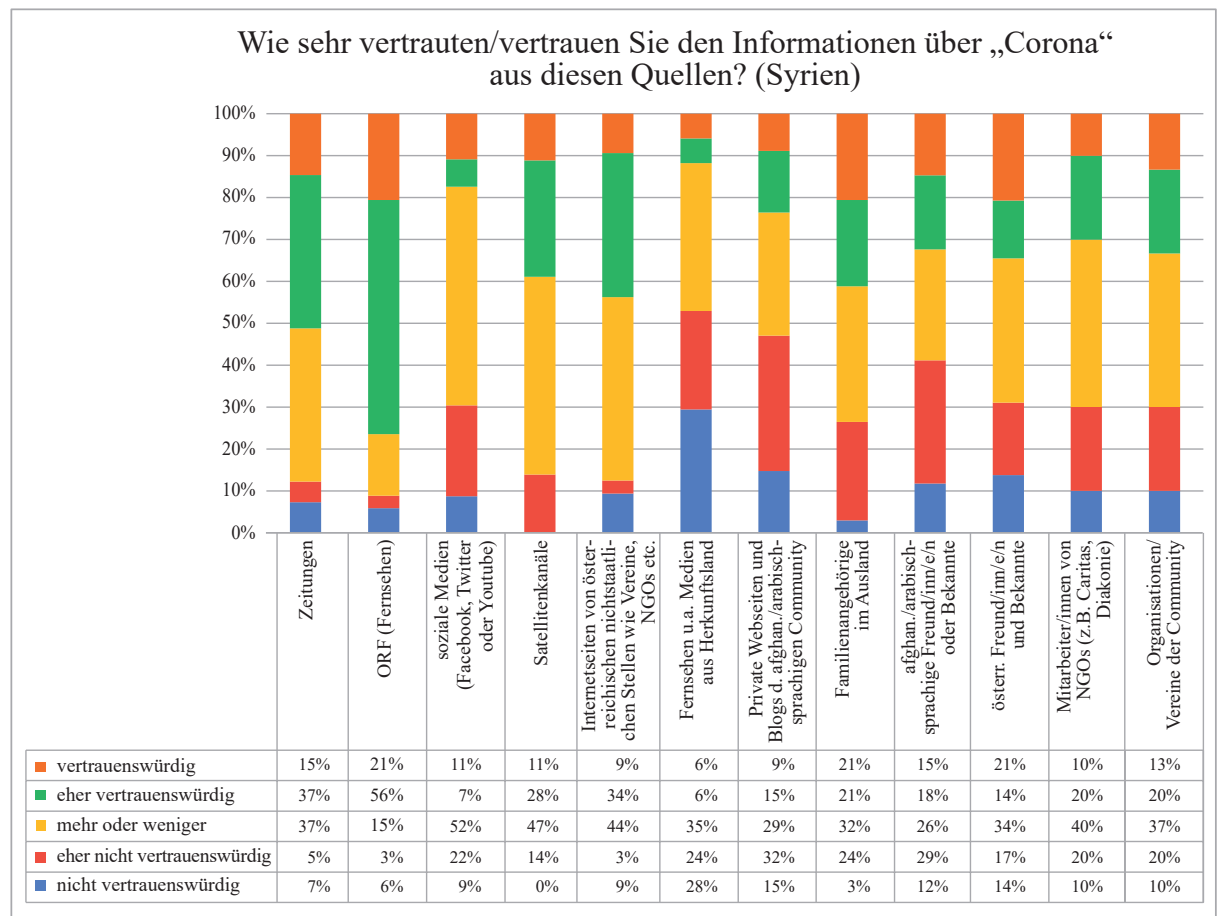

Quelle: eigene Erhebung 2020. 
Die von den NGOs bereitgestellten Informationen wurden weitgehend als zuverlässig eingestuft, wenngleich es schwierig ist, dies aus der Sicht der NGOs wirklich $\mathrm{zu}$ beurteilen. Keinesfalls immer waren die online verfügbaren Informationsquellen als zuverlässig einzuordnen. Dies galt vor allem auch für muttersprachliche Medien, deren Quellen nicht explizit dargestellt wurden. Einige Respondent/inn/en berichteten, dass sie den offiziellen österreichischen Medien das meiste Vertrauen schenkten. Jedoch hatten viele am Anfang der Pandemie erfahren, dass offizielle bzw. übersetzte Informationen nicht vollständig und daher keineswegs immer verlässlich waren.

Im Falle der syrischen Communities (vgl. Abb. 2.4.2) wurden dem ORF (92\%), Zeitungen $(89 \%)$, den Internetseiten von österreichischen nichtstaatlichen Organisationen (87\%) und Satellitenkanälen (86\%) höchste Levels an Vertrauenswürdigkeit entgegengebracht. Die von diesen Quellen zur Verfügung gestellten Informationen wurden als vertrauenswürdig, eher vertrauenswürdig oder mehr oder weniger vertrauenswürdig bewertet. Auf die täglichen Übersetzungsleistungen von Privatpersonen wurde bereits oben näher verwiesen. Soziale Medien wurden ein wenig kritischer gesehen und ernteten in Bezug auf deren Vertrauenswürdigkeit einen Wert von $70 \%$. Von 29\% wurden Medien aus dem Herkunftsland mit großem Abstand als am wenigsten vertrauenswürdig beurteilt, darauf folgten private Webseiten und Blogs der arabischsprachigen Communities mit 15\%. Zu jeweils relativ gleichen Anteilen wurde österreichischen Freund/inn/e/n, Familienmitgliedern im Ausland, Mitarbeiter/inne/n von NGOs sowie den Vereinen der eigenen Community Vertrauenswürdigkeit in der Informationsbereitstellung zugeschrieben, ein etwas geringeres Maß an Vertrauen brachten die Befragten aus Syrien arabischsprachigen Freund/inn/e/n entgegen.

$\mathrm{Zu}$ bedenken ist in den Ausführungen zu sozialen Medien, ob und wie die Menschen vor allem in den Zeiten des Lockdowns Zugang dazu hatten. Erfahrungen von sozialen Einrichtungen in Deutschland und von Expert/inn/en in Österreich zeigten, dass nicht alle Menschen gleichermaßen den Zugang zum technischen Equipment (Smartphones, Computer) oder zu einem Internetanschluss hatten. Manche Flüchtlingsunterkünfte haben kein allgemein verfügbares WLAN und die Menschen konnten daher im Lockdown weder mit ihren Betreuer/inne/n noch mit ihren Verwandten oder Mitarbeiter/inne/n ausreichend kommunizieren. Dazu kommt noch, dass nicht alle Geflüchteten ausreichend in Arabisch oder Farsi-Dari alphabetisiert sind, noch keine genügenden Deutschkenntnisse aufwiesen und auch deshalb diesen Informationen nicht folgen konnten. Dadurch wurde auch die Kommunikation mit Betreuer/inne/n über beispielsweise rechtlich relevante Schritte (Übersetzungen von Schriftstücken etc.) erschwert. Einige der in der qualitatitiven Erhebung Befragten wiesen darauf hin, dass mündlich verfasste Informationen im Internet daher eine größere Reichweite hatten als schriftliche Übersetzungen oder Erläuterungen. 


\subsection{Bildungsniveau und Vertrauen in Informationen}

Eine wichtige Frage bezog sich darauf, inwieweit die Geflüchteten den Informationen auch Vertrauen schenkten. Hierbei zeigten sich deutliche Unterschiede nach Bildungsabschlüssen. Österreichischen Printmedien wurde der höchste Wert an Nichtvertrauenswürdigkeit seitens der Befragten ohne Bildungsabschluss $(37,5 \%)$ zugeordnet, während Befragte mit tertiärem Bildungsabschluss nur zu 10,7\% und solche mit Sekundärbildung zu 9\% dieser Meinung waren. Bereits in der Gruppe mit Primarschulbildung sprang der entsprechende Prozentwert auf 21,7\%. Hingegen war das Level an Vertrauenswürdigkeit mit Werten um die 17\% bzw. 18\% bei allen Bildungsgruppen ab der Primarschulbildung relativ gleichmäßig hoch, am geringsten jedoch bei Respondent/ inn/en ohne Schulabschluss mit 12,5\%.

Noch deutlicher trat der Konnex zwischen höherem Bildungsabschluss und dem Vertrauen in die Verlässlichkeit der Informationen im Falle der ORF-Nachrichten hervor. Zwei Drittel der Geflüchteten ohne Schulbildung plädierten bei dieser Kategorie von Nachrichten für nicht vertrauenswürdig, 41,2\% der Befragten mit Primarschulabschluss für eher nicht vertrauenswürdig. 50\% der tertiär Gebildeten sowie fast ein Drittel der sekundär Gebildeten hielten den ORF hingegen für eher vertrauenswürdig. In der Kategorie ,vertrauenswürdig“ zeigte sich eine relativ gleiche Verteilung über alle Bildungslevels hinweg, am höchsten waren die Anteile jener, welche den ORF als vertrauenswürdig einschätzten, bei den Sekundarschulabsolvent/inn/en (35,3\%) sowie den Geflüchteten ohne Schulabschlüsse (33,3\%), bei etwa 30\% lag die Zustimmung aber auch in den beiden anderen Bildungskategorien.

Social Media wurden von 29\% der Sekundarschulabsolvent/inn/en sowie 23,3\% jener mit tertiärer Bildung als eher nicht vertrauenswürdig klassifiziert. Die höchsten Ratings an Nichtvertrauenswürdigkeit erhielten Social Media mit jeweils rund einem Drittel von den Befragten ohne Schulabschluss sowie jenen mit Primärschulbildung. Als mehr oder weniger vertrauenswürdig schätzten vor allem jene aus der niedrigsten Bildungsgruppe (66,7\%) sowie die Uni-Absolvent/inn/en (46,7\%) die sozialen Medien ein.

Satellitenkanäle wurden am ehesten von tertiär Gebildeten $(15,8 \%)$ als vertrauenswürdig klassifiziert, ein exakt gleicher Prozentsatz schätzte diese Quellen aber auch als nicht vertrauenswürdig ein. Diese Meinung wurde auch von einem Fünftel der Befragten mit Primarschulbildung abgegeben. Von den letztgenannten sowie den Geflüchteten mit Primarschule gab jeweils rund ein Drittel der Befragten die Bewertung als eher vertrauenswürdig ab. Mit 26,3\% war hier der Anteil der höchsten Bildungskategorie niedriger. Generell stellt sich dabei auch die Frage der Verfügbarkeit von Satellitenkanälen, die im Fragebogen nicht erhoben wurde.

Den Internetseiten von NGOs und Vereinen in Österreich wurde von fast 39\% der Primarschulabgänger/innen das Etikett als nicht vertrauenswürdig zugedacht. Mit steigendem Bildungsabschluss stieg hier die Einschätzung hinsichtlich der Vertrauens- 
würdigkeit deutlich an. 13,6\% der Uni-Absolvent/inn/en und 7,7\% der Sekundarschulgebildeten hielten die Internetseiten der NGOS für vertrauenswürdig.

Den Medien aus dem Herkunftsland sprachen eher die Geflüchteten mit mittleren Bildungslevels eine höhere Vertrauenswürdigkeit zu. 15,8\% der Primarschulabsolvent/ inn/en und 12\% der Absolvent/inn/en sekundärer Abschlüsse hielten TV und Medien aus den Herkunftskontexten für vertrauenswürdig, dafür nur 8,7\% der tertiär Gebildeten. Die meisten Befragten plädierten hier für eher vertrauenswürdig bzw. mehr oder weniger vertrauenswürdig $(60 \%$ in der Kategorie ohne Schulabschluss, aber auch rund $39 \%$ der tertiär Gebildeten und 60\% der Befragten mit sekundären Abschlüssen). Auch hier wurde in der Befragung nicht genau spezifiziert. Mittlerweile ist eine Vielzahl von transnational organisierten SAT-TV oder anderen Medien, die von herrschenden politischen Kräften wie auch Gegner/inne/n produziert werden, verfügbar. Auf welche davon die Befragten Bezug genommen haben, geht aus den Befragungen nicht hervor.

Private Webseiten und Blogs der eigenen Communities wurden vor allem von Geflüchteten mit tertiären Abschlüssen als nicht oder eher nicht vertrauenswürdig klassifiziert (26\%). Von den Primarschulgebildeten meinten hingegen 16,7\% diese Quelle sei vertrauenswürdig. Befragte ohne Schulabschluss gaben zu zwei Dritteln eine Beurteilung als eher oder mehr oder weniger vertrauenswürdig ab.

Hinsichtlich der Vertrauenswürdigkeit von Familienangehörigen im Ausland bezüglich der Informationen zu COVID-19 waren die Resultate dispers. Am meisten an Vertrauenswürdigkeit wurde ihnen von Befragten mit sekundären Bildungsabschlüssen zugesprochen (32\%), andererseits plädierte ein Fünftel in derselben Bildungskategorie für keine Vertrauenswürdigkeit und dasselbe galt für 21\% in der Gruppe mit primärem Schulabschluss. Mehr als ein Drittel der höchsten Bildungsgruppe sprachen Familienangehörigen im Ausland zumindest mehr oder weniger Vertrauenswürdigkeit zu. Die Einschätzungen als eher nicht vertrauenswürdig waren jedoch quer über alle Bildungsgruppen hoch: mehr als $26 \%$ bei tertiär und primär Gebildeten and $32 \%$ in der Kategorie mit sekundärem Bildungslevel. Aus den qualitativen Interviews ging hervor, dass Betroffene immer wieder von Meidungsstrategien in der Kommunikation berichteten und über den faktischen gesundheitlichen Zustand oder die jeweilige Situation im Herkunftsland Narrative entwickelten, die nicht unbedingt der Realität entsprachen. Um einander gegenseitig nicht allzu sehr $\mathrm{zu}$ belasten, wurden Informationen oft verschwiegen - dies oft im Bewusstsein beider Seiten.

Die Freund/inn/e/n aus den eigenen Communities rangierten bei einem Viertel der Geflüchteten mit Tertiärabschlüssen in der Kategorie nicht vertrauenswürdig, mit rund $21 \%$ war diese Bewertung aber auch bei Primarschulabsolvent/inn/en relativ hoch. Am höchsten in Bezug auf die Vertrauenswürdigkeit war der Wert dieser Informationsquellen unter Befragten mit Sekundarabschlüssen, von denen 13,6\% ihre Freund/ inn/e/n als vertrauenswürdige Informationsquelle klassifizierten. Als eher nicht vertrauenswürdig galten sie bei $37 \%$ der Befragten mit primärer Bildung und bei $29 \%$ jener mit tertiären Abschlüssen. 
Die Ratings an Nichtvertrauenswürdigkeit gegenüber den Informationen österreichischer Freund/inn/e/n und Bekannter waren quer über die Bildungskategorien relativ gleich hoch. Mit rund 19\% am höchsten in der Kategorie tertiärer Bildung und am niedrigsten mit 16,7\% bei jenen ohne abgeschlossene Schullaufbahn. Dafür erhielten Österreicher/innen von letzteren in keinem Fall die Einschätzung als vertrauenswürdig, was allerdings bei $17,7 \%$ der Absolvent/inn/en primärer Abschlüsse sowie bei $14,3 \%$ der Personen mit tertiären Abschlüssen der Fall war.

Mitarbeiter/innen österreichischer NGOs wurden von 25\% der Befragten ohne Schulbildung sowie 16,7\% jener mit primären Abschlüssen als vertrauenswürdig in Bezug auf ihre Corona-Informationen klassifiziert. Mit 13,6\% war dieser Anteil bei den Geflüchteten mit Tertiärabschlüssen etwas geringer. Letztere beurteilten die NGO-Mitarbeiter/innen aber zu einem exakt gleichen Anteil als nicht vertrauenswürdig. Dies taten zu beträchtlich höheren Anteilen die Befragten ohne Abschlüsse (25\%) sowie auch jene mit primären Bildungsabschlüssen (22,2\%).

Bemerkenswertes Faktum ist, dass den Vereinen und Organisationen der Communities von keinem Befragten eine volle Vertrauenswürdigkeit zugesprochen wurde. Am ehesten vertrauenswürdig fanden diese Informationsquelle die Geflüchteten ohne Schulabschluss (40\%), ein Drittel jener mit primären Abschlüssen und mehr als 19\% in der höchsten Bildungskategorie. Hingegen sprach sich ein Fünftel der Personen im Sample ohne Bildungsabschluss für nicht vertrauenswürdig aus. Dieselbe Einschätzung äußerten auch 19\% jener mit Uni-Abschlüssen sowie jeweils 16,7\% der Geflüchteten mit primären oder sekundären Abschlüssen.

Die obigen Analysen dokumentieren, dass ein hoher Grad an Auseinandersetzung mit und über (Neue) Medien und weiteren Informationskanälen vorliegt, dass sehr diverse Einschätzungen darüber existieren und diese vor allem mit dem Bildungsniveau korrelieren. Des Weiteren zeigt dies die Beschäftigung der Befragten mit sehr diversen Informationskanälen, die vielleicht bei Menschen ohne den Hintergrund der Flucht gar nicht notwendig ist und denen es klarer ist, wie man sich bestimmte Informationen beschaffen kann.

\subsection{Umsetzung der Regierungsmaßnahmen}

Durchwegs sehr hoch war laut den Antworten der Online-Befragung das Ausmaß, in dem die Geflüchteten die von der Regierung beschlossenen Maßnahmen befolgten und umsetzen konnten (vgl. Abb. 2.5). Zu 95\% im Schnitt des gesamten Samples und in höherem Ausmaß (98\%) von den Befragten aus Syrien als von jenen aus Afghanistan (91\%). Aus den qualitativen Interviews gingen auch die Problembereiche hervor, die es manchen Befragten verunmöglichten, die Vorgaben einzuhalten. 
Abb. 2.5: Möglichkeit der Umsetzung der Regierungsmaßnahmen zu Corona (gesamt)

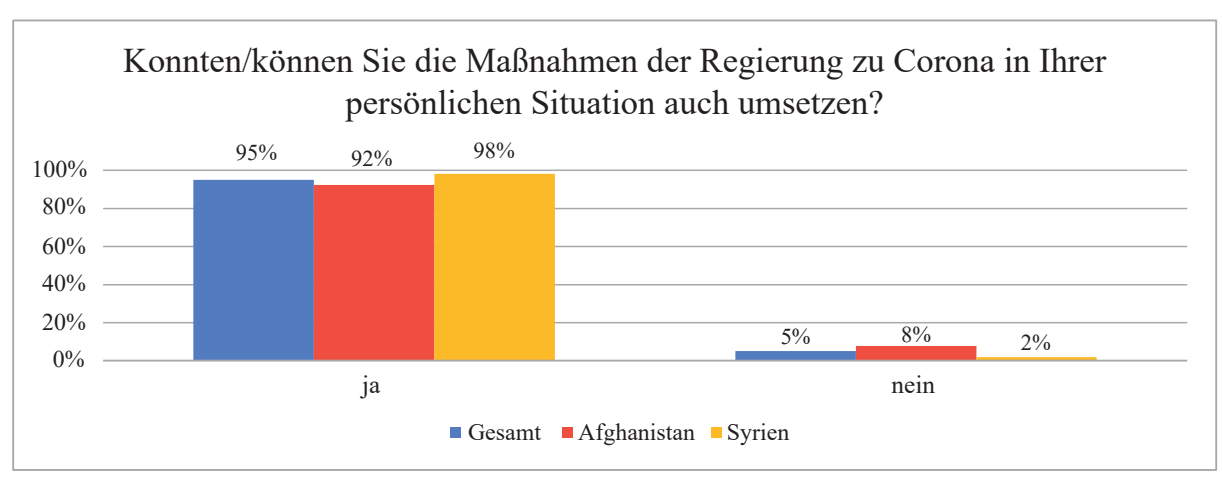

Quelle: eigene Erhebung 2020.

Schwierig war es für Jugendliche, die in Flüchtlingsquartieren oder -heimen untergebracht waren und die aufgrund der räumlichen Beengtheit zwischendurch versuchten hinauszugehen und sich im Freien von der stressigen Wohnsituation zu erholen. Aus den Expert/inn/eninterviews ging hervor, dass Familien mit der Situation besser umgehen konnten. Gerade von jenen Familien, in denen eines oder mehrere Mitglieder eine Vorerkrankung aufwiesen oder in die Gruppe der Risikopatient/inn/en gehörten, wurden die Maßnahmen besonders strikt befolgt. Weiters war das Problembewusstsein für die Situation und die angeordneten Maßnahmen sehr hoch. Ein Informant wies auf die Erfahrungen von Syrer/inne/n hin, die aufgrund der Kriegssituation oft monatelang gezwungen waren, in ihren Häusern zu bleiben. Dieser Informant erklärte jedoch, dass beide Situationen nicht unmittelbar vergleichbar waren und die Gesamtsituation sehr komplex sei. Ein weiterer Umstand für die Nichteinhaltung von Maßnahmen wie Physical Distancing war, dass manche Menschen zu wenig Informationen zur Verfügung hatten, auf die Vermittlung und Aufklärung anderer Personen angewiesen waren oder es einfach am technischen Equipment fehlte.

Auf die Frage warum man bestimmte Maßnahmen nicht umsetzen konnte, wurden auch offene Antworten abgegeben, die darauf verwiesen, dass ,sie nicht immer zu Hause bleiben können“, dass man „nicht lange Zeit zu Hause bleiben kann“, dass man „Familienbedürfnisse zu erfüllen habe“ bzw. „Arbeit suchen musste“ oder dass es gewisse „Lebensbedürfnisse“ gab, denen man nachgehen musste.

Als besonders belastend wurden durchwegs das Zuhausebleiben sowie das Social Distancing empfunden (vgl. Abb. 2.5.1). Als viel weniger problematisch wurden die Einhaltung der hygienischen Maßnahmen oder andere Aspekte eingestuft. Dies spiegelte sich auch in den qualitativen Interviews wider. Für Jugendliche wie auch für ältere Personen war das Social/Physical Distancing aus unterschiedlichen Gründen herausfordernd. Ältere Personen, die nicht im selben Haushalt mit Kindern und Enkelkindern 
Abb. 2.5.1: Welche Maßnahmen waren nicht umsetzbar? (gesamt)

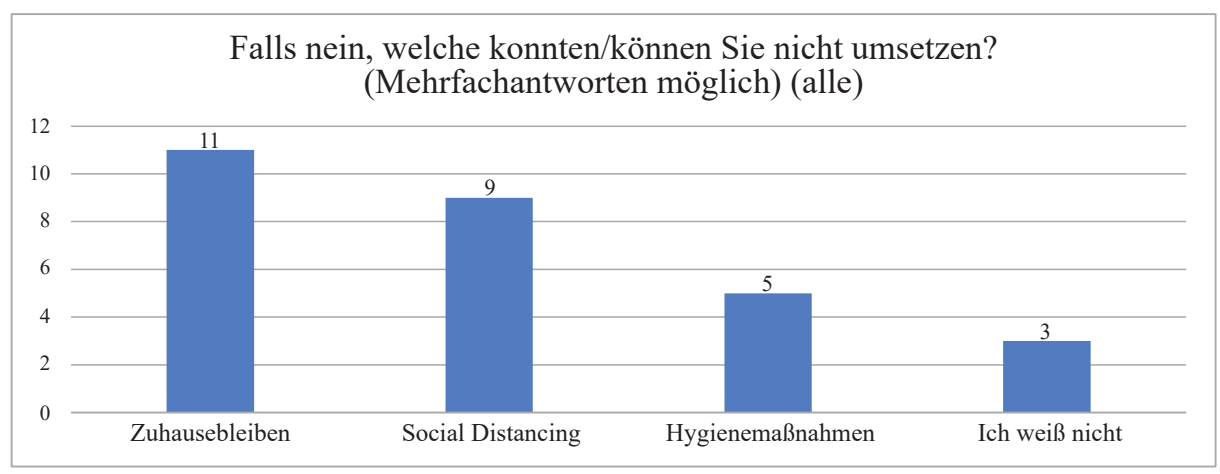

Quelle: eigene Erhebung 2020.

lebten, haben über Monate ihre Enkelkinder nicht gesehen und nur über soziale Medien Kontakt gehalten. Haushalte haben füreinander gesorgt, z.B. gekocht, einander aber nicht besucht. Für Jugendliche, die noch über kein elaboriertes Netzwerk verfügen, sind gerade die Kontakte in der jeweiligen Freundesgruppe besonders wichtig. Sich hierbei auch an die Regeln zu halten, also Distanz zu wahren, war für einige befremdlich. Auch fiel der erste Lockdown in die Zeit des Ramadan, in welchem das Physical Distancing (z.B. beim Fastenbrechen oder am Ende des Ramadan) für manche herausfordernd war. Interessanterweise hat die Politik auf diese Umstände (d.h. große religiöse Feiertage der nichtchristlichen Religionen in Österreich) keinen Bezug genommen, obwohl gerade in den Argumentationen mancher Politiker/innen die christlichen Feiertage einen wesentlichen Punkt in ihren Maßnahmenkatalogen bildeten. 


\section{Sozialkontakte}

\subsection{Häufigkeit von Sozialkontakten}

Abb. 3.1: Häufigkeit der Sozialkontakte im Lockdown nach Personenkreisen (gesamt)

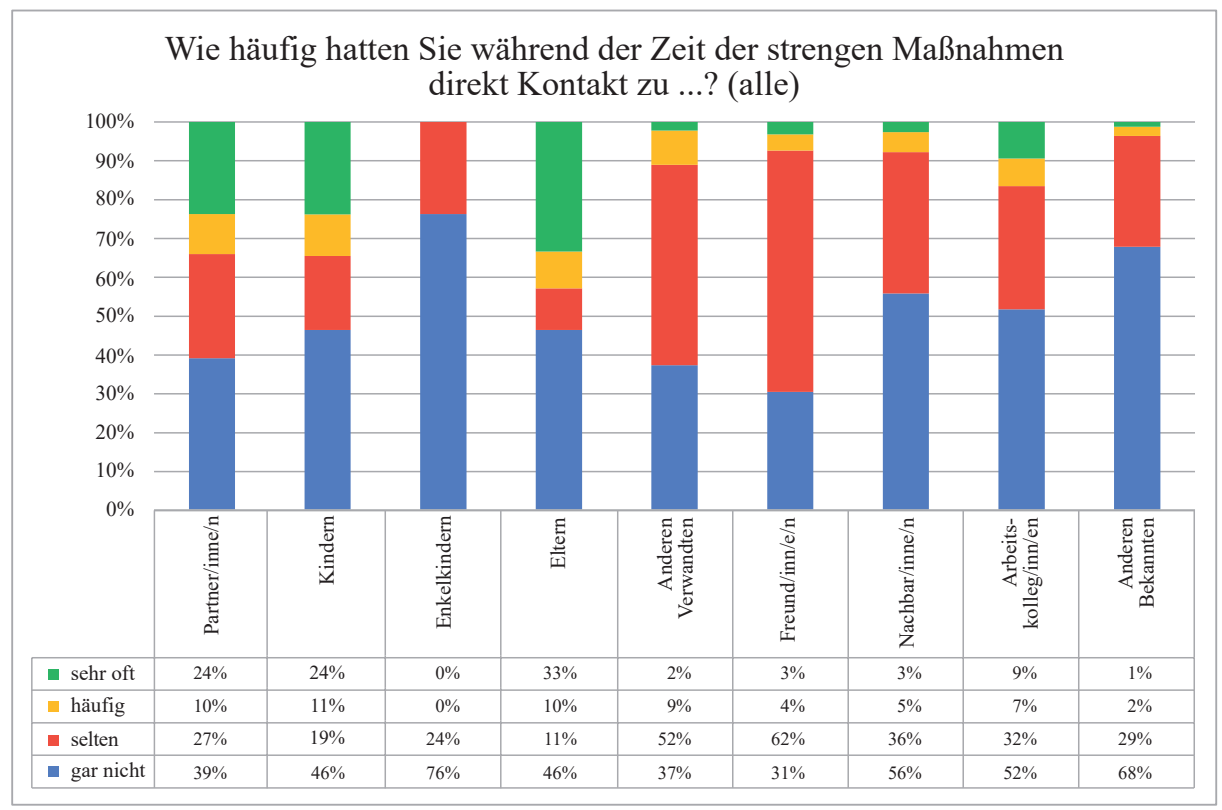

Quelle: eigene Erhebung 2020.

Die Frage nach der Häufigkeit von direkten bzw. physischen Kontakten zu anderen Personen bezieht sich insbesondere auf die Phase der strengen Maßnahmen, wie sie im Lockdown von Seiten der Regierung Mitte März 2020 verordnet wurden. Diese Maßnahmen beinhalteten im Wesentlichen die soziale bzw. physische Distanzierung zu anderen Personen außerhalb des eigenen Haushalts sowohl im öffentlichen als auch im privaten Raum.

Wie aus Abbildung 3.1 hervorgeht, hatte in jener Phase der strengen Maßnahmen eine überwiegende Mehrheit des Samples gar keinen bzw. nur seltenen Kontakt zu anderen Personen (bei allen befragten Personengruppen mehr als 56\%). Der physische Kontakt zu den beiden Gruppen „Enkelkindern“ (die Befragten bewerteten diesen zu $100 \%$ als gar nicht bzw. nur selten vorhanden) und ,anderen Bekannten“ (die Befragten bewerteten diesen zu 97\% als gar nicht bzw. nur selten vorhanden) wurde nach Angaben unserer Respondent/inn/en am seltensten praktiziert. 
Am häufigsten gaben die Respondent/inn/en direkten Kontakt zu Eltern (43\%), Kindern (35\%) und Partner/inne/n (34\%) an. Da sich die von Seiten der Regierung vorgeschriebene Verordnung der physischen Distanzierung auf Personen außerhalb des eigenen Haushalts bezog, spielt die Zusammensetzung des Haushalts der Befragten hierbei eine entscheidende Rolle. Es zeigt sich, dass häufiger direkter Kontakt zu jenen Personengruppen gepflegt wurde, die auch Teil der Haushaltsstruktur der Befragten waren (vgl. Abb.1.10.1).

Abb. 3.1.1: Häufigkeit der Sozialkontakte im Lockdown nach Personenkreisen (Herkunft Afghanistan)

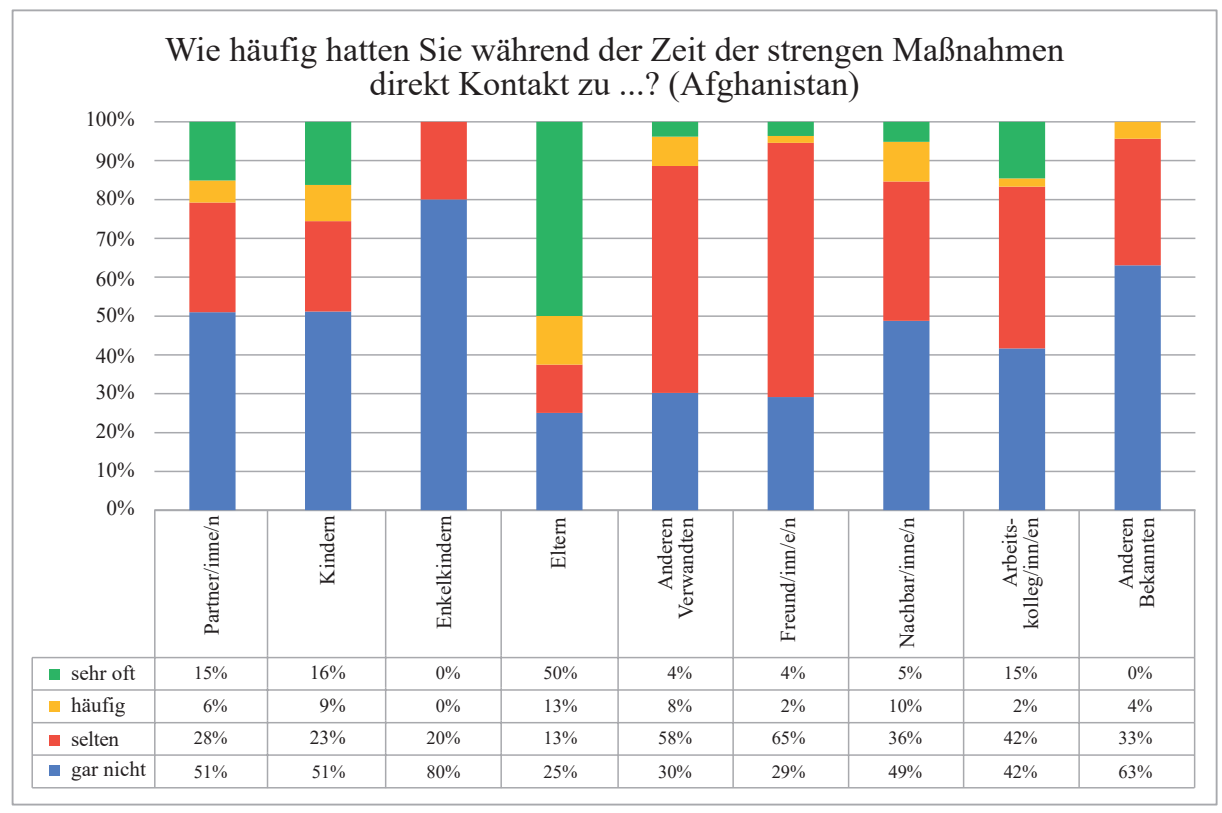

Quelle: eigene Erhebung 2020.

Innerhalb der afghanischen Community wurde der direkte Kontakt zu anderen Personen im Zeitraum der strengen Maßnahmen mehrheitlich als gar nicht bzw. nur selten angegeben. Am seltensten bzw. gar nicht wurde unter den befragten Afghan/inn/ en direkter Kontakt mit den Enkelkindern praktiziert (100\%). Diesem folgten direkte Kontakte zu anderen Bekannten (96\%), Freund/inn/en (94\%), anderen Verwandten (88\%), Nachbar/inne/n (85\%), Arbeitskolleg/inn/en, Partner/inne/n (79\%) und Kindern (74\%). Wie in Abb. 3.1.1 (Afghanistan) ersichtlich, wurde der direkte Kontakt mit den Eltern in der afghanischen Community am häufigsten praktiziert. Vergleicht man diesen Wert mit dem syrischen Teilsample, so manifestiert sich ein deutlicher Unterschied zwischen den beiden Herkunftsgruppen. Während der physische Kontakt mit den 
Eltern von den afghanischen Befragten mit einem Wert zu 63\% als sehr oft bzw. häufig klassifiziert wurde, lag dieser bei den syrischen Befragten lediglich bei 17\%. Diese Diskrepanz kann auf die Altersstruktur der Befragten sowie auf die damit zusammenhängende Haushaltsstruktur zurückgeführt werden. Der Anteil befragter Afghan/inn/en in der Gruppe der 19- bis 29-Jährigen (64\%) war besonders hoch. Dieselbe Altersgruppe war in der syrischen Subgruppe mit 20\% deutlich schwächer repräsentiert (vgl. Abb. 1.1). Zudem gaben 31\% der befragten Afghan/inn/en an, gemeinsam mit ihren Eltern zu wohnen, während von den Syrer/inne/n nur 9\% den Haushalt mit den Eltern teilten (vgl. Abb. 1.10.1).

Wie aus den qualitativen Interviews hervorging, haben Respondent/inn/en sehr individuell auf die gesetzten Maßnahmen zur Einhaltung der (physischen) Sozialkontakte reagiert. Einige Befragte artikulierten die Wichtigkeit der physischen Distanzierung aufgrund von erhöhter Ansteckungsgefahr bei persönlichem Kontakt. Die Angst vor einer Verbreitung des Virus bezog sich dabei einerseits auf die Angst, sich persönlich mit dem Virus anzustecken als auch jene das Virus zu übertragen.

Informant/inn/en und Expert/inn/en nahmen Bezug auf die Einhaltung der Maßnahmen im Familien- und Freundeskreis, wobei die diesbezüglichen Argumentationen zwischen den Familien, Bekannten oder Verwandten näher dargestellt wurden. Unter normalen Umständen wäre die Verweigerung Besuche abzustatten oder zu empfangen als sehr unhöflich aufgefasst worden. Es ging den Befragten dann darum zu zeigen, dass das Social Distancing in diesen Zeiten notwendig ist.

Vor allem der direkte Kontakt zwischen Älteren und Jüngeren wurde als risikoreiche Infektionsgefahr für ältere Personen dargestellt, da bei jungen Menschen die Infektion häufiger symptomlos und weniger schwerwiegend verläuft als bei Personen höheren Alters bzw. mit Vorerkrankung. Insbesondere jene Großeltern, die Betreuungsfunktionen gegenüber ihren Enkelkindern erfüllten (z.B. bei berufstätigen Eltern), waren genötigt den physischen Kontakt einzustellen oder sich auf Sichtkontakt auf Distanz zu beschränken.

Inwieweit eine physische Distanzierung zu Personen außerhalb des eigenen Haushalts möglich war, hing vor allem von der Umgebung ab. Dies betraf insbesondere Personen, die in großer Zahl auf kleinem Raum bzw. in geteilten Räumlichkeiten lebten, sowie auch Personen, die alleine wohnten. Beide Gruppen waren mit Schwierigkeiten konfrontiert, die gesetzten Distanzierungsmaßnahmen einzuhalten. Vor allem in den Asylunterkünften, wo Geflüchtete auf knappem Raum zusammenleben, sich Dusche und Badezimmer teilen und das Zusammenleben oftmals zu eng werden kann, hatten Betroffene Schwierigkeiten die Maßnahmen zu befolgen, wie Expert/inn/en sowohl aus der Community als auch aus dem psychosozialen Bereich erläuterten. Die Durchführung von Kontrollen seitens der Betreuer/innen, die Einhaltung körperlicher Distanz bei der Begrüßung, die Notwendigkeit sich nur in den eigenen Zimmern aufzuhalten und einander nicht zu besuchen, war für manche konfliktträchtig. Ein Experte verwies auch auf die Schwierigkeiten, wenn Familienmitglieder aufgrund der zeitverzögerten Ankunft in Österreich noch nicht 
zusammenleben konnten und einander während des Lockdowns nicht sehen konnten, so beispielsweise ein Vater, der sein neugeborenes Kind nicht besuchen konnte.

Neben den Personen, die in einer Asylunterkunft lebten, waren die strikten Distanzierungsvorgaben vor allem für jene Geflüchteten eine große Herausforderung, die alleine wohnten, ledig oder Alleinerzieherinnen waren. In den qualitativen Interviews wurde eindringlich über die Einsamkeit und Isolation der Betroffenen gesprochen. Bereits vor der Pandemie war Einsamkeit ein wesentliches Problem für viele, das sich in den Wochen des ersten Lockdowns sehr verschärfte.

Während des Lockdowns und der strengen Ausgangsbeschränkungen spielten vor allem jene Beschäftigungen eine Rolle, die dem persönlichen Zeitvertreib dienen sollten. In diesem Zusammenhang erwähnte ein/e Expert/e/in aus der Community vor allem die kulturellen Aspekte in der afghanischen Gesellschaft, in der das alltägliche Zusammenleben stark von sozialen Strukturen wie dem (erweiterten) Familien-, Freundes- und Bekanntenkreis geprägt ist und ein gemeinschaftliches Miteinander dem individualistischen Lebensstil vorgezogen wird. Expert/inn/en berichteten, dass jedoch viele ihrer Klient/inn/en aus Angst vor Ansteckung die vorgeschriebenen Distanzierungsmaßnahmen einhielten. Manche versuchten einander zwecks Psychohygiene zumindest im Freien zu treffen und die Kontakte auf einige wenige Menschen bzw. Familien zu beschränken. Mit dem Physical Distancing einher ging auch der Verlust der Deutschkenntnisse bzw. des Erlernten. Falls Jugendliche Kontakt untereinander hielten, dann großteils nur in der eigenen Herkunftsgruppe und nicht mit anderen, mit welchen sie Deutsch sprechen konnten. Daher waren auch die Online-Angebote so wichtig und wurden auch besonders nachgefragt.

Kulturelle und religiöse Orte bzw. Ereignisse, die zum sozialen Austausch und gemeinschaftlichen Miteinander einladen, waren besonders stark von den Distanzierungsmaßnahmen betroffen. Vor allem jene wichtigen kulturellen und religiösen Feste der afghanischen und muslimisch-syrischen Geflüchteten wie das Eid al-Fitr (Fest nach dem Fastenmonat Ramadan) und das Kurban Bayram (Islamisches Opferfest), welche u.a. in die Zeit der strengen Maßnahmen fielen und ansonsten gerne im erweiterten Familien-, Freundes- und Bekanntenkreis gefeiert werden, wurden verstärkt im engsten Familienbzw. Bekanntenkreis zelebriert. Neben den kulturellen bzw. religiösen Feiertagen sind es gerade auch Lebensübergangsriten, zu denen viele Besucher/inn/en geladen werden. Während der strengen Lockdown-Phase war es nicht erlaubt Hochzeiten zu feiern (nach der Lockdown-Phase waren diese zwar wieder erlaubt, jedoch mit limitierter Besucher/ inn/en/zahl) und die Teilnahme an Begräbnissen war nur dem engsten Familienkreis vorbehalten.

In den Moscheen, die anfänglich in der „heißen Phase“ des Lockdown gänzlich geschlossen waren, wurden anschließend strenge Regeln erlassen. Die Räumlichkeiten wurden desinfiziert, Besuchenden wurde aufgetragen ihren eigenen Gebetsteppich mitzubringen und Frauen wurden in manchen Moscheen nicht zugelassen. Jenes Betreuungsprogramm, welches in einer Wiener Moschee an den Wochenenden für Frauen und 
Kinder veranstaltet wurde und die Möglichkeit für gegenseitigen Austausch sowie für gemeinsames Essen und Farsi-Lernen etc. bot, wurde gestrichen. Vor allem für jene Frauen, die noch nicht lange in Österreich waren, keine Deutsch- und/oder Englischkenntnisse und wenig Sozialkontakte besaßen, boten diese Treffen eine wichtige Unterstützung, um sich in der neuen Umgebung zurechtzufinden, wie ein Vertreter der Islamischen Glaubensgemeinschaft erläuterte.

Die Einhaltung der Distanzierungsvorschriften und die Reduzierung der Sozialkontakte auf jene Personen, mit denen der eigene Haushalt geteilt wird, hatte jedoch auch Auswirkungen auf das gemeinsame Zusammenleben und barg u.a. Konfliktfelder in sich. Respondent/inn/en schilderten Partnerschaftsprobleme, die aus der intensiven, gemeinsam verbrachten Zeit resultierten, sogar Scheidungen wurden während der Lockdown-Phase eingereicht.

Abb. 3.1.2: Häufigkeit der Sozialkontakte im Lockdown nach Personenkreisen (Herkunft Syrien)

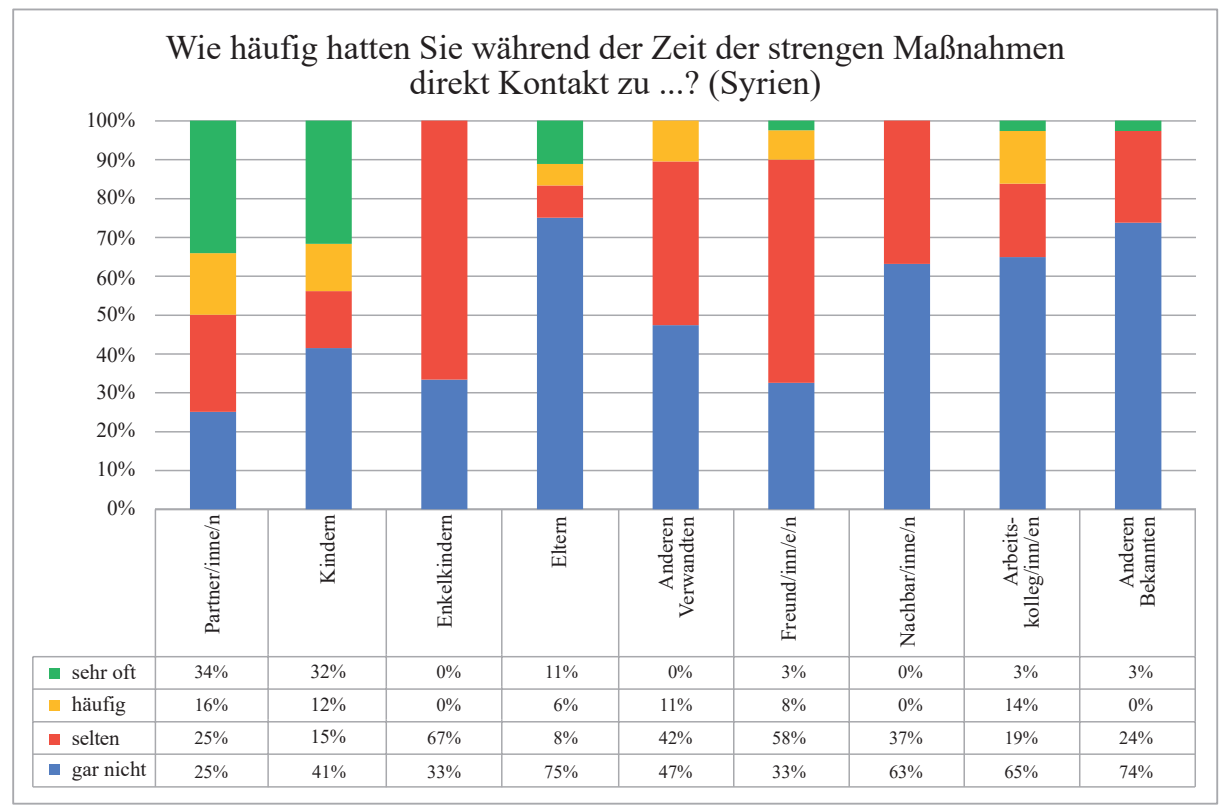

Quelle: eigene Erhebung 2020.

Innerhalb der syrischen Community wurde der direkte Kontakt mit Enkelkindern und Nachbar/inne/n am seltensten praktiziert (vgl. Abb. 3.1.2). Dem folgen seltene Kontakte mit anderen Bekannten (98\%), Freund/inn/en (91\%), anderen Verwandten $(89 \%)$, Arbeitskolleg/inn/en (84\%) und Eltern (83\%). Dies erklärt sich durch das Faktum, dass sich im Sample wenige Dreigenerationenfamilien befinden bzw. dass 
nur selten drei Generationen nach Österreich geflüchtet sind. Des Weiteren ging aus den Analysen hervor, wie sehr die Befragten von den Sozialkontakten in ihren jeweiligen Netzwerken (syrischen und österreichische Freund/inn/e/n, Bekannte) abhängig waren und wie eingeschränkt dies während des ersten Lockdowns nur möglich war. Am häufigsten hatten Syrer/innen direkten Kontakt mit Partner/inne/n (50\%) und Kindern (44\%) (vgl. 3.1.2 Syrien). Vergleicht man diese Werte mit der Haushaltsstruktur des syrischen Teilsamples, so zeigt sich, dass auch der Anteil der gemeinsam geteilten Haushalte mit Partner/inne/n und Kindern (28\%) hier am größten ist (vgl. Abb. 1.10.1).

Abb. 3.2: Häufigkeit der virtuellen Kontakte im Lockdown nach Personenkreisen (gesamt)

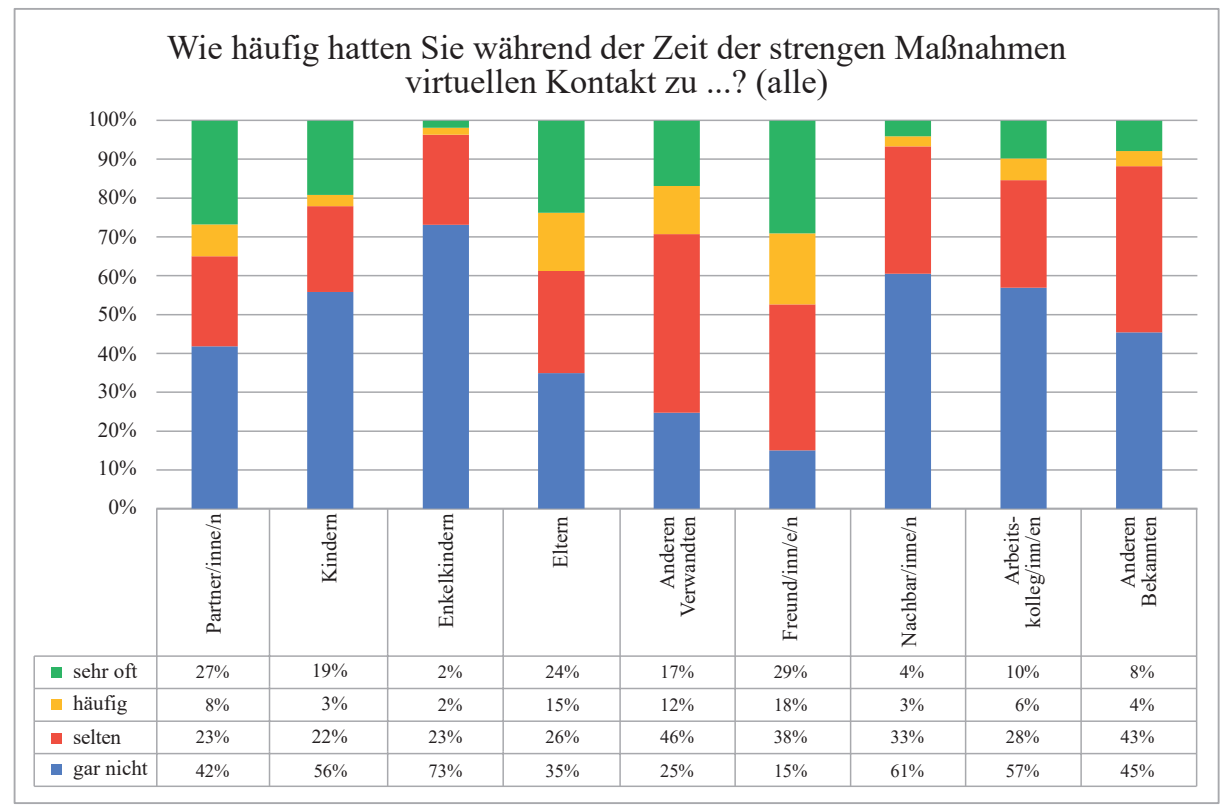

Quelle: eigene Erhebung 2020.

Da physische Kontakte während des Lockdowns stark eingeschränkt waren, wurden im Rahmen der Umfrage auch die virtuellen Kontakte der Respondent/inn/en abgefragt.

Wie aus Abbildung 3.2 hervorgeht, dominierten virtuelle Kontakte zu Freund/inn/ en mit einer Häufigkeit von 47\% vor jenen zu den Eltern, der mit 39\% sehr oft bzw. häufig praktiziert wurde. Darauf folgen virtuelle Kontakte zu den Partner/inne/n (35\%), anderen Verwandten (29\%), Kindern (22\%), Arbeitskolleg/inn/en (16\%) und anderen Bekannten (12\%). Am seltensten bzw. gar nicht wurden virtuelle Kontakte zu Nachbar/ inne/n (7\%) und Enkelkindern (4\%) gepflegt. 
Betrachtet man die Häufigkeit der virtuellen Kontakte zu den angegebenen Personengruppen im Teilsample Afghanistan, so zeigen sich keine wesentlichen Unterschiede zu jenen im o.a. Gesamtsample (vgl. Abb. 3.2.1). Auch innerhalb der afghanischen Community dominierte der virtuelle Kontakt zu Freund/inn/en (52\%) vor den virtuellen Kontakten zu den Eltern (36\%), anderen Verwandten (32\%), Partner/inne/n (31\%), Arbeitskolleg/inn/en (18\%), Kindern (15\%) und anderen Bekannten (10\%). Wie auch im Gesamtsample, so wurden auch im Teilsample Afghanistan Kontakte auf virtueller Basis zu Nachbar/inn/en (4\%) am seltensten bzw. zu Enkelkindern gar nicht (0\%) praktiziert, da aufgrund der jungen Altersstruktur nicht vorhanden.

In den qualitativen Interviews zeigte sich, dass Online-Kommunikationsportale in allen Altersgruppen weit verbreitet sind. Sie dienten insbesondere in den Zeiten der Pandemie als soziale Vernetzungs- und Austauschforen sowie als Informationsplattformen. Sogenannte Messenger-Dienste, wie WhatsApp, Viber, Telegram etc., ermöglichen somit eine rasche Kontaktaufnahme zu Familie und Verwandtschaft im Ausland. Um das Gegenüber im Gespräch auch sehen zu können, wurden vor allem jene oben genannten Dienste mit Videofunktion bevorzugt.

Abb. 3.2.1: Häufigkeit der virtuellen Kontakte im Lockdown nach Personenkreisen (Herkunft Afghanistan)

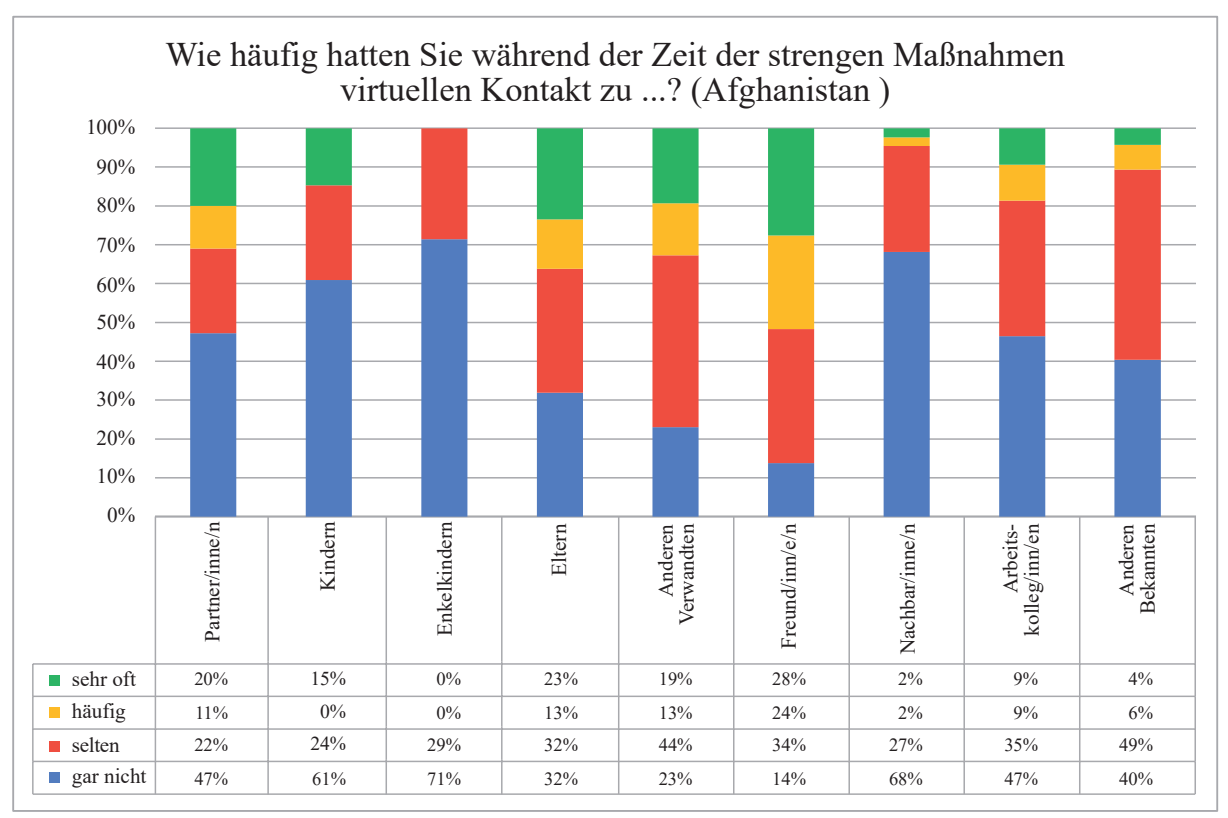

Quelle: eigene Erhebung 2020. 
Diese intensivierten Kontakte über soziale Medien sind jedoch nicht erst seit COVID-19 zu beobachten, sondern schon davor. Familienmitglieder sind über mehrere Länder verstreut und schon alleine deshalb werden diese Dienste verstärkt genutzt. Geändert hat sich allerdings das Nutzungsverhalten, da auch ältere Personen nun vermehrt auf diese Dienste zugreifen bzw. sich die notwendigen Kompetenzen angeeignet haben.

Die Wichtigkeit der virtuellen Kontaktaufnahme zu Freunden und Freundinnen zeigte sich vor allem bei jüngeren Respondent/inn/en (auch in der Online-Erhebung wurden Freund/inn/e/n als wichtigste virtuelle Kontaktgruppe angeführt, was vor allem auf die junge Altersstruktur der befragten Afghan/inn/en rückschließen lässt), da alltägliche Sozialkontakte außerhalb der (familiären) Wohnsituation stark eingeschränkt waren.

Vor allem jene Freund/inn/e/n, die den Befragten ein gutes Gefühl in Zeiten der Unsicherheit vermitteln konnten, wurden kontaktiert. So erzählte eine junge Respondentin von den täglichen Telefonaten mit ihrer ehemaligen Betreuerin in Innsbruck, die ihr beim Fußfassen in Österreich geholfen und sie seither in jeglicher Problemsituation unterstützt hatte und dies nun auch in der Pandemiekrise fortsetzte.

Abb. 3.2.2: Häufigkeit der virtuellen Kontakte im Lockdown nach Personenkreisen (Herkunft Syrien)

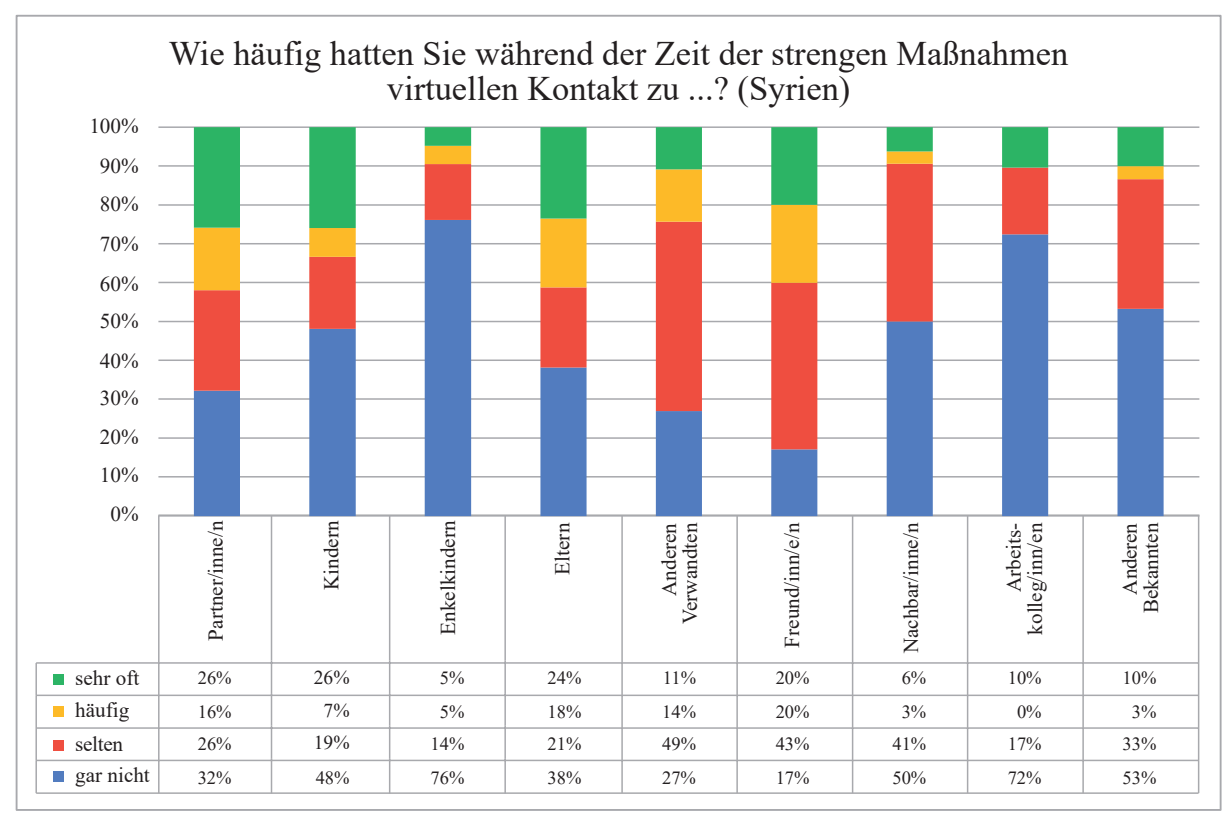

Quelle: eigene Erhebung 2020. 
In der syrischen Herkunftsgruppe weichen die Häufigkeiten der virtuellen Kontakte zu den jeweils abgefragten Personengruppen nur minimal von jenen im Gesamtsample bzw. im Teilsample Afghanistan ab (vgl. Abb. 3.2.2). Am häufigsten gaben Syrer/inn/ en virtuellen Kontakt zu den beiden Gruppen „Partner/inn/en“ und „Eltern“ an, welcher mit jeweils $42 \%$ bewertet wurde. Darauf folgen virtuelle Kontakte zu Freund/inn/en, Kindern und anderen Verwandten mit einer Häufigkeit von $40 \%, 33 \%$ und $25 \%$. Am seltensten gaben syrische Befragte virtuellen Kontakt zu anderen Bekannten (13\%), Arbeitskolleg/inn/en (10\%) und Nachbar/inne/n (9\%) an.

\subsection{Sozialkontakte und Haushaltsformen}

In der Online-Erhebung wurde auch die Frage gestellt: „Wenn Sie daran denken, wie sich Ihre sozialen Kontakte in der Corona-Krise verändert haben - was traf da auf Sie zu?" Vor allem die Verteilungen der Antworten auf die Items ,,ich vermisste Umarmungen“" sowie ,ich vermisste Besuche“ dokumentieren, dass die Haushaltsstruktur einen wichtigen Einfluss auf das subjektive Empfinden der durch Corona veränderten sozialen Interaktionsmöglichkeiten hat. In beiden Fällen waren es die in Einpersonenhaushalten lebenden Geflüchteten, die sich am stärksten betroffen fühlten. 47,4\% von ihnen vermissten Umarmungen sehr stark, fast 58\% vermissten am stärksten die Besuche. Dabei war auch das Zusammenleben mit den Eltern kein Faktor, der diese Defizite nicht dennoch spürbar gemacht hätte, wenngleich in geringerem Maße. Auch $48 \%$ der Befragten, die im elterlichen Haushalt wohnten, sowie 42\% jener mit einer eigenen Familie vermissten Umarmungen. Bezüglich des Vermissens von Besuchen lagen die Anteile bei im elterlichen Haushalt lebenden Befragten bei fast 45\%, bei den mit der eigenen Familie wohnenden Interviewten bei 59\%. Die Werte für in Wohnheimen sowie in WGs lebenden Geflüchteten waren hier deutlich niedriger.

Hinsichtlich des Bedarfs an Kontakten mit Menschen, bei denen man sich wohlfühlt, zeigten die Alleinlebenden ein hohes Antwortrating in der Kategorie „trifft stark zu“" $(52,3 \%)$, wobei dies von jenen übertroffen wurde, die mit den Eltern zusammenlebten (76,7\%). Auch die in Familienhaushalten wohnenden Interviewees haben zu 73\% die Kategorie „trifft stark zu“ angekreuzt. Seltener wurde das Bedürfnis nach Menschen, bei denen man sich wohlfühlt, von Befragten in Wohngemeinschaften und am wenigsten von den in Wohnheimen Lebenden angemerkt.

Eine weitere Facette der Folgen der Veränderungen sozialer Interaktionen infolge des Lockdowns wurde durch das Item ,ich vermisste es allgemein, unter Menschen zu sein" erhoben. Der Spitzenwert $(57,1 \%)$ fand sich bei den in Wohngemeinschaften wohnhaften Befragten, 53\% der im Haushalt der Eltern sowie 54,3\% der in Familienhaushalten lebenden Geflüchteten haben diese Kategorie ebenfalls als stark zutreffend markiert. Das Antwortrating in der Kategorie Singlehaushalte war niedriger. Der vermeintliche Widerspruch bei Menschen, die in Wohngemeinschaften lebten, und 
ihrem Bedürfnis ,allgemein unter Menschen zu sein“ bezog sich auf die sozialen Kontakte außerhalb des gemeinsamen Haushaltes.

Hinsichtlich der Verfügbarkeit von Hilfestellungen, erfasst im Item „es gab genug Menschen, die mir geholfen hätten, wenn ich Probleme hatte“, zeigt sich ein anderes Bild. Die höchsten Anteile in der Kategorie „trifft gar nicht zu“ entfallen hier auf Befragte, die in WGs oder Wohnheimen leben (30,8\% bzw. 25\%). Diese verfügen offenbar noch nicht in einem hohen Ausmaß über soziale Netzwerke, auf die sie im Notfall zurückgreifen könnten. In der Befragung wurde auch nicht näher spezifiziert, ob es sich dabei um selbst organisierte oder von NGOs betriebenen WGs handelt und auch nicht, welche Betreuungsstruktur in den einzelnen Wohnheimen organisiert war. Auffällig ist daher das Gefühl des „Alleingelassenseins“ und der gefühlt geringeren Einbindung in soziale Netzwerke. Tragfähiger sind die Netzwerke bei in Einpersonenhaushalten Lebenden, von denen 52,4\% die Kategorie ,trifft stark zu“" gewählt haben. Dieser Wert lag über jenem der mit der eigenen Familie (41\%) oder noch bei den Eltern $(34,5 \%)$ lebenden Befragten.

Dass man „sich häufig im Stich gelassen fühlte“, traf auf die Alleinlebenden sowie die Befragten in Familienhaushalten am stärksten zu. Erstere haben mit fast 53\% auch den höchsten Wert in der Kategorie ,trifft ein wenig zu“. In WGs lebende Personen sind hier mit $40 \%$ präsent. $48,2 \%$ der bei den Eltern wohnhaften und 52,2\% der mit eigener Familie zusammenwohnenden Befragten haben dies als gar nicht zutreffend klassifiziert.

Menschliche Geborgenheit und Wärme wurde von den Alleinlebenden am ehesten vermisst. Fast 29\% wählten hier die Kategorie ,trifft stark zu“ und auf 33,3\% traf dies teilweise zu. Auf zwei Drittel der in Wohnheimen lebenden Befragten traf dies ein wenig zu, am wenigsten Probleme hatten hier die im Familienverband wohnhaften Personen, auf die dies zu 42,3\% gar nicht zutraf.

Viele Menschen, auf die man sich wirklich verlassen kann, kannten in der Familie (51,7\%) lebende Respondent/inn/en, etwas weniger (50\%) in Wohnheimen Lebende, Singles (47,6\%) und am wenigsten jene Befragten (39,3\%), die noch im Haushalt der Eltern lebten. Die Kategorie ,trifft gar nicht zu“ haben andererseits am häufigsten (25\%) jene gewählt, die in Wohnheimen ansässig waren.

Die teils kontradiktorischen Aussagen über das Leben in gemeinsamen Haushalten und die Gefühle von zu wenigen sozialen Kontakten und Unterstützungsoptionen müssten noch genauer untersucht werden. Inwieweit da die COVID-19 Situation eine spezielle Rolle gespielt hat oder ob es diese Einschätzungen bereits vor der Pandemie gab, ist aus den vorliegenden Befragungen zwar im Groben erkennbar (siehe 3.2.), aber nicht im Detail zu eruieren. Auch sind keine vergleichbaren Daten über die Befindlichkeiten von Personen, die keine Fluchterfahrung haben, vorhanden. Die uni- und bivariaten Analysen kreisen jedoch um die Problematik von Einsamkeit und das Vermissen von tieferen, tragfähigen sozialen Kontakten. 
Dass „es genug Menschen gibt, denen ich mich eng verbunden fühle“ haben vor allem solche, die mit den Eltern zusammenleben (48,2\%) und in Einpersonenhaushalten lebende Befragte (42,9\%) als für sie stark zutreffend markiert. Allerdings war die Kategorie ,trifft gar nicht zu“ mit $14,3 \%$ bei den Personen in Singlehaushalten am stärksten besetzt.

Keinen eindeutigen Trend zeigte die Antwortverteilung beim Item „mir fehlten Menschen, mit denen ich mich über die Coronasituation austauschen kann“. Stark traf dies auf 25\% der Alleinlebenden zu, allerdings erreichten diese mit einem Drittel und die in Wohnheimen Lebenden mit 50\% die höchsten Werte in der Kategorie „trifft gar nicht zu“. Ein wenig traf dies auf 50\% der in Asylquartieren lebenden Befragten, auf ein Drittel der Alleinlebenden sowie auf 29,6\% der bei den Eltern wohnhaften Respondent/ inn/en zu.

Das Fehlen von Menschen, mit denen eine Verbesserung der Deutschkenntnisse möglich gewesen wäre, traf auf alle Wohnformen mit sehr hohen Werten zu. In erster Linie auf Personen in Wohngemeinschaften $(38,5 \%)$, aber auch auf jene in Familienverbänden (34,5\%), auf jene in Einpersonenhaushalten $(31,6 \%)$ und in elterlichen Haushalten (über 30\%). Hingegen viel weniger auf jene in Wohnheimen (20\%), in denen ja der Kontakt mit österreichischem Personal auch während der Coronakrise eher möglich war.

Das Fehlen von Sexualpartner/inne/n stellte ein geringes Problem dar. Es wurde selten als stark zutreffend klassifiziert, teilweise zutreffend von Bewohner/inne/n von Wohnheimen, aber auch seitens der Befragten in Familienhaushalten und bei den Eltern Lebenden.

\subsection{Veränderung von Sozialkontakten in der Coronakrise}

Um die Auswirkungen der Einschränkung der sozialen bzw. physischen Kontakte zu untersuchen, haben wir die Respondent/inn/en zu den Veränderungen in ihrem Sozialleben seit Beginn der Corona-Pandemie befragt. Wie Abb. 3.3. veranschaulicht, hat eine Mehrheit der Befragten folgende Aussagen als völlig bzw. stark zutreffend bewertet: $62 \%$ der Befragten vermissten Leute, bei denen sie sich wohlfühlen. $52 \%$ vermissten es allgemein unter Menschen zu sein. 47\% gaben an Besuche zu vermissen und $41 \%$ der Befragten vermissten Umarmungen. Andererseits gaben 45\% der Befragten an, viele Menschen zu kennen, auf die sie sich wirklich verlassen konnten. Während $41 \%$ meinten, dass es genügend Menschen gäbe, mit denen sie sich eng verbunden fühlten, gaben $40 \%$ des Gesamtsamples an, dass es genügend Menschen gäbe, die ihnen geholfen hätten, wenn sie Probleme hatten. Das Fehlen von Menschen, mit denen die eigenen Deutschkenntnisse weiter gepflegt werden konnten, haben 33\% der Befragten als völlig bzw. stark zutreffend formuliert, 53\% gaben jedoch an, dass dies für sie gar nicht oder nur ein wenig zutreffe und 14\% klassifizierten dies für sich als teilweise zutreffend. 
Abb.: 3.3: Kategorien der Veränderung von Sozialkontakten in der Coronakrise (gesamt)

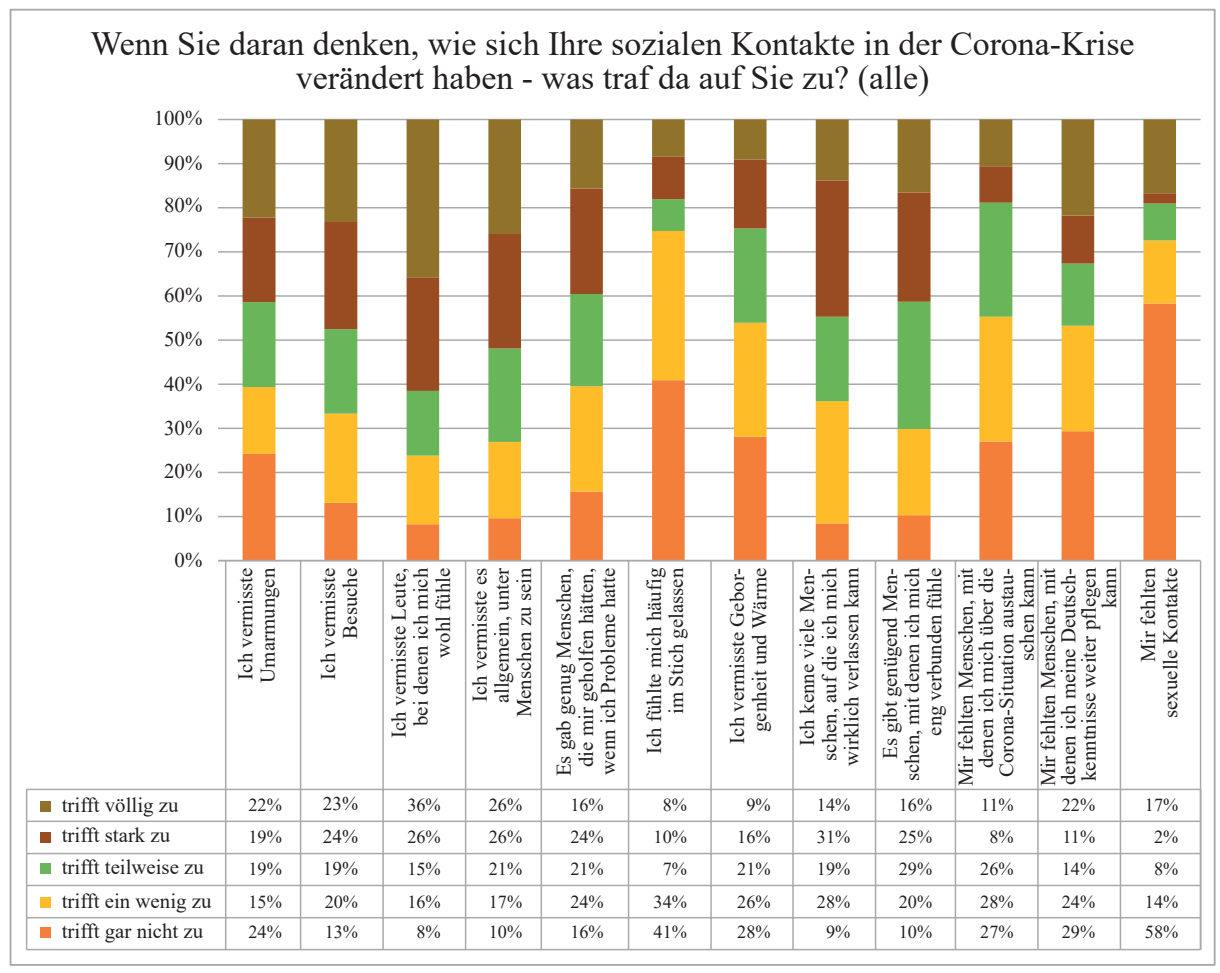

Quelle: eigene Erhebung 2020.

Als weniger bzw. gar nicht zutreffend wurden das Vermissen von Geborgenheit und Wärme (54\%), das Fehlen von Menschen, mit denen man sich über die Corona Situation austauschen konnte (55\%), das Fehlen von sexuellen Kontakten (72\%), sowie das Sich-häufig-im-Stichgelassen-Fühlen (75\%) bewertet.

Von den afghanischen Befragten (vgl. Abb. 3.3.1) gab eine große Mehrheit an, dass sie Leute vermissten, bei denen sie sich wohlfühlten (62\%), allerdings hatten $50 \%$ das Gefühl, dass es genügend Menschen gab, mit denen sie sich verbunden fühlten. Weitere 49\% vermissten es unter Menschen zu sein (49\%) sowie Besuche im Allgemeinen (40\%).

Am wenigsten hatten afghanische Befragten das Gefühl im Stich gelassen zu werden (89\% gaben dies als wenig bzw. gar nicht zutreffend an) oder vermissten sexuelle Kontakte (78\% wenig/gar nicht zutreffend). Zudem wurde das Vermissen von Geborgenheit und Wärme von $62 \%$, das Fehlen von Menschen, mit denen man sich über Corona austauschen konnte von $61 \%$ und das Fehlen von Menschen, mit denen man seine Deutschkenntnisse weiter pflegen konnte, von $60 \%$ als wenig bzw. gar nicht 
Abb. 3.3.1: Kategorien der Veränderung von Sozialkontakten in der Coronakrise (Herkunft Afghanistan)

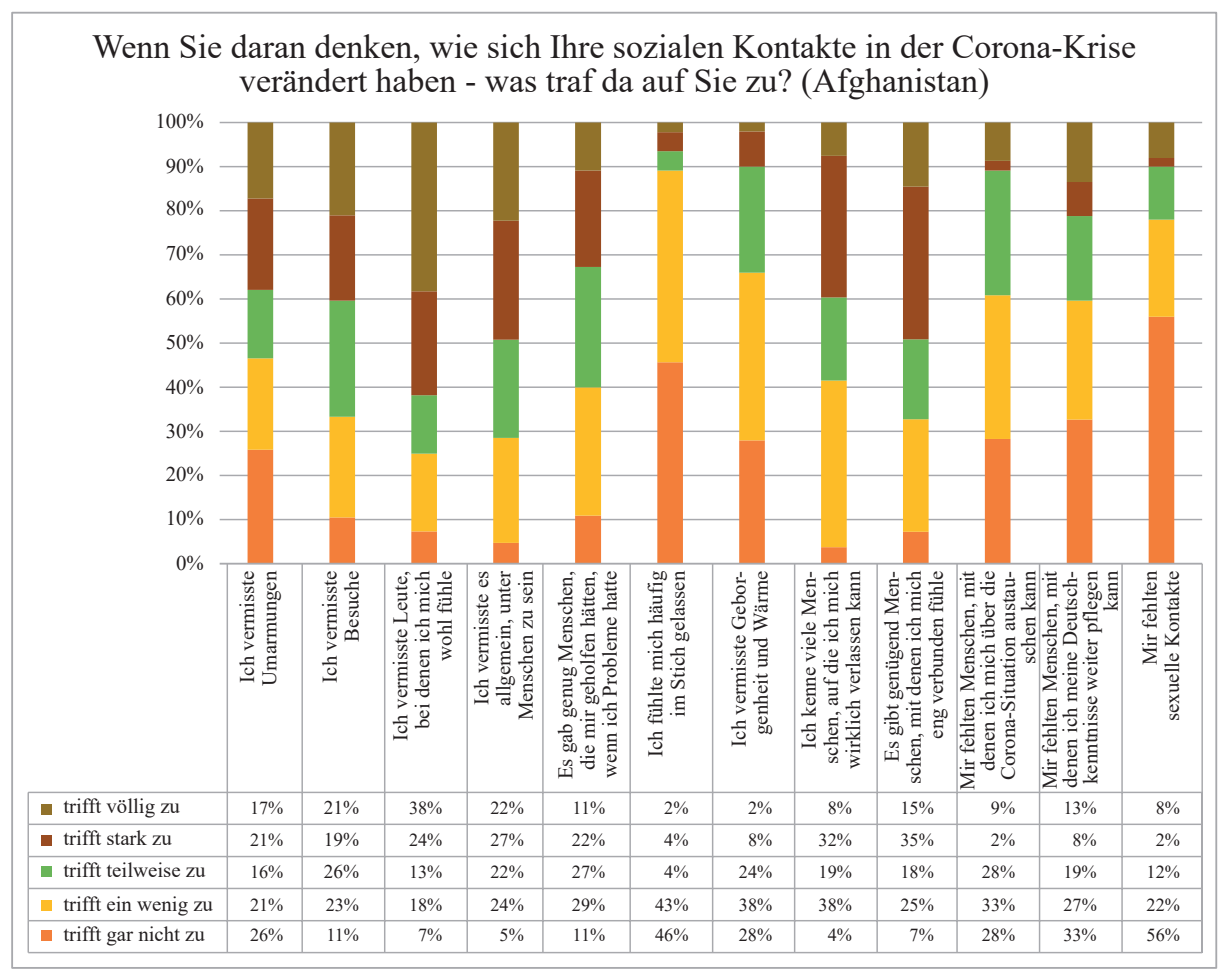

Quelle: eigene Erhebung 2020.

zutreffend beantwortet. Hinsichtlich des Vermissens von Umarmungen gaben $47 \%$ wenig/gar nicht zutreffend an, $16 \%$ als teilweise und 38\% als stark/völlig zutreffend. $42 \%$ meinten, dass es nicht bzw. wenig zuträfe, dass sie Menschen kennen auf die sie sich wirklich verlassen konnten, 19\% teilweise und 40\% trifft völlig bzw. stark zu. Dass es genug Menschen gäbe, die ihnen geholfen hätten, wenn sie Probleme hatten, meinten $40 \%$ als nicht bzw. wenig zutreffend, $27 \%$ teilweise und 33\% völlig bzw. stark zutreffend.

Die Auswirkungen der veränderten sozialen Kontakte seit Beginn der CoronaPandemie, die vor allem auf das alltägliche Gefühlsleben der Geflüchteten Einfluss nahmen, wurden auch in den qualitativen Interviews adressiert. Vor allem die physische Distanzierung gegenüber engen Freund/inn/en evozierte das Vermissen von körperlicher Nähe als Ausdruck freundschaftlicher Zuneigung und das Gefühl, dass diese Form der Beziehung nicht mehr so unbeschwert war. Soziale Isolation und Gefühle von Einsamkeit waren vor allem für alleinlebende Respondent/inn/en vorherrschend, die zudem auch negative Gefühlserinnerungen an vergangene Fluchterlebnisse hervor- 
riefen. Positiv vermerkt wurde, dass über soziale Medien Kontakte zu Menschen, von denen man schon lange nichts mehr gehört hatte, wieder aufgegriffen wurden.

Auch eine Expertin im Asylbereich berichtete von sozialer Isolation und Gefühlen von Einsamkeit bei Geflüchteten, die jedoch aufgrund der knappen Betreuungszeit am Telefon nicht weiter besprochen werden konnten. Die Zeiten der Krise offenbarten jedoch auch Qualitäten von Freundschaften und es zeigte sich, dass manche Freundschaften diesen Belastungen nicht standhielten.

Allgemein hat das soziale Leben im öffentlichen Raum stattgefunden, welcher die Legitimität schuf, sich auch mit anderen Personen außerhalb des eigenen Haushalts zu treffen. Eine Expertin erklärte, dass das alltägliche Leben von manchen Personen ohnehin schon vermehrt auf öffentlichen Plätzen stattfand, einerseits aufgrund der oftmals knappen Wohnverhältnisse (Asylunterkunft, Großfamilien in zu kleinen Wohnungen usw.) und andererseits aufgrund der unterschiedlichen Bedeutungen des öffentlichen Raumes. Gerade die Einschränkungen im öffentlichen Raum wurden dann auch als besonders einschneidend wahrgenommen.

Abb. 3.3.2: Kategorien der Veränderung von Sozialkontakten in der Coronakrise (Herkunft Syrien)

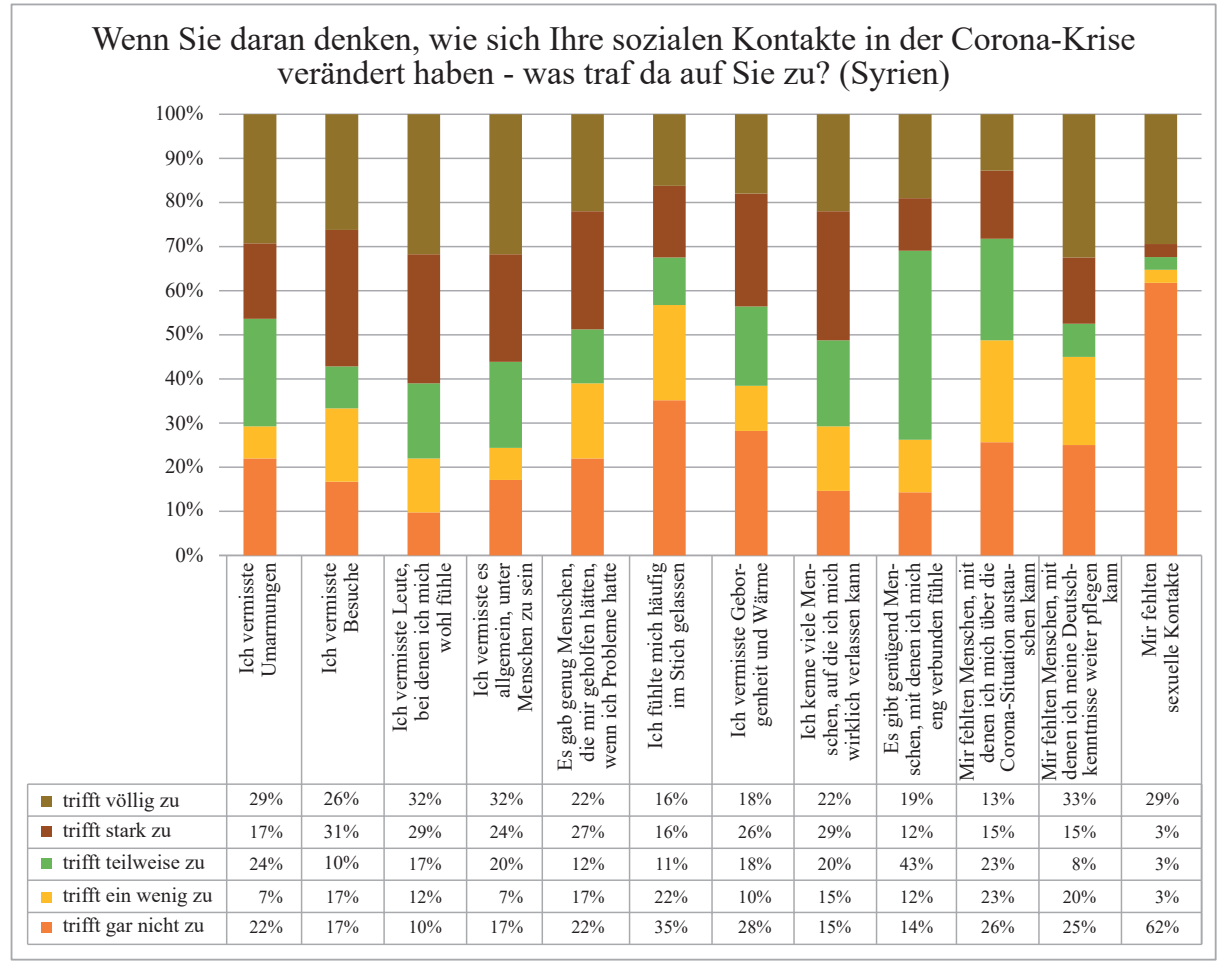

Quelle: eigene Erhebung 2020. 
Wie in der befragten Subgruppe Afghanistan vermissten Syrer/innen Leute, bei denen sie sich wohlfühlten, am meisten (61\%) (vgl. Abb. 3.3.2). Zudem vermissten $57 \%$ Besuche und allgemein unter Menschen zu sein (56\%). Auch gaben befragte Syrer/ innen vermehrt an, dass sie viele Menschen kannten, auf die sie sich wirklich verlassen konnten (51\%). Einer knappen Mehrheit (48\%) fehlten im Zeitraum der strengen Maßnahmen Menschen, mit denen sie ihre Deutschkenntnisse weiter pflegten, allerdings gaben $45 \%$ an, dass dies für sie nicht zutraf.

Eine knappe Mehrheit bestätigte, dass es genug Menschen gab, die ihnen bei Probleme geholfen hätten (49\% trifft völlig/stark zu, 12\% teilweise, 33\% trifft wenig/ gar nicht zu). Anders als bei den Afghan/inn/en gaben 64\% des syrischen Teilsamples an, Umarmungen zu vermissen, zudem vermissten $44 \%$ auch Wärme und Geborgenheit (18\% teilweise und 38\% gar nicht bzw. kaum zutreffend). Eine Mehrheit gab an, dass es für sie nur teilweise zutraf, dass es für sie genügend Menschen gab, mit denen sie sich verbunden fühlten (31\% trifft völlig/stark zu, 26\% trifft gar nicht/wenig zu). Am wenigsten fühlten sich Syrer/innen im Stich gelassen (57\%). Auch fehlten den meisten keine sexuellen Kontakte (65\%) oder Personen für den Austausch über die CoronaSituation (49\%).

\subsection{Emotionale und psychische Folgewirkungen der Corona- krise}

Um die Auswirkungen der eingeschränkten (physischen) Sozialkontakte auf ihre emotionale und psychische Befindlichkeit vertiefend zu untersuchen, wurden die Respondent/inn/en zu ihrer Gefühlslage seit Beginn der Corona-Situation befragt, welche diese im Rahmen einer Häufigkeitsskala (sehr oft-oft-manchmal-selten-nie) bewerteten. Wie Abb. 3.4 illustriert, sorgten sich die Respondent/inn/en allgemein öfter um Angehörige und Mitmenschen als um sich selbst. Am öftesten gaben die Befragten an, dass sie sich um Angehörige im Herkunftsland ängstigten (53\%), gefolgt von Sorgen um jene Angehörigen, die noch in den Flüchtlingslagern lebten (40\%). Im mittleren Bereich sind Ängste um die Familie in Österreich zu verorten, welche die Befragten mit 34\% als (sehr) oft, 26\% als manchmal und 39\% als selten bzw. nie gegeben sahen.

Die geringste Rolle spielten für die Befragten das Beten bzw. Hören von spirituellen/religiösen Podcasts (72\%). Auch die Erinnerung an die eigene Flucht wurde von einer großen Mehrheit (67\%) als selten bzw. nie angeführt. Obwohl Krisenzeiten negative Gefühle und individuelle Coping-Strategien abverlangen, fühlte sich eine Mehrheit des Gesamtsamples motiviert (66\% gaben an, dass sie sich nur sehr selten zu nichts aufraffen konnten) und negierte Gefühle von Einsamkeit (63\%), Niedergeschlagenheit (62\%) und Unsicherheit (58\%). Des Weiteren zeigten sich bei den Befragten selten Gefühle von schlechter Laune (54\%), Anstrengung im Allgemeinen (52\%), Schlafprobleme (52\%) und Angst (50\%). Eine Mehrheit gab zudem an (51\%), dass sie 
Abb. 3.4: Emotionale und psychische Folgewirkungen der Coronakrise (gesamt)

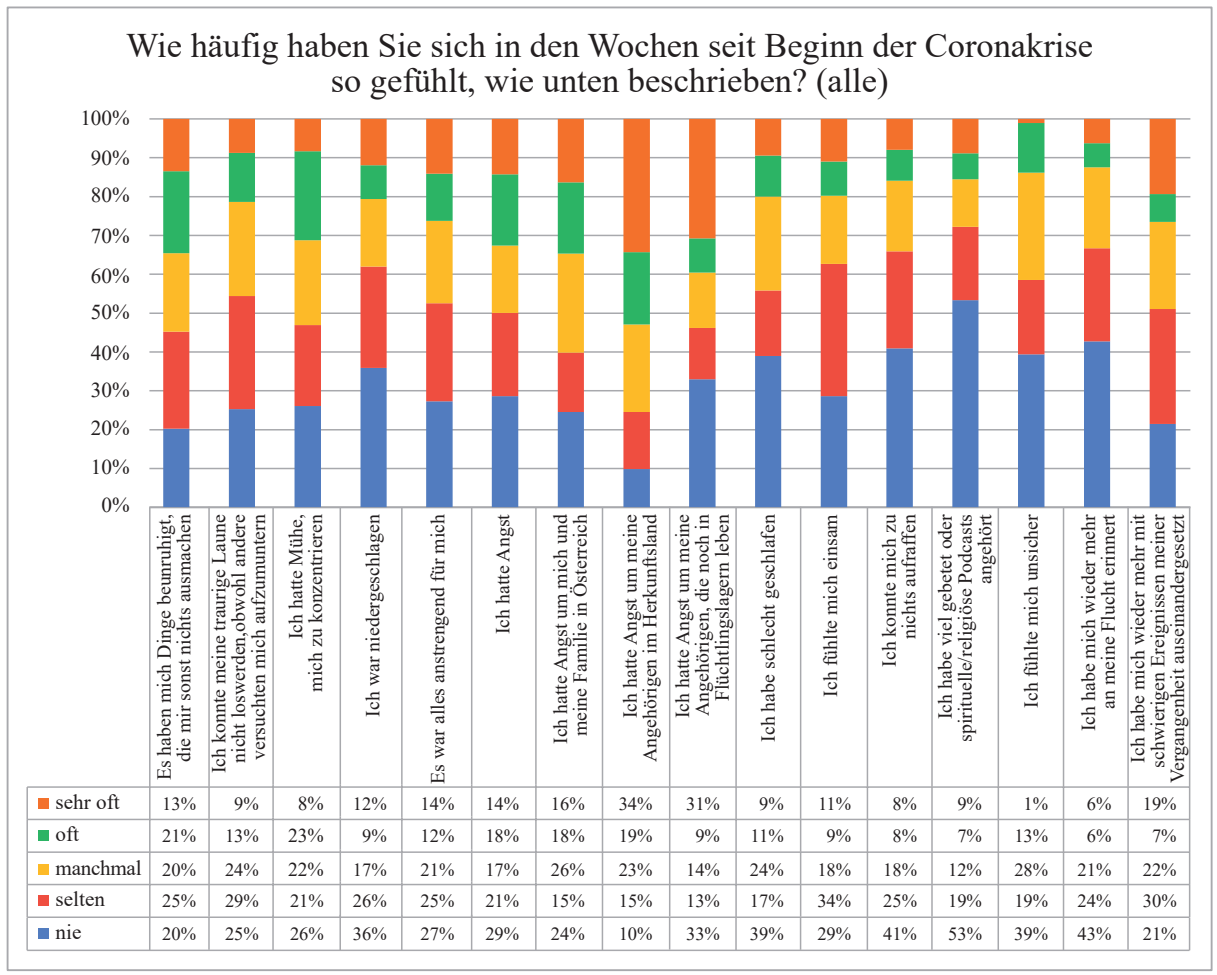

Quelle: eigene Erhebung 2020.

sich auch während der Pandemie selten oder nie mit schwierigen Ereignissen aus der Vergangenheit auseinandergesetzt hatten. Auch zeigte sich, dass sich die Respondent/ inn/en nur selten wegen Angelegenheiten beunruhigten, die ihnen auch sonst nichts ausmachten (45\%) und sie selten oder nie Mühe hatten sich im Alltag zu konzentrieren $(47 \%)$.

Eine Mehrheit der afghanischen Befragten gab am öftesten an, dass sie sich um Angehörige im Herkunftsland ängstigten (vgl. Abb. 3.4.1). Hierfür sprachen die hohen Infektions- und Mortalitätsraten der COVID-19 Erkrankten in Afghanistan und im Iran, die restriktiven Maßnahmen zur Eindämmung des Virus sowie ein überfordertes Gesundheitssystem in beiden Ländern. Angst um die Familie in Österreich empfanden $36 \%$ der Afghan/inn/en sehr oft/oft (23\% manchmal und 41\% selten oder nie). Auch die Angst um Angehörige in den Flüchtlingslagern fühlten 35\% (sehr) oft, 52\% gaben an, dass diese selten bzw. nie gegeben war. 
Abb. 3.4.1: Emotionale und psychische Folgewirkungen der Coronakrise (Herkunft Afghanistan)

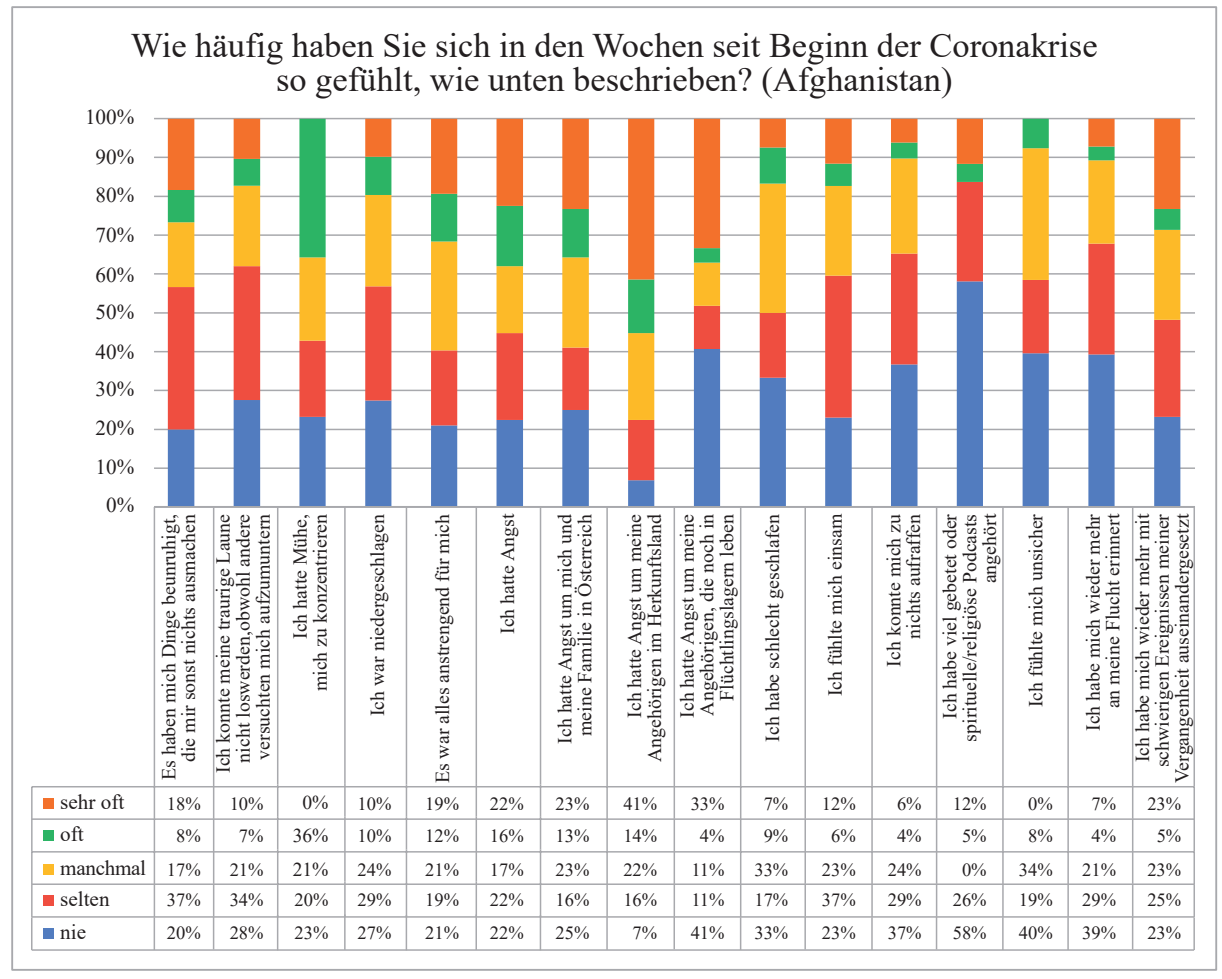

Quelle: eigene Erhebung 2020.

Am seltensten wurde von afghanischen Befragten gebetet oder spirituelle/ religiöse Podcasts gehört (84\%). Von einer Mehrheit wurde auch als selten bzw. nie vermerkt, das sie sich mit der eigenen Vergangenheit, vor allem mit den Erinnerungen an die eigene Flucht (68\% selten/nie und 21\% manchmal) auseinandergesetzt hätten. Wenige erklärten, dass die Situation während des ersten Lockdowns für sie mit fehlender Motivation (66\% selten/nie und 24\% manchmal), einer traurigen Laune (62\% selten/nie), Gefühlen von Einsamkeit (60\%) und Unsicherheit (59\%), Dingen, die einen beunruhigt haben, die einem aber sonst nichts ausmachten (57\%), Niedergeschlagenheit (56\%) und Schlafproblemen (50\% selten/nie und 33\% manchmal) verbunden gewesen wäre. Während eine Mehrheit der Befragten angab, dass Konzentrationsschwierigkeiten selten bzw. nie vorgekommen waren (52\%), meinten 36\%, dass sie oft bzw. sehr oft dieses Problem hatten. Eine neuerliche Auseinandersetzung mit schwierigen Ereignissen aus der eigenen Vergangenheit wurde ebenfalls von einer Mehrheit als selten angeführt (48\%), bei 23\% der Befragten war dies manchmal der Fall und bei $28 \%$ oft 
bzw. sehr oft. Eine knappe Mehrheit äußerte selten Gefühle von Angst (44\% selten/nie, $17 \%$ manchmal und $36 \%$ oft $/ \mathrm{sehr}$ oft) und genereller Anstrengung ( $40 \%$ selten bzw. nie, $28 \%$ manchmal und $31 \%$ oft $/$ sehr oft).

Wie auch in der Online-Erhebung waren Sorgen und Ängste um Angehörige bei den Befragten der qualitativen Interviews besonders präsent. Die Ängste der Respondent/ inn/en umfassten dabei überwiegend gesundheitliche und finanzielle Probleme, welche die Pandemie bei der Familie im Herkunftsland hervorrufen könnte.

Weiters wurde an die Verpflichtungen gedacht, die jede/r Einzelne hatte, falls es zu medizinischen Not- oder sogar zu Todesfällen in der Familie gekommen wäre. Aufgrund der Unmöglichkeit zu reisen, aber auch aufgrund der schwierigen Situationen, die durch den Jobsverlust in Österreich entstand, sahen sich manche Respondent/inn/en nicht in der Lage, adäquate Hilfe für Familienmitglieder leisten zu können.

Abb. 3.4.2: Emotionale und psychische Folgewirkungen der Coronakrise (Herkunft Syrien)

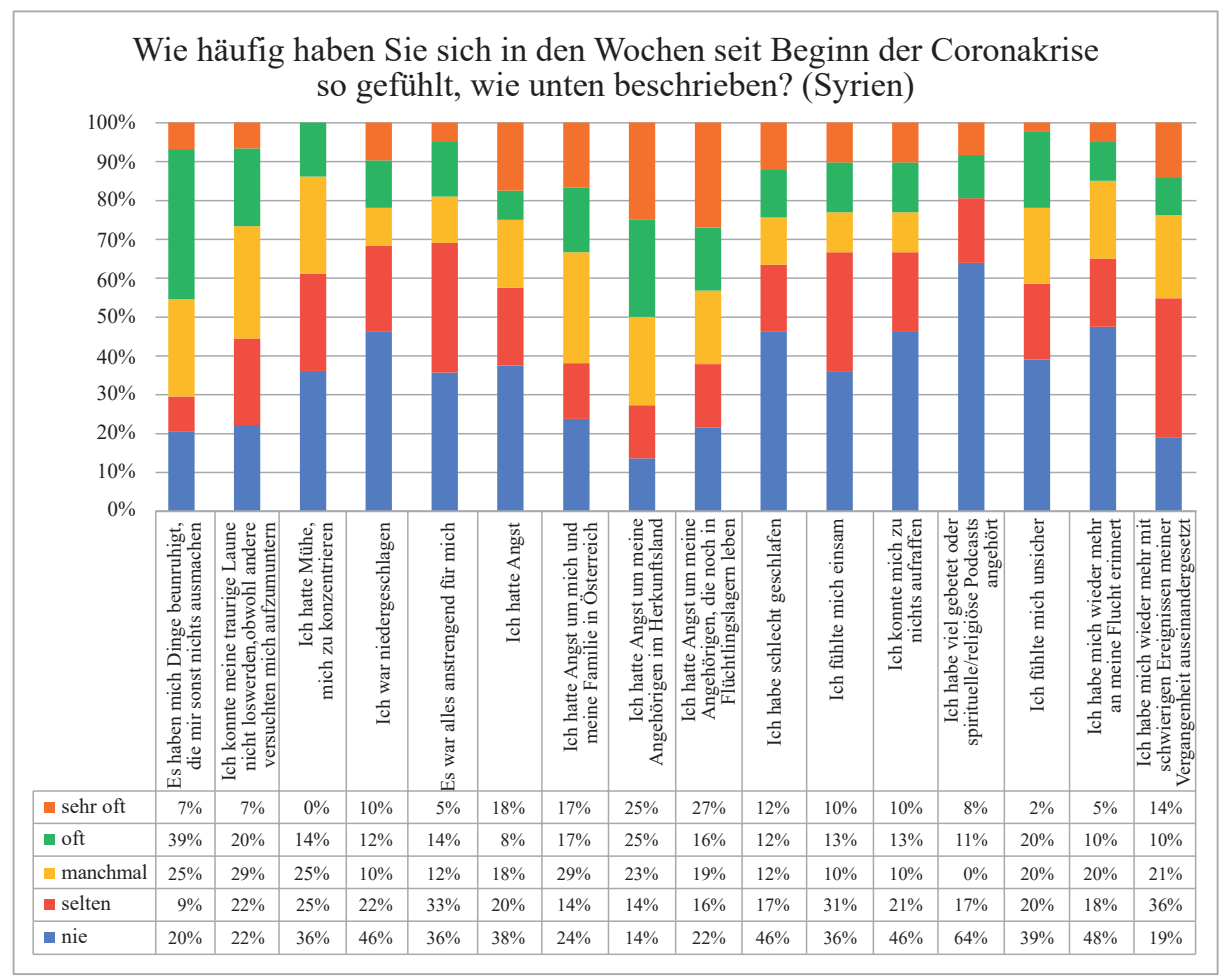

Quelle: eigene Erhebung 2020. 
Des Weiteren ging aus den narrativen Interviews hervor, dass die transnational verstreuten Familien versuchten, einander gegenseitig nicht allzu sehr zu verunsichern. Falls es zu Krankheitsfällen kam, wurden diese entweder heruntergespielt oder gar nicht erwähnt, um die Besorgnis unter- und umeinander nicht noch mehr zu verstärken.

Für manche der Befragten und der Expert/inn/en rief die Corona-Krise einen Modus hervor, den sie in den letzten Jahren bereits durchgemacht hatten. Wie zu jener Zeit, als sie auf der Flucht waren, dabei Polizei- und Staatsgewalt erfahren haben, von manchen dieser Erfahrungen schwer traumatisiert waren, fühlte sich die CoronaKrise als eine Weiterführung dieses Ausnahmezustandes an. Manche zeigten besondere Resilienz, andere hingegen hatten nicht mehr die Kraft, Angebote die sie zuvor wahrgenommen hatten, wie beispielsweise die Teilnahme an Sprachcafés, Spieleabenden etc. weiterzuführen oder nach dem Lockdown wiederaufzunehmen.

Wie in der Subgruppe Afghanistan, gaben auch Syrer/innen am öftesten an, Angst um Angehörige im Herkunftsland gehabt zu haben (50\%) (vgl. Abb. 3.4.2). Die Angst um Angehörige im Flüchtlingslager wurde von 43\% mit oft/sehr oft empfunden (19\% manchmal, 28\% selten/nie) und jene um die Familie in Österreich zu 34\% (29\% manchmal, 38\% selten oder nie). Anders als Afghan/inn/en haben Syrer/innen vermehrt Dinge beunruhigt, die ihnen sonst nichts ausmachten (46\% oft/sehr oft, 25\% manchmal, $29 \%$ selten/nie).

Mit Abstand am seltensten haben Syrer/innen gebetet und spirituelle/religiöse Podcasts gehört (81\%). Des Weiteren hat eine Mehrheit des syrischen Teilsamples angegeben selten (bzw. nie) allgemeine Anstrengung zu verspüren (69\%), niedergeschlagen zu sein (68\%), sich einsam zu fühlen (67\%), unmotiviert zu sein $(67 \%)$, erneute Erinnerungen an die eigene Flucht zu haben (66\%), schlecht geschlafen zu haben (63\%), Konzentrationsschwierigkeiten zu haben (61\%), sich unsicher $(59 \%)$ oder ängstlich (58\%) zu fühlen, sowie sich erneut mit schwierigen Ereignissen aus der eigenen Vergangenheit auseinandergesetzt zu haben (55\%). Auch gaben 44\% der Syrer/innen an, dass sie selten (bzw. nie) traurige Laune hatten, die sie nicht loswerden konnten. Bei 29\% war dies manchmal der Fall und bei 28\% sehr oft/oft.

Bei beiden Gruppen hat vor allem die Situation in den Herkunfts- und Transitländern Angst um Angehörige evoziert, die bei weitem die Angst im Kontext der Situation in Österreich überwog. Hier waren es vor allem die Verschärfung des politischen Ausnahmezustandes durch COVID-19, die mangelnde medizinische Infrastruktur und die geringen Vorsorgemaßnahmen, die zu den Maßnahmenkatalogen in Mitteleuropa in einem krassem Gegensatz standen. Aus den qualitativen Befragungen war ersichtlich, dass die eigene Angst sich nicht zu sehr auf die medizinische Versorgung in Notfällen oder die eigene ökonomische Situation fokussierte, sondern viel stärker auf die eigenen Zukunftspläne, die durch die Pandemie beeinträchtigt waren (Familiennachzug, Ausbildung, Verbesserung der Sprachkenntnisse). 


\subsection{Coping-Strategien}

Abb. 3.5: Coping-Strategien zur Bewältigung der psychischen Belastungen (gesamt)

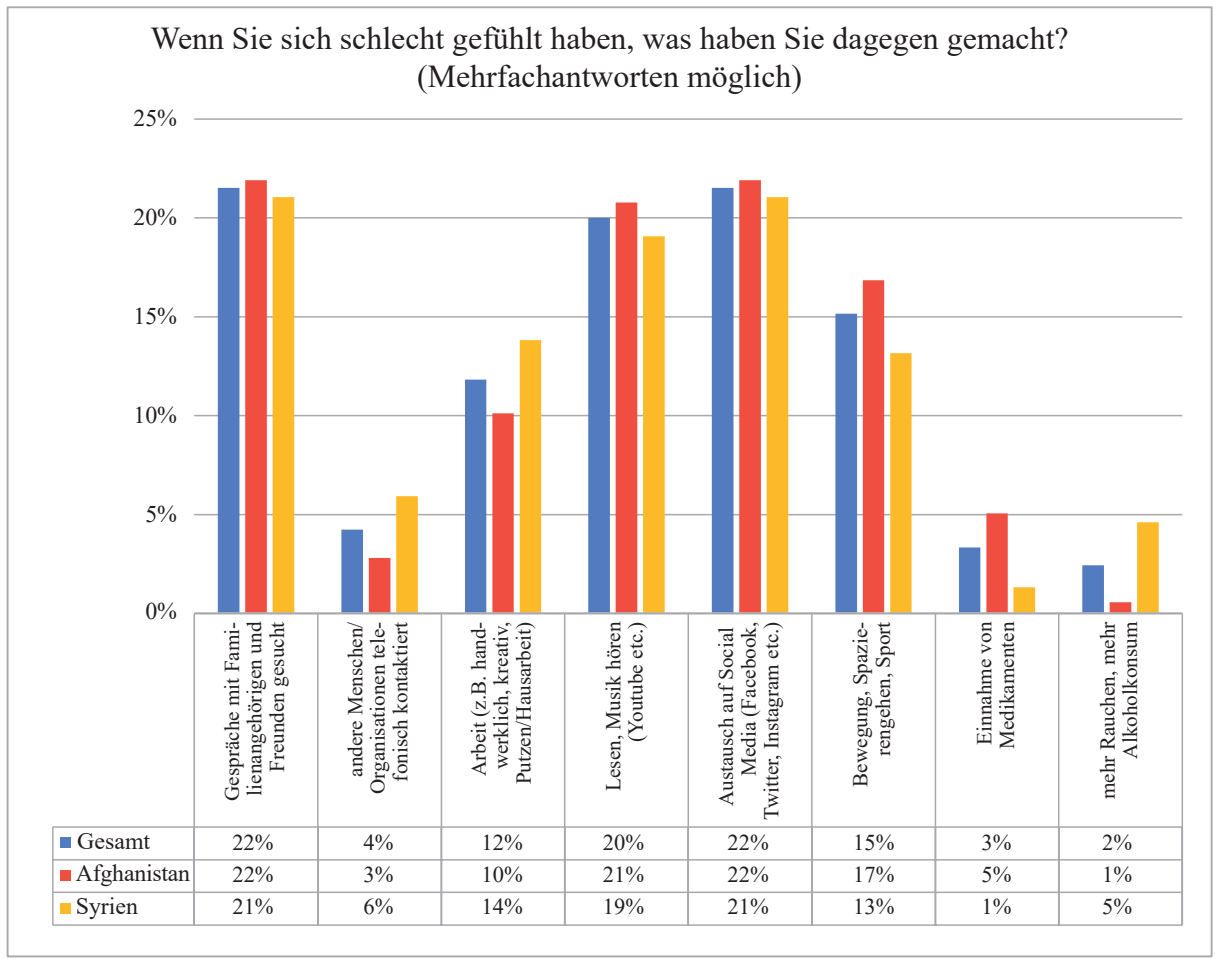

Quelle: eigene Erhebung 2020.

Da durch die unsichere Pandemie-Situation zahlreiche negative Gefühle auftraten, wurden Afghan/inn/en und Syrer/innen dazu befragt mittels welcher Coping-Strategien sie diesen schlechten Gefühlen entgegenwirkten (Mehrfachantworten waren möglich). Wie Abb. 3.5. veranschaulicht, haben die meisten Gespräche mit Familienangehörigen und Freund/inn/en (22\%) sowie den Austausch in den Sozialen Medien (22\%) gesucht, wenn sie sich schlecht fühlten. Der soziale Austausch, ob physisch bzw. virtuell, erwies sich somit auch als wichtigster Faktor zur Unterstützung des persönlichen Wohlbefindens in Zeiten der Corona-Pandemie. Auch Lesen und Musikhören war für 20\% der Befragten (Afghanistan 21\% und Syrien 19\%) eine wichtige Coping-Strategie. In den mittleren Wichtigkeitsbereich fielen Arbeit (handwerkliche, kreative oder Hausarbeit) und Bewegung (Spazierengehen und Sport). Bei beiden Kategorien zeigten sich minimale Unterschiede zwischen den beiden Herkunftsgruppen. Während Befragte aus Afghanistan vermehrt Bewegung machten (17\%; Syrer/innen 13\%), gingen Syrer/innen 
eher handwerklicher bzw. kreativer Arbeit nach (Syrer/innen 14\%; Afghan/inn/en 10\%). Weniger relevant war das telefonische Kontaktieren anderer Menschen und Organisationen (Afghanistan 3\%, Syrien 6\%), der Konsum von Alkohol sowie Rauchen (Afghanistan 1\%, Syrien 5\%) und die Einnahme von Medikamenten (Afghanistan 5\%, Syrien $1 \%)$. Hier zeigt sich, dass im vorliegenden Sample ein kleiner Prozentsatz der afghanischen Befragten in Zeiten der Krise eher zur Medikamenteneinnahme neigt, während syrische Befragte stärker zu Alkohol und zum Rauchen tendierten. Dies kann jedoch nicht als repräsentativ angesehen werden.

Ein vertiefender Einblick in individuelle Coping-Strategien gegen negative Gefühlen aufgrund der Pandemie-Situation war in den qualitativen Interviews möglich. Hier sprachen afghanische Befragte von der Wichtigkeit der Art des Zeitvertreibs, die den eigenen Gefühlen von Verunsicherung und Überforderung entgegenwirken sollte. Wie in der Online-Befragung, so dominierten auch bei den persönlichen Interviews folgende Tätigkeiten: Bewegung und Beschäftigung im Freien, Lesen und Serienschauen, Lernen (z.B. Deutsch, Unterrichtsgegenstände usw.) und vor allem das Kontaktieren und Gespräche führen mit Freund/inn/en.

Manche haben ganz bewusst gegen Gefühle von Ohnmacht und Angst angekämpft, und versuchten sich „Lichtblicke“ zu erarbeiten. Manchen ist es gut gelungen neue Coping-Mechanismen aufzubauen oder diese zu vestärken. Einige Familien haben auch versucht, durch den Besuch von Einrichtungen im Freien (beispielsweise Urban

Abb. 3.6: Geschlechtsspezifische Unterschiede der Coping-Strategien (gesamt)

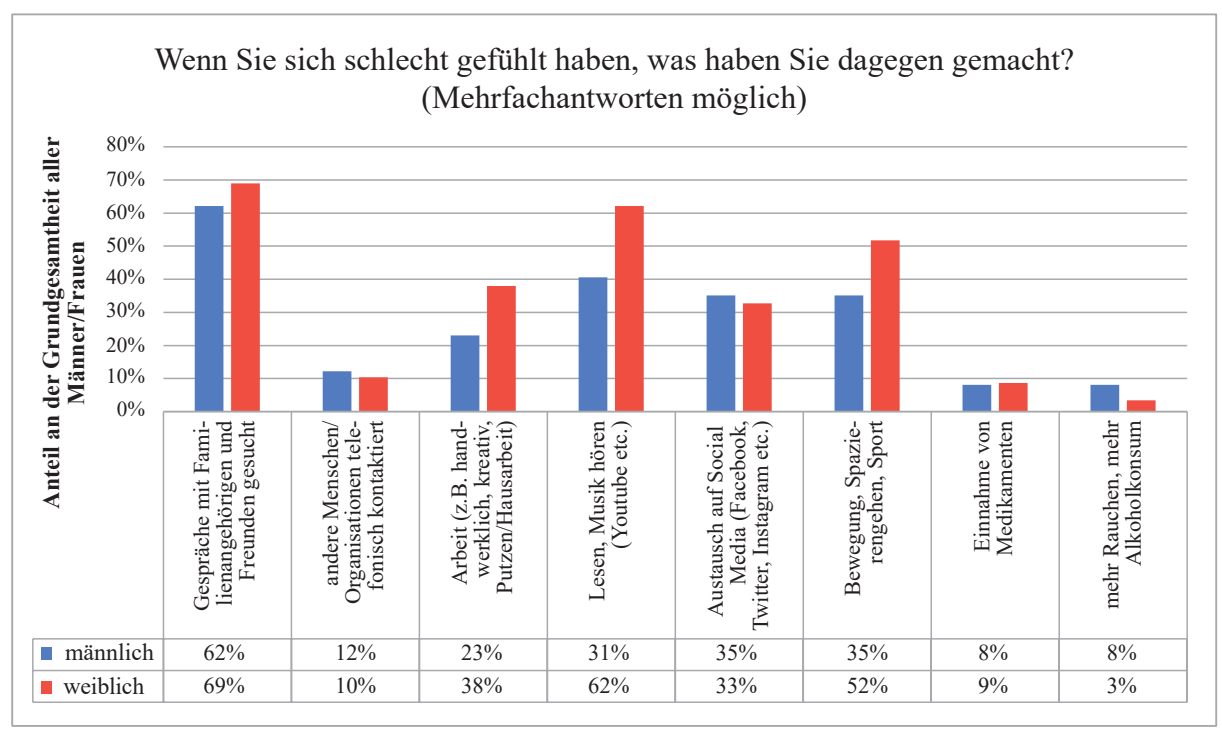

Quelle: eigene Erhebung 2020. 
Gardening), gemeinsame lange Spaziergänge, die sie vorher noch nicht unternahmen, der Situation zu begegnen oder sich ganz bewusst mit der deutschen Sprache auseinanderzusetzen.

Doch nicht jede/r wusste sich in dieser Krisenzeit zu helfen. Das Wegbrechen der gewohnten Alltagsstruktur und der über die letzten Jahre mühsam aufgebauten sozialen Netzwerke bedingte auch persönliche Überforderung. Bei manchen löste dies depressives Stimmungslagen aus, welche mithilfe von Genussmitteln (Alkohol, vermehrter Zigarettenkonsum) zu kompensieren versucht wurden.

Im Rahmen der Aktivitäten und Strategien gegen schlechte Befindlichkeit lagen in den meisten Antwortkategorien die Frauen beträchtlich vor den Männern (vgl. Abb. 3.6). Am häufigsten wurden von beiden Geschlechtern Gespräche mit Familienangehörigen oder Freund/inn/en gesucht (Frauen 68\% und Männer 62\%), beim Lesen bzw. Hören von Musik lagen die Frauen mit $62 \%$ mit großem Abstand vor den Männern (41\%), aber ebenso in der Kategorie Bewegung/Spazierengehen/Sport (Frauen 52\%, Männer 35\%) sowie bei handwerklicher bzw. Hausarbeit (Frauen 38\%, Männer 23\%).

Nahezu im selben Ausmaß haben beide Geschlechter Social Media benutzt bzw. zu Medikamenten gegriffen. Ein augenfälliger genderbezogener Unterschied manifestiert sich hinsichtlich der Kompensation schlechter Befindlichkeit durch gesteigerten Alkoholkonsum, der bei den Männern erheblich höher war ( $8 \%$ gegenüber 3\% bei den Frauen), um 1\% höher war bei den weiblichen Geflüchteten die Einnahme von Medikamenten.

In dem Versuch diese Befindlichkeiten auch vor dem Hintergrund unterschiedlicher Bildungsabschlüsse anzusehen, haben sich folgende Erkenntnisse ergeben. Die konkrete Frage war ,Wenn Sie sich schlecht gefühlt haben, was haben Sie dagegen gemacht?" und im Folgenden wird das bivariat ausgewertet dargestellt:

Tabelle 5 bezieht sich auf die Grundgesamtheit der abgegebenen Antworten. Strategien zur Minderung der Belastungen des Lockdowns zeigten sich in einem gewissen Ausmaß durch das Bildungsniveau beeinflusst. Gespräche mit Familienangehörigen und Freund/inn/en wurden zu $80 \%$ von Geflüchteten ohne Bildungsabschluss sowie Befragten mit Sekundärabschlüssen (73\%) gesucht. Weniger präsent waren hier die beiden anderen Bildungsgruppen. Der deutlichste Unterschied zeigte sich hinsichtlich der Nutzung von Social Media: Diese wurden zu 54\% von der Gruppe mit dem höchsten Bildungslevel genutzt, waren bei Befragten ohne Schulabschluss aber nicht relevant. Auch in der Subgruppe mit sekundären Abschlüssen war die Nutzung eher bescheiden. Lesen/Musik/Youtube waren in allen Bildungsgruppen durchwegs beliebte Ablenkungsstrategien. Zwar lagen hier ebenfalls die Befragten mit tertiären Anschlüssen (54\%) vorne, danach kamen aber die Befragten ohne Schulabschluss (50\%, wobei diese in erster Linie Youtube-Videos konsumierten) sowie mit geringen Unterschieden auch die beiden mittleren Bildungslevels. Hinsichtlich der sportlichen Betätigung waren alle 
Tab. 5: Coping-Strategien nach Bildungsniveau (gesamt)

\begin{tabular}{|l|r|r|r|r|}
\hline & $\begin{array}{c}\text { kein } \\
\text { Bildungs- } \\
\text { abschluss }\end{array}$ & $\begin{array}{c}\text { primärer } \\
\text { Bildungs- } \\
\text { abschluss }\end{array}$ & $\begin{array}{c}\text { sekundärer } \\
\text { Bildungs- } \\
\text { abschluss }\end{array}$ & $\begin{array}{c}\text { tertiärer } \\
\text { Bildungs- } \\
\text { abschluss }\end{array}$ \\
\hline $\begin{array}{l}\text { Gespräche mit Familienangehörigen } \\
\text { und Freund/inn/en gesucht }\end{array}$ & $80 \%$ & $56 \%$ & $73 \%$ & $59 \%$ \\
\hline $\begin{array}{l}\text { andere Menschen/Organisa-tionen } \\
\text { telefonisch kontaktiert }\end{array}$ & $20 \%$ & $3 \%$ & $6 \%$ & $24 \%$ \\
\hline $\begin{array}{l}\text { Arbeit (z.B. handwerklich, kreativ, } \\
\text { Putzen/Hausarbeit) }\end{array}$ & $30 \%$ & $34 \%$ & $23 \%$ & $38 \%$ \\
\hline $\begin{array}{l}\text { Lesen, Musik hören (Youtube etc.) } \\
\text { Austausch auf Social Media } \\
\text { (Facebook, Twitter, Instagram etc.) }\end{array}$ & $50 \%$ & $47 \%$ & $48 \%$ & $54 \%$ \\
\hline Bewegung, Spazierengehen, Sport & $30 \%$ & $47 \%$ & $35 \%$ & $49 \%$ \\
\hline Einnahme von Medikamenten & $0 \%$ & $6 \%$ & $6 \%$ & $8 \%$ \\
\hline $\begin{array}{l}\text { mehr Rauchen, mehr } \\
\text { Alkoholkonsum }\end{array}$ & $0 \%$ & $9 \%$ & $2 \%$ & $11 \%$ \\
\hline
\end{tabular}

Quelle: eigene Erhebung 2020.

aktiv. Hier lagen Respondent/inn/en mit Tertiärbildung ebenfalls an der Spitze (49\%), gefolgt von den Befragten mit primären Abschlüssen und danach die anderen beiden Bildungslevels. Auch hinsichtlich der Negativfolgen wie der Einnahme von Medikamenten sowie dem Rauchen/Alkoholkonsum waren die höchsten Werte bei Personen mit Universitätsausbildung zu konstatieren, in Bezug auf Rauchen/Alkohol gefolgt von Befragten mit primärer Bildung. 


\section{GeSUNDHEIT}

\subsection{Selbsteinschätzungen und Stressfaktoren}

Abb. 4.1: Individuelle Einschätzung des Gesundheitszustands (gesamt und nach Herkunft)

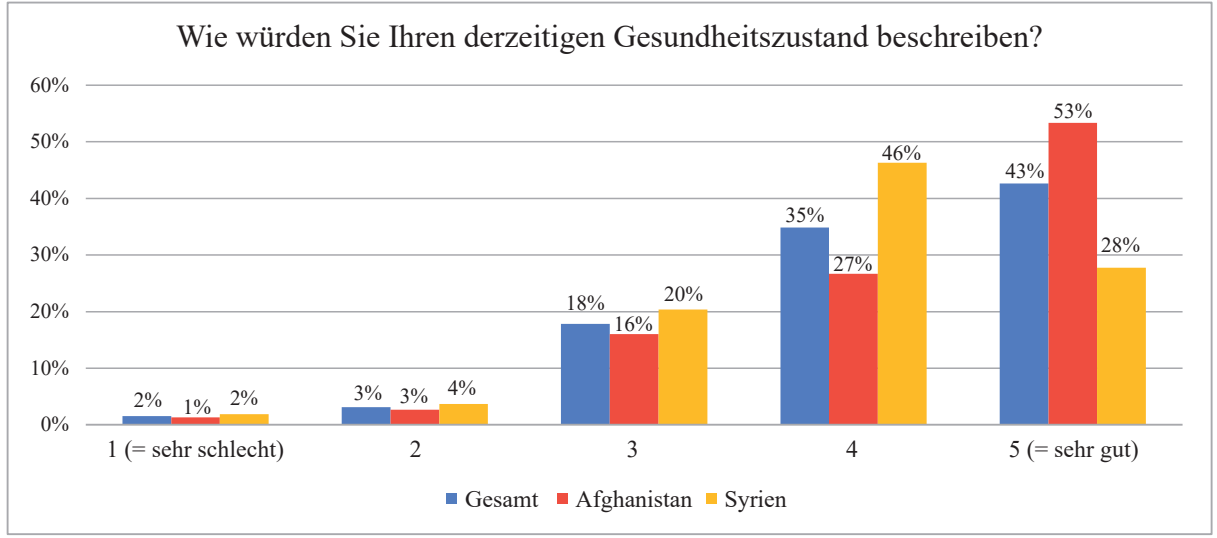

Quelle: eigene Erhebung 2020.

43\% der Respondent/inn/en haben den eigenen gesundheitlichen Zustand als sehr gut klassifiziert, weitere 35\% gaben gute Bewertungen ab (vgl. Abb. 4.1). Sehr schlecht bzw. schlecht beurteilten nur 5\% die eigene Gesundheit. Hierbei divergieren die Einschätzungen der beiden Analysegruppen allerdings beträchtlich. Während bei den Afghan/inn/en 53\% eine sehr gute Beurteilung ihrer Gesundheit abgaben, waren es bei den syrischen Befragten nur 43\%. Letztere entschieden sich öfter (46\%) für eine Bewertung als gut (Afghan/inn/en 27\%). Zu einem etwas höheren Anteil (6\%) als bei den Afghan/inn/en (4\%) wurde seitens der Befragten aus Syrien die eigene Gesundheit als eher schlecht oder sehr schlecht bewertet. Diesbezüglich interessant wären Vergleichswerte aus der Zeit vor der Coronakrise, die aber leider nicht verfügbar sind. Diese Werte wären aber notwendig, um die gesundheitlichen Auswirkungen von COVID-19 besser im Detail abschätzen zu können.

Hinsichtlich der Veränderungen des eigenen Gesundheitszustandes infolge der Pandemie (vgl. Abb. 4.2) zeigt sich, dass jeweils 23\% in beiden Communities eine Veränderung festgestellt haben. Auffällig hoch ist allerdings mit 15\% (Syrien) sowie 17\% (Afghanistan) der Anteil derjenigen, welche hier „weiß nicht“ angekreuzt haben. 
Abb. 4.2: Einschätzung der Veränderungen des eigenen Gesundheitszustandes während der Coronakrise (gesamt)

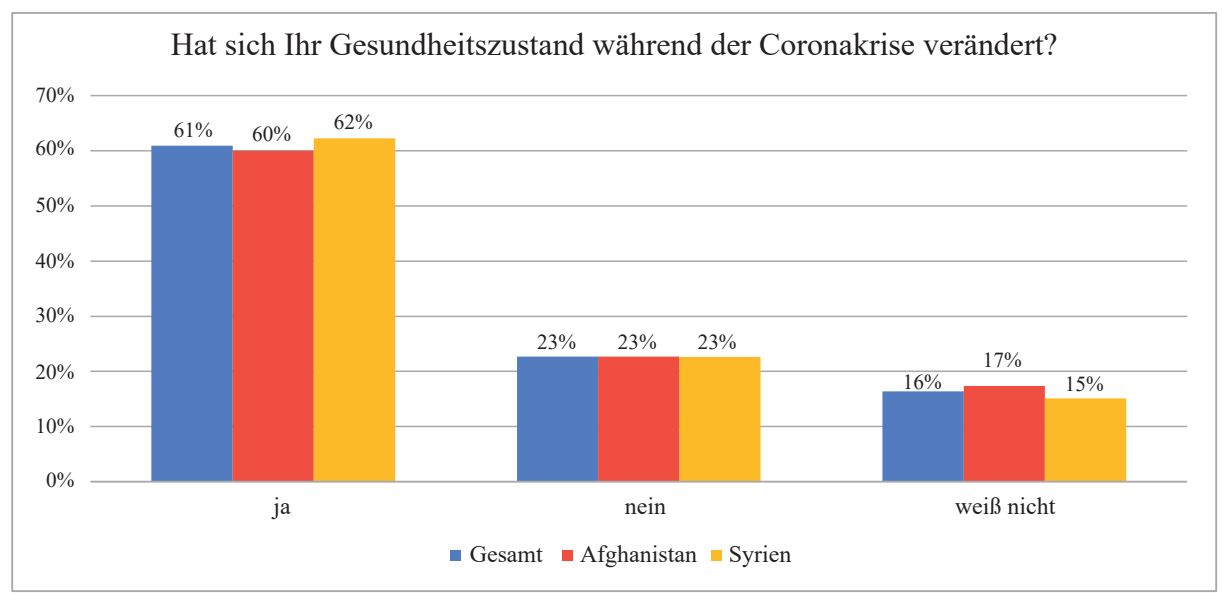

Quelle: eigene Erhebung 2020.

Abb. 4.3: Art der Veränderung des Gesundheitszustandes (gesamt)

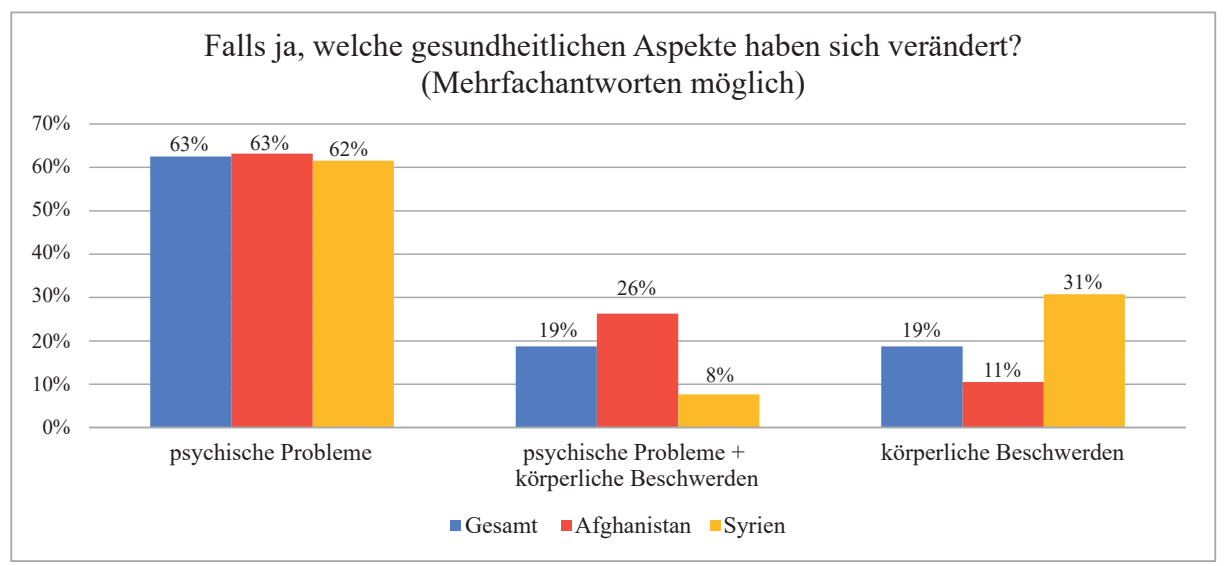

Quelle: eigene Erhebung 2020.

Um Aufschluss über die Spezifika der gesundheitlichen Veränderungen zu gewinnen, wurden diese ebenfalls abgefragt (vgl. Abb. 4.3). Bezogen auf die Grundgesamtheit haben $63 \%$ angegeben, psychische Beschwerden verspürt zu haben, jeweils 19\% hatten als Konsequenz der Pandemie organische bzw. eine Kombination aus organischen und psychischen Problemen. Aufschlussreich ist, dass wesentlich höhere Anteile der Befragten über psychische als über physische Beschwerden klagten, nämlich 
$63 \%$ der afghanischen und $62 \%$ der syrischen Befragten. Hinsichtlich der physischen Beschwerden besteht ein erheblicher Unterschied zwischen den Syrer/inne/n, die zu $31 \%$ auch physische Probleme angaben, während dies nur bei 11\% der Afghan/inn/en der Fall war. Dafür waren letztere erheblich öfter (26\%) von einer Kombination psychischer und organischer Beschwerden betroffen (die Syrer/innen nur zu 8\%).

Psychische Probleme verschiedenster Art, von Depressionen über Angststörungen etc., wurden sowohl von den interviewten Expert/inn/en als auch den Interviewpartner/ inne/n als weit verbreitete Corona-Folgewirkungen in allen Altersgruppen der Communities beschrieben. Die Verschränkung von physischen und psychischen Problemen wurde von manchen ebenfalls thematisiert.

In diesem wie auch in weiteren Bereichen ist die besondere und weit gefasste Rolle von Mediziner/inne/n offensichtlich. Sie sind für viele die ersten Ansprechpartner/innen bei physischen, psychischen und auch strukturellen Problemen. Das Vertrauen in Einrichtungen, die psychologische Beratungen anbieten, war für manche Befragte, die sich noch im Asylverfahren befanden, nicht gegeben. Manche fürchteten, dass Informationen über sie an das Bundesamt für Fremdenwesen und Asyl weitergegeben würden. Einige der Expert/inn/en meinten auch, dass das Bewusstsein für und das Wissen um Symptome psychischer Erkrankungen bei Betroffenen nicht immer vorhanden war. Besondere Stressfaktoren, die sich auf die Gesundheit auswirkten, waren die soziale Isolation und unterschiedliche Ängste (Zukunftsängste, Verlust des Arbeitsplatzes).Vorhandene belastende Konstellationen (Sorgen um Ausbildung, Arbeit, Asylstatus etc.) wurden durch die Pandemie noch verstärkt. Manche der Befragten, die alleine wohnten, hatten während des ersten Lockdowns keine sozialen Kontakte. Sie waren alleine mit ihren Problemen, ohne Austausch, Abwechslung und Aufgaben, was bestehende Depressionen erheblich verstärkte. Manche wollten auch bewusst niemanden in ihrem Bekanntenkreis mit ihren Problemen konfrontieren, da sie befürchteten, dass dies eine zu große Herausforderung für Gesprächspartner/innen sein könnte, die selber auch an psychischen Belastungen oder sogar Depressionen litten. Für manche bedeutete das Physical Distancing eine Belastung der sozialen Beziehungen, da distantes soziales Verhalten in den Communities generell als unmoralisch bewertet wird. Wenn in dieser Zeit dann noch weitere zusätzliche Belastungen hinzukamen, wie beispielsweise ein Autounfall, eine zusätzliche Erkrankung oder die überbordende Belastung vor allem für Mütter im Homeschooling, gelangten die Befragten an ihre Grenzen. Von manchen Expert/inn/en wurde auch häusliche Gewalt problematisiert. Der Ausfall langsam aufgebauter sozialer Netzwerke, der Verlust der Tagesstruktur (durch Jobverlust, Kurzarbeit) oder zusätzliche strukturelle Probleme (z.B. Wohnsituation) haben zur Verschärfung der Situation beigetragen. Das heißt, dass sowohl strukturelle als auch juristische Probleme wie auch soziale Herausforderungen und Hürden zur Verschlechterung des subjektiven Gesundheitsempfindens beigetragen haben.

Wie schon dargestellt waren die ersten Ansprechpersonen oft Mediziner/innen, die diesbezüglich auf die Notwendigkeit multidisziplinärer Teams hingewiesen haben, um die Menschen ausreichend betreuen zu können. Ferner haben Mediziner/innen festgestellt, dass der generelle Trend während des ersten Lockdowns zur Vermeidung von 
Vorsorge- und Kontrolluntersuchungen (z.B. Mutter-Kind-Pass) langfristig negative Entwicklungen zur Folge hatte.

Die Akkumulation von psychischen Belastungen hatte in einigen Fällen sogar Re-Traumatisierungseffekte zur Folge, wozu es allerdings kaum aktuelle Grundlagenforschungen gibt. Expert/inn/en meinten, dass Menschen durch die Selbstisolation an Kriegserfahrungen erinnert wurden. Des Weiteren hatten die Informationen über die COVID-19-Situation in den Herkunftsländern, die Entwicklungen bei nicht geflüchteten Familienmitgliedern, die psychischen Probleme zusätzlich verstärkt.

In einigen Extremfällen akkumulierten sich die psychischen Probleme sogar zu einer regelrechten Coronaphobie. Diese konnte sich in fast schon übertriebenen Hygienemaßnahmen und Formen der Selbstisolation wie auch in der Kontrolle von Familienmitgliedern äußern.

Es fühlte sich exakt die Hälfte der Respondent/inn/en infolge der Pandemie in einem stärkeren Ausmaß gestresst, davon die Afghan/inn/en in einem höheren Ausmaß $(53 \%)$ als die Befragten aus Syrien (44\%). Auffällig ist in Bezug auf diese Frage auch der vor allem bei den Afghan/inn/en hohe Anteil jener (26\%), die hier unsicher waren und ,weiß nicht“" angegeben haben (bei den Syrer/inne/n nur 6\%). Eine Erklärungshypothese für diesen ausgeprägten Unterschied liegt wahrscheinlich im hohen Grad an Tabuisierung psychischer Beschwerden, Stresssymptomatiken und Depressionen in der afghanischen Gesellschaft allgemein und im Besonderen bei den Männern. Psychische Erkrankungen wirken stigmatisierend und werden in den Communities nach Möglichkeit verschwiegen. Nur selten wird ärztliche Hilfe gesucht bzw. angenommen.

Um Aufschluss über die Stressursachen zu gewinnen, wurden auch die Kausalfaktoren für den während der Pandemie empfundenen Stress abgefragt (vgl. Abb. 4.4). Dabei zeigen sich für das gesamte Sample zwei besonders häufig auftretende Stressoren, nämlich der Verlust von Sozialkontakten (16\%) sowie finanzielle Probleme (15\%). Danach folgen mit einigem Abstand die Unklarheit über die Situation von Angehörigen (11\%) und Probleme im Rahmen der Ausbildung bzw. des Studiums (10\%). Die anderen im Rahmen dieser Frage vorgegebenen Stressfaktoren waren weniger relevant, z.B. Arbeitsplatzverlust sowie schulische Probleme der Kinder jeweils mit 6\%. Familiäre Konflikte rangierten mit 7\% ebenfalls eher hinten, obwohl diese seitens der Expert/inn/ en als häufig aufgetretenes Problem verbalisiert wurden. Geringe Bedeutung hatte mit 4\% auch die Unmöglichkeit des Familiennachzugs.

Die Unterschiede zwischen den beiden untersuchten Gruppen sind ausgeprägt (vgl. Abb. 4.4). Befragte aus Afghanistan litten primär unter finanziellen Problemen $(17 \%)$, dem Verlust der Sozialkontakte (15\%), an ausbildungsbezogenen Problemen (13\%) sowie jeweils zu 11\% an familiären Konflikten und der Unklarheit über die Situation von Familienangehörigen. Am wenigsten betroffen waren sie von Problemen hinsichtlich der Kinderbetreuung (hoher Singleanteil!), der Schließung des eigenen Geschäftes (kaum Selbständige!) oder dem Verlust von Unterstützungsangeboten. 
Abb. 4.4: Welche Stressfaktoren haben sich durch die Coronakrise verschärft? (gesamt und nach Herkunft)

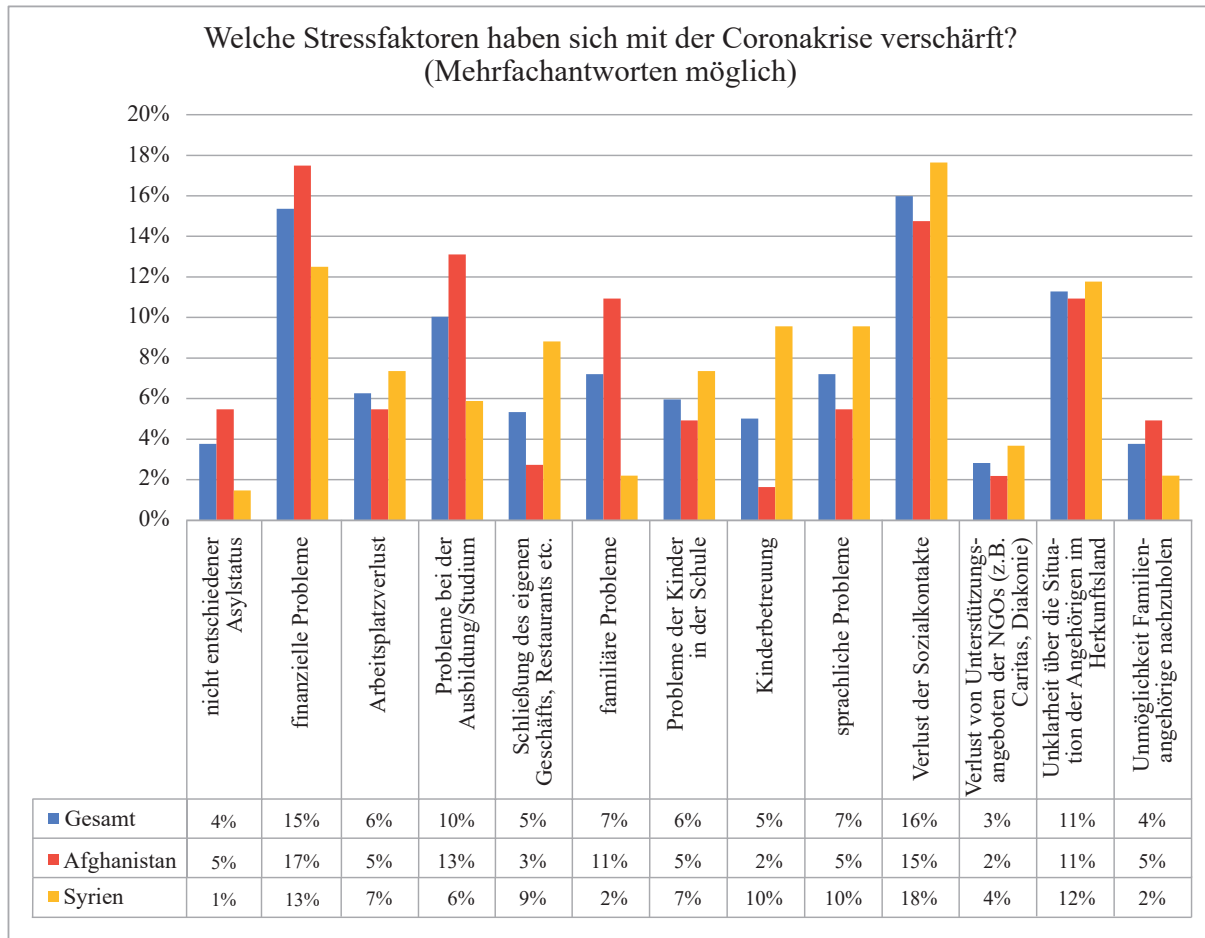

Quelle: eigene Erhebung 2020.

Bei den syrischen Respondent/inn/en (vgl. Abb. 4.4) lagen die Hauptursachen für Stress während der Coronakrise in erster Linie am Verlust der sozialen Kontakte (18\%), an finanziellen Problemen (13\%) sowie der Unklarheit über die Situation von Angehörigen (12\%). Deutlich häufiger als bei den Afghan/inn/en wurden seitens der Syrer/innen zudem genannt: Probleme der Kinderbetreuung und sprachliche Barrieren (jeweils zu 10\%), die Schließung des eigenen Unternehmens (9\%) und schulische Probleme der Kinder (7\%). Mit nur 2\% waren familiäre Probleme trotz des höheren Anteils von Ehepaaren und Familien mit Kindern bemerkenswerterweise weit weniger wichtig als bei den Afghan/inn/en, ebenfalls nur 2\% haben die Unmöglichkeit des Familiennachzugs als Stressfaktor angegeben.

Die narrativen Interviews mit afghanischen Befragten und Expert/inn/en aus den NGOs bestätigten die Resultate der Online-Befragung. Sorgen um den Job, Angst vor Arbeitslosigkeit und den Herausforderungen einen neuen Job finden zu müssen, Unsicherheit angesichts der sich verschärfenden Situation auf dem Wiener Wohnungsmarkt und familiäre Probleme bildeten bei vielen Befragten eine komplexe Gemengelage 
an Belastungen. Hinzu kamen noch ungenügende Deutschkenntnisse und Bildungsdefizite bei bestimmten Herkunftsgruppen. Die psychischen Belastungen wirkten bei manchen insbesondere auf die Familiensituation, die sich durch zusätzlichen Betreuungsbedarf für Ältere oder Kinder noch weiter verschärfte.

Aus der Sicht von von Betreuer/inne/n in NGOs wirkten folgende Faktoren noch weiter verschärfend: Der Wegfall bzw. die Einschränkung von Beratungsangeboten, wodurch manche Klient/inn/en niemanden mehr hatten, der ihnen Nachrichten von Ämtern und Behörden hätten übersetzen können und weiters das Unwissen zu Beginn der Pandemie, wo man in Konflikt- oder Notfällen Hilfe holen konnte. Die Betreuung der Kinder im Homeschooling war ein großer Stressfaktor und hat für manche Befragte das gesamte Familienleben bestimmt. Für die Betreuer/innen wie auch für die betroffenen Familien war die unterschiedliche Qualität der schulischen Hilfestellungen beim Homeschooling besonders auffallend. Für Eltern mit höheren Bildungsniveaus waren die im Vergleich zu jenen im Herkunftsland unterschiedlichen Lehr- und Lernsysteme an sich schwierig. Um die Kinder im Homeschooling unterstützen zu können, hat man auch oft Verwandte, die schon länger in Österreich lebten oder auch die Angebote von Hilfsorganisationen in Anspruch genommen.

Rund um den Stressfaktor Arbeit wurden Probleme bei der Kurzarbeit angegeben, wobei die Befragten in illegale Beschäftigungsverhältnisse gedrängt wurden (manche waren offiziell in Kurzarbeit, haben jedoch Vollzeit und mit unbezahlten Überstunden gearbeitet). Arbeitslosigkeit implizierte auch die Bewältigung nötiger Amtswege (AMS), für die manche zusätzliche Unterstützung benötigten. Der Verlust der Arbeit bedeutete auch, dass die Ziele, die man für sich und die Familie gesteckt hatte, nämlich die frühestmögliche Beantragung der Staatsbügerschaft (wofür durchgehende Beschäftigungsverhältnisse besonders wichtig sind), in weitere Ferne rückten.

Die Sorge um im Ausland lebende Familienangehörige und deren Gesundheitszustand in Anbetracht der globalen Pandemie machte vielen befragten Afghan/inn/en besonders zu schaffen. Sie verspürten in diesem Zusammenhang sogar mehr Angst als davor selber zu erkranken. Auch der geplante Nachzug von Familienangehörigen war betroffen. Durch die Einschränkung der Reisefreiheit konnten Familienmitglieder, die in der Heimat oder in Transitländern festsaßen, nicht nach Österreich gelangen. Ferner war auch in den Transitländern die Unterstützung durch Ehrenamtliche nicht mehr in dem Ausmaß gegeben wie vor COVID-19.

Einzelne Interviewees haben angesichts steigender Infektionszahlen auch bereits im Spätsommer ihre steigende Angst vor einer zweiten Welle und weiteren Lockdowns bekundet. 


\subsection{Geschlechtsspezifische und berufliche Unterschiede in Bezug auf Stressfaktoren}

Die Auswertungen (vgl. Tab. 6) der Stressfaktoren in Bezug auf die Gendervariable zeigen, dass die Stressoren die beiden Geschlechter in unterschiedlichem Ausmaß betroffen haben. Von den Folgen im Zusammenhang mit dem Asylverfahren waren mehr Männer häufiger tangiert. Etwas mehr (17,2\% zu 13,5\%) machte Frauen jedoch Arbeitsplatzverlust zu schaffen und beträchtlich häufiger $(32,8 \% \mathrm{zu} 17,6 \%)$ hatten sie Probleme mit der laufenden Ausbildung bzw. dem Studium. Ein deutlicher Unterschied manifestiert sich auch bei den auf Kinder bezogenen Herausforderungen. So gaben 27,6\% der Frauen, aber nur 17,6\% der Männer schulische Probleme der Kinder als für sie zutreffend an, hinsichtlich der Betreuung der Kinder waren Unterschiede vorhanden jedoch weniger krass. Der Verlust von Sozialkontakten belastete die Frauen im Sample erheblich mehr (43,1\% gegenüber 35,1\% der Männer). Der Logik des Familiennachzugs inhärent ist, dass Männer beträchtlich stärker von der Unmöglichkeit in diesem Feld betroffen waren. Hinsichtlich der finanziellen Probleme waren die Unterschiede nicht sehr ausgeprägt, dasselbe galt hinsichtlich der Schließung von Unternehmen bzw. Geschäften. Auch familiäre Probleme wurden von beiden Geschlechtern fast gleichermaßen häufig angegeben.

Tab. 6: Geschlechtsspezifische Unterschiede in Bezug auf Stressfaktoren (gesamt)

\begin{tabular}{|c|c|c|c|}
\hline & männlich & weiblich & Gesamtergebnis \\
\hline \multicolumn{4}{|l|}{ Asylstatus } \\
\hline nicht zutreffend & $89,19 \%$ & $93,10 \%$ & $90,91 \%$ \\
\hline zutreffend & $10,81 \%$ & $6,90 \%$ & $9,09 \%$ \\
\hline Gesamtergebnis & $100,00 \%$ & $100,00 \%$ & $100,00 \%$ \\
\hline \multicolumn{4}{|c|}{ finanzielle Probleme } \\
\hline nicht zutreffend & $62,16 \%$ & $63,79 \%$ & $62,88 \%$ \\
\hline zutreffend & $37,84 \%$ & $36,21 \%$ & $37,12 \%$ \\
\hline Gesamtergebnis & $100,00 \%$ & $100,00 \%$ & $100,00 \%$ \\
\hline \multicolumn{4}{|c|}{ Arbeitsplatzverlust } \\
\hline nicht zutreffend & $86,49 \%$ & $82,76 \%$ & $84,85 \%$ \\
\hline zutreffend & $13,51 \%$ & $17,24 \%$ & $15,15 \%$ \\
\hline Gesamtergebnis & $100,00 \%$ & $100,00 \%$ & $100,00 \%$ \\
\hline \multicolumn{4}{|c|}{ Probleme bei der Ausbildung/Studium } \\
\hline nicht zutreffend & $82,43 \%$ & $67,24 \%$ & $75,76 \%$ \\
\hline zutreffend & $17,57 \%$ & $32,76 \%$ & $24,24 \%$ \\
\hline Gesamtergebnis & $100,00 \%$ & $100,00 \%$ & $100,00 \%$ \\
\hline
\end{tabular}




\begin{tabular}{|c|c|c|c|}
\hline & männlich & weiblich & Gesamtergebnis \\
\hline \multicolumn{4}{|c|}{ Schließung des eigenen Geschäfts, Restaurants etc. } \\
\hline nicht zutreffend & $87,84 \%$ & $86,21 \%$ & $87,12 \%$ \\
\hline zutreffend & $12,16 \%$ & $13,79 \%$ & $12,88 \%$ \\
\hline Gesamtergebnis & $100,00 \%$ & $100,00 \%$ & $100,00 \%$ \\
\hline \multicolumn{4}{|c|}{ familiäre Probleme } \\
\hline nicht zutreffend & $82,43 \%$ & $82,76 \%$ & $82,58 \%$ \\
\hline zutreffend & $17,57 \%$ & $17,24 \%$ & $17,42 \%$ \\
\hline Gesamtergebnis & $100,00 \%$ & $100,00 \%$ & $100,00 \%$ \\
\hline \multicolumn{4}{|c|}{ Probleme der Kinder in der Schule } \\
\hline nicht zutreffend & $82,43 \%$ & $72,41 \%$ & $78,03 \%$ \\
\hline zutreffend & $17,57 \%$ & $27,59 \%$ & $21,97 \%$ \\
\hline Gesamtergebnis & $100,00 \%$ & $100,00 \%$ & $100,00 \%$ \\
\hline \multicolumn{4}{|l|}{ Kinderbetreuung } \\
\hline nicht zutreffend & $89,19 \%$ & $86,21 \%$ & $87,88 \%$ \\
\hline zutreffend & $10,81 \%$ & $13,79 \%$ & $12,12 \%$ \\
\hline Gesamtergebnis & $100,00 \%$ & $100,00 \%$ & $100,00 \%$ \\
\hline \multicolumn{4}{|c|}{ sprachliche Probleme } \\
\hline nicht zutreffend & $82,43 \%$ & $82,76 \%$ & $82,58 \%$ \\
\hline zutreffend & $17,57 \%$ & $17,24 \%$ & $17,42 \%$ \\
\hline Gesamtergebnis & $100,00 \%$ & $100,00 \%$ & $100,00 \%$ \\
\hline \multicolumn{4}{|c|}{ Verlust der Sozialkontakte } \\
\hline nicht zutreffend & $64,86 \%$ & $56,90 \%$ & $61,36 \%$ \\
\hline zutreffend & $35,14 \%$ & $43,10 \%$ & $38,64 \%$ \\
\hline Gesamtergebnis & $100,00 \%$ & $100,00 \%$ & $100,00 \%$ \\
\hline \multicolumn{4}{|c|}{ Verlust von Unterstützungsangeboten der NGOs (z.B. Caritas, Diakonie) } \\
\hline nicht zutreffend & $91,89 \%$ & $94,83 \%$ & $93,18 \%$ \\
\hline zutreffend & $8,11 \%$ & $5,17 \%$ & $6,82 \%$ \\
\hline Gesamtergebnis & $100,00 \%$ & $100,00 \%$ & $100,00 \%$ \\
\hline \multicolumn{4}{|c|}{ Unklarheit über die Situation der Angehörigen im Herkunftsland } \\
\hline nicht zutreffend & $72,97 \%$ & $72,41 \%$ & $72,73 \%$ \\
\hline zutreffend & $27,03 \%$ & $27,59 \%$ & $27,27 \%$ \\
\hline Gesamtergebnis & $100,00 \%$ & $100,00 \%$ & $100,00 \%$ \\
\hline \multicolumn{4}{|c|}{ Unmöglichkeit Familienangehörige nachzuholen } \\
\hline nicht zutreffend & $86,49 \%$ & $94,83 \%$ & $90,15 \%$ \\
\hline zutreffend & $13,51 \%$ & $5,17 \%$ & $9,85 \%$ \\
\hline Gesamtergebnis & $100,00 \%$ & $100,00 \%$ & $100,00 \%$ \\
\hline
\end{tabular}

Quelle: eigene Erhebung 2020. 
Die unterschiedliche Wirksamkeit zahlreicher Stressfaktoren zeigte sich nicht nur in Bezug auf die Variablen Gender und Bildung, sondern auch in Abhängigkeit vom Beruf, welcher vor der Krise ausgeübt worden war. Am häufigsten betroffen von finanziellen Problemen waren Arbeitssuchende sowie Bezieher/innen der Mindestsicherung (zu je 50\%), daneben auch Angestellte zu fast 38\%. Arbeitsplatzverluste wurden vor allem von Selbständigen angegeben, Ausbildungsprobleme von Studierenden, Studierenden mit Teilzeitjobs sowie Schüler/inne/n. Geschäftsschließungen waren bei Selbständigen relevant, während familiäre Probleme ein Drittel der Arbeitssuchenden sowie $21 \%$ der Angestellten betrafen. Probleme der Kinder im schulischen Bereich wurden vor allem von Selbständigen, Mindestsicherungsempfänger/inne/n und Arbeitssuchenden angegeben, diese waren auch vom Verlust der Sozialkontakte am stärksten betroffen. Probleme mit der deutschen Sprache, exakter dem Ausfüllen von Formularen und Anträgen, haben in erster Linie Selbständige, Arbeitssuchende sowie Empfänger/innen der Mindestsicherung angegeben, die im Rahmen von Behördenkontakten damit am meisten konfrontiert waren. Der Verlust von Unterstützungsangeboten der NGOs, wie Caritas oder Diakonie, hat besonders Selbständige, Mindestsicherungsempfänger/innen und im Haushalt tätige Befragte betroffen. Von der Unmöglichkeit weitere Familienangehörige nachzuholen waren infolge finanzieller Limitationen in erster Linie Bezieher/ innen der bedarfsorientierten Mindestsicherung betroffen.

Es gibt erste Erhebungen bezüglich der finanziellen Belastungen durch die Pandemie bezogen auf Österreich und auf EU-Ebene. Statistik Austria berichtet von steigenden finanziellen Sorgen und der erheblichen materiellen Benachteiligung durch die Pandemie für alle in Österreich lebenden Personen. ${ }^{10}$

\subsection{Ansprechpersonen und -institutionen}

Dem hohen Ausmaß an Stress sowie physischen und psychischen Problemen generell Rechnung tragend wurde auch gefragt, welche Personen bzw. Institutionen zwecks Hilfestellung konsultiert wurden (vgl. Abb. 4.5). Am öftesten haben die Befragten Allgemeinmediziner/innen bzw. Fachärzt/inn/e/n mit muttersprachlichen Kompetenzen (35\%) oder Ärzt/inn/e/n aus demselben Herkunftsland wie die Befragten (zu 21\%) kontaktiert. Etwas weniger häufig haben sie sich an eine/n ,österreichischen“ praktischen Arzt/Ärztin um Hilfe gewandt (25\% oft bzw. sehr oft) sowie zu 20\% and eine/n österreichischen Facharzt/-ärztin. Österreichische Allgemeinmediziner/innen (13\%) bzw. Fachärzt/inn/e/n (19\%) wurden aber zumindest manchmal aufgesucht. Eine sehr wichtige Rolle als Unterstützer/innen spielten auch Freund/inn/e/n oder Bekannte aus der eigenen Herkunftscommunity. An diese wandten sich 13\% oft bzw. sehr oft und 26\% manchmal. Etwa gleich wichtig in der Bewältigung der COVID-19 bedingten gesundheitlichen Krisensituationen waren österreichische Freund/inn/e/n und Bekannte oder solche

10 Statistik Austria (2021), Pressemitteilung: 12.502-093/21, <https://www.statistik.at/web_ de/presse/125881.html>. 
Abb. 4.5: An wen wandten sich die Befragten bei gesundheitlichen Problemen, Depression oder Stress? (gesamt)

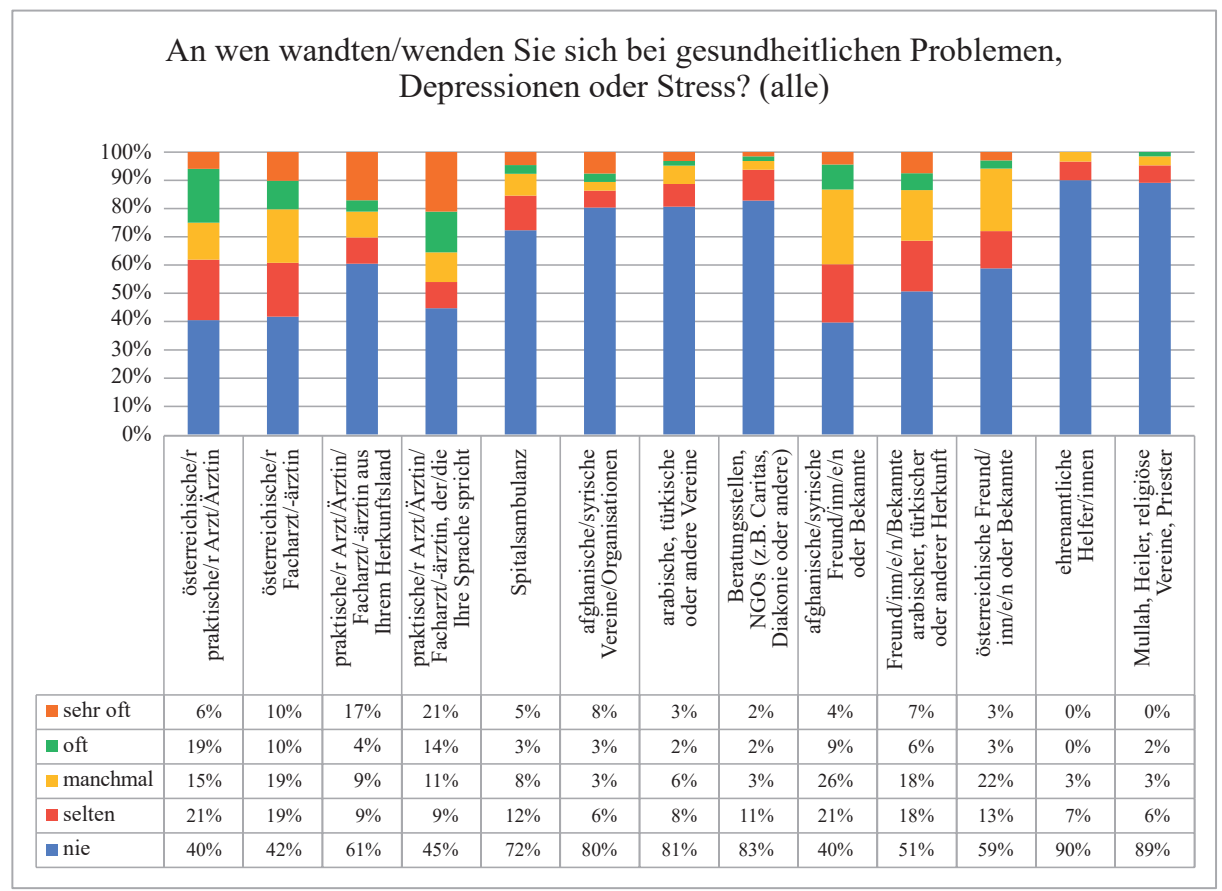

Quelle: eigene Erhebung 2020.

arabischer, türkischer oder anderer Herkunft. Am wenigsten wurden ehrenamtlich bei Vereinen oder NGOs tätige Personen ${ }^{11}$ (90\% nie) oder religiöse Funktionäre (Mullahs) ( $89 \%$ nie) um Hilfe gebeten. Beratungsstellen und NGOs haben $83 \%$ nie kontaktiert, arabische, türkische oder andere Vereine $81 \%$ niemals sowie afghanische/syrische Organisationen $80 \%$ nie. Zum besseren Verständnis dieser Resultate ist darauf hinzuweisen, dass in der Realität die Kagegorien „Ehrenamtliche“ und „Freund/inn/e/n“ oft ineinander verschwimmen. Zwischen ehrenamtlich engagierten Personen und den Geflüchteten können mehr oder weniger enge Freundschaften entstehen, die über eine professionelle Sozialbeziehung weit hinausgehen. Da im Rahmen dieser Frage eine klare Differenzierung nicht möglich war, ist davon auszugehen, dass viele Befragte „Ehrenamtliche“ als ,österreichische Freund/inn/e/n“ klassifizierten.

11 Hier könnte eine für viele Personen in den Communities typische Auffassung des „Ehrenamtes“ eine Rolle spielen. Viele Befragte klassifizieren ehrenamtliche Helfer/innen als Freund/ inn/e/n und können mit dem Terminus daher wenig anfangen. Die hohe faktische Bedeutung Ehrenamtlicher bei der Integration steht (vgl. Punz \& Schwarzbauer 2020) daher in einem offensichtlichen Widerspruch zu deren geringer Wichtigkeit in Kontext dieser Befragung. 
Interessant ist das hohe Maß an Vertrauen, der medizinischem Personal mit den jeweiligen muttersprachlichen Kompetenzen entgegengebracht wird. Dies muss nicht primär jemand aus der eigenen Herkunftscommunity sein, sondern eine Person mit den jeweiligen Sprachkompetenzen. Dies ist ein wesentlicher Faktor, der auch für den Ausbau von „Distance translating“-Einrichtungen spricht, wie auch für das Vertrauen, das in das österreichische Gesundheitssystem gelegt wird. Die einzige Hürde scheinen die muttersprachlichen Kompetenzen zu sein, die auch über andere Wege (z.B. Telefondolmetscherdienste) angeboten werden könnten.

Abb. 4.5.1: An wen wandten sich die Befragten bei gesundheitlichen Problemen, Depression oder Stress? (Herkunft Afghanistan)

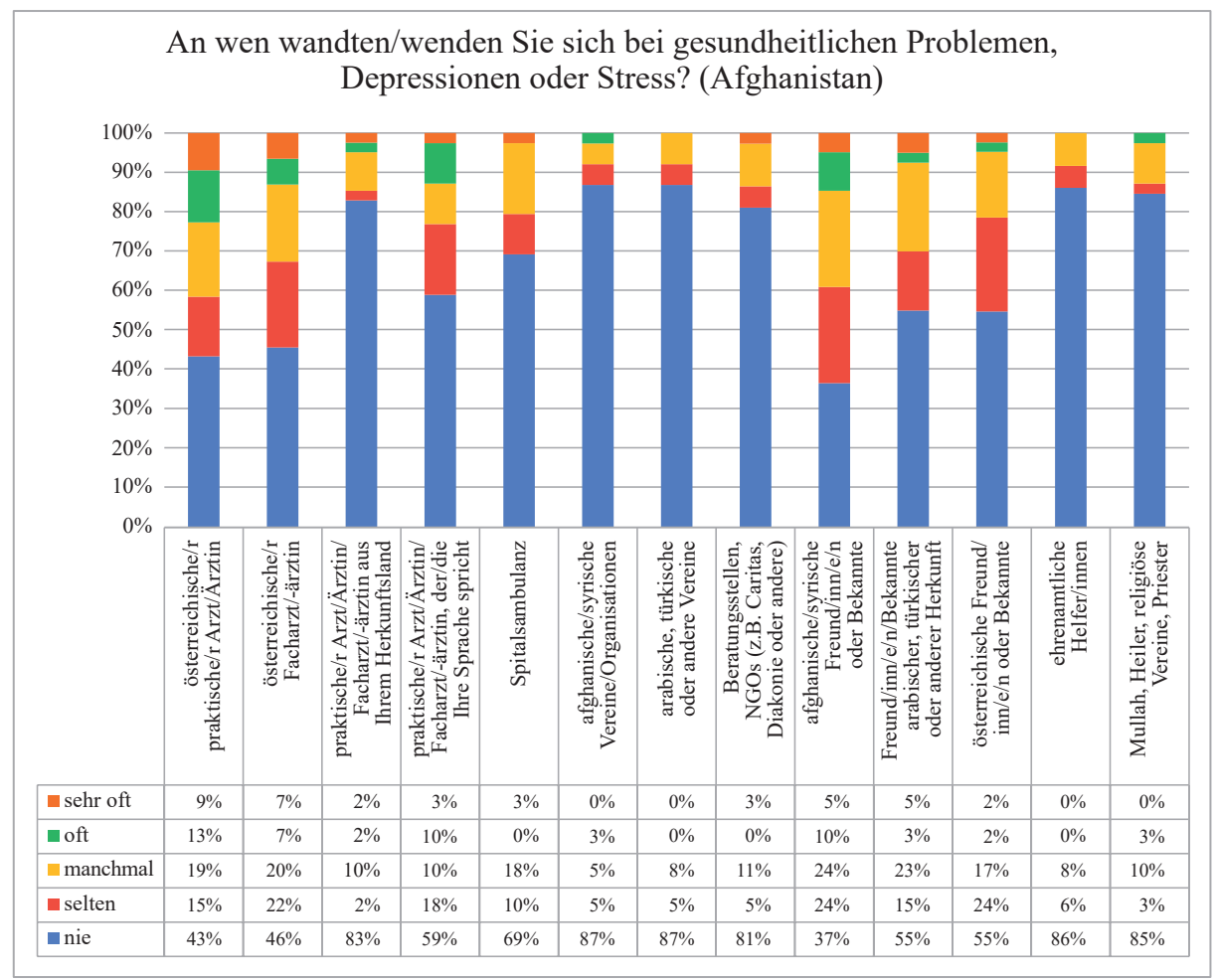

Quelle: eigene Erhebung 2020.

Was die afghanischen Befragten anbelangt (vgl. Abb. 4.5.1), so haben diese am wenigsten afghanische Organisationen sowie auch migrantische Vereine kontaktiert (jeweils $87 \%$ nie). Weitere $86 \%$ haben niemals Ehrenamtliche um Hilfe gebeten 
und $85 \%$ keine Mullahs oder religiösen Funktionäre, $81 \%$ haben keine NGOs oder Beratungsstellen und $83 \%$ (in weitgehender Ermangelung solcher) auch keine/n aus dem jeweiligen Herkunftsland stammende/n praktische/n Arzt/Ärztin kontaktiert.

Mit Abstand am öftesten haben sich Befragte aus Afghanistan an Freund/inn/e/n oder Bekannte aus der eigenen Community um Hilfe gewandt. 15\% taten dies oft bzw. sehr oft und 24\% manchmal. Eine wichtige Rolle spielten des Weiteren österreichische Allgemeinmediziner/innen, die von $22 \%$ oft bzw. sehr oft konsultiert wurden, von 19\% manchmal. Auch Fachärzt/ärztinn/e/n österreichischer Herkunft wurden um Hilfe gebeten, von $14 \%$ oft/sehr oft und von $20 \%$ manchmal. Etwas weniger häufig wandten sich Afghan/inn/en an Allgemeinmediziner/innen oder Fachärzt/inn/e/n mit muttersprachlicher Kompetenz, 13\% häufig und 10\% manchmal. Nahezu ebenso häufig wandten sich die Befragten an Freund/inn/e/n mit Migrationshintergrund (8\% häufig/ sehr häufig und 23\% manchmal) sowie des Weiteren an österreichische Freund/inn/e/n/

Abb. 4.5.2: An wen wandten sich die Befragten bei gesundheitlichen Problemen, Depression oder Stress? (Herkunft Syrien)

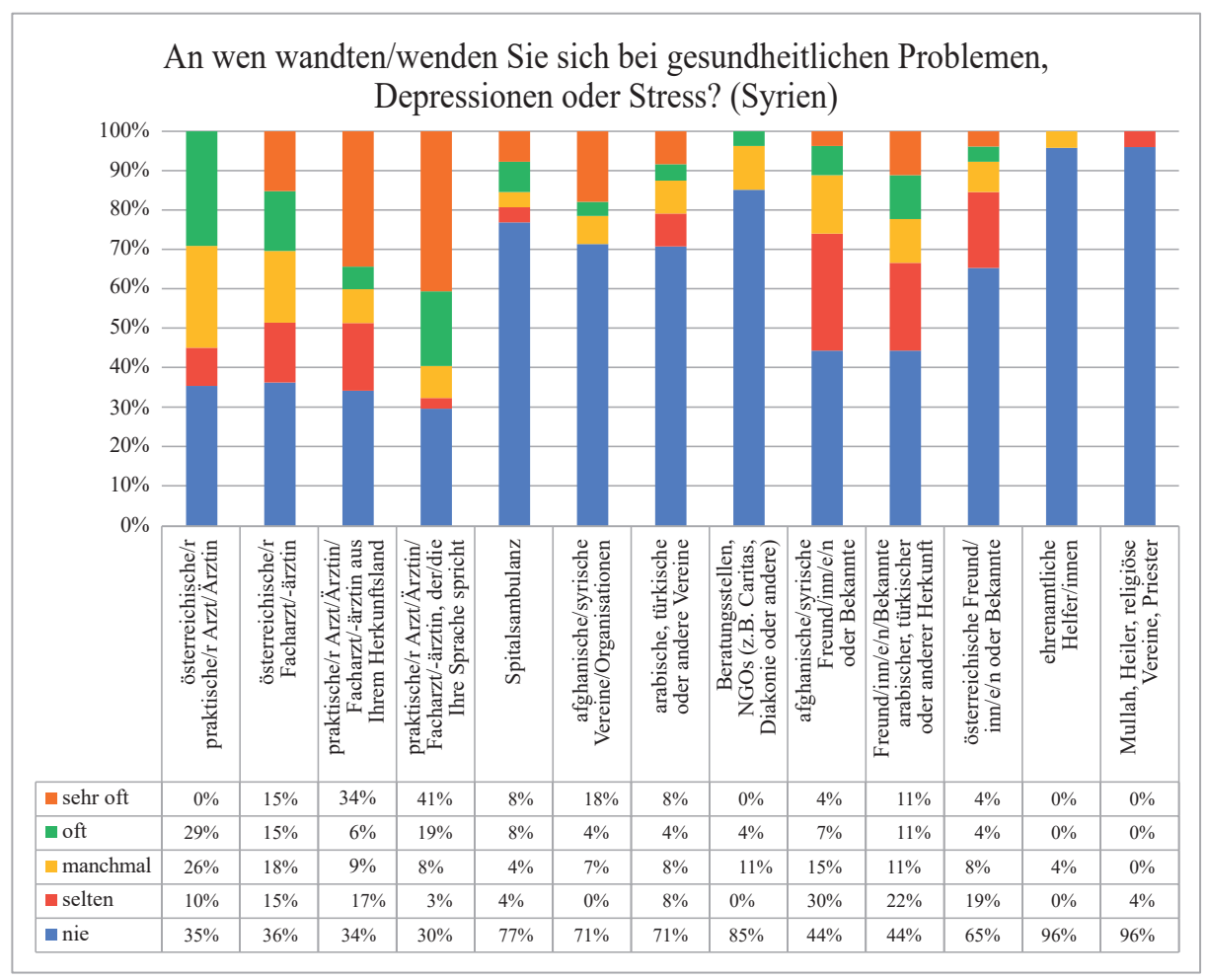

Quelle: eigene Erhebung 2020. 
Bekannte (4\% häufig/sehr häufig und 17\% manchmal). Spitalsambulanzen wurden deutlich weniger häufig aufgesucht als niedergelassene Ärzt/inn/e/n, was an einem aus dem Herkunftsland mitgebrachten Misstrauen gegenüber der Behandlung in Spitälern liegt, da das System der niedergelassenen Allgemeinmediziner/innen im Iran und Afghanistan unbekannt ist.

Auch im Fall der Syrer/innen gilt (vgl. Abb. 4.5.2), dass nahezu die Hälfte aller Befragten das größte Vertrauen in (Fach)ärzt/inn/e/n mit muttersprachlichen Kompetenzen legte. Im Vergleich dazu ist die Nennung von (Fach-)ärzt/inn/en aus dem Herkunftsland niedriger und die Nennung von österreichischen praktischen Ärzt/inn/en (mit nahezu einem Drittel der Befragten mit hoher Nennzahl) wie auch von Fachärzt/ inn/en bemerkenswert. Das Angebot an Ärzt/inn/en mit muttersprachlichen Kompetenzen in Arabisch ist in Wien im niedergelassenen wie auch im ambulanten Bereich höher als jenes mit Dari-Kenntnissen für Geflüchtete aus Afghanistan. Allerdings gibt es eine Reihe von praktischen und Fachärzt/inn/e/n die Farsi sprechen bzw. aus dem Iran stammen. Generell zeigen diese Nennungen ein vorhandenes Vertrauen in die österreichische medizinische Versorgung, wobei die Zugangs- bzw. Nutzungshürden vor allem sprachlicher Natur scheinen.

Einige Befragte aus Afghanistan teilten in den narrativen Interviews ihre Erfahrungen und Einschätzungen des afghanischen Gesundheitssystems mit. Diese Berichte waren gerade im Zusammenhang mit der COVID-19-Pandemie haarsträubend. Es ist nicht verwunderlich, wenn Menschen, die noch nicht so lange in Österreich sind und wenig Informationen über das Gesundheitssystem haben, noch kein Vertrauen aufbauen konnten. Jedoch dürfte sich das Vertrauen in selbiges relativ rasch entwickeln, dazu tragen natürlich Informationen über medizinische Belange in den jeweiligen Muttersprachen bei. Die Möglichkeit, sich von einem muttersprachlich versierten Arzt/Ärztin behandeln zu lassen, wurde sehr positiv aufgenommen und bestärkte das Vertrauen in das österreichische Gesundheitssystem. Auch die Vereine mit ihrer Informations- und Aufklärungstätigkeit trugen das Ihrige dazu bei.

Nahezu ein Fünftel aller Befragten meinte auch, dass die Vereine und Organisationen der Communities eine Anlaufstelle bei psychischen oder gesundheitlichen Problemen sind - ein ähnlich positiver Wert wurde Freund/inn/en oder Bekannten aus den jeweiligen Communities zuerkannt. Auffallend ist auch, dass weder Beratungsstellen von NGOs noch Freund/inn/e/n/Bekannte oder religiöse Einrichtungen/Funktionäre als Ansprechpartner bei physischen oder psychischen Erkrankungen gelten, sondern dass das Vertrauen in Fachpersonal sehr hoch ist und dieses direkt kontaktiert wird.

Es gab auch eine Reihe von Vereinsinitiativen oder losen Netzwerken, in denen sich medizinisches Personal mit Arabischkenntnissen zusammengeschlossen hat und neben telemedizinischer Erstberatung auch Informationskanäle eröffnet wurden, um die Patient/inn/en an medizinische Einrichtungen zu vermitteln. Eine Herausforderung dabei war, dass es am Anfang des ersten Lockdowns zu wenig Informationen darüber gab, welche Spezialambulanzen und Einrichtungen überhaupt geöffnet hatten. 
Die Themen und Aufgabenstellungen gingen oft weit über die medizinische Beratung hinaus und betrafen auch rechtliche, finanzielle und alltagsrelevante Hürden.

Eine Ärztin sprach auch ein Problem bezüglich der Informationsvermittlung in Sozialen Medien an. Waren am Anfang des Lockdowns die Informationen spärlich, wurden zunehmend durch eine Vielzahl von Gruppen unterschiedliche gesundheitsrelevante Informationen in den sozialen Medien geteilt, die allerdings auch manchmal nicht von Expert/inn/en verfasst wurden. Gerade in Bezug auf das diversifizierte und mehrschichtige Gesundheitssystem, das auch ohne eine Pandemie nicht leicht zu verstehen ist, wirkten sich Falschinformationen mehrfach negativ aus. Eine Ärztin verwies auch auf die Herausforderungen für NGOs oder Vereine, die ihre Expertise online angeboten hatten. Anfangs war es auch für ehrenamtlich tätige Mediziner/innen nicht einfach, adäquate Informationen über geöffnete medizinische Einrichtungen zu sammeln und den Klient/inn/en zur Verfügung zu stellen. Herausfordernd für Mediziner/ innen in diesen Netzwerken war auch die Beratung und Behandlung von Risikopatient/ inn/en, da sie oft über die gesamte Familiensituation besser Bescheid wussten und die Notwendigkeiten für soziale und rechtliche Unterstützung ebenso sahen.

\subsection{Genderspezifische Unterschiede hinsichtlich der Ansprech- personen und -institutionen bei gesundheitlichen Problemen}

Eine Reihe von geschlechtsspezifischen Unterschieden zeigten sich hinsichtlich der im Falle von gesundheitlichen Problemen kontaktierten Personen bzw. Institutionen (vgl. Tab. 7). So wurden österreichische Allgemeinmediziner/innen von mehr als einem Viertel der befragten Frauen, aber nur von 13,3\% der Männer oft aufgesucht. Konträr dazu war die Bereitschaft zum Aufsuchen eines/r österreichischen Facharztes/-ärztin bei den Männern erheblich höher (zu 24\% haben sie diese/n oft konsultiert, Frauen nur zu 13,8\%), 50\% der Frauen haben sogar nie ein/e/n österreichischen Facharzt/-ärztin kontaktiert. Ärzte aus dem Herkunftsland erfreuten sich bei den männlichen Befragten höheren Zuspruchs, da 24,4\% der Befragten diese sehr oft kontaktiert hatten (Frauen $8,6 \%, 74 \%$ hatten sogar nie ein/e/n Facharzt/-ärztin aus dem eigenen Herkunftsland konsultiert). Ähnlich fällt der Unterschied bei den Fachärzt/inn/en/innen aus, welche Farsi bzw. Arabisch sprechen. Spitalsambulanzen wurden wesentlich öfter von Männern aufgesucht (von 14,3\% manchmal), von 20\% der Frauen selten. An Vereine der eigenen Communities haben Männer sich bei gesundheitlichen Problemen oder Depressionen öfter gewandt (14,7\% oft bzw. sehr oft), während 15,6\% der Frauen eher Freund/inn/e/n /Bekannte aus den eigenen Communities kontaktiert haben (Männer nur zu 10\%). Österreichische Freund/inn/e/n bzw. Bekannte waren vor allem für die befragten Männer wichtig und wurden von 27\% manchmal kontaktiert. Mullahs/Heiler/religiöse Funktionäre wurden eher von Frauen kontaktiert, nämlich von 6,5\% der weiblichen Befragten oft oder zumindest manchmal, aber nur von rund 3\% der Männer manchmal und von 12\% selten. Generell rangierte die Kontaktaufnahme mit religiösen Funktionären an letzter Stelle. 
Tab. 7: An wen wandten sich männliche bzw. weibliche Befragte bei gesundheitlichen Problemen, Depression oder Stress? (gesamt)

\begin{tabular}{|c|c|c|c|}
\hline & männlich & weiblich & Gesamtergebnis \\
\hline \multicolumn{4}{|c|}{ österreichische/r praktische/r Arzt/Ärztin } \\
\hline manchmal & $11,11 \%$ & $15,38 \%$ & $13,10 \%$ \\
\hline nie & $40,00 \%$ & $41,03 \%$ & $40,48 \%$ \\
\hline oft & $13,33 \%$ & $25,64 \%$ & $19,05 \%$ \\
\hline sehr oft & $6,67 \%$ & $5,13 \%$ & $5,95 \%$ \\
\hline selten & $28,89 \%$ & $12,82 \%$ & $21,43 \%$ \\
\hline Gesamtergebnis & $100,00 \%$ & $100,00 \%$ & $100,00 \%$ \\
\hline \multicolumn{4}{|c|}{ österrreichische/r Facharzt/-ärztin } \\
\hline manchmal & $16,28 \%$ & $22,22 \%$ & $18,99 \%$ \\
\hline nie & $34,88 \%$ & $50,00 \%$ & $41,77 \%$ \\
\hline oft & $13,95 \%$ & $5,56 \%$ & $10,13 \%$ \\
\hline sehr oft & $6,98 \%$ & $5,56 \%$ & $6,33 \%$ \\
\hline sehr oft & $0,00 \%$ & $8,33 \%$ & $3,80 \%$ \\
\hline selten & $27,91 \%$ & $8,33 \%$ & $18,99 \%$ \\
\hline Gesamtergebnis & $100,00 \%$ & $100,00 \%$ & $100,00 \%$ \\
\hline \multicolumn{4}{|c|}{ Arzt/Ärztin/Facharzt/-ärztin aus Ihrem Herkunftsland } \\
\hline manchmal & $7,32 \%$ & $11,43 \%$ & $9,21 \%$ \\
\hline nie & $48,78 \%$ & $74,29 \%$ & $60,53 \%$ \\
\hline oft & $4,88 \%$ & $2,86 \%$ & $3,95 \%$ \\
\hline sehr oft & $24,39 \%$ & $8,57 \%$ & $17,11 \%$ \\
\hline selten & $14,63 \%$ & $2,86 \%$ & $9,21 \%$ \\
\hline Gesamtergebnis & $100,00 \%$ & $100,00 \%$ & $100,00 \%$ \\
\hline \multicolumn{4}{|c|}{ Arzt/Ärztin/Facharzt/-ärztin, der/die Ihre Sprache spricht } \\
\hline manchmal & $9,76 \%$ & $11,43 \%$ & $10,53 \%$ \\
\hline nie & $39,02 \%$ & $51,43 \%$ & $44,74 \%$ \\
\hline oft & $14,63 \%$ & $14,29 \%$ & $14,47 \%$ \\
\hline sehr oft & $24,39 \%$ & $17,14 \%$ & $21,05 \%$ \\
\hline selten & $12,20 \%$ & $5,71 \%$ & $9,21 \%$ \\
\hline Gesamtergebnis & $100,00 \%$ & $100,00 \%$ & $100,00 \%$ \\
\hline
\end{tabular}




\begin{tabular}{|c|c|c|c|}
\hline & männlich & weiblich & Gesamtergebnis \\
\hline \multicolumn{4}{|l|}{ Spitalsambulanz } \\
\hline manchmal & $14,29 \%$ & $0,00 \%$ & $7,69 \%$ \\
\hline nie & $68,57 \%$ & $76,67 \%$ & $72,31 \%$ \\
\hline oft & $5,71 \%$ & $0,00 \%$ & $3,08 \%$ \\
\hline sehr oft & $5,71 \%$ & $3,33 \%$ & $4,62 \%$ \\
\hline selten & $5,71 \%$ & $20,00 \%$ & $12,31 \%$ \\
\hline Gesamtergebnis & $100,00 \%$ & $100,00 \%$ & $100,00 \%$ \\
\hline \multicolumn{4}{|c|}{ afghanische/syrische Vereine/Organisationen } \\
\hline manchmal & $5,88 \%$ & $0,00 \%$ & $3,03 \%$ \\
\hline nie & $70,59 \%$ & $90,63 \%$ & $80,30 \%$ \\
\hline oft & $5,88 \%$ & $0,00 \%$ & $3,03 \%$ \\
\hline sehr oft & $8,82 \%$ & $6,25 \%$ & $7,58 \%$ \\
\hline selten & $8,82 \%$ & $3,13 \%$ & $6,06 \%$ \\
\hline Gesamtergebnis & $100,00 \%$ & $100,00 \%$ & $100,00 \%$ \\
\hline \multicolumn{4}{|c|}{ arabische, türkische oder andere Vereine } \\
\hline manchmal & $12,50 \%$ & $0,00 \%$ & $6,45 \%$ \\
\hline nie & $65,63 \%$ & $96,67 \%$ & $80,65 \%$ \\
\hline oft & $3,13 \%$ & $0,00 \%$ & $1,61 \%$ \\
\hline sehr oft & $3,13 \%$ & $3,33 \%$ & $3,23 \%$ \\
\hline selten & $15,63 \%$ & $0,00 \%$ & $8,06 \%$ \\
\hline Gesamtergebnis & $100,00 \%$ & $100,00 \%$ & $100,00 \%$ \\
\hline \multicolumn{4}{|c|}{ Beratungsstellen, NGOS (z.B. Caritas, Diakonie oder andere) } \\
\hline manchmal & $6,06 \%$ & $0,00 \%$ & $3,13 \%$ \\
\hline nie & $69,70 \%$ & $96,77 \%$ & $82,81 \%$ \\
\hline oft & $3,03 \%$ & $0,00 \%$ & $1,56 \%$ \\
\hline sehr oft & $3,03 \%$ & $0,00 \%$ & $1,56 \%$ \\
\hline selten & $18,18 \%$ & $3,23 \%$ & $10,94 \%$ \\
\hline Gesamtergebnis & $100,00 \%$ & $100,00 \%$ & $100,00 \%$ \\
\hline
\end{tabular}




\begin{tabular}{|c|c|c|c|}
\hline & männlich & weiblich & Gesamtergebnis \\
\hline \multicolumn{4}{|c|}{ afghanische/syrische Freund/inn/e/n oder Bekannte } \\
\hline manchmal & $25,00 \%$ & $28,13 \%$ & $26,47 \%$ \\
\hline nie & $36,11 \%$ & $43,75 \%$ & $39,71 \%$ \\
\hline oft & $8,33 \%$ & $9,38 \%$ & $8,82 \%$ \\
\hline sehr oft & $2,78 \%$ & $6,25 \%$ & $4,41 \%$ \\
\hline selten & $27,78 \%$ & $12,50 \%$ & $20,59 \%$ \\
\hline Gesamtergebnis & $100,00 \%$ & $100,00 \%$ & $100,00 \%$ \\
\hline \multicolumn{4}{|c|}{ Freund/inn/e/n/Bekannte arabischer, türkischer oder anderer Herkunft } \\
\hline manchmal & $18,92 \%$ & $16,67 \%$ & $17,91 \%$ \\
\hline nie & $40,54 \%$ & $63,33 \%$ & $50,75 \%$ \\
\hline oft & $8,11 \%$ & $3,33 \%$ & $5,97 \%$ \\
\hline sehr oft & $5,41 \%$ & $10,00 \%$ & $7,46 \%$ \\
\hline selten & $27,03 \%$ & $6,67 \%$ & $17,91 \%$ \\
\hline Gesamtergebnis & $100,00 \%$ & $100,00 \%$ & $100,00 \%$ \\
\hline \multicolumn{4}{|c|}{ österreichische Freund/inn/e/n oder Bekannte } \\
\hline manchmal & $27,03 \%$ & $16,13 \%$ & $22,06 \%$ \\
\hline nie & $48,65 \%$ & $70,97 \%$ & $58,82 \%$ \\
\hline oft & $5,41 \%$ & $0,00 \%$ & $2,94 \%$ \\
\hline sehr oft & $0,00 \%$ & $6,45 \%$ & $2,94 \%$ \\
\hline selten & $18,92 \%$ & $6,45 \%$ & $13,24 \%$ \\
\hline Gesamtergebnis & $100,00 \%$ & $100,00 \%$ & $100,00 \%$ \\
\hline \multicolumn{4}{|c|}{ ehrenamtliche Helfer/innen } \\
\hline manchmal & $6,67 \%$ & $0,00 \%$ & $3,33 \%$ \\
\hline nie & $80,00 \%$ & $100,00 \%$ & $90,00 \%$ \\
\hline selten & $13,33 \%$ & $0,00 \%$ & $6,67 \%$ \\
\hline Gesamtergebnis & $100,00 \%$ & $100,00 \%$ & $100,00 \%$ \\
\hline \multicolumn{4}{|c|}{ Mullah, Heiler, religiöse Vereine, Priester } \\
\hline manchmal & $3,03 \%$ & $3,23 \%$ & $3,13 \%$ \\
\hline nie & $84,85 \%$ & $93,55 \%$ & $89,06 \%$ \\
\hline oft & $0,00 \%$ & $3,23 \%$ & $1,56 \%$ \\
\hline selten & $12,12 \%$ & $0,00 \%$ & $6,25 \%$ \\
\hline Gesamtergebnis & $100,00 \%$ & $100,00 \%$ & $100,00 \%$ \\
\hline
\end{tabular}

Quelle: eigene Erhebung 2020. 


\section{Probleme infolge der Pandemie}

\subsection{Zugehörigkeit zu einer Risikogruppe}

Abb. 5.1: Zugehörigkeit zu einer Risikogruppe (gesamt und nach Herkunft)

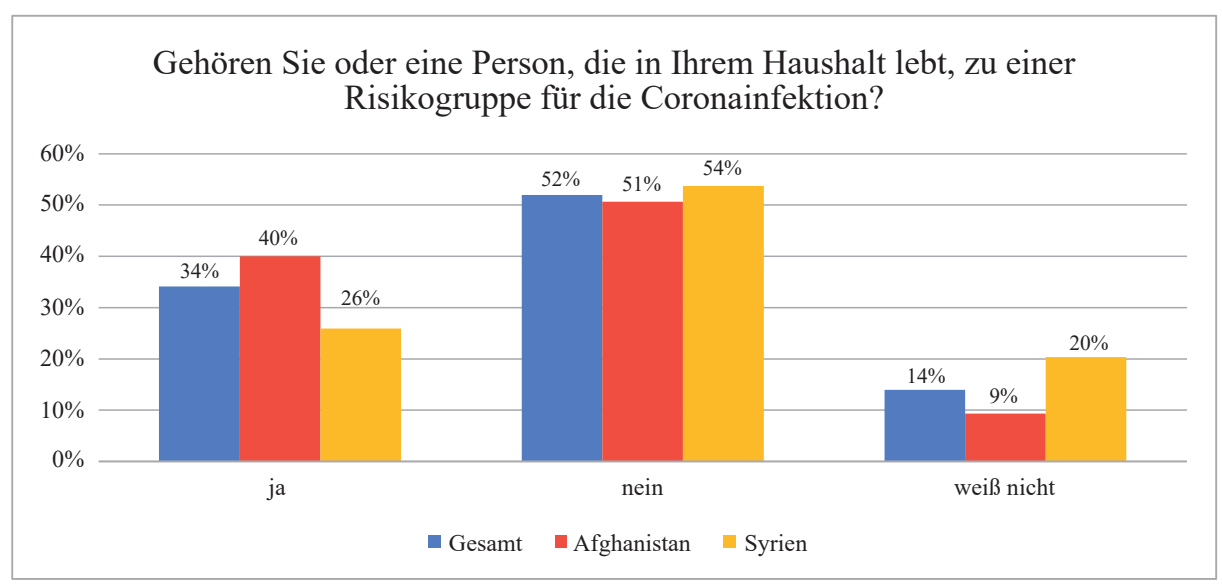

Quelle: eigene Erhebung 2020.

Zwar gehörte die Mehrzahl der Befragten (52\%) selbst nicht zu einer Risikogruppe (vgl. Abb. 5.1) und es lebten auch keine Angehörigen im selben Haushalt, die einer COVID-19-Risikogruppe zuzuordnen gewesen wären. Allerdings war der Anteil der Risikogruppen in den Haushalten der Afghan/inn/en erheblich höher (40\%) als bei den Syrer/inne/n (26\%). Auffällig ist hier der hohe Anteil an Personen, die mit ,weiß nicht“ antworteten. Im Schnitt des Samples 14\%, ein Fünftel in der Subgruppe aus Syrien, aber nur 9\% bei den Afghan/inn/en. Eventuell war in dieser ersten Phase der Pandemie das notwendige Wissen darüber, wer als Risikopatient/in galt, nicht ausreichend vorhanden. Das Wissen darüber hat sich ja auch in der Gesamtbevölkerung und durch zusätzliche medizinische Forschung erst entwickelt.

In der qualitativen Befragung berichtete eine Ärztin von einer Familie, bei der sich herausstellte, dass alle an COVID-19 erkrankt waren und der Vater eine intensivmedizinische Betreuung benötigte. Allerdings hatte die Familie Angst vor dem Verlust des Aufenthaltsstatus wegen der Nichteinhaltung des Interviewtermins. Aus Furcht vor rechtlichen Konsequenzen traute sich die Familie zuerst nicht die Rettung zu rufen. Erst durch die Intervention der arabischsprachigen Ärztin wurde der Vater hospitalisiert und musste intensivmedizinisch betreut werden. 


\subsection{Belastungen durch diverse Probleme}

Abb. 5.2 illustriert das unterschiedliche Belastungspotential infolge der zahlreichen Probleme, die sich während der Coronakrise für die Geflüchteten akkumulierten. Die Frage versuchte ein möglichst breites Spektrum an möglichen Problemstellungen abzudecken. Bezogen auf die Gesamtheit der Befragten stechen hier folgende Problembereiche hervor: Mit Abstand an der Spitze liegt Arbeitslosigkeit, dicht gefolgt von finanziellen und psychischen Problemen, weitere wichtige Problemfelder lagen im Homeschooling der Kinder, in sozialen Problemen und Isolation, Probleme mit der Einhaltung von Maßnahmen der Regierung sowie Unklarheit über die Situation der Familienmitglieder im Herkunftsland. So haben 28\% der Befragten angegeben, dass finanzielle Probleme stark bzw. völlig zutrafen, nur bei 16\% trafen diese gar nicht zu. Das Item Arbeitslosigkeit haben sogar 32\% als stark oder völlig zutreffend bewertet. Homeschooling wurde von $26 \%$ als starke Belastung bewertet, soziale Probleme und Isolation trafen auf $26 \%$ stark oder völlig zu und weitere $15 \%$ gaben an, dass diese teilweise auf ihre persönliche Lage zugetroffen hätte. Die Unklarheit über die Situation der Familie im Ausland traf auf 35\% völlig oder stark zu, auf weitere 12\% teilweise. Probleme mit Asylbehörden bzw. der Polizei oder die Ungewissheit über die Möglichkeit des Familiennachzugs waren nur selten genannte Items ebenso wie fehlende Kontakte $\mathrm{zu}$ afghanischen bzw. syrischen Vereinen oder gesundheitliche Probleme physischer Natur.

Nach Herkunftsländern betrachtet, zeigt Abb. 5.2.1, dass in den afghanischen Communities die folgenden Problemfelder infolge der Pandemie besonders virulent wurden: Homeschooling der Kinder (28\% völlig bzw. stark zutreffend), soziale Probleme/Isolation (27\% völlig bzw. stark zutreffend), finanzielle Probleme (26\% sowie weitere $17 \%$ auf die selbige teilweise zutrafen), psychische Probleme (26\%), Unklarheit über die Lage der Familie im Herkunftsland (26\%), Arbeitslosigkeit (19\% völlig bzw. stark zutreffend sowie $21 \%$, auf die dies teilweise zutraf), Unklarheit über den Familiennachzug sowie periphere Wohnstandorte mit je 13\%.

In der Kategorie geringster Belastungen wurden Probleme mit der Polizei (79\%), mit Asylbehörden und Vermieter/inn/en (jeweils 76\%), mit Nachbar/inne/n (72\%), physische Erkrankungen und Nachzug von Angehörigen (je 67\%) genannt, gefolgt von fehlenden Kontakten zu Vereinen der afghanischen Communities sowie zu anderen migrantischen Vereinen. Auch wurde Probleme, die aus Gewalt in der Familie resultierten, als gering eingestuft(64\%). Als zumindest teilweise zutreffend wurden Wohnprobleme (26\%), fehlende Kontakte zu Familienangehörigen (28\%), Probleme mit der Einhaltung der Regierungsmaßnahmen (17\%), fehlendes Internet (17\%), familiäre Probleme (17\%) sowie Probleme mit Nachbar/inne/n (16\%) klassifiziert. 
Abb. 5.2: Belastungen durch diverse Probleme in der Pandemie (gesamt)

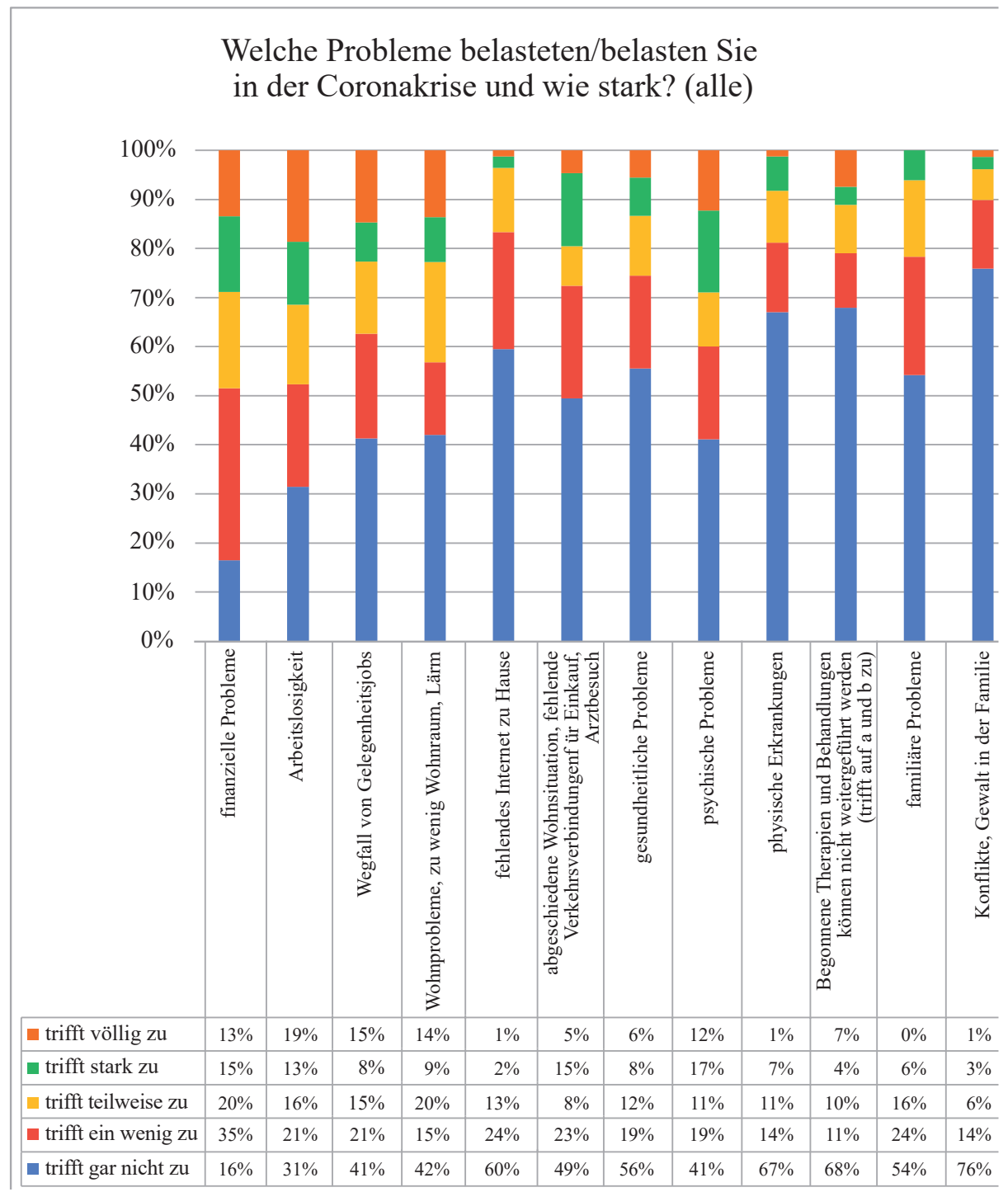

Quelle: eigene Erhebung 2020.

Die mehrfach belastende Problematik des Homeschoolings wurde auch in den Expert/inn/eninterviews deutlich. Für die NGOs war diese Aufgabe etwas völlig Neues und mit zahlreichen sogar täglich wechselnden Herausforderungen verbunden. Die Expert/inn/en klassifizierten das Homeschooling als eines der größten Problemfelder des 


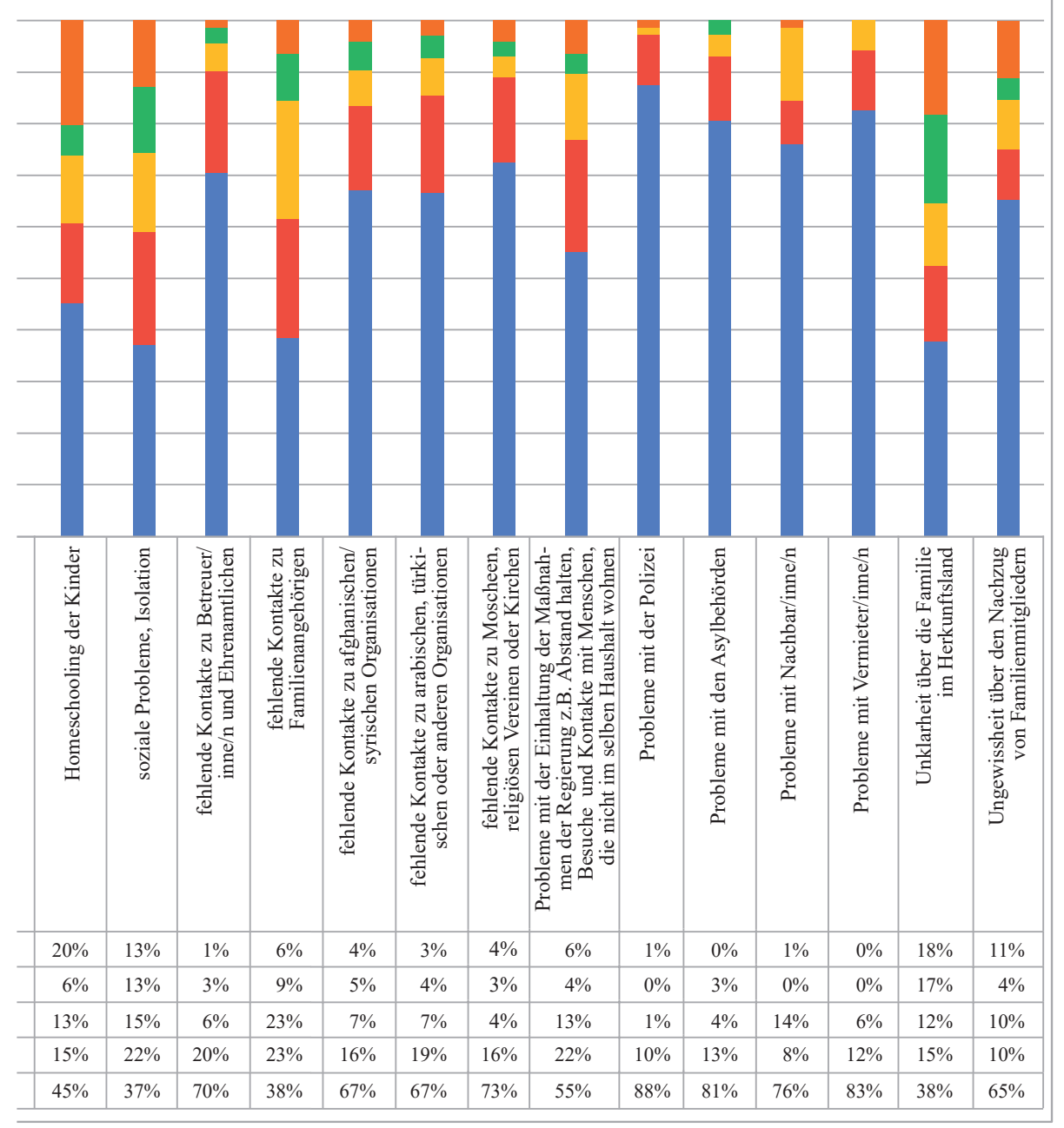

Lockdown, welches aufgrund der unterschiedlichen Ressourcen eine Schere zwischen Kindern aus ökonomisch bessergestellten, IT-mäßig gut ausgestatteten Haushalten und solchen mit mangelnder Ausstattung öffnete. Für die NGO-Mitarbeiter/innen sowie die Vereine resultierten daraus Herausforderungen, auf die rasch reagiert werden musste. 
Abb. 5.2.1: Belastungen durch diverse Probleme in der Pandemie (Herkunft Afghanistan)

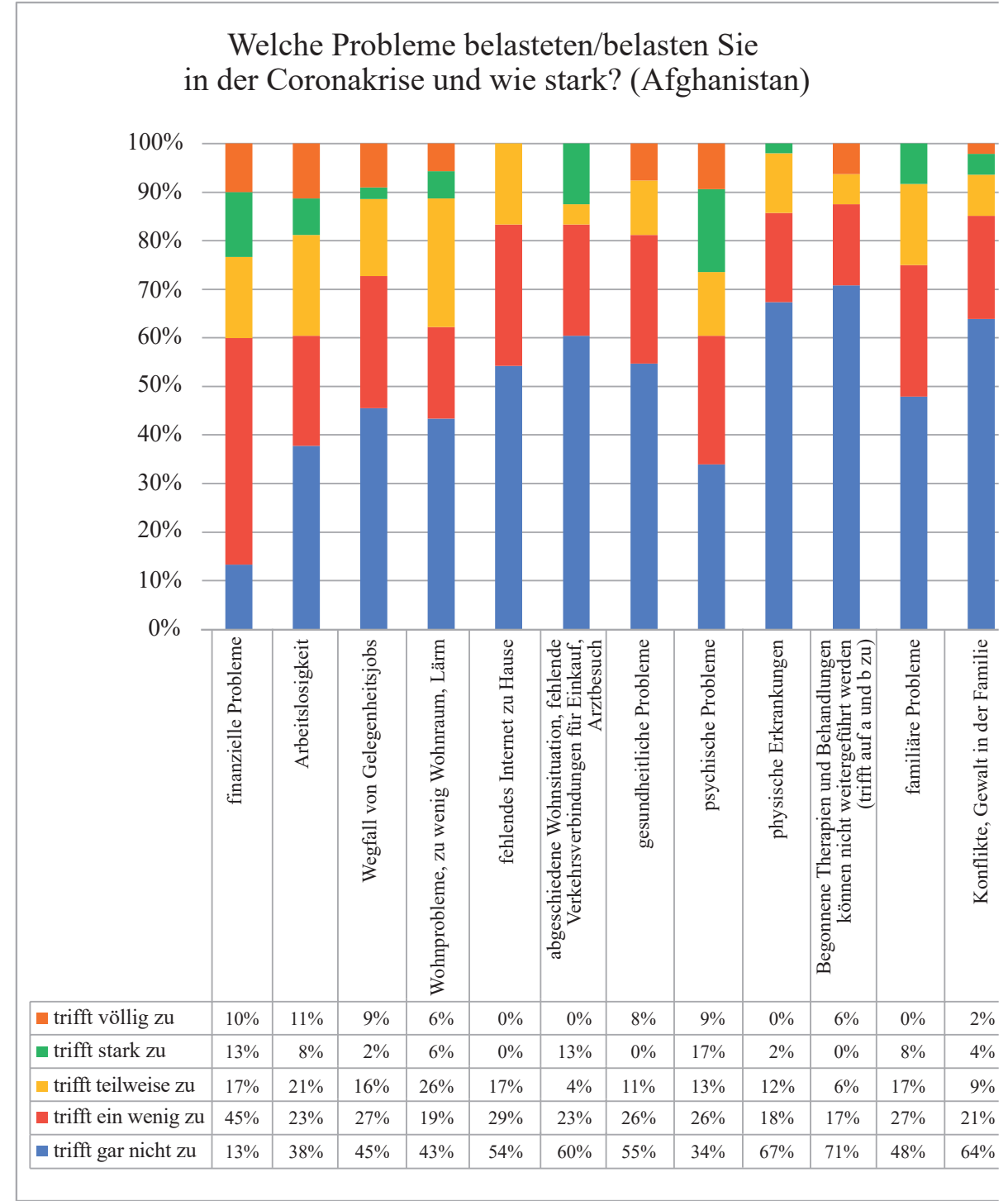

Quelle: eigene Erhebung 2020.

Eigentlich waren nur die wenigsten der Befragten vor dem Lockdown in den Bereich der Lernunterstützung involviert, obwohl einige NGOs solche Angebote, die sich auf direkten Kontakt bezogen, offerierten. Es mussten quasi über Nacht Online Hilfestel- 


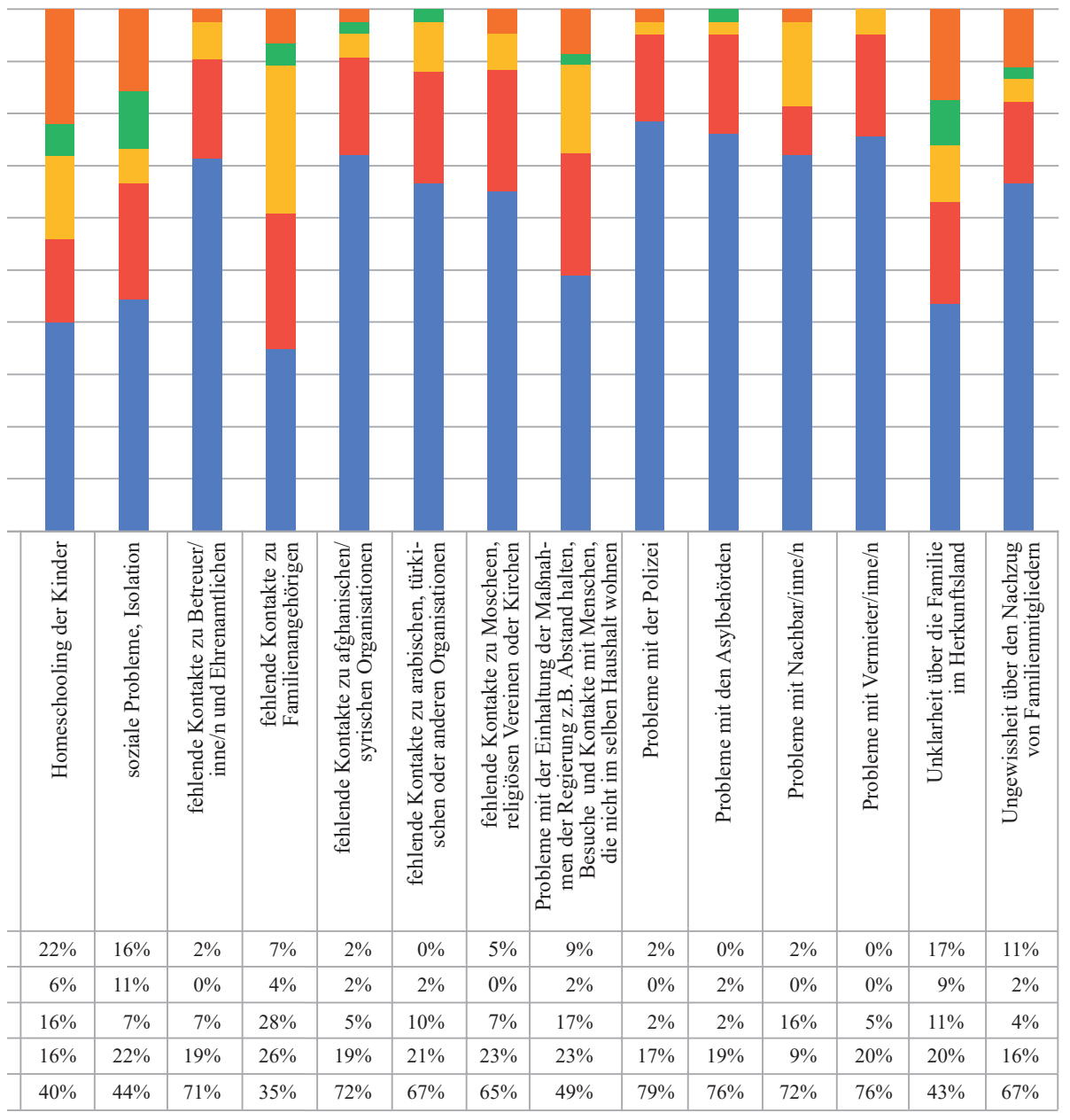

lungen im Zugang zu Lernmaterialien geschaffen werden, verschiedenartigste Defizite der digitalen Ausstattung in den Haushalten der Geflüchteten sowie hinsichtlich deren Umgang mit IT-Infrastrukturen kompensiert werden, den Eltern und Kindern Hilfe in der Bewältigung der Hausübungen zur Verfügung gestellt werden, sprachliche Defizite 
Abb. 5.2.2: Belastungen durch diverse Probleme in der Pandemie (Herkunft Syrien)

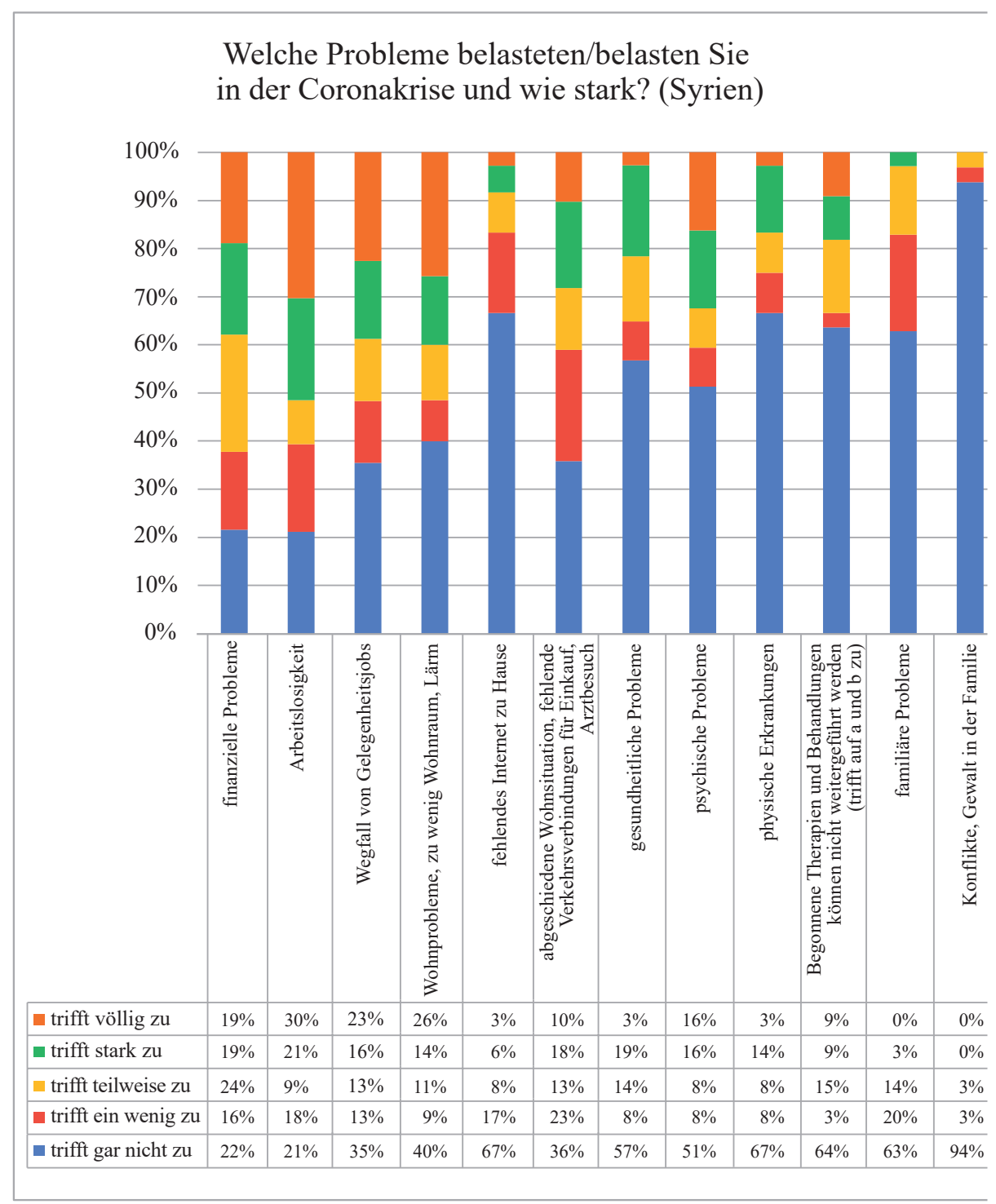

Quelle: eigene Erhebung 2020.

so gut als möglich ausgeglichen werden etc. Herausfordernd dabei war auch, dass man unterschiedliche Schultypen und -stufen (von der Volksschule über das Gymnasium bis hin zur Berufsschule) betreuen musste. Dafür waren einzelne Expert/inn/en nicht geschult oder vorbereitet. 


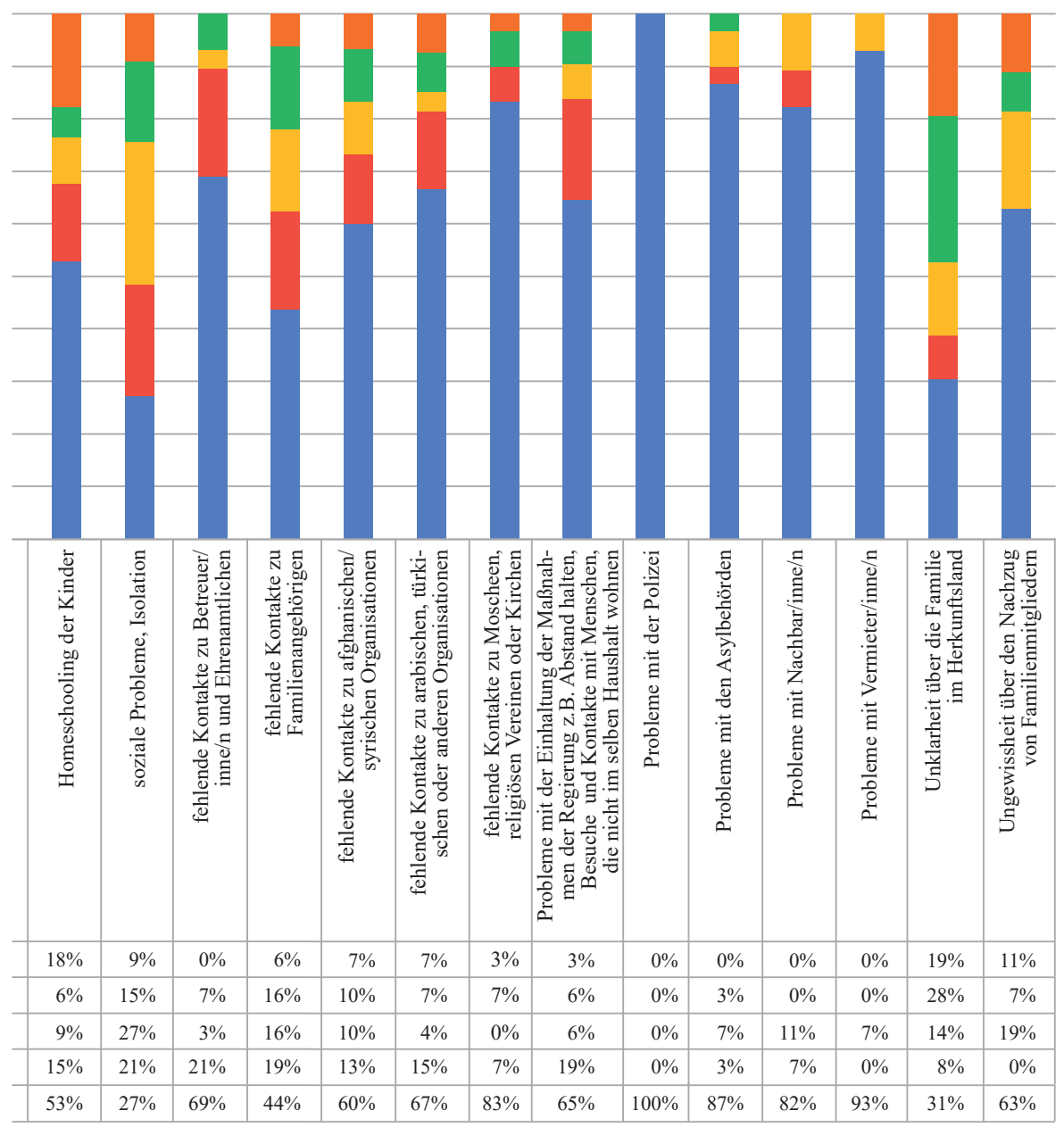

Die Expert/inn/en haben auch die generelle Problemsituation umrissen, d.h., wie schwierig das Homeschooling selbst für nichtgeflüchtete Familien zu bewältigen war und ist und verwiesen dabei auf die unterschiedlichen Ausgangssituationen der Kinder, wobei diese Diskrepanzen weiter verstärkt wurden. Dabei wurde insbesondere auch auf 
die zusätzlichen finanziellen Belastungen hingewiesen, wenn Lehrer/innen Unterlagen nur elektronisch zur Verfügung stellten und die Eltern für mehrere Kinder den Ausdruck dieser Materialien bestreiten mussten. Manche Vereine haben versucht, ehrenamtlich tätige Personen, (N)GOs und Initiativen mit ihren Klient/inn/en zu vernetzen, um auf diese Weise die Familien in Bildungsbelangen zu unterstützen.

Aus der Perspektive betroffener Schüler/innen und Eltern hat sich die Chancenungleichheit im Bildungssystem für die Kinder der Geflüchteten noch erheblich verschärft. $\mathrm{Zu}$ den ohnehin bestehenden sprachlichen Problemen, den unzureichenden Ressourcen, wie fehlende ruhige Arbeitsbereiche infolge knappen Wohnraums gesellten sich nun auch vielfältige technische Probleme, wie das Fehlen von Laptops und Druckern, langsame Internetanschlüsse, kaum Unterstützung durch die Eltern aufgrund von Bildungs- oder sprachlichen Mängeln oder Unkenntnissen der Lehr- und Lernsystems in Östereich. In der Phase nach dem Lockdown und relativer Normalität während des Sommers 2020 haben manche NGOs zum Zweck der Kompensation von Lerndefiziten zusätzliche Förderkurse für Kinder entwickelt oder mit sehr kreativen Initiativen (z.B. Vorlesen im Park) Defizite auszugleichen versucht. Die Klient/inn/en dieser Angebote waren jedoch durch die rasch wechselnden Vorschriften von Lockdowns und Öffnungen bereits sehr verunsichert und versuchten direkte Kontakte weiterhin auf ein Minimum zu reduzieren.

Die Kinder waren aber nicht nur im Hinblick auf das schulische Lernen besonders betroffen. Infolge der Sperre von Parks und anderer öffentlich zugänglicher Grünflächen mussten sie mit ihren Geschwistern wochenlang in Wohnungen verbringen, die meist nicht ausreichend Raum und Rückzugsmöglichkeiten boten und konnten ihren Bewegungs- und Erlebnisdrang in keiner Weise ausleben.

Auch familiäre Probleme wurden in vielerlei Hinsicht virulent, von einfachen Konflikten in der Partnerschaft bedingt durch finanzielle Probleme, beengte Wohnverhältnisse, die permanente häusliche Präsenz aller Familienmitglieder bis hin zu echten Gewaltkonstellationen. Hierbei wurden besonders zwei Problembereiche ausgemacht. Einerseits wirkten die Probleme und depressiven Stimmungen der Eltern auf die Kinder emotional ein, andererseits waren die Ehepartner/innen nun gezwungen mehr Zeit miteinander zu verbringen. Viele Problemkonstellationen waren komplex miteinander verzahnt. So konnte die Angst vor dem Alleinsein eine Flucht ins Zusammenwohnen bedingen, was jedoch aufgrund der erzwungenen mehrwöchigen Nähe und dem Mangel an Rückzugsmöglichkeiten erst recht zu Partnerschaftsproblemen führte. Probleme, die bereits vorher vorhanden waren, wurden dadurch verstärkt. Aus beiden Communities wurde vermehrt von Scheidungen berichtet, wobei die Initiativen meist von Frauen ausgehen. Manche Vereine haben versucht darauf zu reagieren, ein Verein hat spezifische Workshops zu Genderthemen angeboten.

Von den vielfältigen psychischen Problemen war soziale Isolation ein häufig genanntes und die Kontaktangebote, die die Vereine über Telefon oder soziale Medien angeboten haben, konnten dieses Defizit kaum kompensieren. 
Die Probleme waren jedoch nicht ausschließlich auf die Zeit des Lockdowns begrenzt, sondern haben sich danach fortgesetzt. In der Phase nach dem Lockdown gingen die Bemühungen der NGOs dahin, den erheblich ausgedünnten bzw. nicht selten sogar verlorene Kontakte zu ihren Klient/inn/en wiederherzustellen und somit wieder einigermaßen Normalität in ihren Beratungsleistungen anzubieten. Es galt dabei viele Belange aufzuholen und die wichtigsten Bereiche, wie rechtliche oder finanzielle Probleme, abzuhandeln. Dadurch hat sich für die Betreuer/innen die Beratungsintensität erheblich gesteigert.

Spezifische Probleme stellten sich auch bei jenen, die noch Asylwerber/innenstatus innehatten und somit noch in betreuten und oft überfüllten Asylquartieren lebten. Manche haben während des Lockdowns weitere Interviews oder andere rechtliche Erfordernisse im Asylverfahren erfüllen müssen. Für diese Menschen war die Unsicherheit mehrfach gegeben, sie steckten im Asylverfahren, wussten nicht wie ihre zukünftige Aufenthaltsmöglichkeit aussieht und generell nicht was in Zukunft mit ihnen passieren wird. Erschwerend kam hinzu, wenn in den Asylquartieren Coronafälle auftraten und eine Quarantäne vom Gesundheitsamt angeordnet wurde. Dies war für Betroffene und die Betreiberorganisationen kaum zu bewältigen - auch konnten Großquartiere nicht rasch aufgelöst werden. Betroffene erlebten auch Strafandrohungen, falls sie die Unterkunft verließen und nach draußen gingen. Expert/inn/en berichteten auch von den besonderen Herausforderungen bei einer Kumulation von Mehrfachbelastungen, wenn Bewohner/innen chronisch krank oder akut lebensbedrohlich erkrankt waren.

Aus dem sehr breiten Spektrum an Belastungen wurden bei den Befragten aus Syrien (vgl. Abb. 5.2.2) vorrangig folgende genannt: Arbeitslosigkeit, sei es allgemein oder der Verlust von Gelegenheitsjobs, die einen Zuverdienst bedeuteten, daraus resultierende finanzielle Probleme, gefolgt von Schwierigkeiten, die sich aus der Wohnsituation ergaben (z.B. Internetzugang). Mehr als zwei Drittel der Befragten nannten soziale Probleme, fehlende soziale Kontakte und Isolation, vor allem zu Betreuer/inne/n aus NGOs und Ehrenamtlichen; dies mehr noch als fehlende Kontakte zu syrischen Communities. Nur für ein Drittel war die Unklarheit über die Situation der Familienmitglieder im Herkunftsland nicht belastend, alle anderen Befragten gaben unterschiedliche Levels an Belastungen an. Für etwa 40\% der Befragten war die Unklarheit über den Familiennachzug belastend.

Zusammenfassend kann gesagt werden, dass sich bereits vor der Pandemie vorhandene problematische Situationen aufgrund der Wohnsituation und Isolation verstärkt haben. Einsamkeit und fehlende soziale Kontakte machten einigen der Befragten besonders zu schaffen. Die Beschaffung des technischen Equipments, um über das Internet Kontakte aufrechtzuerhalten, war auch nicht für alle möglich. Des Weiteren hatten vor allem ältere Menschen nicht die technischen Skills einen Computer zu bedienen oder mit Online- und Sozialen Medien zu operieren. Klient/inn/en eines Vereins regten daher Workshops und Schulungen an, um diese Skills zu vermitteln. Für manche war dies die Voraussetzung, um Kinder oder Enkelkinder im Homeschooling unterstützen oder auch um den Kontakt aufrechterhalten zu können. Als belastend 
stellte sich heraus, dass man die Maßnahmen zum Spracherwerb nicht weiterverfolgen konnte. Deutschkurse wurden abgesagt oder fanden nur mehr online statt. Und obwohl die Betroffenen mehr Zeit zur Verfügung hatten, hat dies den Spracherwerbsprozess verlangsamt, da die Menschen mehr auf sich allein gestellt waren.

Die Angst vor dem Verlust der Arbeit, die damit verbundenen finanziellen Probleme, der drohende Verlust der Wohnung war für manche, die es bereits geschafft hatten aus der Mindestsicherung herauszukommen, ein Rückschritt im Integrationsprozess. Manche konnten auf die Unterstützung der erweiterten Familie (Geschwister, Verwandte) zurückgreifen. Für andere bedeutete die Meldung beim AMS bzw. der Bezug der Mindestsicherung eine enorme Erschwerung ihrer Pläne, sobald als möglich um die Staatsbürgerschaft anzusuchen oder Familienmitglieder nachzuholen. Auch Kurzarbeit bedeutete für jene, die im Niedriglohnsektor tätig waren, einen enormen Einschnitt und Schwierigkeiten mit dem geringen Einkommen überhaupt den Lebensunterhalt zu finanzieren.

Ein weiterer Rückschlag im Integrationsprozess der Familie war für viele durch das Homeschooling bedingt. Für manche Eltern waren insbesondere die Herausforderungen für die Kinder, die erst eingeschult wurden, besonders hoch. Gerade das Bewusstsein, dass die ersten Schuljahre die wichtigsten für den gesamten Bildungsverlauf sind, haben die Zukunftsaussichten, die sie für ihre Kinder entwickelt haben, sehr beeinträchtigt und verstärkt Zukunftsängste hervorgerufen. Auch verfügten manche über einen Vergleich hinsichtlich der besseren Handhabung des Online-Unterrichts in anderen Schulen wie auch der generellen Handhabung in anderen Ländern. Von nahezu allen Befragten wurde erklärt, dass gerade das Homeschooling vor allem für die Frauen eine beträchtliche Mehrfachbelastung bedeutete. Eine Organisatorin eines Vereins erklärte, dass dieser auch mit unterschiedlichen Angeboten wie Diskussionen und Vorträgen bereits vor Corona auf die Mehrfachbelastungen der Frauen hingewiesen hatte und nach dem ersten Lockdown versuchte Angebote zu entwickeln, um dem gegenzusteuern (wie Nachmittagsbetreuung für die Kinder, zusätzliche künstlerische oder musikalische Beschäftigungen etc.). 


\subsection{Spezifische Probleme in Bezug auf die Erwerbs- und Ausbil- dungssituation}

Unter den in der Krise aufgetretenen Problemen waren vor allem finanzielle weit verbreitet. Stark zutreffend waren sie für $60 \%$ der vor der Coronakrise im Haushalt tätigen Befragten, sie trafen aber auch auf 50\% der Pensionist/inn/en und auf in Ausbildung stehende Personen zu sowie teilweise auf zwei Drittel der Arbeitsuchenden. Das Problem der Arbeitslosigkeit stellte sich in erster Linie für die vor der Pandemie in Ausbildung stehenden Befragten, Hausfrauen/-männer und Arbeitssuchende.

Die stärksten Ausprägungen zeigten finanzielle Probleme bei den zum Zeitpunkt der Erhebung in Ausbildung befindlichen Personen sowie Arbeitsunfähigen. Auch Arbeitslosigkeit betraf am stärksten Personen in Ausbildung, daneben aber auch Angestellte. Der Wegfall von Gelegenheitsjobs wurde von 66,7\% der zum Erhebungszeitpunkt im Haushalt tätigen Personen hervorgehoben, des Weiteren von jeweils der Hälfte der Praktikant/inn/en sowie der in Ausbildung stehenden Respondent/inn/en und auch von Selbständigen.

\subsection{Probleme in Bezug auf die Wohnsituation und Haushalts- struktur}

Spezifische Wohnprobleme wie zu wenig verfügbarer Wohnraum, kaum Rückzugsmöglichkeiten etc., welche in den qualitativen Interviews genannt worden waren, betrafen ein Drittel der Befragten in Wohnheimen und ein Fünftel der in Familienhaushalten wohnenden Respondent/inn/en, aber weniger die Alleinlebenden. Als teilweise zutreffend klassifizierten dies aber auch $48 \%$ der bei den Eltern lebenden Personen (vgl. Tab. 8). Insgesamt spielten Wohnprobleme aber eine eher untergeordnete Rolle.

Tab. 8: Wohnprobleme (gesamt)

\begin{tabular}{|l|r|r|r|r|r|r|}
\hline $\begin{array}{c}\text { Wohnprobleme, } \\
\text { zu wenig Wohn- } \\
\text { raum, Lärm }\end{array}$ & alleine & $\begin{array}{c}\text { bei den } \\
\text { Eltern }\end{array}$ & $\begin{array}{c}\text { bei der } \\
\text { eigenen } \\
\text { Familie }\end{array}$ & $\begin{array}{c}\text { Wohnge- } \\
\text { meinschaft }\end{array}$ & Wohnheim & $\begin{array}{c}\text { Gesamt- } \\
\text { ergebnis }\end{array}$ \\
\hline trifft ein wenig zu & $23,53 \%$ & $8,00 \%$ & $13,33 \%$ & $15,38 \%$ & $33,33 \%$ & $14,77 \%$ \\
\hline trifft gar nicht zu & $35,29 \%$ & $36,00 \%$ & $50,00 \%$ & $53,85 \%$ & $0,00 \%$ & $42,05 \%$ \\
\hline trifft stark zu & $5,88 \%$ & $4,00 \%$ & $13,33 \%$ & $15,38 \%$ & $0,00 \%$ & $9,09 \%$ \\
\hline trifft teilweise zu & $23,53 \%$ & $48,00 \%$ & $3,33 \%$ & $0,00 \%$ & $33,33 \%$ & $20,45 \%$ \\
\hline trifft völlig zu & $11,76 \%$ & $4,00 \%$ & $20,00 \%$ & $15,38 \%$ & $33,33 \%$ & $13,64 \%$ \\
\hline Gesamtergebnis & $\mathbf{1 0 0 , 0 0 \%}$ & $\mathbf{1 0 0 , 0 0 \%}$ & $\mathbf{1 0 0 , 0 0 \%}$ & $\mathbf{1 0 0 , 0 0 \%}$ & $\mathbf{1 0 0 , 0 0 \%}$ & $\mathbf{1 0 0 , 0 0 \%}$ \\
\hline
\end{tabular}

Quelle: eigene Erhebung 2020. 


\subsection{Genderspezifische Belastungen}

Spezifische Problemkonfigurationen haben die Geschlechter in sehr unterschiedlichem Ausmaß betroffen (vgl. Tab. 9). Mehr als die Hälfte der Befragten war nicht mit familiären Problemen kontrontiert, etwa 40\% teilweise (bzw. ein wenig), bei 6,8\% der Männer und 5,1\% der Frauen traf dies stark zu. In der Kategorie „trifft teilweise zu“ war der Anteil der Frauen fast doppelt so hoch wie jener der Männer (20,5\% zu 11,3\%). Mehr als drei Viertel gaben an, dass für sie Konflikte oder Gewalt in der Familie nicht zutreffend waren. Kompiliert man die beiden Kategorien „trifft teilweise zu“ und „trifft ein wenig zu“, so waren es immerhin 21,4\% der Männer und 18,9\% der Frauen, in deren Familien es zu Konflikten oder Gewalterfahrungen gekommen ist, das bedeutet also in summa bei rund einem Fünftel.

Tab. 9: Familiäre Probleme nach Geschlecht (gesamt)

\begin{tabular}{|c|c|c|c|}
\hline & männlich & weiblich & Gesamtergebnis \\
\hline \multicolumn{4}{|c|}{ familiäre Probleme } \\
\hline trifft ein wenig zu & $25,00 \%$ & $23,08 \%$ & $24,10 \%$ \\
\hline trifft gar nicht zu & $56,82 \%$ & $51,28 \%$ & $54,22 \%$ \\
\hline trifft stark zu & $6,82 \%$ & $5,13 \%$ & $6,02 \%$ \\
\hline trifft teilweise zu & $11,36 \%$ & $20,51 \%$ & $15,66 \%$ \\
\hline Gesamtergebnis & $100,00 \%$ & $100,00 \%$ & $100,00 \%$ \\
\hline \multicolumn{4}{|c|}{ Konflikte, Gewalt in der Familie } \\
\hline trifft ein wenig zu & $11,90 \%$ & $16,22 \%$ & $13,92 \%$ \\
\hline trifft gar nicht zu & $71,43 \%$ & $81,08 \%$ & $75,95 \%$ \\
\hline trifft stark zu & $4,76 \%$ & $0,00 \%$ & $2,53 \%$ \\
\hline trifft teilweise zu & $9,52 \%$ & $2,70 \%$ & $6,33 \%$ \\
\hline trifft völlig zu & $2,38 \%$ & $0,00 \%$ & $1,27 \%$ \\
\hline Gesamtergebnis & $100,00 \%$ & $100,00 \%$ & $100,00 \%$ \\
\hline \multicolumn{4}{|c|}{ Homeschooling der Kinder } \\
\hline trifft ein wenig zu & $21,43 \%$ & $9,52 \%$ & $15,48 \%$ \\
\hline trifft gar nicht zu & $52,38 \%$ & $38,10 \%$ & $45,24 \%$ \\
\hline trifft stark zu & $4,76 \%$ & $7,14 \%$ & $5,95 \%$ \\
\hline trifft teilweise zu & $4,76 \%$ & $21,43 \%$ & $13,10 \%$ \\
\hline trifft völlig zu & $16,67 \%$ & $23,81 \%$ & $20,24 \%$ \\
\hline Gesamtergebnis & $100,00 \%$ & $100,00 \%$ & $100,00 \%$ \\
\hline
\end{tabular}

Quelle: eigene Erhebung 2020. 
Neuerlich ist hervorzuheben, dass das Homeschooling eine der erheblichsten Herausforderung des Lockdowns war, sofern Kinder im Haushalt lebten. Völlig zutreffend haben hier 23,8\% der Frauen und 16,7\% der Männer angekreuzt, subsumiert man die Kategorien ,trifft ein wenig zu“ und „trifft teilweise zu“, so ergeben sich Werte von 26,2\% bei den Männern und 31\% bei den Frauen, denen das Homeschooling sichtlich Probleme bereitet hat. 


\section{Bedeutung der Religion in der Coronakrise}

Abb. 6.1: Die Bedeutung der Religion in der Coronakrise (gesamt und nach Herkunft)

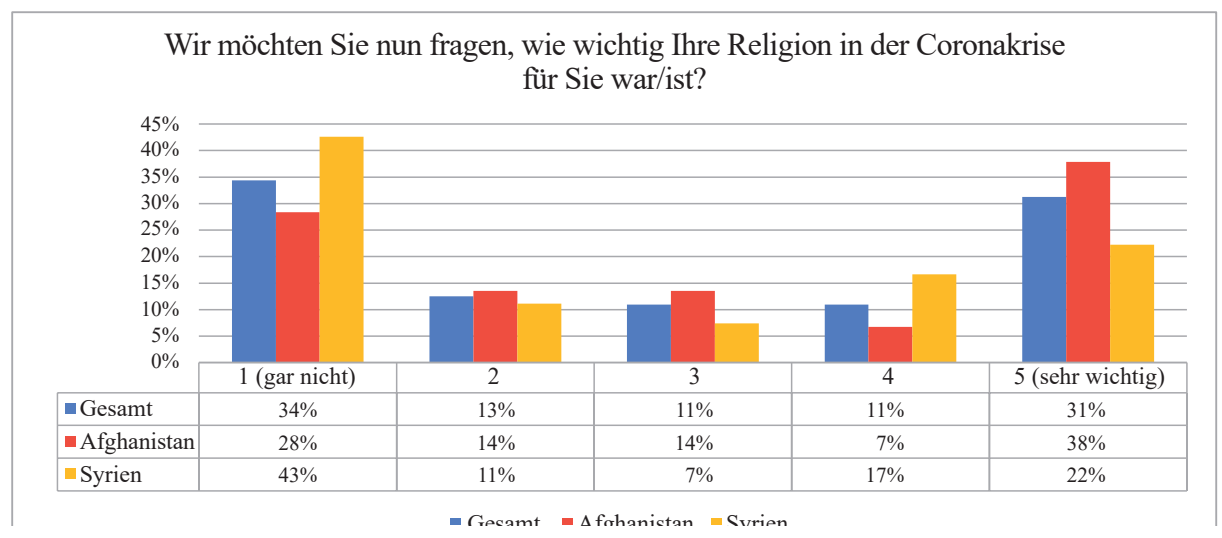

Quelle: eigene Erhebung 2020.

Um die Bandbreite an Ressourcen abzubilden, auf die geflüchtete Personen in der Krise zurückgreifen konnten, wurde auch die Relevanz von Religion, von religiösen Einrichtungen und deren Angeboten erhoben. Religion kann im Kontext von Krankheit und Leiden allgemein, also auch in Zeiten der Pandemie, eine sehr ambivalente Rolle spielen. Religiöse Menschen können durch Glaubensinhalte und -praxen vielleicht besser psychisch unbeschadet durch solche Ausnahmesituationen kommen und sich eventuell auch eher vor Infektionen geschützt fühlen. Unter Umständen kann dies auch zu einem riskanteren Verhalten beitragen. Religion kann aber auch eine Ressource sein, um Krankheiten besser durchzustehen und mit Depressionen und Ängsten besser umzugehen.

In der Befragung zeigte sich eine ausgeprägte Polarisierung zwischen jenen Befragten, für die Religion keine Bedeutung hatte, und jener ebenfalls sehr großen Gruppe, für die Religion eine sehr wichtige Rolle spielte (vgl. Abb. 6.1). So war für $34 \%$ Religion gar nicht und für 32\% sehr wichtig. Die mittleren Positionen in Bezug auf die Religiosität waren mit jeweils $11 \%$ bzw. 13\% deutlich schwächer besetzt.

Sehr deutlich manifestiert sich ein Unterschied zwischen den beiden Herkunftsgruppen. Gar nicht wichtig war Religion für 43\% der syrischen Respondent/inn/en, aber nur für 28\% der Afghan/inn/en. Als sehr wichtig hingegen bewerteten 38\% der Afghan/ inn/en und nur 22\% der Syrer/innen ihren Glauben. Ein sehr deutlicher Unterschied trat auch in der Kategorie ,wichtig“" zutage. Diese haben $17 \%$ der syrischen und nur 7\% der afghanischen Befragten angekreuzt, während in der mittleren Kategorie die Afghan/inn/ 
en mit 14\% dominierten (Syrer/innen 7\%). Diese Unterschiede hinsichtlich der relativen Bedeutung von Religion wurden auch in früheren Studien bereits erhoben. ${ }^{12}$

Besonders in der Anfangsphase der Coronapandemie glaubten manche Gläubige, dass sie sich durch ihren Glauben vor Infektionen schützen könnten. Auch wurden in manchen Communities wie in der österreichischen Gesellschaft auch Verschwörungstheorien verbreitet. Die Vereine der Communities haben hier wichtige Überzeugungsarbeit geleistet.

Die Moscheen in Österreich haben sich weitgehend an die Verordnungen der Regierung gehalten, blieben geschlossen und die Gläubigen wurden aufgefordert, das Gebet nach Hause zu verlegen. Auch hier haben die afghanischen Vereine entsprechende Informationen in die Muttersprachen übersetzt und die Verantwortlichen in den Moscheen sowie die Gläubigen über die aktuell zu befolgenden Maßnahmen instruiert sowie für deren Einhaltung plädiert. Vereinzelt wurden Videoaufnahmen erstellt, die dann von den Gläubigen im Internet abgerufen werden konnten.

Als der Besuch von religiösen Stätten nach dem Lockdown wieder möglich war, wurden in den Moscheen strenge Regeln erlassen. Gläubige mussten ihre eigenen Gebetsteppiche mitbringen, nur Männer hatten Zutritt, für Frauen blieben manche Moscheen geschlossen. Ein befragter Exprete, der auch eine Moschee betreut, berichtete dass vor der Pandemie an Samstagen und Sonntagen spezielle Programme für Frauen und Kinder angeboten worden waren. Man konnte einander treffen, gemeinsam Essen, Farsi lernen etc. Seit Beginn der Coronamaßnahmen waren diese Angebote ausgesetzt. Laut diesem Experten hat sich der Wegfall dieser Möglichkeiten sozialer Vernetzung mangels Alternativen für Frauen als problematisch erwiesen.

Vereinzelt wurde von religiösen Gemeinschaften berichtet, die sich nicht an die Verordnungen gehalten und Gebetstreffen organisiert haben. Diese strenggläubigen Gruppierungen stehen wissenschaftlichen Erkenntnissen distanziert gegenüber und orientieren sich am Koran und religiösen Gepflogenheiten.

Einzelne Religionsgemeinschaften bauten auch ihr Online-Angebot erheblich aus (s.u.).

Des Weiteren wurde nachgefragt, inwieweit Gebete zur Bewältigung und Reduktion von Ängsten und Stress bedeutsam waren. Analog zum Verteilungsbild hinsichtlich der Wichtigkeit von Religion allgemein tritt eine Polarisierung zwischen völliger Unwichtigkeit und sehr hoher Bedeutung zutage (vgl. Abb. 6.2). So war das Beten für 29\% aller Respondent/inn/en sehr wichtig und für 12\% immerhin wichtig, für $35 \%$ völlig unwichtig sowie für 14\% eher unwichtig.

Ähnlich der Bedeutung von Religion im Allgemeinen zeigen sich unterschiedliche Grade der Relevanz des Betens. So ist der Prozentsatz jener, für die das Gebet gar nicht oder eher nicht wichtig war, bei den Afghan/inn/en mit 43\% erheblich geringer als bei

$12 \quad$ Vgl. Bauer et al. 2017. 
den Syrer/inne/n mit 57\%. Eine sehr wichtige bzw. wichtige Rolle spielte das Beten hingegen für nahezu die Hälfte im Sample aus Afghanistan, aber nur für ein Drittel im Falle der Syrer/innen.

Abb. 6.2: Wichtigkeit des Betens zur Stressbewältigung (gesamt und nach Herkunft)

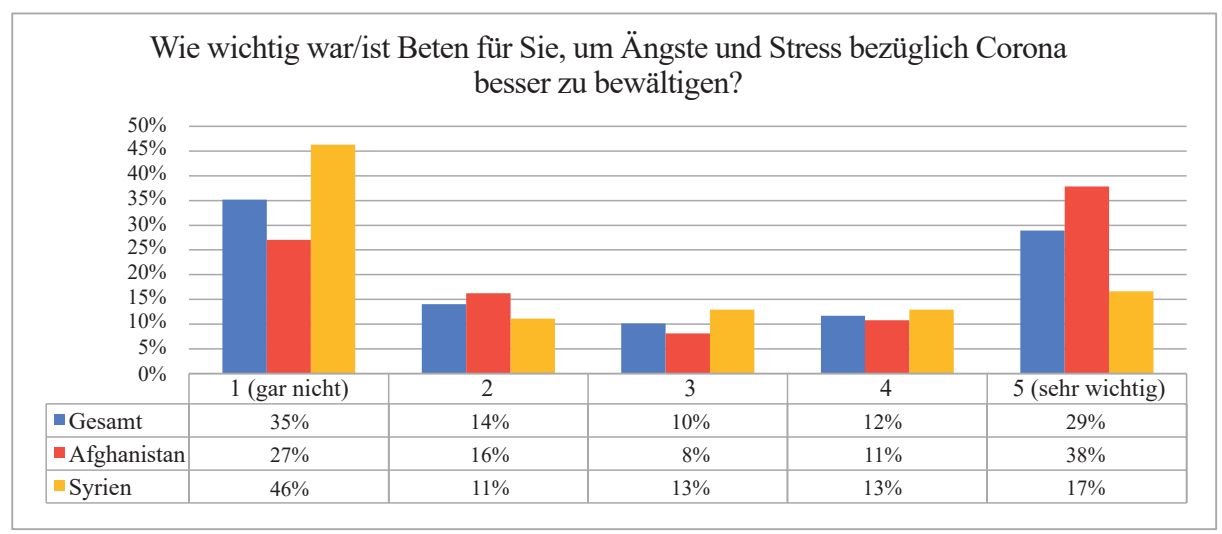

Quelle: eigene Erhebung 2020.

Das Beten spielte also für einen Teil der afghanischen Communities eine wichtige Rolle als Trost und Zuflucht, wobei die Wichtigkeit der Religion individuell höchst unterschiedlich bewertet wurde. Aus der Perspektive der Expert/inn/en der NGOs stellt sich die Situation ebenfalls uneinheitlich dar. Ein maßgeblicher Experte und Repräsentant einer Glaubensgemeinschaft meinte, keine generelle Beurteilung zur Rolle der Religion im Corona-Kontext abgeben zu können. Innerhalb der Gruppen der Geflüchteten aus Afghanistan und Syrien gibt es viele unterschiedliche Religionsgemeinschaften und es lässt sich kein genereller Trend feststellen. Für einige Afghan/inn/en stellte jedoch der Glaube zweifellos eine Stütze da. Eine Expertin stellte fest, dass religiöse Funktionäre als primäre Ansprechpersonen in der Coronakrise nicht besonders wichtig waren. Respondent/inn/en meinten, dass ihnen die Spiritualität und Alltagsrituale eher dafür wichtig waren, um die allgemeinen Belastungen zu meistern. Jedoch stellt für die Befragten Religion durchwegs keine Alternative zu medizinischen oder therapeutischen Behandlungen dar und auch befragte Vertreter/innen von Vereinen und Organisationen sahen die Informationsvermittlung über infektionsminimierende Maßnahmen und notwendige medizinische Betreuung als besonders wichtig an. 
Abb. 6.3: Inanspruchnahme von Online-Angeboten der Religionsgemeinschaften (gesamt und nach Herkunft)

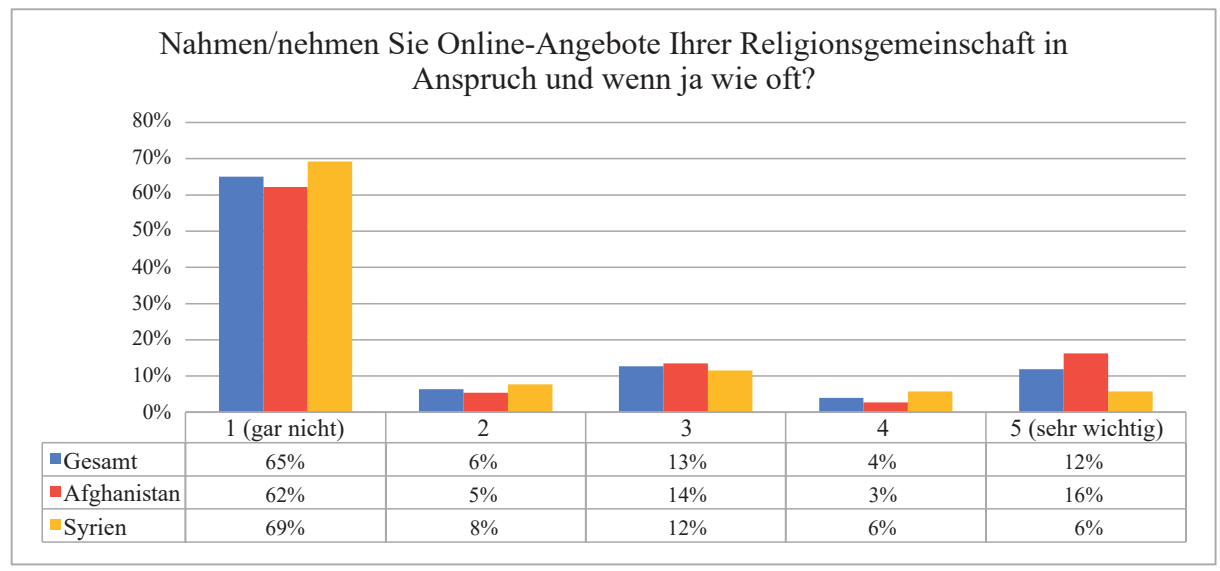

Quelle: eigene Erhebung 2020.

Aufgrund der ausgeprägten Bedeutung der Religion im Allgemeinen sowie des Gebets im Besonderen für immerhin ein rundes Drittel der Befragten war die Annahme naheliegend, dass auch Online-Angebote der Religionsgemeinschaften in Anspruch genommen worden sind (vgl. Abb. 6.3). Die Resultate der Befragung ließen diesen Schluss jedoch nicht zu. Nur 12\% aller Befragten nahmen solche Angebote als sehr wichtig in Anspruch, während 65\% diese überhaupt nicht genutzt hatten. 13\% meinten, an diesen Angeboten zumindest manchmal partizipiert zu haben. Die Relevanz von Online-Angeboten wurde seitens der Afghan/inn/en zu 16\% als sehr wichtig bewertet, während nur 6\% der syrischen Subgruppe diese Einschätzung abgegeben haben. Dies obwohl mit dem Lockdown eine stark steigende Wichtigkeit von Online-Angeboten auch seitens der Repräsentant/inn/en der NGOs betont wurde.

Vertreter/innen einer Hilfsorganisation für Syrer/innen unterschiedlicher christlicher Konfessionen erklärten, dass Sonntagsmessen online übertragen wurden. Priester haben auch mit Familien Katechesen oder Rosenkranzgebete organisiert, um sich mit Verwandten aus Syrien und jenen, die transnational verstreut leben, virtuell zum Gebet treffen zu können.

Die Relevanz der Religion als Hilfsmittel zur Bewältigung der Coronakrise war nicht, wie eigentlich anzunehmen, in der Gruppe ohne Bildungsabschlüsse am größten. In dieser gaben nämlich $50 \%$ an, dass Religion eine sehr geringe Bedeutung gehabt hätte. In den anderen Bildungskategorien rangierte die sehr geringe Bedeutung bei Werten zwischen 30,4\% (sekundäre Bildung), 34,4\% (primäre Abschlüsse) und 37,8\% (tertiäre Bildung). Sehr hohe Wichtigkeit kam dem Glauben vor allem in der Kategorie sekundärer Bildung (37\%) zu, weiters bei der Universitätsabsolvent/inn/en (29,7\%) und bei einem Viertel der Befragten mit primären Abschlüssen (vgl. Tab. 10). 
Tab. 10: Relevanz der Religion für Befragte mit unterschiedlichen Bildungsabschlüssen (gesamt)

\begin{tabular}{|l|r|r|r|r|r|}
\hline & \multicolumn{1}{|c|}{$\begin{array}{c}\text { kein } \\
\text { Bildungs- } \\
\text { abschluss }\end{array}$} & $\begin{array}{c}\text { primärer } \\
\text { Bildungs- } \\
\text { abschluss }\end{array}$ & $\begin{array}{c}\text { sekundärer } \\
\text { Bildungs- } \\
\text { abschluss }\end{array}$ & $\begin{array}{c}\text { tertiärer } \\
\text { Bildungs- } \\
\text { abschluss }\end{array}$ & \multicolumn{1}{c|}{$\begin{array}{c}\text { Gesamt- } \\
\text { ergebnis }\end{array}$} \\
\hline $\begin{array}{l}1 \text { (= sehr geringe } \\
\text { Bedeutung) }\end{array}$ & $50,00 \%$ & $34,38 \%$ & $30,43 \%$ & $37,84 \%$ & $34,38 \%$ \\
\hline 2 & $12,50 \%$ & $12,50 \%$ & $6,52 \%$ & $18,92 \%$ & $12,50 \%$ \\
\hline 3 & $25,00 \%$ & $18,75 \%$ & $8,70 \%$ & $5,41 \%$ & $10,94 \%$ \\
\hline 4 & $0,00 \%$ & $9,38 \%$ & $17,39 \%$ & $8,11 \%$ & $10,94 \%$ \\
\hline $\begin{array}{l}5(=\text { sehr hohe } \\
\text { Bedeutung) }\end{array}$ & $12,50 \%$ & $25,00 \%$ & $36,96 \%$ & $29,73 \%$ & $31,25 \%$ \\
\hline Gesamtergebnis & $\mathbf{1 0 0 , 0 0 \%}$ & $\mathbf{1 0 0 , 0 0 \%}$ & $\mathbf{1 0 0 , 0 0 \%}$ & $\mathbf{1 0 0 , 0 0 \%}$ & $\mathbf{1 0 0 , 0 0 \%}$ \\
\hline
\end{tabular}

Quelle: eigene Erhebung 2020. 


\section{UNTERSTÜTZUNGSANGEBOTE}

Angesichts der Komplexität und Vielzahl der durch die Pandemie hervorgerufenen Probleme wurde der Bedarf an Unterstützung in einzelnen Lebensbereichen erhoben (vgl. Abb. 7.1). Mit Abstand die meisten Befragten erklärten, dass sie im Kontext der Coronakrise emotionale Unterstützung (29\%) benötigten. Dann folgte die Hilfe bei der Kinderbetreuung und vor allem beim Homeschooling (17\%). Für die finanzielle Unterstützung von Freund/inn/en oder Verwandten haben 14\% zusätzliche Hilfe benötigt und ebenso 14\% brauchten Hilfe beim Einkaufen. Finanzielle Unterstützung für das eigene Geschäft war zu 10\% gefragt und 9\% gaben Übersetzungsbedarf an, um die Maßnahmen und Informationen der Regierung verstehen zu können.

Abb. 7.1: In welchen Bereichen wurde Unterstützung benötigt? (gesamt und nach Herkunft)

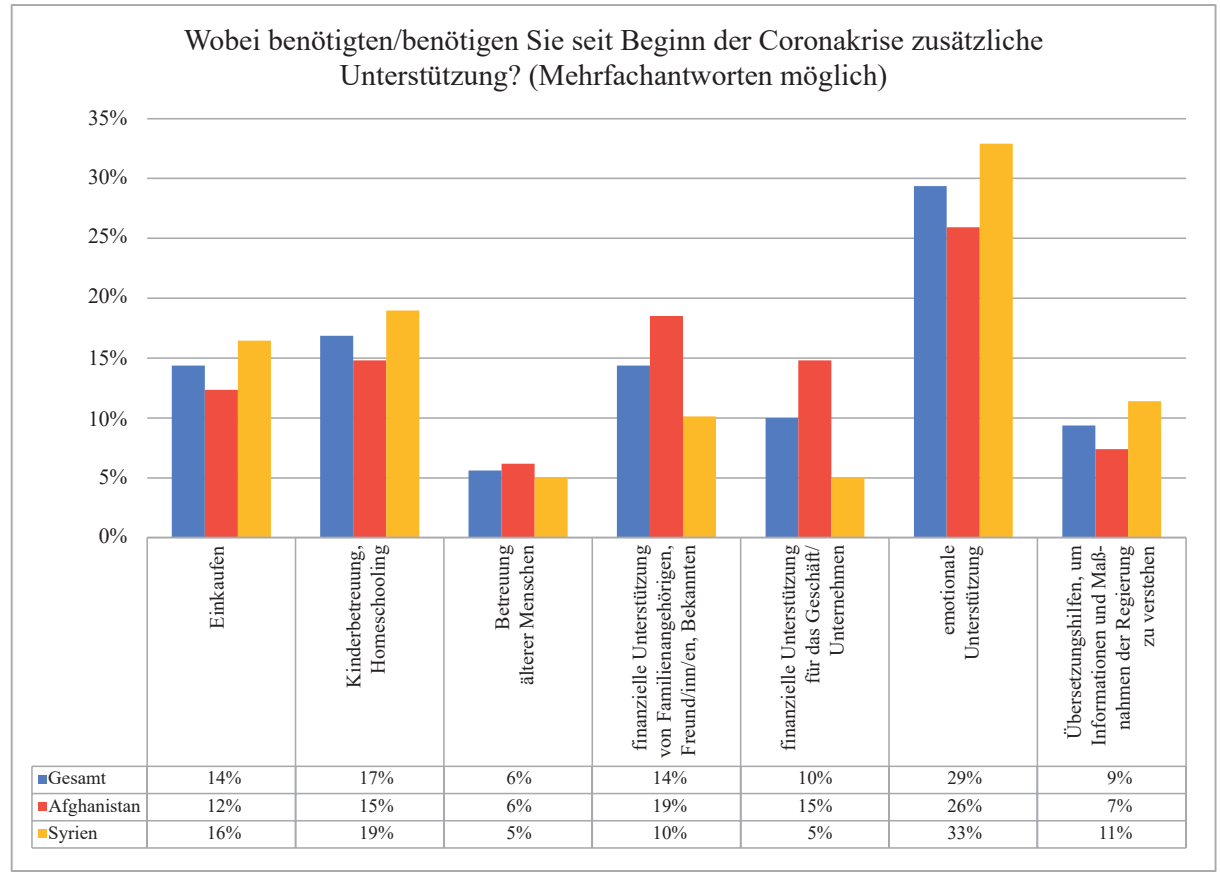

Quelle: eigene Erhebung 2020.

Befragte aus beiden Gruppen benötigten vor allem emotionale Unterstützung (Afghanistan 26\%, Syrien 33\%). An zweiter Stelle lag in der afghanischen Herkunftsgruppe finanzieller Support für Familienangehörige und Freund/inn/e/n (19\%), mit jeweils $15 \%$ folgten Hilfe beim Homeschooling sowie zusätzliche finanzielle Mittel für 
das Unternehmen. Beim Einkaufen bestand für 12\% ein Bedarf an zusätzlicher Unterstützung. Syrische Befragte bedurften vor allem der Hilfe bei der Kinderbetreuung und beim Homeschooling und beim beim Einkaufen (16\%). Support bei Übersetzungen wurde von 11\% nachgefragt und ein Zehntel des Subsamples Syrien holte sich für die finanzielle Unterstützung von Familienangehörigen externe Hilfe.

Die Vielfalt der Kontexte, in denen Unterstützung benötigten wurde, kommt in den qualitativen Interviews zum Ausdruck. Wie mehrfach betont, stellte vor allem das Homeschooling für viele ein völlig ungewohntes Feld dar, in dem umfassende Unterstützung benötigt wurde. Dabei waren Unterschiede zwischen den Communities und Veränderungen im Laufe der Pandemie zu beobachten. Während die Online-Angebote einiger Vereine gut angenommen wurden, stießen die Angebote anderer auf weniger Interesse. Die Online-Angebote konnte Defizite beim Spracherwerb nicht wirklich ausgleichen. Zusätzlich kam noch hinzu, dass vor der Pandemie organisierte Unterstützungsangebote in den Vereinen vermehrt von Ehrenamtlichen getragen wurden. Dabei handelte es sich oft um Pensionist/inn/en, die selbst zu einer Risikogruppe zählten, und Face-to-Face-Unterstützungen während und nach dem ersten Lockdown aussetzen mussten.

Unterstützung und Hilfeleistungen wurden insbesondere nachgefragt, wenn Personen aufgrund des Verweilens im öffentlichen Raum mit Polizeistrafen belegt wurden. Mehrfach wurde von xenophob motivierten Aktionen berichtet, die als willkürlich und sachlich ungerechtfertigt dargestellt wurden. Hier haben die NGOs in zahlreichen Fällen Hilfe geleistet, um Strafen zu beeinspruchen, die dann entweder reduziert oder gänzlich aufgehoben wurden. Zu Beginn des ersten Lockdowns wurden sogar Übertretungen von Bezirksgrenzen mit Strafen belegt und manche berichteten von strukturellem Rassismus und Racial Profiling der Polizei und anderer Organe in den öffentlichen Verkehrsmitteln oder im öffentlichen Raum.

Vereine und Organisationen beschränkten sich nach dem Lockdown darauf, Unterstützungsangebote wieder, allerdings oft nur mehr eingeschränkt, anzubieten. Dabei wurden vor allem Hilfestellungen für bürokratische Prozesse, bei Anträgen und vor allem hinsichtlich Übersetzungen ins Deutsche nachgefragt. Einzelne WhatsAppGruppen haben ebenfalls solche Unterstützungshilfen angeboten. Auch auf individueller Ebene ist von Geflüchteten für Geflüchtete Unterstützung organisiert worden, etwa im Bereich der Versorgung von pflegebedürftigen Personen oder der gegenseitigen finanziellen Hilfe.

Möglichkeiten zum Socialising konnten allerdings aufgrund der Pandemievorschriften von den Vereinen nicht wiederaufgenommen werden.

Während und nach dem ersten Lockdown waren einige Befragte nicht mehr imstande, finanzielle Unterstützungen an ihre Angehörigen im Ausland zu leisten. Aufgrund von Arbeitslosigkeit und des Verlusts eines Nebenjobs konnten diese Unterstützungen nicht mehr aufrechterhalten werden und manche wandten sich an Freund/ inn/e/n und Bekannte aus der Community und auch an die Mitarbeiter/innen der NGOs. 
Wie unterschiedlich die emotionale Unterstützung in Bezug auf die Haushaltsstruktur bzw. die Wohnsituation ausgesehen hat, wird in nachfolgender bivariater Darstellung ersichtlich, wobei Mehrfachantworten möglich waren:

Tab. 11: Bedarf an emotionaler Unterstützung nach Haushaltsstruktur (gesamt)

\begin{tabular}{|l|r|r|r|r|r|r|}
\hline $\begin{array}{c}\text { emotionale } \\
\text { Unterstützung }\end{array}$ & alleine & $\begin{array}{c}\text { bei den } \\
\text { EItern }\end{array}$ & $\begin{array}{c}\text { bei der eige- } \\
\text { nen Familie }\end{array}$ & $\begin{array}{c}\text { Wohnge- } \\
\text { meinschaft }\end{array}$ & $\begin{array}{c}\text { Wohn- } \\
\text { heim }\end{array}$ & $\begin{array}{c}\text { Gesamt- } \\
\text { ergebnis }\end{array}$ \\
\hline zutreffend & $64,00 \%$ & $29,41 \%$ & $20,00 \%$ & $52,94 \%$ & $33,33 \%$ & $35,61 \%$ \\
\hline nicht zutreffend & $36,00 \%$ & $70,59 \%$ & $80,00 \%$ & $47,06 \%$ & $66,67 \%$ & $64,39 \%$ \\
\hline Gesamtergebnis & $\mathbf{1 0 0 , 0 0 \%}$ & $\mathbf{1 0 0 , 0 0 \%}$ & $\mathbf{1 0 0 , 0 0 \%}$ & $\mathbf{1 0 0 , 0 0 \%}$ & $\mathbf{1 0 0 , 0 0 \%}$ & $\mathbf{1 0 0 , 0 0 \%}$ \\
\hline
\end{tabular}

Quelle: eigene Erhebung 2020.

Abb. 7.2: An wen wandten sich die Befragten um Unterstützung? (gesamt und nach Herkunft)

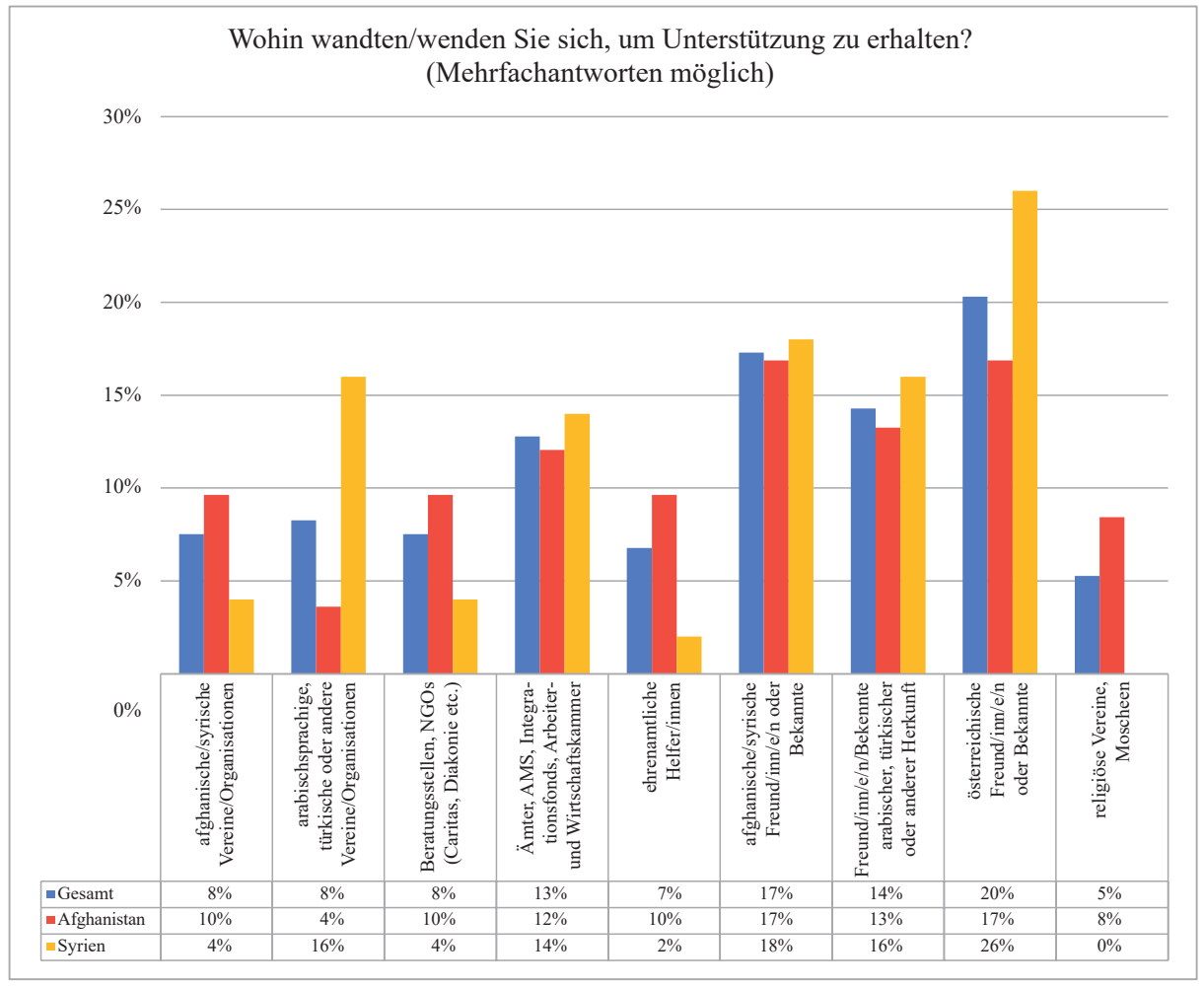

Quelle: eigene Erhebung 2020. 
Zusätzliche emotionale Unterstützung benötigten in erster Linie die in Einpersonenhaushalten lebenden Respondent/inn/en (vgl. Tab. 11). Bei 64\% von ihnen war dies der Fall. Auch wer in Wohngemeinschaften wohnte, bedurfte zu 52,9\% zusätzlichen emotionalen Supports. Geringerer Bedarf bestand in Wohnheimen (ein Drittel) und bei jenen, die mit Eltern im gemeinsamen Haushalt wohnten (29,4\%). Am wenigsten zusätzliche emotionale Hilfe haben jene eingeholt, die mit ihrer Familie zusammenlebten.

Ein interessantes Resultat ist, dass sich ein Fünftel aller Befragten um Unterstützung an österreichische Freund/inn/e/n oder Bekannte gewendet hat, 17\% haben Hilfe bei Freund/inn/e/n aus der eigenen Community erbeten und 13\% haben institutionalisierte Hilfe bei Ämtern, Behörden oder Kammern gesucht (vgl. Abb. 7.2). Etwas weniger wichtig waren mit $8 \%$ NGOs sowie mit gleichen Prozentanteilen afghanische bzw. syrische Vereine oder Organisationen anderer Herkunftsgruppen. Ehrenamtliche Helfer/innen wurden zu 7\% um Hilfe ersucht und am wenigsten wandten sich die Befragten an religiöse Vereine oder Moscheen. Bezüglich des Engagements der Ehrenamtlichen und der geringen Nennung in dieser auch im Zusammenhang mit bereits weiter oben abgehandelten Fragen sind die Erklärungen aus den qualitativen Interviews aufschlussreich. Manche Befragten haben über ihre Freundschaften mit Österreicher/ inne/n berichtet, die sie unter anderem aufgrund deren ehrenamtlichen Tätigkeiten oder Beschäftigungen in NGOs geschlossen haben. Die Übergänge sind hier fließend und nicht einfach in fixen Kategorien darstellbar.

Afghan/inn/en haben zu identen Anteilen von 17\% jeweils österreichische bzw. afghanische Freund/inn/e/n/Bekannte um Hilfe gebeten, dann folgen ihrer Wichtigkeit nach Freund/inn/e/n arabischer bzw. türkischer Herkunft und Behörden mit 12\%. Die Vereine der afghanischen Communities sowie ehrenamtlich tätige Personen wurden von jeweils einem Zehntel der Befragten zwecks Unterstützung angesprochen. Muslimische Vereine bzw. Moscheen waren für $8 \%$ die Anlaufstelle für benötigte Unterstützungen. Religiöse Vereine wurden somit von den afghanischen Respondent/inn/en wesentlich öfter kontaktiert als seitens der syrischen Befragten, von denen kein einzige $/ \mathrm{r}$ einen konfessionellen Verein bzw. Moschee um Unterstützung bat. Mit 4\% waren Organisationen anderer muslimischer Gruppen für die Befragten aus Afghanistan am wenigsten wichtig.

Bei den Syrer/inne/n leisteten in erster Linie österreichische Freund/inn/e/n und Bekannte Hilfe (26\%), danach folgten Freund/inn/e/n aus der Community (18\%), zu je $16 \%$ wurden arabische Freund/inn/e/n bzw. Organisationen um Support ersucht. Mit 14\% hat sich ein höherer Anteil als bei den Afghan/inn/en an Ämter oder Behörden gewandt. $\mathrm{Zu}$ geringen Anteilen wurden syrische Vereine, NGOs und ehrenamtliche Helfer/innen um Hilfe ersucht.

Gegenseitige Unterstützung wurde in Bezug auf das Homeschooling und die Kinderbetreuung geleistet. Einige Respondent/inn/en meinten, dass dies alters-, geschlechts- und communityspezifisch unterschiedlich ausgeprägt war. 
Tab. 12: Unterstützung durch unterschiedliche Personen und Organisationen nach der Aufenthaltsdauer (gesamt) ${ }^{13}$

\begin{tabular}{|c|c|c|c|c|c|c|c|}
\hline & vor 2000 & $\begin{array}{l}2001- \\
2009\end{array}$ & $\begin{array}{l}2010- \\
2014\end{array}$ & 2015 & $\begin{array}{l}2016- \\
2018\end{array}$ & $\begin{array}{l}\text { keine } \\
\text { Angabe }\end{array}$ & $\begin{array}{l}\text { Gesamt- } \\
\text { ergebnis }\end{array}$ \\
\hline \multicolumn{8}{|c|}{ Anzahl von afghanischen/syrischen Vereinen/Organisationen } \\
\hline nicht zutreffend & $87,50 \%$ & $81,25 \%$ & $100,00 \%$ & $95,35 \%$ & $84,21 \%$ & $94,12 \%$ & $92,42 \%$ \\
\hline zutreffend & $12,50 \%$ & $18,75 \%$ & $0,00 \%$ & $4,65 \%$ & $15,79 \%$ & $5,88 \%$ & $7,58 \%$ \\
\hline Gesamtergebnis & $100,00 \%$ & $100,00 \%$ & $100,00 \%$ & $100,00 \%$ & $100,00 \%$ & $100,00 \%$ & $100,00 \%$ \\
\hline \multicolumn{8}{|c|}{ Anzahl von arabischsprachigen, türkischen oder anderen Vereinen/Organisationen } \\
\hline nicht zutreffend & $100,00 \%$ & $100,00 \%$ & $100,00 \%$ & $88,37 \%$ & $84,21 \%$ & $82,35 \%$ & $91,67 \%$ \\
\hline zutreffend & $0,00 \%$ & $0,00 \%$ & $0,00 \%$ & $11,63 \%$ & $15,79 \%$ & $17,65 \%$ & $8,33 \%$ \\
\hline Gesamtergebnis & $100,00 \%$ & $100,00 \%$ & $100,00 \%$ & $100,00 \%$ & $100,00 \%$ & $100,00 \%$ & $100,00 \%$ \\
\hline \multicolumn{8}{|c|}{ Anzahl von Beratungsstellen, NGOs (Caritas, Diakonie etc.) } \\
\hline nicht zutreffend & $100,00 \%$ & $81,25 \%$ & $100,00 \%$ & $88,37 \%$ & $94,74 \%$ & $94,12 \%$ & $92,42 \%$ \\
\hline zutreffend & $0,00 \%$ & $18,75 \%$ & $0,00 \%$ & $11,63 \%$ & $5,26 \%$ & $5,88 \%$ & $7,58 \%$ \\
\hline Gesamtergebnis & $100,00 \%$ & $100,00 \%$ & $100,00 \%$ & $100,00 \%$ & $100,00 \%$ & $100,00 \%$ & $100,00 \%$ \\
\hline \multicolumn{8}{|c|}{$\begin{array}{l}\text { Anzahl von Ämtern, Behörden (z.B. AMS, Integrationsfonds, Arbeiter- bzw. } \\
\text { Wirtschaftskammer) }\end{array}$} \\
\hline nicht zutreffend & $100,00 \%$ & $68,75 \%$ & $86,21 \%$ & $90,70 \%$ & $84,21 \%$ & $94,12 \%$ & $87,12 \%$ \\
\hline zutreffend & $0,00 \%$ & $31,25 \%$ & $13,79 \%$ & $9,30 \%$ & $15,79 \%$ & $5,88 \%$ & $12,88 \%$ \\
\hline Gesamtergebnis & $100,00 \%$ & $100,00 \%$ & $100,00 \%$ & $100,00 \%$ & $100,00 \%$ & $100,00 \%$ & $100,00 \%$ \\
\hline \multicolumn{8}{|c|}{ Anzahl von ehrenamtlichen Helfer/inne/n } \\
\hline nicht zutreffend & $100,00 \%$ & $87,50 \%$ & $86,21 \%$ & $97,67 \%$ & $94,74 \%$ & $94,12 \%$ & $93,18 \%$ \\
\hline zutreffend & $0,00 \%$ & $12,50 \%$ & $13,79 \%$ & $2,33 \%$ & $5,26 \%$ & $5,88 \%$ & $6,82 \%$ \\
\hline Gesamtergebnis & $100,00 \%$ & $100,00 \%$ & $100,00 \%$ & $100,00 \%$ & $100,00 \%$ & $100,00 \%$ & $100,00 \%$ \\
\hline \multicolumn{8}{|c|}{ Anzahl von afghanischen/syrischen Freund/inn/en oder Bekannten } \\
\hline nicht zutreffend & $100,00 \%$ & $56,25 \%$ & $82,76 \%$ & $83,72 \%$ & $84,21 \%$ & $94,12 \%$ & $82,58 \%$ \\
\hline zutreffend & $0,00 \%$ & $43,75 \%$ & $17,24 \%$ & $16,28 \%$ & $15,79 \%$ & $5,88 \%$ & $17,42 \%$ \\
\hline Gesamtergebnis & $100,00 \%$ & $100,00 \%$ & $100,00 \%$ & $100,00 \%$ & $100,00 \%$ & $100,00 \%$ & $100,00 \%$ \\
\hline \multicolumn{8}{|c|}{ Anzahl von Freund/inn/en/Bekannten arabischer, türkischer oder anderer Herkunft } \\
\hline nicht zutreffend & $75,00 \%$ & $75,00 \%$ & $79,31 \%$ & $97,67 \%$ & $89,47 \%$ & $76,47 \%$ & $85,61 \%$ \\
\hline zutreffend & $25,00 \%$ & $25,00 \%$ & $20,69 \%$ & $2,33 \%$ & $10,53 \%$ & $23,53 \%$ & $14,39 \%$ \\
\hline Gesamtergebnis & $100,00 \%$ & $100,00 \%$ & $100,00 \%$ & $100,00 \%$ & $100,00 \%$ & $100,00 \%$ & $100,00 \%$ \\
\hline
\end{tabular}

13 Mehrfachantworten waren möglich. 


\begin{tabular}{|l|r|r|r|c|r|r|r|}
\hline & vor 2000 & $\begin{array}{c}\mathbf{2 0 0 1 -} \\
\mathbf{2 0 0 9}\end{array}$ & $\begin{array}{c}\mathbf{2 0 1 0} \\
\mathbf{2 0 1 4}\end{array}$ & $\mathbf{2 0 1 5}$ & $\begin{array}{c}\mathbf{2 0 1 6} \\
\mathbf{2 0 1 8}\end{array}$ & $\begin{array}{c}\text { keine } \\
\text { Angabe }\end{array}$ & $\begin{array}{c}\text { Gesamt- } \\
\text { ergebnis }\end{array}$ \\
\hline Anzahl von österreichischen Freund/inn/en oder Bekannten \\
\hline nicht zutreffend & $87,50 \%$ & $68,75 \%$ & $79,31 \%$ & $74,42 \%$ & $84,21 \%$ & $82,35 \%$ & $78,03 \%$ \\
\hline zutreffend & $12,50 \%$ & $31,25 \%$ & $20,69 \%$ & $25,58 \%$ & $15,79 \%$ & $17,65 \%$ & $21,97 \%$ \\
\hline Gesamtergebnis & $\mathbf{1 0 0 , 0 0 \%}$ & $\mathbf{1 0 0 , 0 0 \%}$ & $\mathbf{1 0 0 , 0 0 \%}$ & $\mathbf{1 0 0 , 0 0 \%}$ & $\mathbf{1 0 0 , 0 0 \%}$ & $\mathbf{1 0 0 , 0 0 \%}$ & $\mathbf{1 0 0 , 0 0 \%}$ \\
\hline Anzahl von religiösen Vereinen, Moscheen \\
\hline nicht zutreffend & $100,00 \%$ & $75,00 \%$ & $100,00 \%$ & $100,00 \%$ & $89,47 \%$ & $94,12 \%$ & $94,70 \%$ \\
\hline zutreffend & $0,00 \%$ & $25,00 \%$ & $0,00 \%$ & $0,00 \%$ & $10,53 \%$ & $5,88 \%$ & $5,30 \%$ \\
\hline Gesamtergebnis & $\mathbf{1 0 0 , 0 0 \%}$ & $\mathbf{1 0 0 , 0 0 \%}$ & $\mathbf{1 0 0 , 0 0 \%}$ & $\mathbf{1 0 0 , 0 0 \%}$ & $\mathbf{1 0 0 , 0 0 \%}$ & $\mathbf{1 0 0 , 0 0 \%}$ & $\mathbf{1 0 0 , 0 0 \%}$ \\
\hline
\end{tabular}

Quelle: eigene Erhebung 2020.

Die Aufenthaltsdauer hat die Inanspruchnahme bestimmter Institutionen zwecks Unterstützung in der Krise in sehr unterschiedlichem Ausmaß beeinflusst. Organisationen der Communities wurden am wenigsten von den 2015 gekommenen Befragten in Anspruch genommen, am stärksten von den bereits seit 2001-09 (18,8\%) in Österreich lebenden und zu einem etwas geringeren Anteil (15,8\%) von jenen, die in den Jahren 2016-18 ins Land kamen, sowie auch von 12,5\% der vor 2000 migrierten. Geflüchtete der Jahre 2016-18 haben am ehesten Vereine anderer Migrant/inn/engruppen kontaktiert. Mit fast 19\% haben sich am öftesten Personen, die in den Jahren 2001-09 nach Österreich geflüchtet waren, an die österreichischen NGOs um Support gewandt. Dies war auch jene Gruppe, die zu einem hohen Anteil (31,3\%) Ämter und Behörden zwecks Unterstützung kontaktierte. Ehrenamtliche Helfer/innen waren vor allem für die 2010-14 geflüchteten Befragten wichtige Ansprechpersonen. 43,8\% der 2001-09 in Österreich Angekommenen haben in erster Linie ihre Freund/inn/e/n/Bekannten in den Communities um Support gebeten, 31,3\% von ihnen haben aber auch österreichische Freund/inn/e/n/Bekannte kontaktiert, dies taten auch 25,6\% der Personen, die 2015 nach Österreich geflüchtet waren. Letztere scheinen - vielleicht aufgrund des damaligen zivilen Engagements („Refugee Welcome Culture“) - nach wie vor besonders gut mit Österreicher/inne/n vernetzt zu sein. Mit 15,8\% war jedenfalls der Anteil der Geflüchteten der Jahre 2016-18, die Österreicher/innen um Hilfe gebeten hatten, wesentlich geringer. Die bereits länger im Land lebenden Befragten verfügen nicht nur über dichtere Netzwerke zu Einheimischen, ihnen ist auch der Umgang mit Ämtern und Behörden, NGOs und Institutionen vertrauter. 
Tab. 13: Unterstützung durch unterschiedliche Personen und Organisationen nach dem Bildungsniveau (gesamt)

\begin{tabular}{|c|c|c|c|c|c|}
\hline & \begin{tabular}{l}
\multicolumn{1}{c}{ kein } \\
Bildungs- \\
abschluss \\
\end{tabular} & $\begin{array}{l}\text { primärer } \\
\text { Bildungs- } \\
\text { abschluss } \\
\end{array}$ & $\begin{array}{c}\text { sekundärer } \\
\text { Bildungs- } \\
\text { abschluss }\end{array}$ & $\begin{array}{l}\text { tertiärer } \\
\text { Bildungs- } \\
\text { abschluss } \\
\end{array}$ & $\begin{array}{l}\text { Gesamt- } \\
\text { ergebnis }\end{array}$ \\
\hline \multicolumn{6}{|c|}{ Afghanische/syrische Vereine/Organisationen } \\
\hline nicht zutreffend & $80,00 \%$ & $93,75 \%$ & $93,75 \%$ & $91,89 \%$ & $92,42 \%$ \\
\hline zutreffend & $20,00 \%$ & $6,25 \%$ & $6,25 \%$ & $8,11 \%$ & $7,58 \%$ \\
\hline Gesamtergebnis & $100,00 \%$ & $100,00 \%$ & $100,00 \%$ & $100,00 \%$ & $100,00 \%$ \\
\hline \multicolumn{6}{|c|}{ Andere arabischsprachige, türkische oder andere migrantische Vereine/Organisationen } \\
\hline nicht zutreffend & $90,00 \%$ & $93,75 \%$ & $93,75 \%$ & $86,49 \%$ & $91,67 \%$ \\
\hline zutreffend & $10,00 \%$ & $6,25 \%$ & $6,25 \%$ & $13,51 \%$ & $8,33 \%$ \\
\hline Gesamtergebnis & $100,00 \%$ & $100,00 \%$ & $100,00 \%$ & $100,00 \%$ & $100,00 \%$ \\
\hline \multicolumn{6}{|c|}{ Beratungsstellen, NGOs (Caritas, Diakonie etc.) } \\
\hline nicht zutreffend & $100,00 \%$ & $87,50 \%$ & $97,92 \%$ & $86,49 \%$ & $92,42 \%$ \\
\hline zutreffend & $0,00 \%$ & $12,50 \%$ & $2,08 \%$ & $13,51 \%$ & $7,58 \%$ \\
\hline Gesamtergebnis & $100,00 \%$ & $100,00 \%$ & $100,00 \%$ & $100,00 \%$ & $100,00 \%$ \\
\hline \multicolumn{6}{|c|}{ Ämter, Behörden (z.B. AMS, Integrationsfonds, Arbeiter- bzw. Wirtschaftskammer) } \\
\hline nicht zutreffend & $100,00 \%$ & $84,38 \%$ & $87,50 \%$ & $83,78 \%$ & $87,12 \%$ \\
\hline zutreffend & $0,00 \%$ & $15,63 \%$ & $12,50 \%$ & $16,22 \%$ & $12,88 \%$ \\
\hline Gesamtergebnis & $100,00 \%$ & $100,00 \%$ & $100,00 \%$ & $100,00 \%$ & $100,00 \%$ \\
\hline \multicolumn{6}{|c|}{ Ehrenamtliche Helfer/innen } \\
\hline nicht zutreffend & $100,00 \%$ & $100,00 \%$ & $91,67 \%$ & $89,19 \%$ & $93,18 \%$ \\
\hline zutreffend & $0,00 \%$ & $0,00 \%$ & $8,33 \%$ & $10,81 \%$ & $6,82 \%$ \\
\hline Gesamtergebnis & $100,00 \%$ & $100,00 \%$ & $100,00 \%$ & $100,00 \%$ & $100,00 \%$ \\
\hline \multicolumn{6}{|c|}{ Afghanische/syrische Freund/inn/e/n oder Bekannte } \\
\hline nicht zutreffend & $100,00 \%$ & $87,50 \%$ & $77,08 \%$ & $78,38 \%$ & $82,58 \%$ \\
\hline zutreffend & $0,00 \%$ & $12,50 \%$ & $22,92 \%$ & $21,62 \%$ & $17,42 \%$ \\
\hline Gesamtergebnis & $100,00 \%$ & $100,00 \%$ & $100,00 \%$ & $100,00 \%$ & $100,00 \%$ \\
\hline \multicolumn{6}{|c|}{ Freund/inn/e/n/Bekannte arabischer, oder anderer Herkunft } \\
\hline nicht zutreffend & $90,00 \%$ & $87,50 \%$ & $85,42 \%$ & $81,08 \%$ & $85,61 \%$ \\
\hline zutreffend & $10,00 \%$ & $12,50 \%$ & $14,58 \%$ & $18,92 \%$ & $14,39 \%$ \\
\hline Gesamtergebnis & $100,00 \%$ & $100,00 \%$ & $100,00 \%$ & $100,00 \%$ & $100,00 \%$ \\
\hline
\end{tabular}




\begin{tabular}{|l|r|r|r|r|r|}
\hline & $\begin{array}{c}\text { kein } \\
\text { Bildungs- } \\
\text { abschluss }\end{array}$ & $\begin{array}{c}\text { primärer } \\
\text { Bildungs- } \\
\text { abschluss }\end{array}$ & $\begin{array}{c}\text { sekundärer } \\
\text { Bildungs- } \\
\text { abschluss }\end{array}$ & $\begin{array}{c}\text { tertiärer } \\
\text { Bildungs- } \\
\text { abschluss }\end{array}$ & $\begin{array}{r}\text { Gesamt- } \\
\text { ergebnis }\end{array}$ \\
\hline österreichische Freund/inn/e/n oder Bekannte \\
\hline nicht zutreffend & $80,00 \%$ & $81,25 \%$ & $72,92 \%$ & $81,08 \%$ & $78,03 \%$ \\
\hline zutreffend & $20,00 \%$ & $18,75 \%$ & $27,08 \%$ & $18,92 \%$ & $21,97 \%$ \\
\hline Gesamtergebnis & $100,00 \%$ & $100,00 \%$ & $100,00 \%$ & $100,00 \%$ & $100,00 \%$ \\
\hline Religiöse Vereine, Moscheen & & & \\
\hline nicht zutreffend & $100,00 \%$ & $100,00 \%$ & $93,75 \%$ & $94,59 \%$ & $94,70 \%$ \\
\hline zutreffend & $0,00 \%$ & $0,00 \%$ & $6,25 \%$ & $5,41 \%$ & $5,30 \%$ \\
\hline Gesamtergebnis & $100,00 \%$ & $100,00 \%$ & $100,00 \%$ & $100,00 \%$ & $100,00 \%$ \\
\hline
\end{tabular}

Quelle: eigene Erhebung 2020.

In Abhängigkeit vom Bildungsniveau wurden Personen bzw. Institutionen in unterschiedlichem Ausmaß zwecks Unterstützung kontaktiert (vgl. Tab. 13). Organisationen der Communities wurden von einem Fünftel der Respondent/inn/en ohne Bildungsabschluss, aber nur von $8,1 \%$ der Befragten, die tertiäre Abschlüsse aufweisen um Hilfe gebeten. Arabischsprachige oder andere migrantische Vereine waren in erster Linie für Universitätsabsolvent/inn/en (13,5\%) relevant, die Beratungsstellen der NGOs zu einem analogen Prozentsatz sowie zu 12,5\% für die Befragten mit primären Abschlüssen. Hinsichtlich der Kontaktaufnahmen zu Behörden und ehrenamtlichen Helfer/inne/n entfielen auf die Respondent/inn/en mit tertiärer Bildung die höchsten Prozentsätze. Freunde aus den eigenen Communities wurden in erster Linie von Befragten mit sekundären und tertiären Abschlüssen kontaktiert, erheblich seltener von Respondent/inn/en mit Primarschulbildung und gar nicht von Befragten ohne Schulabschlüsse. Freund/ inn/e/n und Bekannte aus Österreich wurden von mehr als $27 \%$ der Interviewees mit Sekundarschulbildung sowie von einem Fünftel der Befragten ohne Schulabschluss involviert, etwas weniger von Uni- und Primarschulabsolvent/inn/en. Religiöse Vereine und Moscheen hatten die geringste Bedeutung. Sie waren für 5,3\% der Befragten, und zwar ausschließlich mit Sekundar- sowie tertiärer Bildung, von Bedeutung.

Die Rahmenbedingungen der Coronakrise haben das Unterstützungsangebot aller NGOs auf vielfältige Weise beeinflusst, was auch in den Expert/inn/eninterviews zum Ausdruck kam (vgl. Abb. 7.3). Die markanteste Veränderung war die Umstellung der Kontaktmöglichkeiten auf Online- bzw. telefonische Kontakte. 28\% gaben an, dass damit auch die Unterstützungsangebote und der Zugang zu selbigen komplizierter wurden. Für 14\% war damit eine Verminderung an Unterstützung verbunden und immerhin ein Zehntel gab an, dass vorher vorhandene Unterstützung sogar gänzlich weggefallen sei. Zusätzliche Angebote haben nur 6\% registriert. 
Abb. 7.3: Veränderungen der Unterstützungsangebote von NGOs während der Coronakrise (gesamt und nach Herkunft)

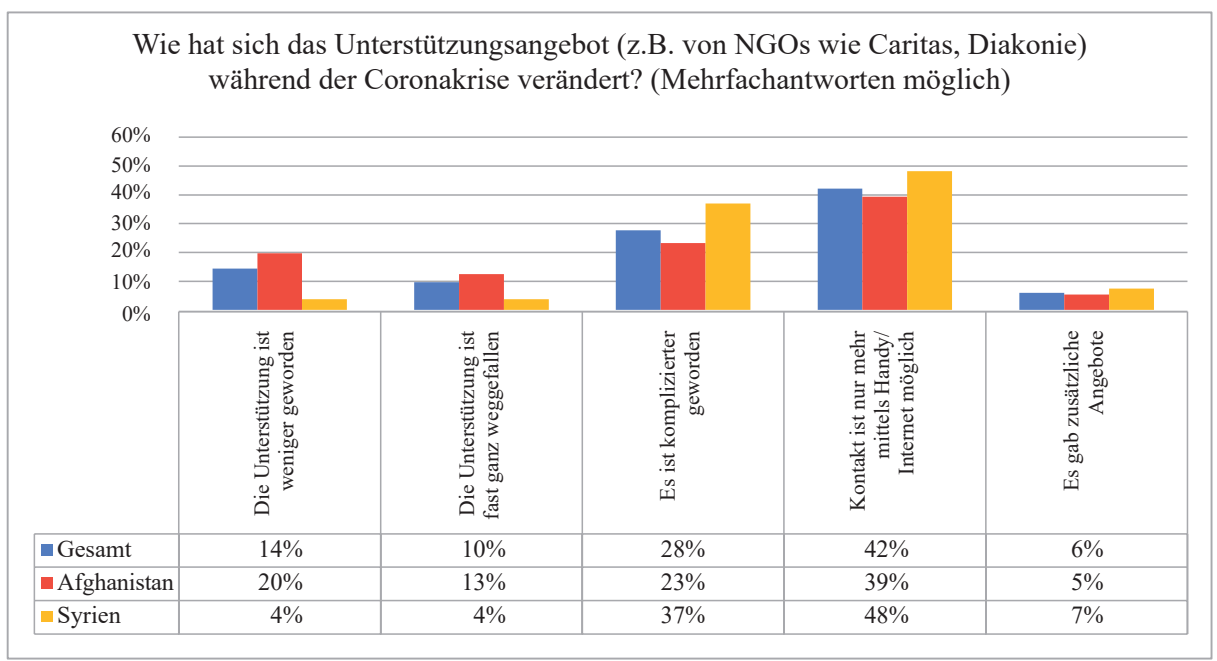

Quelle: eigene Erhebung 2020.

Im Vergleich der Herkunftsgruppen (vgl. Abb. 7.3) wurde von fast der Hälfte (48\%) der syrischen Befragten die Beschränkung der Kontakte zu NGOs auf digitale Medien bzw. Telefon erwähnt, bei den Afghan/inn/en war dieser Anteil mit 39\% beträchtlich geringer. Syrische Respondent/inn/en haben auch viel öfter (37\%) angegeben, dass das Unterstützungsangebot der NGOs komplizierter geworden sei. Unter den Afghan/inn/ en waren nur etwa ein Viertel dieser Ansicht. Dafür haben letztere viel öfter erfahren müssen, dass die Unterstützung reduziert wurde (20\%) oder sogar ganz weggefallen ist (13\%). In der syrischen Subgruppe schätzten dies nur jeweils 4\% auf diese Weise ein. Etwas mehr syrische (7\%) als afghanische (5\%) Befragte erwähnten zusätzliche Angebote seitens der NGOs.

In den Expert/inn/en/interviews wurden die vielfältigen Veränderungen der Unterstützungsangebote beschrieben, wobei die grundlegendsten Änderungen im Wegfall persönlicher Kontakte, der kompletten Umstellung auf Online-Angebote und somit Schwerpunktverlagerungen des gesamten Angebots bestanden. Während die NGOs und deren Mitarbeiter/innen nach unmittelbaren Anfangsschwierigkeiten meist recht gut mit der geänderten Situation umzugehen lernten, kam nur ein Teil der Klient/inn/en der NGOs mit diesen Veränderungen wirklich gut zurecht. Auch seitens der Ehrenamtlichen kam es zu massiven Einschnitten. Viele waren Angehörige der älteren Generation und gehörten somit Risikogruppen an. Sie mussten ihr Engagement häufig völlig ruhendstellen bzw. sich auf Handy bzw. online umorientieren. Für die NGOs gingen damit auch wichtige Personalressourcen verloren, die auch nach Aufhebung des Lockdowns 
nicht mehr im vollen Umfang kompensiert werden konnten. Schwierig dabei war, dass auf Distanz die zuvor umfangreichen Leistungen nicht mehr angeboten werden konnten und daher der Kontakt in Einzelfällen gänzlich verlorenging. Vor allem ältere Klient/inn/en haben es nicht geschafft, diese Online-Angebote in Anspruch zu nehmen. Dazu kamen Einschränkungen im Angebot der Online-Leistungen aufgrund des Datenschutzes, die eigenen Maßnahmen der NGOs zum Schutz der Mitarbeiter/innen und die Verlagerung der Betätigungsfelder für die einzelnen Expert/inn/en. Dadurch ergaben sich bei NGOs neue Abläufe und Umstrukturierungen. Die Kommunikation und vor allem die Übermittlung von notwendigen Unterlagen zur Gewährleistung von Beratung war in manchen Fällen sehr schwierig zu bewerkstelligen. Die dafür nötigen technischen Geräte und Skills (Scanner, PC-Programme, etc.) standen den Klient/inn/en nicht zur Verfügung. Einzelne Vereine und NGOs haben dennoch versucht, über Umwege die Beratungen doch noch zu gewährleisten.

Zusätzlich zu den vorgegebenen Antwortoptionen wurde in Bezug auf das Unterstützungsangebot auch eine offene Kategorie eingeführt. Die in diesem Zusammenhang formulierten Antworten waren sehr unterschiedlich, deckten großteils aber auch die bereits erwähnten Kategorien in den geschlossenen Fragen ab. Weiters ist anzumerken, dass etliche Befragte auch keine Veränderungen bzw. Auswirkungen für ihr eigenes Leben bzw. für ihre Familie feststellten.

Bezüglich der Coping-Strategien erklärte eine Ärztin dass aus medizinischer Sicht die Menschen anfingen bewusst gesünder zu leben, sich bewusster zu ernähren und Sport zu betreiben. Auch wurden die jungen Leute selbständiger in Bezug auf Selbstorganisation.

Die nachfolgenden Darstellungen verweisen auf die Veränderung der Ausrichtungen der Vereine und die Versuche, sich auf die zusätzlichen Bedürfnisse einzustellen.

Die Abb. 7.4 illustriert, in welchen Bereichen das Unterstützungsangebot der Vereine und Organisationen der afghanischen bzw. syrischen Communities während und nach der Coronakrise verändert wurde. Darin ist sowohl die Sicht von außen als auch die Innenperspektive inkludiert, da einige der Respondent/inn/en selbst in Vereinen aktiv waren. Den höchsten Wert nimmt mit 23\% die emotionale Unterstützung ein, gefolgt von finanziellen Hilfestellungen mit $17 \%$ sowie Übersetzungsleistungen aus dem Deutschen. Veränderungen bezüglich der Hilfe zur Bewältigung der sozialen Isolation und Einsamkeit stellten 10\% fest und jeweils $8 \%$ bei juristischer Beratung sowie bezüglich Hilfe bei Besorgungen außer Haus. Support für Ältere und andere Risikogruppen haben $7 \%$ angeführt und für $6 \%$ war die Vermittlung bei sozialen Konflikten relevant. Die geringsten Werte entfallen auf Hilfe beim Homeschooling und die Kinderbetreuung sowie auf Mediation bei familiären Konflikten. 
Abb. 7.4: Veränderungen des Unterstützungsangebots der Vereine der Communities (gesamt und nach Herkunft)

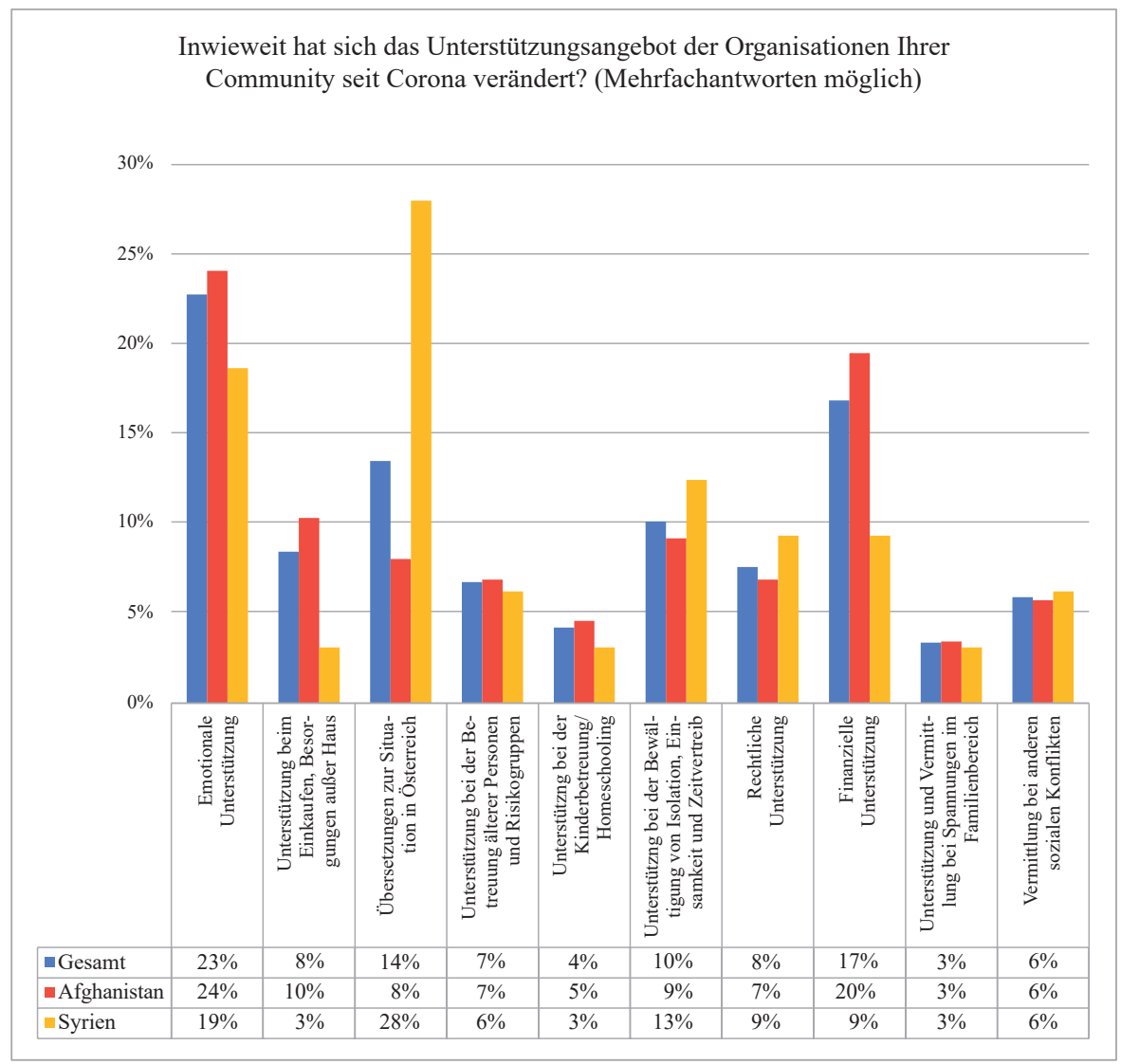

Quelle: eigene Erhebung 2020.

Die Veränderungen sind in Bezug auf die Organisationen der beiden Communities unterschiedlich ausgefallen bzw. wurden seitens der Geflüchteten divergierend rezipiert. Afghanische Befragte haben vor allem emotionale Unterstützung hervorgehoben (24\%), darauf folgte die finanzielle Hilfe (20\%). Die weiteren Kategorien sind sämtlich schwächer besetzt. Unterstützung bei Besorgungen außer Haus mit 10\%, Hilfe bei Bewältigung sozialer Isolation mit 9\%, danach folgen Übersetzungshilfen, Rechtshilfe, Hilfe für Ältere und Risikogruppen sowie Konfliktmediation.

Repondent/inn/en syrischer Herkunft haben mit einem Wert von 28\% vor allem die Veränderung des Unterstützungsangebotes bei Übersetzungen als wesentlich hervorgehoben, gefolgt von emotionaler Hilfe (19\%). Nennenswerte Anteile entfielen sodann 
auf Hilfe zur Bewältigung der Einsamkeit (13\%), auf rechtlichen und finanziellen Support. Bezüglich des Angebots einzelner Communities meinte eine Expertin, dass es mit der Zeit sogar ein Überangebot an Informationsvermittlung gab, welches allerdings teilweise oberflächlich und manchmal irreführend war.

Bezüglich der geleisteten Unterstützungen, die die Befragten angeboten bzw. selbst durchgeführt haben, ist das Bild ebenfalls sehr divergent (vgl. Abb. 7.5).

Abb. 7.5: Unterstützungsleistungen durch die Befragten (gesamt und nach Herkunft)

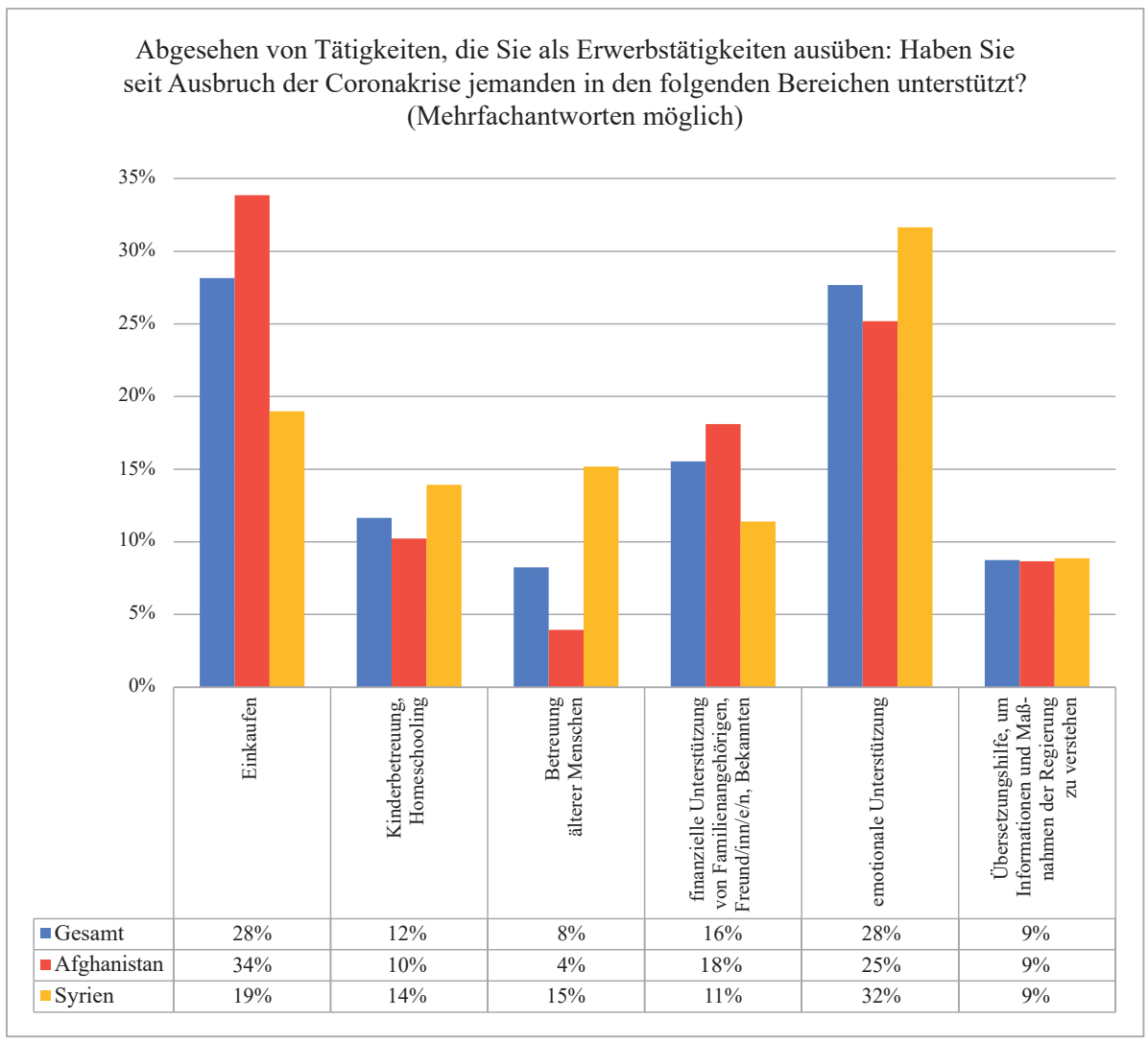

Quelle: eigene Erhebung 2020.

Abb. 7.5 zeigt, welche Personengruppen in welcher Form unterstützt wurden. Am häufigsten wurden mit je $28 \%$ emotionale Unterstützung sowie Hilfe beim Einkaufen angegeben, mit 16\% waren auch Geldleistungen für Familienangehörige und Freund/ inn/e/n eine wichtige Kategorie. 12\% haben im Rahmen von Homeschooling und 
Kinderbetreuung einen Beitrag geleistet, und weniger Personen im Sample haben Übersetzungshilfen sowie die Betreuung von Älteren übernommen.

Auch in Bezug auf die geleisteten Unterstützungen sind Unterschiede nach der Herkunft der Befragten festzustellen. Aus Afghanistan stammende Personen haben am häufigsten beim Einkaufen (34\%) geholfen sowie emotionalen Support geleistet (25\%). Stark repräsentiert ist mit 18\% die Kategorie der Remittances für Familienangehörige und Freund/inn/e/n. Jeweils ein rundes Zehntel hat beim Homeschooling sowie mittels Übersetzungshilfen andere Personen unterstützt.

Bei den syrischen Respondent/inn/en stand die emotionale Unterstützung (32\%) im Vordergrund, dann folgte der Support beim Einkaufen (19\%). Erheblich wichtiger als in der afghanischen Vergleichsgruppe waren für die Syrer/innen die Betreuung älterer Menschen sowie Homeschooling und Kinderbetreuung - beides aus der unterschiedlichen Altersgruppenstruktur sowie den unterschiedlichen Anteilen von Familien mit Kindern erklärbar. Mit 11\% weniger wichtig war die finanzielle Hilfe für Angehörige und Freund/inn/e/n.

Tab. 14: Genderspezifische Unterschiede der Unterstützungsleistungen (gesamt)

\begin{tabular}{|c|c|c|c|}
\hline & männlich & weiblich & Gesamtergebnis \\
\hline \multicolumn{4}{|c|}{ Hilfe beim Einkaufen } \\
\hline nicht zutreffend & $60,81 \%$ & $50,00 \%$ & $56,06 \%$ \\
\hline zutreffend & $39,19 \%$ & $50,00 \%$ & $43,94 \%$ \\
\hline Gesamtergebnis & $100,00 \%$ & $100,00 \%$ & $100,00 \%$ \\
\hline \multicolumn{4}{|c|}{ Unterstützung bei der Kinderbetreuung, Homeschooling } \\
\hline nicht zutreffend & $86,49 \%$ & $75,86 \%$ & $81,82 \%$ \\
\hline zutreffend & $13,51 \%$ & $24,14 \%$ & $18,18 \%$ \\
\hline Gesamtergebnis & $100,00 \%$ & $100,00 \%$ & $100,00 \%$ \\
\hline \multicolumn{4}{|c|}{ Betreuung älterer Menschen } \\
\hline nicht zutreffend & $86,49 \%$ & $87,93 \%$ & $87,12 \%$ \\
\hline zutreffend & $13,51 \%$ & $12,07 \%$ & $12,88 \%$ \\
\hline Gesamtergebnis & $100,00 \%$ & $100,00 \%$ & $100,00 \%$ \\
\hline \multicolumn{4}{|c|}{ Finanzielle Unterstützung von Familienangehörigen, Freund/inn/en, Bekannten } \\
\hline nicht zutreffend & $75,68 \%$ & $75,86 \%$ & $75,76 \%$ \\
\hline zutreffend & $24,32 \%$ & $24,14 \%$ & $24,24 \%$ \\
\hline Gesamtergebnis & $100,00 \%$ & $100,00 \%$ & $100,00 \%$ \\
\hline
\end{tabular}




\begin{tabular}{|l|r|r|r|}
\hline & \multicolumn{1}{|c|}{ männlich } & \multicolumn{1}{c|}{ weiblich } & \multicolumn{1}{|c|}{ Gesamtergebnis } \\
\hline Geleistete emotionale Unterstützung \\
\hline nicht zutreffend & $63,51 \%$ & $48,28 \%$ & $56,82 \%$ \\
\hline zutreffend & $36,49 \%$ & $51,72 \%$ & $43,18 \%$ \\
\hline Gesamtergebnis & $\mathbf{1 0 0 , 0 0 \%}$ & $\mathbf{1 0 0 , 0 0 \%}$ & $\mathbf{1 0 0 , 0 0 \%}$ \\
\hline $\begin{array}{l}\text { Übersetzungshilfe, um Informationen und Maßnahmen der Regierung zu } \\
\text { verstehen }\end{array}$ \\
\hline nicht zutreffend & $83,78 \%$ & $89,66 \%$ & $86,36 \%$ \\
\hline zutreffend & $16,22 \%$ & $10,34 \%$ & $13,64 \%$ \\
\hline Gesamtergebnis & $\mathbf{1 0 0 , 0 0 \%}$ & $\mathbf{1 0 0 , 0 0 \%}$ & $\mathbf{1 0 0 , 0 0 \%}$ \\
\hline
\end{tabular}

Quelle: eigene Erhebung 2020.

In summa haben sich nur wenige genderspezifische Unterschiede herauskristallisiert (vgl. Tab. 14). So haben wesentlich mehr Frauen andere beim Einkaufen (50\% gegenüber $39 \%$ bei Männern) und der Kinderbetreuung (24,1 zu 13,5\%) unterstützt. Besonders ausgeprägt war der Unterschied hinsichtlich der emotionalen Unterstützung, die von 51,7\% der Frauen und nur 36,5\% der Männer erwähnt wird. Übersetzungshilfen haben dafür mehr männliche Befragte geleistet (16,2\% gegenüber 10,3\% der Frauen). Hinsichtlich der Hilfestellungen für Ältere sowie des finanziellen Supports waren die Unterschiede gering.

Tab. 15: Altersspezifische Unterschiede der Unterstützungsleistungen (gesamt)

\begin{tabular}{|c|c|c|c|c|c|c|c|c|}
\hline & $\begin{array}{c}\text { unter } 18 \\
\text { Jahren }\end{array}$ & $\begin{array}{l}\text { 19-29 } \\
\text { Jahre }\end{array}$ & $\begin{array}{l}\text { 30-39 } \\
\text { Jahre }\end{array}$ & $\begin{array}{l}\text { 40-49 } \\
\text { Jahre }\end{array}$ & $\begin{array}{l}\text { 50-59 } \\
\text { Jahre }\end{array}$ & $\begin{array}{l}\text { 60-69 } \\
\text { Jahre }\end{array}$ & $\begin{array}{c}70 \text { oder } \\
\text { älter }\end{array}$ & $\begin{array}{l}\text { Gesamt- } \\
\text { ergebnis }\end{array}$ \\
\hline \multicolumn{9}{|c|}{ Hilfe beim Einkaufen } \\
\hline nicht zutreffend & $100,00 \%$ & $50,82 \%$ & $65,00 \%$ & $59,09 \%$ & $43,75 \%$ & $50,00 \%$ & $100,00 \%$ & $56,06 \%$ \\
\hline zutreffend & $0,00 \%$ & $49,18 \%$ & $35,00 \%$ & $40,91 \%$ & $56,25 \%$ & $50,00 \%$ & $0,00 \%$ & $43,94 \%$ \\
\hline Gesamtergebnis & $100,00 \%$ & $100,00 \%$ & $100,00 \%$ & $100,00 \%$ & $100,00 \%$ & $100,00 \%$ & $100,00 \%$ & $100,00 \%$ \\
\hline \multicolumn{9}{|c|}{ Unterstützung in der Kinderbetreuung, Homeschooling } \\
\hline nicht zutreffend & $100,00 \%$ & $88,52 \%$ & $65,00 \%$ & $72,73 \%$ & $87,50 \%$ & $66,67 \%$ & $100,00 \%$ & $81,82 \%$ \\
\hline zutreffend & $0,00 \%$ & $11,48 \%$ & $35,00 \%$ & $27,27 \%$ & $12,50 \%$ & $33,33 \%$ & $0,00 \%$ & $18,18 \%$ \\
\hline Gesamtergebnis & $100,00 \%$ & $100,00 \%$ & $100,00 \%$ & $100,00 \%$ & $100,00 \%$ & $100,00 \%$ & $100,00 \%$ & $100,00 \%$ \\
\hline \multicolumn{9}{|c|}{ Betreuung älterer Menschen } \\
\hline nicht zutreffend & $100,00 \%$ & $90,16 \%$ & $80,00 \%$ & $86,36 \%$ & $87,50 \%$ & $66,67 \%$ & $100,00 \%$ & $87,12 \%$ \\
\hline zutreffend & $0,00 \%$ & $9,84 \%$ & $20,00 \%$ & $13,64 \%$ & $12,50 \%$ & $33,33 \%$ & $0,00 \%$ & $12,88 \%$ \\
\hline Gesamtergebnis & $100,00 \%$ & $100,00 \%$ & $100,00 \%$ & $100,00 \%$ & $100,00 \%$ & $100,00 \%$ & $100,00 \%$ & $100,00 \%$ \\
\hline
\end{tabular}




\begin{tabular}{|c|c|c|c|c|c|c|c|c|}
\hline & $\begin{array}{c}\text { unter } 18 \\
\text { Jahren }\end{array}$ & $\begin{array}{l}19-29 \\
\text { Jahre }\end{array}$ & $\begin{array}{l}\text { 30-39 } \\
\text { Jahre }\end{array}$ & $\begin{array}{l}40-49 \\
\text { Jahre }\end{array}$ & $\begin{array}{l}50-59 \\
\text { Jahre }\end{array}$ & $\begin{array}{l}60-69 \\
\text { Jahre }\end{array}$ & $\begin{array}{c}70 \text { oder } \\
\text { älter }\end{array}$ & $\begin{array}{l}\text { Gesamt- } \\
\text { ergebnis }\end{array}$ \\
\hline \multicolumn{9}{|c|}{ Finanzielle Unterstützung von Familienangehörigen, Freund/inn/en, Bekannten } \\
\hline nicht zutreffend & $100,00 \%$ & $63,93 \%$ & $75,00 \%$ & $90,91 \%$ & $87,50 \%$ & $83,33 \%$ & $100,00 \%$ & $75,76 \%$ \\
\hline zutreffend & $0,00 \%$ & $36,07 \%$ & $25,00 \%$ & $9,09 \%$ & $12,50 \%$ & $16,67 \%$ & $0,00 \%$ & $24,24 \%$ \\
\hline Gesamtergebnis & $100,00 \%$ & $100,00 \%$ & $100,00 \%$ & $100,00 \%$ & $100,00 \%$ & $100,00 \%$ & $100,00 \%$ & $100,00 \%$ \\
\hline \multicolumn{9}{|c|}{ Emotionale Unterstützung } \\
\hline nicht zutreffend & $66,67 \%$ & $50,82 \%$ & $55,00 \%$ & $72,73 \%$ & $62,50 \%$ & $66,67 \%$ & $25,00 \%$ & $56,82 \%$ \\
\hline zutreffend & $33,33 \%$ & $49,18 \%$ & $45,00 \%$ & $27,27 \%$ & $37,50 \%$ & $33,33 \%$ & $75,00 \%$ & $43,18 \%$ \\
\hline Gesamtergebnis & $100,00 \%$ & $100,00 \%$ & $100,00 \%$ & $100,00 \%$ & $100,00 \%$ & $100,00 \%$ & $100,00 \%$ & $100,00 \%$ \\
\hline \multicolumn{9}{|c|}{ Übersetzungshilfe, um Informationen und Maßnahmen der Regierung zu verstehen } \\
\hline nicht zutreffend & $100,00 \%$ & $78,69 \%$ & $85,00 \%$ & $100,00 \%$ & $93,75 \%$ & $100,00 \%$ & $75,00 \%$ & $86,36 \%$ \\
\hline zutreffend & $0,00 \%$ & $21,31 \%$ & $15,00 \%$ & $0,00 \%$ & $6,25 \%$ & $0,00 \%$ & $25,00 \%$ & $13,64 \%$ \\
\hline Gesamtergebnis & $100,00 \%$ & $100,00 \%$ & $100,00 \%$ & $100,00 \%$ & $100,00 \%$ & $100,00 \%$ & $100,00 \%$ & $100,00 \%$ \\
\hline
\end{tabular}

Quelle: eigene Erhebung 2020.

Auch das Alter der Befragten spielte im Bereich der von diesen geleisteten Unterstützungen für andere eine erhebliche Rolle (vgl. Tab. 15). Hilfe beim Einkaufen wurde vor allem von Älteren ab 50 Jahren sowie den jungen Befragten bis 29 Jahre geleistet. Die Kinderbetreuung übernahmen in erster Linie die 30- bis 39-Jährigen sowie die Großelterngeneration ab 60 Jahren. Letztere war auch zu einem Drittel in die Betreuung älterer Menschen involviert, die jüngeren Altersgruppen erheblich seltener. Finanzielle Unterstützungen wurden in erster Linie von jüngeren Geflüchteten geleistet, nämlich von $36 \%$ der Befragten bis 29 Jahre und 25\% in der Altersgruppe 30 bis 39 Jahre. Emotionale Hilfestellungen für Familienangehörige, Freund/inn/e/n oder Bekannte leisteten mehr als ein Drittel der Altersgruppe bis 29 Jahre und ein Viertel der 30- bis 39-Jährigen. Hinsichtlich der emotionalen Unterstützung zeigt sich einerseits, dass vor allem die Ältesten (ab dem Alter von 70 Jahren) zu 75\% emotionalen Support leisteten, aber auch die jungen Befragten (49\% der bis 29-Jährigen und 45\% der 30bis 39-Jährigen) waren diesbezüglich sehr aktiv, deutlich weniger die mittleren Altersgruppen. Ähnlich ist die Verteilung hinsichtlich der Übersetzungshilfen, die primär in der jüngsten und der ältesten Alterskategorie am häufigsten genannt wurden.

Zwischen den geleisteten Unterstützungen und der Berufsvariable ließen sich keine sehr aussagekräftigen Zusammenhänge feststellen. Hilfestellungen beim Einkaufen, Hilfe für Ältere sowie der emotionale Support für andere Menschen wurde seitens arbeitssuchender Personen, aber auch von Hausfrauen/-männern und Mindestsicherungsempfänger/inne/n häufiger angegeben als von Angestellten. Ein Kausalfaktor dafür wird in deren größeren disponiblen Zeitressourcen liegen. Nicht leicht erklärbar 
ist jedoch, dass auch im Hinblick auf finanzielle Unterstützungen von Angehörigen die Gruppe der Arbeitsuchenden mit 40\% repräsentiert ist, gegenüber nur 29\% der Angestellten. Da für letztere von einem größeren finanziellen Spielraum auszugehen ist, überrascht dieses Resultat. Um hier weitere Schlüsse zu ziehen, wäre es notwendig gewesen ein größeres Sample zu haben. In einem Arbeitsverhältnis stehende Personen, haben am ehesten andere beim Einkaufen unterstützt (37,5\%), wesentlich seltener bei der Betreuung Älterer oder von Kindern, welche ja beträchtlich größere Zeitressourcen erfordert.

Abb. 7.6: Unterstützte Personengruppen (gesamt)

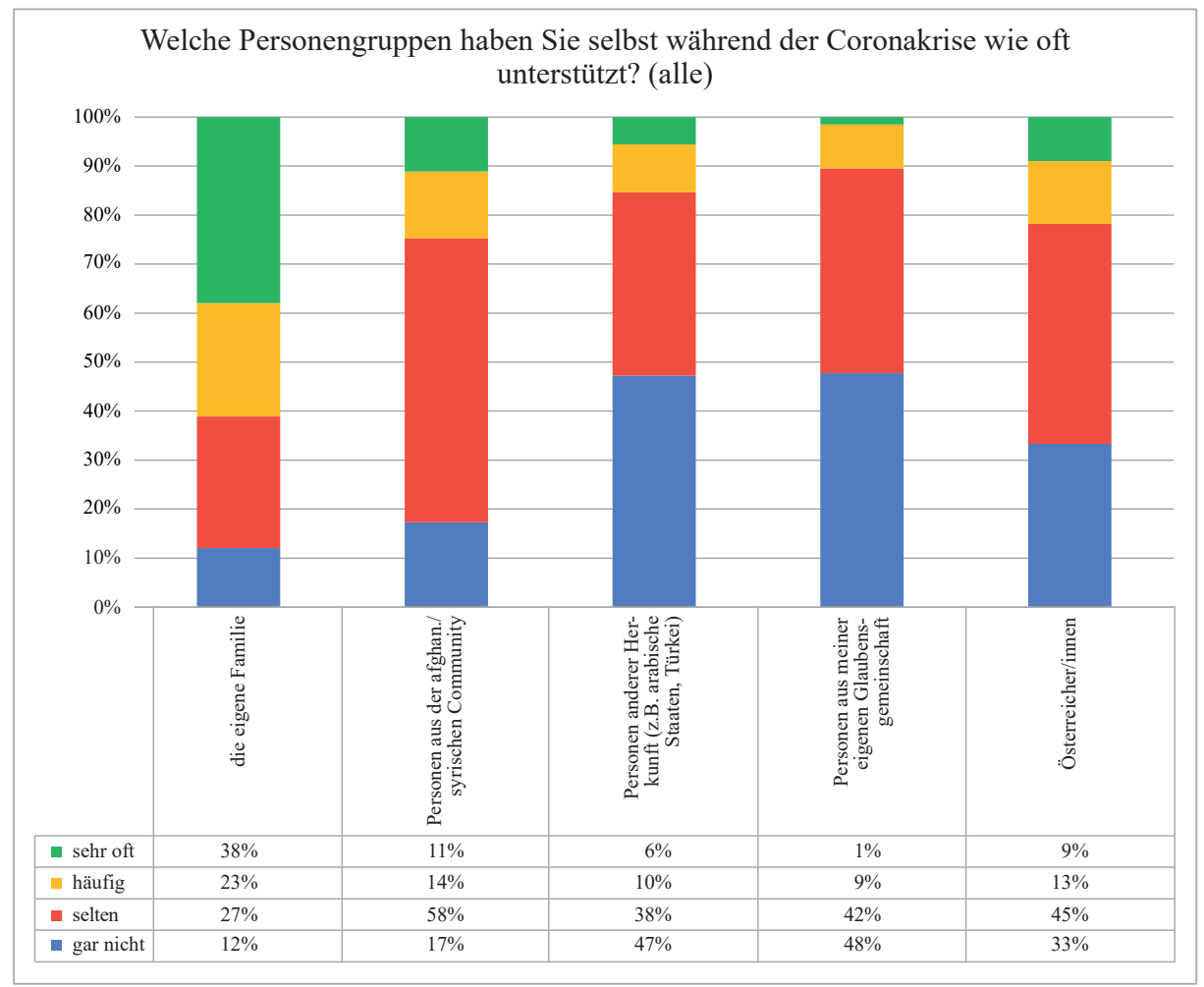

Quelle: eigene Erhebung 2020.

Wenig überraschend war, dass bei der Frage nach den unterstützten Personengruppen besonders die eigene Familie am meisten Unterstützung erfahren hat (vgl. Abb. 7.6). $61 \%$ haben die Familie sehr oft bzw. oft unterstützt. Viel seltener wurden andere Angehörige aus der eigenen Herkunftscommunity unterstützt. Ein Viertel der Befragten tat dies sehr oft bzw. oft, 58\% nur selten. Personen mit anderem Migrationshintergrund 
wurden seltener unterstützt, 47\% erklärten, dass sie dies gar nicht bzw. 38\% dass sie dies selten getan hätten. Noch geringer waren die Unterstützungsleistungen für Angehörige der eigenen Glaubensgemeinschaft, die nur von 10\% der Geflüchteten sehr oft/oft Unterstützung erhielten. Hier ist vielleicht auch zu hinterfragen, wie diese Frage aufgefasst wurde und ob damit auch die eigene Involvierung in eine religiöse Vereinigung verstanden wurde. Öfter wurde Österreicher/inne/n geholfen, nämlich $22 \%$ waren dazu sehr oft/oft bereit und nur ein Drittel gar nicht.

\section{Abb. 7.6.1: Unterstützte Personengruppen (Herkunft Afghanistan)}

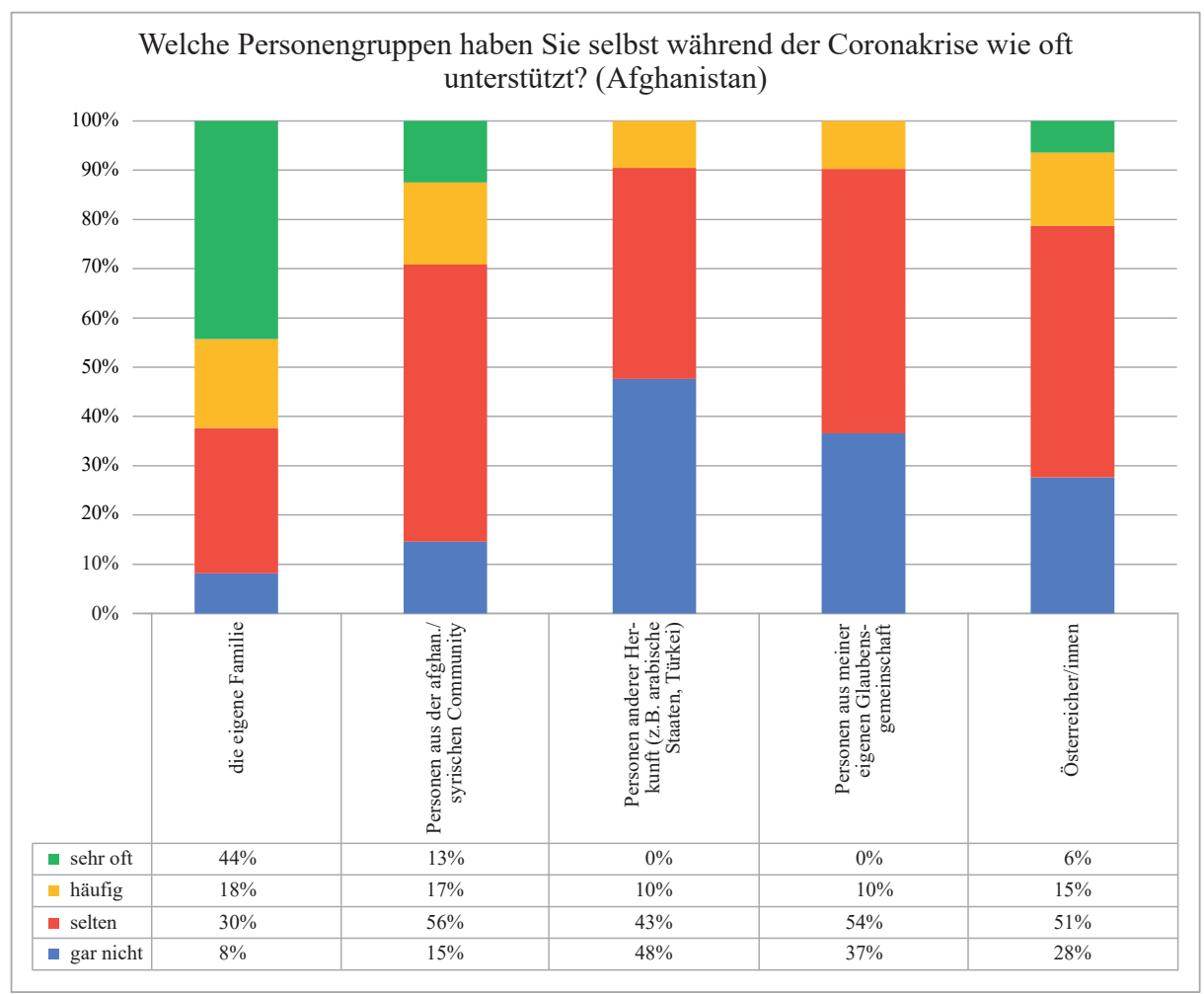

Quelle: eigene Erhebung 2020.

Das Subsample aus Afghanistan hat in erster Linie die eigene Familie unterstützt (vgl. Abb. 7.6.1), nämlich 62\% sehr oft bzw. oft und nur $8 \%$ gar nicht. An zweiter Stelle liegen Freund/inn/e/n aus den afghanischen Communities, die von 30\% oft $/ \mathrm{sehr}$ oft Hilfe erfahren haben, Österreicher/innen wurden von $21 \%$ in derselben Häufigkeit unterstützt. Am seltensten wurde Angehörigen anderer zugewanderter Gruppen bzw. Mitgliedern der eigenen religiösen Konfession geholfen, je 10\% haben dies oft getan. 
In den qualitativen Interviews wurde die Hilfsbereitschaft von Österreicher/inne/n und Freiwilligen besonders betont. In der Pandemie erfolgte eine breite Palette von gegenseitigen Hilfsleistungen und dies vor allem in jenen Netzwerken, die schon zuvor zwischen Familien oder Freund/inn/e/n existierten. Neue Beziehungen, die im Zuge der Pandemie entstanden wären, wurden nicht erwähnt.

Abb. 7.6.2: Unterstützte Personengruppen (Herkunft Syrien)

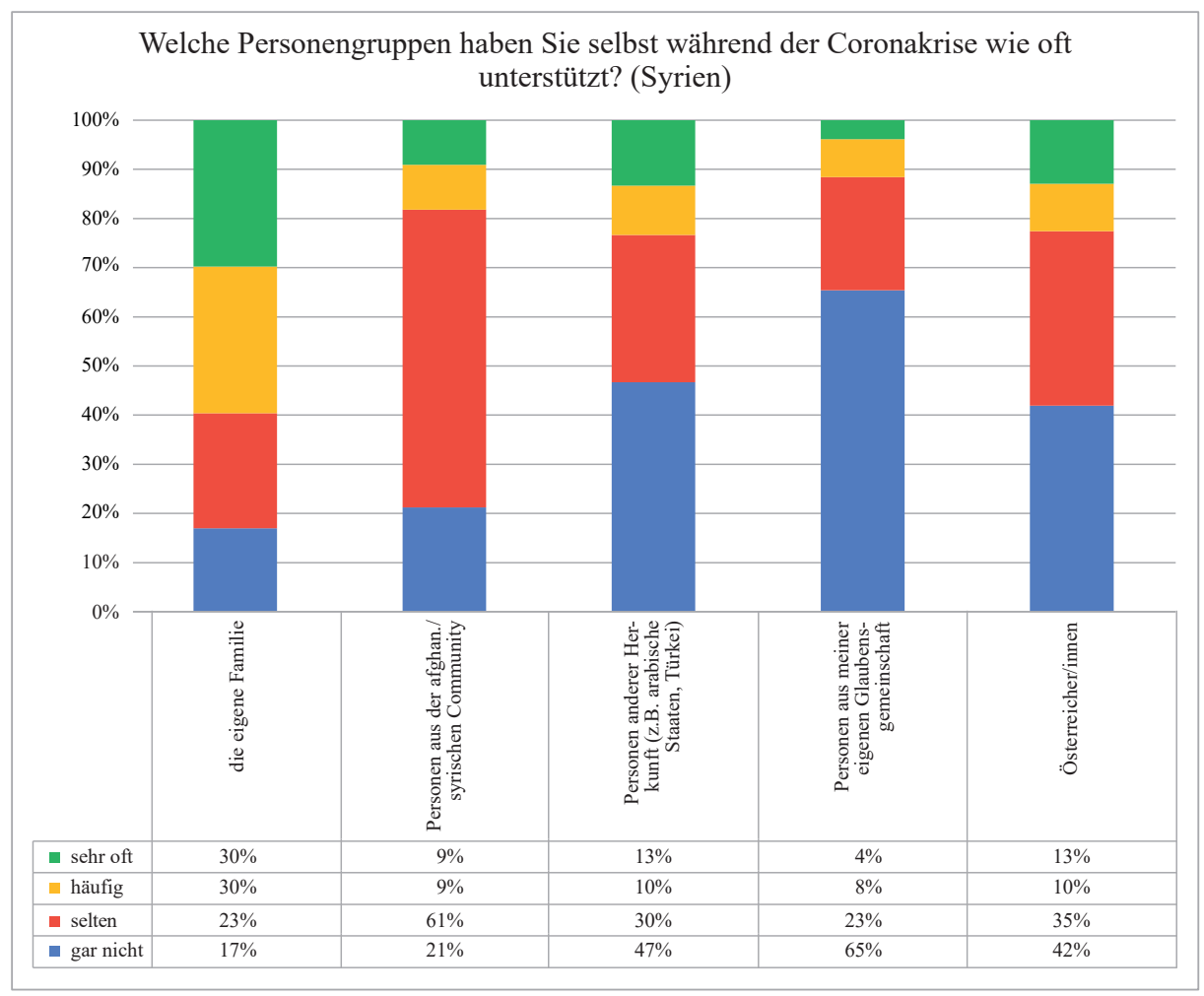

Quelle: eigene Erhebung.

Mehr als die Hälfte der Syrer/innen (vgl. Abb. 7.6.2) haben in der Zeit der Coronakrise ihre Familie unterstützt (dabei ist nicht nach dem Wohnort der Familienmitglieder differenziert worden). Interessant ist auch, dass die Befragten nur zu einem geringen Prozentsatz Mitglieder der eigenen Glaubensgemeinschaft unterstützten (12\% Nennungen sehr oft/oft). Man hat vermehrt Personen anderer Herkunft (arabische Länder, Türkei) oder aus Österreich unterstützt. Daraus lässt sich schließen, dass die eigene Glaubensgemeinschaft kaum als Referenzpunkt für Unterstützungsleistungen 
gilt, aber im Vergleich dazu eher die eigene ethnische oder politische Herkunftsgesellschaft (18\% Nennungen oft/sehr oft und 61\% selten). Mehr als die eigene Glaubensgemeinschaft war man gewillt, Österreicher/innen zu unterstützen. Insgesamt sind dies bemerkenswerte Resultate, die bereits für einen hohen Integrationsgrad sprechen. 


\section{Wirtschaftliche Und finanzielle Probleme}

Abb. 8.1: Auswirkungen der Coronakrise auf die Erwerbstätigkeit und das Einkommen (gesamt und nach Herkunft) ${ }^{14}$

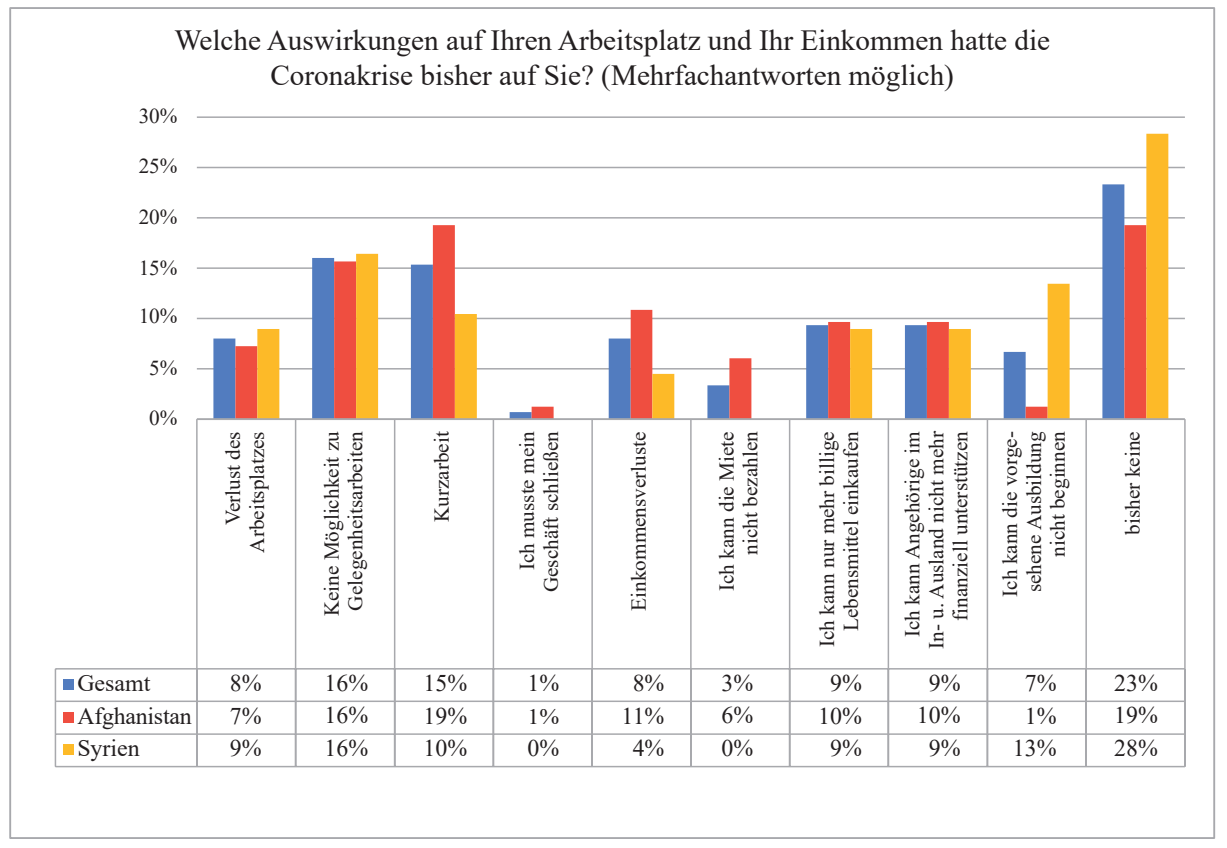

Quelle: eigene Erhebung 2020.

Wie bereits in den vorangegangenen Fragengruppen (vgl. Abb. 4.4 und 5.2) erläutert, waren die Auswirkungen auf den Arbeitsplatz sowie das Einkommen bei vielen Geflüchteten besonders einschneidend und haben hohe Levels an Stress erzeugt. Die Abb. 8.1 illustriert, welche Aspekte im Zusammenhang mit finanziellen Problemen als Folgewirkungen der Coronakrise besonders im Vordergrund standen. Zwar gab nahezu ein Viertel an, dass die Pandemie bis zum Zeitpunkt der Befragung noch keine persönlichen finanziellen Auswirkungen gezeitigt hatte, aber mit 16\%, die den Wegfall von Gelegenheitsarbeiten angaben haben und 15\%, die in Kurzarbeit standen, waren es immerhin fast ein Drittel, welche von diesen beiden Folgewirkungen bereits betroffen waren. Jeweils 9\% konnten Angehörige im Ausland nicht mehr unterstützen oder nur billige Lebensmittel einkaufen, weitere 8\% waren vom Verlust des Arbeitsplatzes betroffen und/oder hatten Einkommensverluste zu bewältigen.

Die beiden Untersuchungsgruppen wurden von den ökonomisch-finanziellen Auswirkungen der Krise in unterschiedlicher Weise in Mitleidenschaft gezogen. $\overline{14}$ Mehrfachantworten waren möglich. 
Bislang keine Auswirkungen gaben 28\% der syrischen, aber nur 19\% der afghanischen Befragten an. Bei Letzteren waren vor allem Kurzarbeit (19\%) und die mangelnde Möglichkeit zu Gelegenheitsarbeiten (16\%) einschneidende Auswirkungen. Es folgten Einkommensverluste (11\%), die Notwendigkeit billige Lebensmittel einzukaufen und der Wegfall von Unterstützungszahlungen an Angehörige (je 10\%).

In der syrischen Herkunftsgruppe spielte der Wegfall von Gelegenheitsarbeiten ebenfalls die größte Rolle, darauf folgten dann aber die Unmöglichkeit eine vorgesehene Ausbildung zu starten (13\%) und zu gleichen Anteilen (je 9\%) der Arbeitsplatzverlust, der Wegfall von Unterstützungen an Angehörige im Herkunftsland und das Einkaufen billiger Waren des täglichen Gebrauchs. Eine viel geringere Rolle als bei den Afghan/ inn/en spielten für die Syrer/innen die Einkommensverluste. Es zeigt sich klar: Die Krise hat vorher bereits bestehende sozioökonomische Ungleichheiten verstärkt und diese sichtbarer werden lassen.

Obwohl medial und seitens der politischen Repräsentant/inn/en vielfach beworben und hervorgehoben, waren die Unterstützungen für selbständige Unternehmer/innen in vielen Fällen nicht in dem Ausmaß garantiert, wie dies immer wieder betont worden war. Der Zugang zu Unterstützungen für kleine Gewerbetreibende erforderte in der Realität die Überwindung zahlreicher formaler und sprachlicher Hürden. Die Vereine haben vor allem Unterstützung in der Informationsbeschaffung geleistet, finanziell konnten sie den Einzelnen keine Hilfe zukommen lassen. Erschwerend kam hinzu, dass kleine Gewerbebetriebe auch meist Familienunternehmen sind, die sonst keine zusätzlichen Einkommen zur Verfügung haben.

Viele unselbständig Beschäftigte, die ihre Arbeit verloren hatten, konnten nur unter großen Mühen die bürokratischen Hürden bewältigen, um Zugang zu Geldleistungen des AMS oder der MA 40 zu finden. Etliche Befragte waren in Wirtschaftsbereichen tätig, die von der Pandemie besonders betroffen waren, nämlich in Gastronomie und Hotellerie. Mit den Optionen auf Kurzarbeit konnten viele ihren Lebensunterhalt kaum bestreiten, auch war die Aussicht auf baldige Wiedereinstellung in diesen Wirtschaftszweigen unrealistisch. Eine Respondentin, die im Handel tätig war, erzählte von ihren Erfahrungen mit dem korrupten Firmenchef, der die Mitarbeiter/innen zur Kurzarbeit anmeldete, allerdings illegal Vollzeitarbeit und sogar Überstunden verlangte.

Es manifestieren sich auch Unterschiede hinsichtlich der Auswirkungen der Krise auf die unterschiedlichen Berufsgruppen, wobei als Bezugsgrößen sowohl die Berufe vor als auch nach der ersten Coronakrise des Frühlings 2020 herangezogen wurden. Vor allem Pensionist/inn/en und Mindestsicherungsbezieher/innen konnten Angehörige im Ausland finanziell nicht mehr unterstützen, Lehrlinge und Angestellte waren davon ebenfalls betroffen, jedoch in einem geringeren Ausmaß.

In erster Linie Hausfrauen und Arbeitssuchende konnten beabsichtigte Aus- und Weiterbildungen nicht starten. Diese beiden Gruppen betraf auch der Wegfall von Gelegenheitsarbeiten am härtesten (jeweils zu 33,3\%). Billigere Grundnahrungsmittel mussten vor allem Lehrlinge einkaufen. Bemerkenswert ist allerdings der relativ hohe 
Anteil jener, für welche die Krise bis zum Befragungszeitpunkt noch keine Auswirkungen gezeitigt hatte. Bei Pensionist/inn/en, Arbeitssuchenden und Bezieher/inne/n der Mindestsicherung waren die diesbezüglichen Anteile am höchsten, da die staatlichen Unterstützungsleistungen stabil blieben, aber auch bei Arbeitnehmer/inne/n im Sample gaben mehr als $41 \%$ an, von ökonomischen Folgewirkungen nicht betroffen gewesen zu sein.

Abb. 8.2: Größte Herausforderungen während und nach der Coronakrise im Vergleich (gesamt und nach Herkunft)

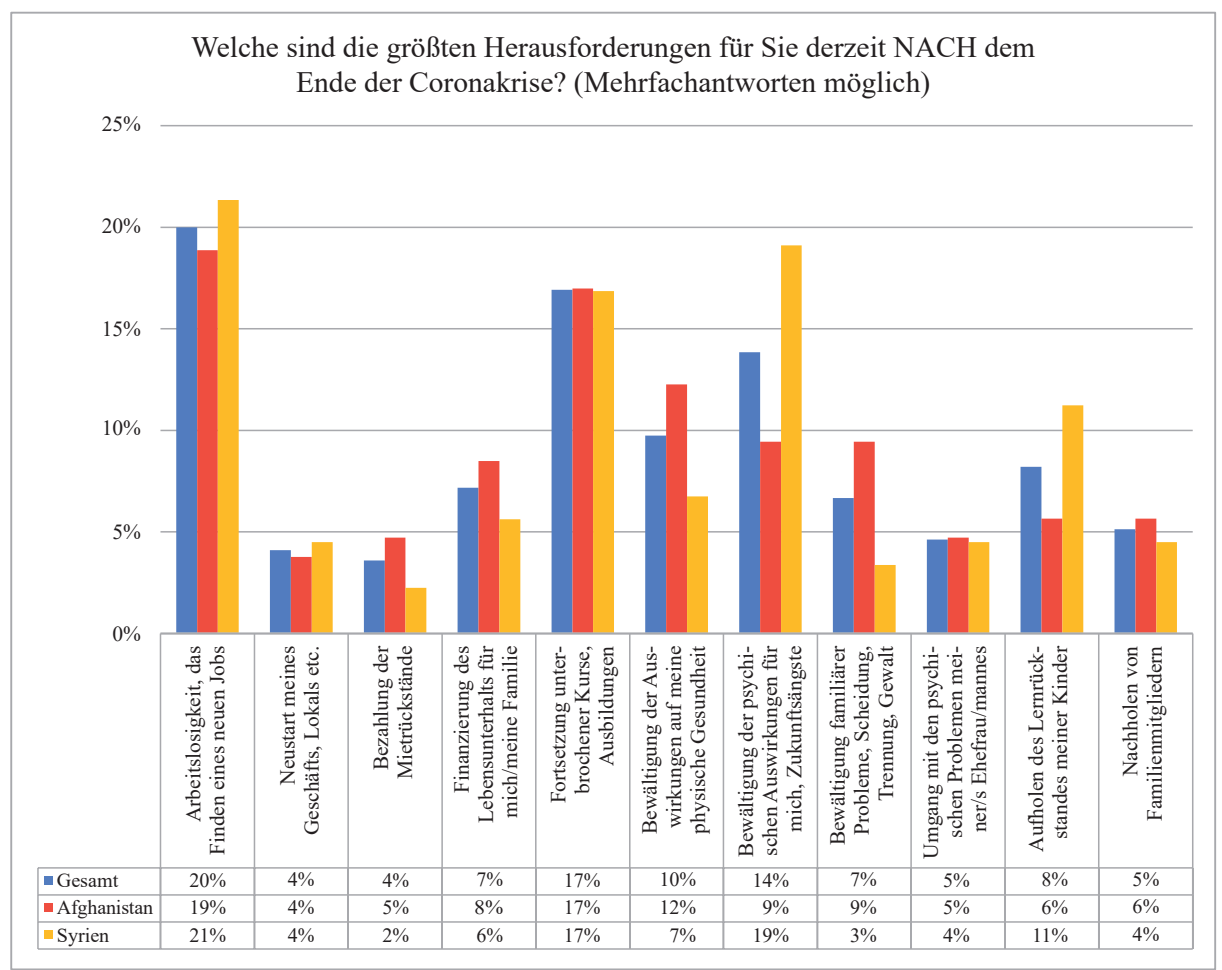

Quelle: eigene Erhebung 2020.

Dem Zeitpunkt der Datenerhebung Rechnung tragend wurde auch nach den größten Herausforderungen während und für die Zeit nach der Coronakrise gefragt (vgl. Abb. 8.2). Damals war noch nicht absehbar, wie lange die Krise de facto andauern würde und ob weitere Lockdowns folgen könnten. Die größten Herausforderungen der Zukunft sahen die Respondent/inn/en in der Arbeitslosigkeit bzw. im Finden einer neuen Beschäftigung (20\%), in der Fortsetzung unterbrochener Ausbildungen (17\%), der Bewältigung der Zukunftsängste (14\%) sowie der Folgewirkungen auf die psychische 
Gesundheit allgemein (10\%). Geringe Bedeutung kam dem Umgang mit psychischen Problemen des/r Ehepartners/in, dem Nachzug von Familienmitgliedern, Mietrückständen sowie dem Geschäftsneustart zu.

Arbeitslosigkeit dominierte die Problemkonstellationen in beiden Herkunftsgruppen, bei den Afghan/inn/en mit 19\% und bei den Syrer/innen mit 21\%. Gleichauf lag mit jeweils $17 \%$ die Fortsetzung unterbrochener Ausbildungen. Ein erheblicher Unterschied zeigte sich in der Kategorie der psychischen Auswirkungen bzw. Zukunftsängste, die bei den syrischen Befragten zu 19\% (Afghan/inn/en nur 9\%) relevant waren. $12 \%$ der Afghan/inn/en und 7\% der Syrer/innen waren um die Bewältigung der psychischen Auswirkungen generell besorgt. Die Aufholung von Lernrückständen bei den Kindern verursachte den syrischen Befragten mit 11\% öfter Sorgen als den Afghan/inn/ en $(6 \%)$, was aber auf den höheren Anteil von Familien mit Kindern zurückzuführen ist. Mietrückstände und Probleme der Finanzierung des Lebensunterhalts der Familie spielten bei 13\% der Afghan/inn/en eine Rolle, bei Syrer/inne/n zu 8\%.

Die größten Herausforderungen zum Befragungszeitpunkt und nach der ersten Coronakrise des Frühlings 2020 lagen bei den Arbeitssuchenden und Hausfrauen/männern in der Suche nach einem neuen Job, bei ersteren aber auch im Neustart eines eigenen Geschäfts sowie in der Finanzierung des Lebensunterhaltes für die Familie. Um eine Fortsetzung unterbrochener Ausbildungen bzw. Kurse sorgten sich Empfänger/ innen der Mindestsicherung, Arbeitssuchende sowie im Haushalt tätige Befragte. Die Bewältigung der Folgewirkungen für die physische Gesundheit bereitete vor allem den Arbeitssuchenden Sorge, hinsichtlich ihrer psychischen Gesundheit waren besonders die Pensionist/inn/en, Hausfrauen/-männer und Empfänger/innen der Mindestsicherung besorgt. Mit 28,6\% war bei Letztgenannten auch der Anteil jener Respondent/inn/en am höchsten, die familiäre Probleme, Trennungen oder Gewalt in der Beziehung zu bewältigen hatten. Psychische Probleme des/der Ehepartner/s/in haben 33,3\% der Pensionist/ inn/en angegeben, das Aufholen der schulischen Lernrückstände der Kinder war am weitesten verbreitet und betraf viele Berufskategorien gleichermaßen, das Nachholen von Familienmitgliedern beunruhigte angesichts der dafür erforderlichen Einkommensnachweise mehr als 14\% der Mindestsicherungsempfänger/innen.

Schließlich bildete eine offene Frage den Abschluss des umfangreichen Fragebogens, in welcher gefragt wurde: „Gibt es abschließend noch etwas, das Sie uns mitteilen wollen? Welche Gedanken und Gefühle haben Sie seit Beginn der CoronaKrise noch beschäftigt?"

Im Rahmen dieser Frage wurde eine große Bandbreite an Statements abgegeben, diese bezogen sich auf Auswirkungen auf die psychische bzw. physische Gesundheit, vielfältige Ängste, die Sorge bezüglich der baldigen Verfügbarkeit eines Impfstoffes. Sehr markant unter den Statements der afghanischen Befragten war jenes, in dem die kriegsbedingte Unsicherheit in Afghanistan mit der durch die Pandemie hervorgerufenen Unsicherheit im öffentlichen Raum verglichen wurde. Beides bedingte, dass die Betroffenen die Seklusion zu Hause suchten. Unter den von syrischen Befragten angege- 
Tab. 16: Antworten auf die offene Abschlussfrage (Auswahl)

\begin{tabular}{|c|c|}
\hline Afghanistan & Syrien \\
\hline 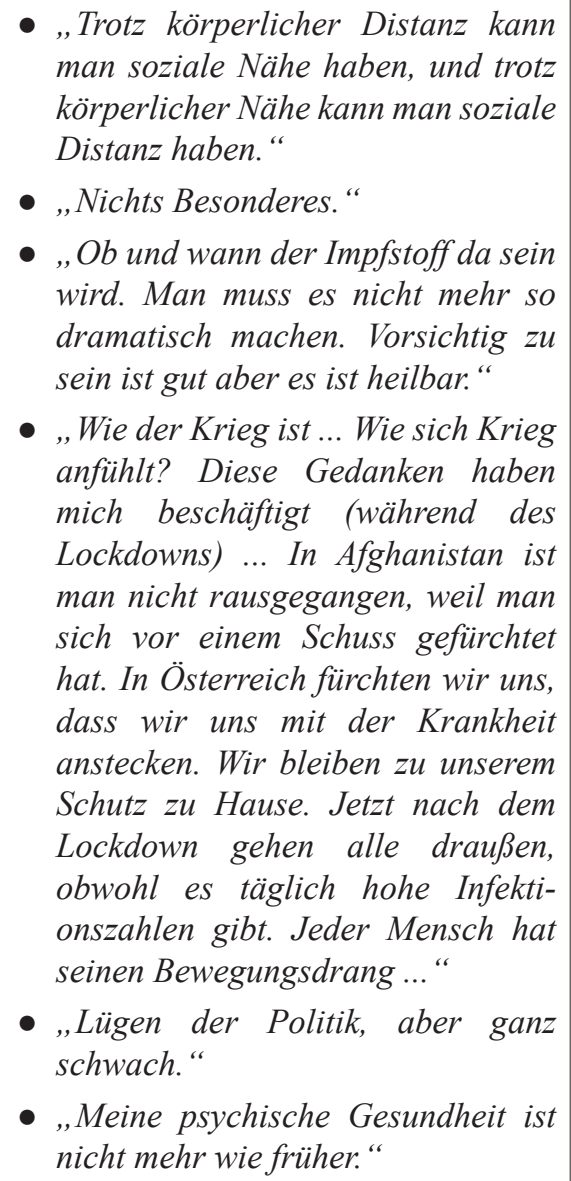 & $\begin{array}{l}\text { - „Ich habe viele Sachen erledigt, } \\
\text { die ich schon seit langem machen } \\
\text { musste, weil ich genug Zeit hatte." } \\
\text { - „Angst vor der Zukunft“ } \\
\text { - „Ich habe Angst und ich frage mich, } \\
\text { ob eine Impfung bald gefunden } \\
\text { wird." } \\
\text { - „Leider, diese Umfrage setzt die } \\
\text { Diskriminierung fort, und wer } \\
\text { diesen Fragebogen gemacht hat, } \\
\text { hat die Diskriminierung übersehen. } \\
\text { Die Isolation von einer Gruppe } \\
\text { aus der gesamten Bevölkerung } \\
\text { und diese Gruppe nicht als Teil der } \\
\text { gesamten Bevölkerung anzusehen. } \\
\text { Ich finde es problematisch, dass } \\
\text { diese Studie die Spaltung der öster- } \\
\text { reichischen Gesellschaft fortsetzt, } \\
\text { obwohl Corona alle betroffen hat." } \\
\text { - Ich habe Angst um meine Familie } \\
\text { und die Menschheit." }\end{array}$ \\
\hline
\end{tabular}

benen Antworten fiel eine auf, welche der Studie durch ihren Fokus auf zwei bestimmte Herkunftscommunities Diskriminierungstendenzen vorwarf. Natürlich hat Corona alle Gruppen in der Gesellschaft betroffen, aber manche Gruppen generell anders und in der Regel heftiger als andere, was auch durch internationale Forschungsergebnisse bestätigt wurde. Viele waren erst vor wenigen Jahren nach Österreich geflohen, manche befanden sich sogar noch in der Unsicherheit des Asylverfahrens. Viele haben ihre erst kürzlich angetretenen Jobs wieder verloren. Die Vulnerabilität als Geflüchtete, ökonomische Marginalisierung, psychische und andere gesundheitliche Probleme akkumulierten sich zu einer komplexen Gemengelage. Diese zu untersuchen und politische Entscheidungsträger auf diese aufmerksam zu machen rechtfertigte den Fokus der vorliegenden Studie. 


\section{RESÜMEE}

Manche der in den unterschiedlichen Untersuchungsbereichen diversifiziert dargestellten Problematiken erscheinen auf den ersten Blick vielleicht repetitiv und in dem einem oder anderen Aspekt vielleicht auch antagonistisch. Bei genauerer Betrachtung ist die Intersektionalität der Problemlagen und der daraus folgenden unterschiedlichen Entwicklungen in der Pandemie sichtbar. Die Komplexität der Auswirkungen lässt sich daher nicht auf einige bestimmte Schwerpunkte hin zusammenfassen oder mit vereinfachten Empfehlungen darstellen. Zweck der Erhebung sollte nicht allein eine Momentaufnahme der Herausforderungen für bestimmte Bevölkerungsgruppen in der Pandemie sein, sondern darauf basierend gut abgestimmte Programme in der Systemintegration zu entwickeln.

Neben den „Hot Topics“ die für die Gesamtbevölkerung galten, wie Physical Distancing und die Überwindung von Social Distancing, Homeschooling, generelle Gesundheitsfragen, sozioökonomische Auswirkungen, war es vor allem die Isolation, mit der die Befragten besonders zu kämpfen hatten. Isolation bezieht sich hier auf die unterschiedlichsten gesellschaftlichen Bereiche, war bereits vor der Pandemie evident und hat sich während derselben weiter verschärft.

Um eine pathologisierende Sicht zu vermeiden, war es von besonderer Bedeutung, die Betroffenen in die Untersuchung möglichst breit einzubinden. Bemerkenswert sind die unterschiedlichsten Initiativen, die von Individuen und Organisationen der Communities ausgingen, um Kommunikationsdefizite, Herausforderungen im Ausbildungsbereich, soziale, rechtliche und gesundheitliche Problem zu bewältigen. Hier haben Soziale Medien sehr dazu beigetragen, dass eine unglaubliche Agency entwickelt wurde. Gerade dieses mannigfaltige zivilgesellschaftliche Engagement, das in der Gesamtgesellschaft kaum sichtbar ist, müsste von der Politik gestärkt werden, da ein ungemeines Potential darin steckt, um die zu erwartenden langfristigen sozialen Auswirkungen der Pandemie nachhaltig meistern zu können.

Die besondere Herausforderung, die zwischen den Diagrammen und Zeilen hervortritt, sind die abhanden gekommenen Zukunftsperspektiven für Betroffene. Viele Befragte haben sich in den vergangenen Jahren neue Perspektiven erarbeiten können, haben Sprachkenntnisse erworben, sind ins Berufsleben eingestiegen etc. Für viele war dies ein neuer Beginn, nachdem sie ihr Leben in der Heimat aufgeben und flüchten mussten. Nun waren und sind sie neuerlich mit Herausforderungen und Zukunftsängsten konfrontiert. Für die Bewältigung der ökonomischen und gesundheitlichen Folgen der Pandemie und neben der Bekämpfung der sozialen Disparitäten gilt es hier, den Menschen wiederum Zukunftsperspektiven zu ermöglichen. 